Sample M1

4700 Reflector Spec \#1 MC=>BC=>NR(2.00)[BP = 1558.0, 18865]

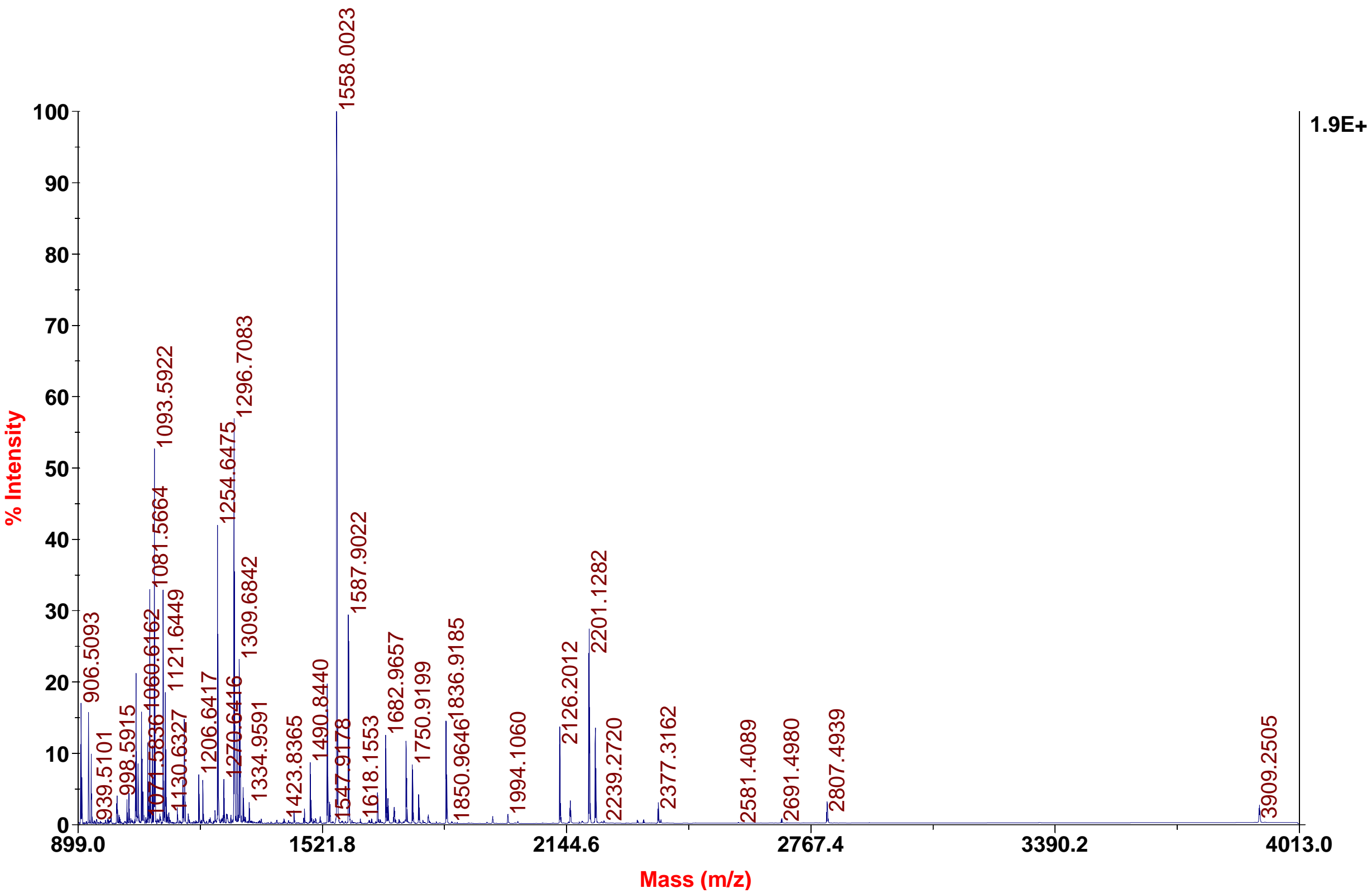




\section{Mascot Score Histogram}

Protein score is $-10 * \log (\mathrm{P})$, where $\mathrm{P}$ is the probability that the observed match is a random event.

Protein scores greater than 62 are significant $(\mathrm{p}<0.05)$.



\section{Concise Protein Summary Report}

Format As Concise Protein Summary .

Significance threshold $p<0.05 \quad$ Max. number of hits AUTO

\section{Re-Search All Search Unmatched}

1. CAR39807 Mass: 53689 Score: 290 Expect: $9.2 e-25$ Matches: 59

MMVMTM NID: - Mus musculus

2. VIME_mOUSE Mass: 53524 Score: 290 Expect: $9.2 e-25$ Matches: 59

V1ment1n.- Mus musculus (Mouse).

Q5FWJ3_MOUSE Mass: 53655 Score: 289 Expect: $1.2 \mathrm{e}-24$ Matches: 59

V1mentin (NOD-derived CD11c +ve dendr1t1c cells cDNA, RIKEN full- length enriched l1brary, clone:F630103E23 product:v1ment1n, CAR69019 Mass: 51533 Score: 282 Expect: 5.8e-24 Matches: 58

MMVIMENT NID: - MUS musculus

Q3TFD9 MOUSE Mass: 53656 Score: 276 Expect: $2.3 e-23$ Matches: 57

17 days embryo heart cDNA, RIKEN full-length enriched library, clone:I920087013 product:v1mentin, full insert sequence.- Mus 


\section{Protein View}

Match to: CAA39807 score: 290 Expect: $9.2 e-25$

\section{MMVMTM NID: - Mus musculus}

Found in search of pmf_A2_129990848800.txt

Nom1nal mass $\left(M_{x}\right)$ : 53689; Calculated pI value: 5.06

NCBI BLAST search of CAA39807 aga1nst $\mathrm{nr}$

unformatted sequence string for past1ng 1nto other applications

Taxonony: Mus musculus

Var1able mod1f1cat1ons: Carban1domethyl (C), Dean1dated (NQ), ox1dation (M)

cleavage by Trypsin: cuts C-term side of KR unless next residue $1 s \mathrm{P}$

Number of mass values searched: 132

Number of mass values matched: 59

sequence coverage: $77 \%$

Matched peptides shown 1n Bold Red

1 MSTRSVSSSS YRRMFGGSGT SSRPSSNRSY VTTSTRTYSL GSALRPSTSR

51 SLYSSSPGGA YVTRSSAVRL RSSVPGVRLL QDSVDFSLAD AINTEFKNTR

101 TNEKVELQEL NDRFANYIDK VRFLEQQNKI LLAELEQLKG QGKSRLGDLY

151 EEEMRELRRO VDOFTNDKAR VEVERDNLAE DIMRLREKLO EEMLOREEAE

201 STLQSFRQDV DNASLARLDL ERKVESLQEE IAFLKKLHDE EIQELQAQIQ

251 EQHVQIDVDV SKPDLTAALR DVRQQYESVA AKNLQEAEEW YKSKFADLSE

301 AANRNNDALR QAKQESNEYR RQVQSLTCEV DALKGTNESL EROMREMEEN

351 FALEAANYQD TIGRLQDEIQ NMKEEMARHL REYQDLLNVK MALDIEIATY

401 RKLLEGEESR ISLPLPTFSS LNLRETNLES LPLVDTHSKR TLLIKTVETR.

451 DGQVINETSQ HHDDLE

Show predicted peptides also

\section{Sort Peptides By $\odot$ Residue Number Increasing Mass Decreasing Mass}

$\begin{array}{rrrr}\text { Start - End } & \text { Observed } & \text { Mr (expt) } & \text { Mr(calc) } \\ 2-12 & 1216.7050 & 1215.6977 & 1215.5844 \\ 5-13 & 1028.5620 & 1027.5547 & 1027.5047 \\ 79-97 & 2126.1985 & 2125.1912 & 2125.0579 \\ 101-113 & 1587.9003 & 1586.8930 & 1586.7900 \\ 105-113 & 1115.6379 & 1114.6306 & 1114.5618 \\ 114-122 & 1125.6663 & 1124.6590 & 1124.5978 \\ 123-129 & 906.5092 & 905.5019 & 905.4607 \\ 123-129 & 908.4391 & 907.4318 & 907.4287 \\ 130-139 & 1169.7773 & 1168.7700 & 1168.7067 \\ 130-143 & 1540.0043 & 1538.9970 & 1538.9032 \\ 146-155 & 1254.6475 & 1253.6402 & 1253.5598 \\ 146-155 & 1270.6410 & 1269.6337 & 1269.5547 \\ 146-158 & 1668.8984 & 1667.8911 & 1667.7824 \\ 160-170 & 1323.7225 & 1322.7152 & 1322.6102 \\ 171-184 & 1688.9368 & 1687.9295 & 1687.8199 \\ 171-184 & 1704.9309 & 1703.9236 & 1703.8148\end{array}$

\begin{tabular}{|c|c|c|}
\hline $\mathrm{pm}$ & Miss & Sequence \\
\hline 93 & 1 & M. STRSVSSSSYR.R \\
\hline 49 & 1 & R. SVSSSSYRR.M \\
\hline 63 & 0 & R. LLQDSVDF SLADAINTEFK. N \\
\hline 65 & 1 & R. TNEKVELQELNDR . F \\
\hline 62 & 0 & K. VELQELNDR . F \\
\hline 54 & 1 & R. FANYIDKVR . F \\
\hline 46 & 0 & R. FLEQQNK. I \\
\hline 3 & 0 & R. FLEQQNK. I 2 Deanidated (NQ) \\
\hline 54 & 0 & K. ILLAELEQLK. G \\
\hline 61 & 1 & K. ILLAELEQLKGQGK. S \\
\hline 64 & 0 & R. LGDLYEEEMR. E \\
\hline 62 & 0 & R. LGDLYEEEMR.E oxidation (M) \\
\hline 65 & 1 & R. LGDLYEEEMRELR. $R$ oxidation (M) \\
\hline 79 & 1 & R. QVDQFTNDKAR.V 2 Deamidated (NQ) \\
\hline 65 & 1 & R. VEVERDNLAEDIMR. L \\
\hline 64 & 1 & R. VEVERDNLAEDIMR. L oxidation (M) \\
\hline
\end{tabular}




\section{M1}

4700 MS/MS Precursor 1093.59 Spec \#1 MC[BP = 175.1, 1637]

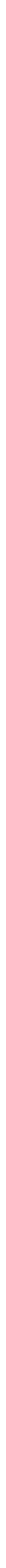




\section{M1}

4700 MS/MS Precursor 1295.75 Spec \#1 MC[BP = 1296.6, 2988]

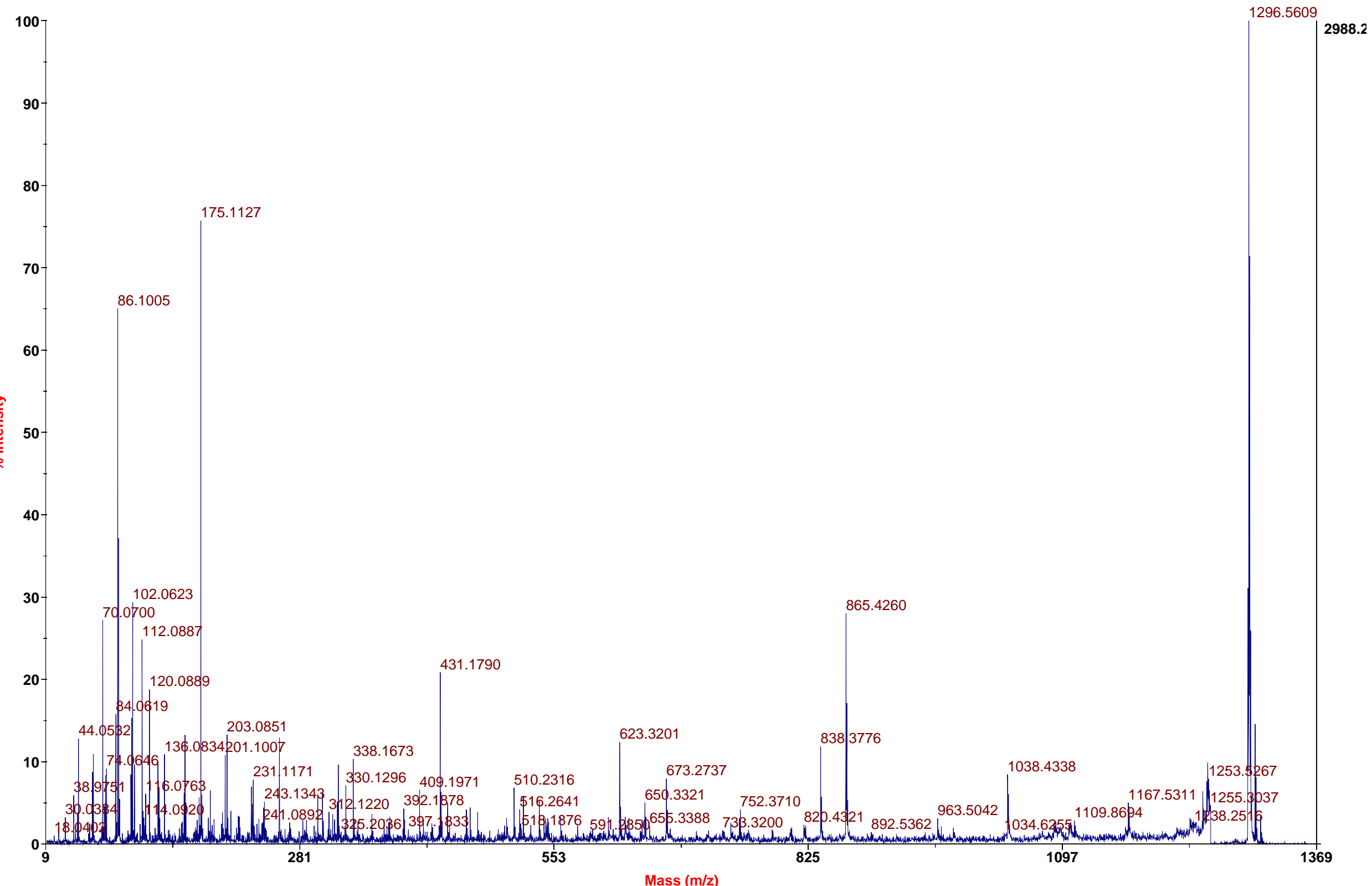




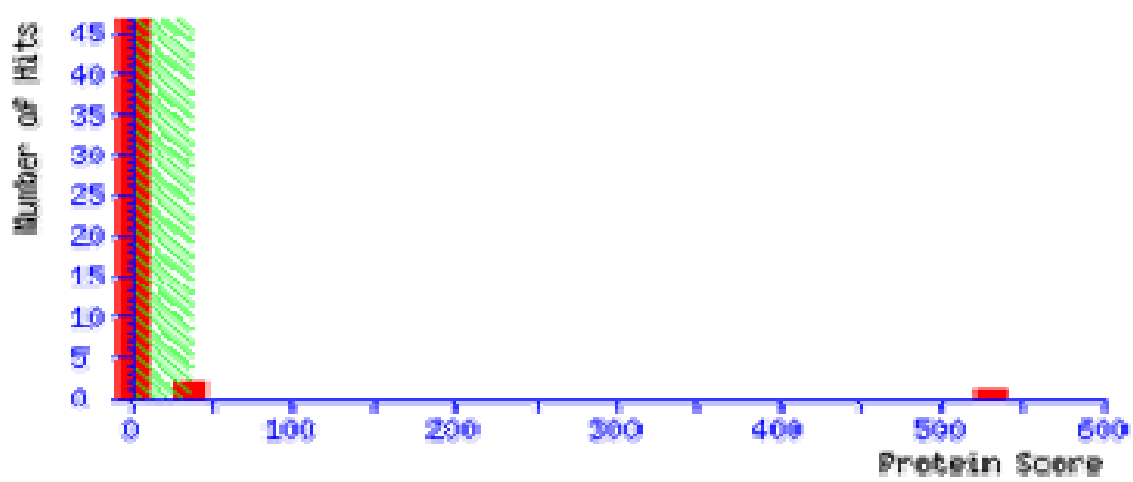

\section{Peptide Summary Report}

\section{Format As Peptide Summary}

Significance threshold $\mathrm{p}<0.05$

Standard scoring $\odot$ MudPIT scoring

Show pop-ups Suppress pop-ups
Max. number of hits AUTO

Ions score or expect cut-off 0

Sort unassigned Decreasing Score $\underline{\text { Help }}$

Show sub-sets 0

Require bold red

\section{Select All}

Select None

Search Selected

\section{$\square$ Error tolerant}

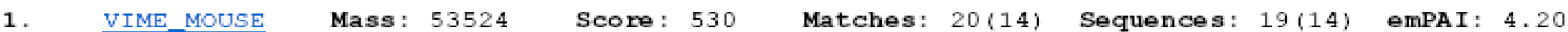
V1ment1n.- Mus nusculus (Mouse).

$\square$ check to 1 nclude this hit $1 \mathrm{n}$ error tolerant search

\begin{tabular}{|c|c|c|c|c|}
\hline \multicolumn{2}{|c|}{ Query } & Observed & Mr (expt) & $\operatorname{Mr}(\mathrm{calc})$ \\
\hline 目 & $\underline{1}$ & 906.5092 & 905.5019 & 905.4607 \\
\hline 回 & $\underline{2}$ & 925.4561 & 924.4488 & 924.3937 \\
\hline 四 & $\underline{3}$ & 1046.6010 & 1045.5937 & 1045.5226 \\
\hline$\sqrt{\nabla}$ & $\underline{4}$ & 1081.5643 & 1080.5570 & 1080.4948 \\
\hline 四 & $\underline{5}$ & 1093.5905 & 1092.5832 & 1092.5200 \\
\hline$\nabla$ & 6 & 1115.6379 & 1114.6306 & 1114.5618 \\
\hline$\nabla$ & $\underline{7}$ & 1169.7773 & 1168.7700 & 1168.7067 \\
\hline$\nabla$ & $\underline{8}$ & 1254.6475 & 1253.6402 & 1253.5598 \\
\hline$\nabla$ & $\underline{9}$ & 1295.7474 & 1294.7401 & 1294.6591 \\
\hline
\end{tabular}

$\begin{array}{lcc}\text { Ppm } & \text { Miss } & \text { Score } \\ 45.6 & 0 & 36 \\ 59.7 & 0 & 33 \\ 68.0 & 0 & 18 \\ 57.6 & 1 & 16 \\ 57.9 & 0 & 58 \\ 61.7 & 0 & 38 \\ 54.2 & 0 & 63 \\ 64.2 & 0 & 52 \\ 62.6 & 0 & 29\end{array}$

$\begin{array}{rcc}\text { Expect } & \text { Rank Unique } \\ 0.072 & 1 & \\ 0.12 & 1 & \mathrm{U} \\ 4.4 & 1 & \mathrm{U} \\ 6 & 1 & \mathrm{U} \\ 0.00051 & 1 & \mathrm{U} \\ 0.043 & 1 & \mathrm{U} \\ 1.9 e-05 & 1 & \mathrm{U} \\ 0.002 & 1 & \mathrm{U} \\ 0.26 & 1 & \mathrm{U}\end{array}$

Peptide

R. FLEQQNK . I

K. QESNEYR . R

K. LQEEMLQR. E

K. QESNEYRR.Q

K . FADLSEAANR . N

K. VELQELNDR . F

K . ILLAELEQLK . G

R. LGDLYEEEMR . E

K. MALDIEIATYR. K 


\section{Protein View}

Match to: VIME_MOUSE Score: 530

Vimentin. - Mus musculus (Mouse).

Found 1n search of ppw_A2_129990854000.txt

Nom1nal mass $\left(M_{x}\right)$ : 53524; Calculated pI value: 5.06

NCBI BLAST search of VIME_MOUSE aga1nst nr

unformatted sequence string for past1ng into other applications

Taxonony: Mus musculus

Var1able modificat1ons: Carban1domethyl (C), Dean1dated (NQ), ox1dation (M) cleavage by Trypsin: cuts c-term side of KR unless next residue $1 s \mathrm{P}$ sequence coverage: 458

Matched peptides shown in Bold Red

1 STRSVSSSSY RRMFGGSGTS SRPSSNRSYV TTSTRTYSLG SALRPSTSRS

51 LYSSSPGGAY VTRSSAVRLR SSVPGVRLLQ DSVDFSLADA INTEFKNTRT 101 NEKVELQELN DRFANYIDKV RFLEQQNKIL LAELEQLKGQ GKSRLGDLYE 151 EEMRELRRQV DQLTNDKARV EVERDNLAED IMRLREKLQE EMLQREEAES 201 TLQSFRQDVD NASLARLDLE RKVESLQEEI AFLKKLHDEE IQELQAQIQE 251 QHVQIDVDVS KPDLTAALRD VRQQYESVAA KNLQEAEEWY KSKFADLSEA

301 ANRNNDALRQ AKQESNEYRR QVQSLTCEVD ALKGTNESLE ROMREMEENF

351 ALEAANYQDT IGRLQDEIQN MKEEMARHLR EYQDLLNVKM ALDIEIATYR

401 KLLEGEESRI SLPLPTFSSL NLRETNLESL PLVDTHSKRT LLIKTVETRD

451 GQVINETSQH HDDLE

Show predicted peptides also

\section{Sort Peptides By Residue Number Increasing Mass Decreasing Mass}

$\begin{array}{rrrr}\text { Start - End } & \text { Observed } & \text { Mr(expt) } & \text { Mr (calc) } \\ 78-96 & 2126.1985 & 2125.1912 & 2125.0579 \\ 100-112 & 1587.9003 & 1586.8930 & 1586.7900 \\ 104-112 & 1115.6379 & 1114.6306 & 1114.5618 \\ 122-128 & 906.5092 & 905.5019 & 905.4607 \\ 129-138 & 1169.7773 & 1168.7700 & 1168.7067 \\ 145-154 & 1254.6475 & 1253.6402 & 1253.5598 \\ 188-195 & 1046.6010 & 1045.5937 & 1045.5226 \\ 222-234 & 1533.9469 & 1532.9396 & 1532.8450 \\ 282-291 & 1309.6841 & 1308.6768 & 1308.5986\end{array}$

$\begin{array}{cclll}\text { ppm } & \text { Miss } & \text { Sequence } \\ 63 & 0 & \text { R. LLQDSVDSLADAINTEFK. N (Ions score 109) } \\ 65 & 1 & \text { R. TNEKVELQELNDR. F (Ions score 58) } \\ 62 & 0 & \text { K. VELQELNDR.F (Ions score 38) } \\ 46 & 0 & \text { R. FLEQQNK. I (IOns score 36) } \\ 54 & 0 & \text { K. ILLAELEQLK. G (Ions score 63) } \\ 64 & 0 & \text { R. LGDLYEEEMR. E (Ions score 52) } \\ 68 & 0 & \text { K. LQEEMLQR. E (Ions score 18) } \\ 62 & 1 & \text { R. KVESLQEEIAFLK. K (Ions score 78) } \\ 60 & 0 & \text { K. NLQEAEEWYK.S (Ions score 46) }\end{array}$


Sample M2

4700 Reflector Spec \#1 MC=>NR(2.00)[BP = 1060.6, 16121]

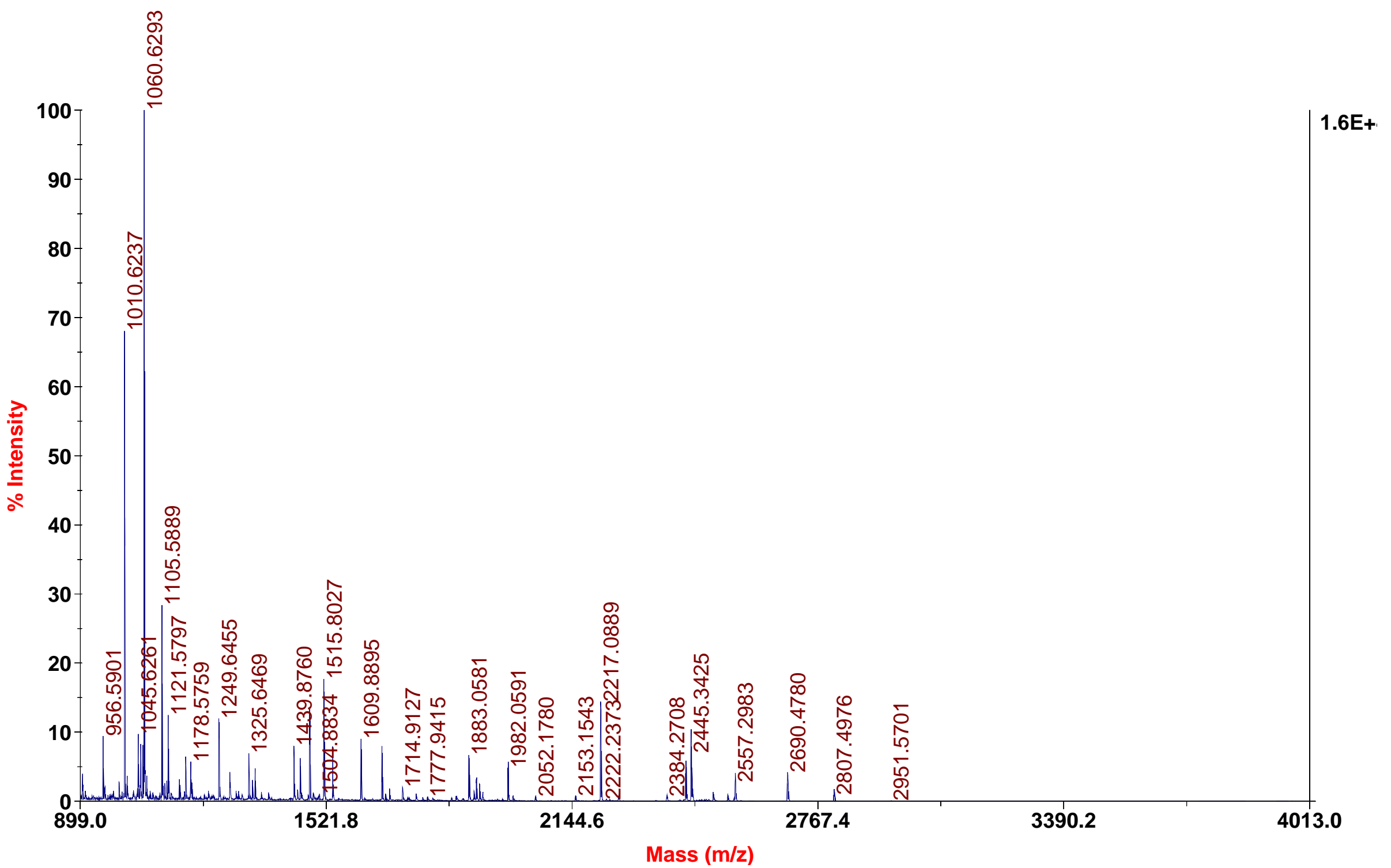




\section{Protein View}

Match to: gec7c7_Mouse score: 132 Expect: 5. Be-o9

2 days neonate thymus thymic cells cDNA, RIKEN full-length enriched library, clone Found $1 \mathrm{n}$ search of pmf_e2_129990848801.txt

Nomlnal mass $\left(\mathrm{M}_{I}\right)$ : 64961 , calculated PI value: 5.49

NCEI ELAST search of gec7c7 MOUSE aga1nst nI

unformatted sequence str1ng for pasting 1nto other applications

Taxonony: Mus musculus

Links to retrleve other entries containing this sequence from NoBI Entrez:

(no taxonomy information for this entry)

Varlable modiflcations: Carbanidonethyl (c), Deanidated (No), oxidation (M) cleavage by Trypein: cuts c-term side of KR unless next residue $1 s$ F

Number of mass values searched: 119

Number of mass values matched: 36

sequence coverage: 59 \%

Matched peptides shown 1n Bold Red

\begin{tabular}{|c|c|c|c|c|}
\hline 1 & 3 & $\mathrm{E}$ & D & FAKTCVADES \\
\hline AANCDRSLHT & LFGDKLCA IP & NLRENYGELA & DCCTKQEPER & NECE 1 \\
\hline FERP & EAEAMCTSEK & El & $Y$ & $\mathrm{E}$ \\
\hline IME 1 & OAEAK & EK & W & \\
\hline ma? & Ls & $\mathrm{DL}$ & 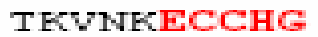 & 6 \\
\hline LAKYMCENO & ATISSF & $\mathrm{H}$ & CLS & $\mathrm{ADL}$ \\
\hline VEDQEVCRNA & YAEARI & TFL & $\mathrm{R}$ & ILE \\
\hline ANFFA & Cxg: & $\mathrm{v}$ & KL & GEYCEQ \\
\hline RYTQRAPQV & STPTI & Grace & $\mathrm{PC}$ & VEDYLS \\
\hline VCLLHEFTP & VSEH & GSLVERRPCE & ETYV & PKEFF \\
\hline Hs & KEK & AELVKHF & एVM & DE \\
\hline & sin & & & \\
\hline
\end{tabular}

Show predicted peptides also

\section{Sort Peptides By}

start - End
$13-25$
$13-33$
$34-43$
$57-65$
$66-73$
$74-85$

Observed

1479.8933

2472.3254

1149.6647

1017.5847

956.5888

1459.6769
Mr (expt) Mr (calc)

1478.9860

2471.3181

1148.6574

1016.5774

955.5815

1458.6696
1478.8497

2471.2042

1148.6077

1016.5291

955.5273

1458.5755
Decreasing Mass

PPm
25
46
43
48
57
65

Miss Sequence

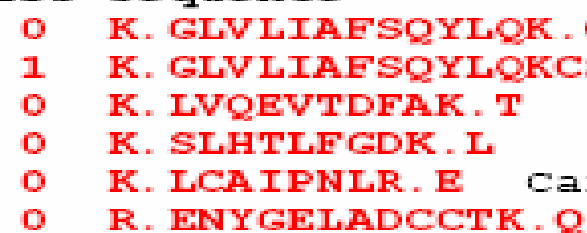


Match to: Q6S9I1_Mouse score: 86 Expect: 0.00021

HMW kininogen-I variant (High molecular weight kininogen I isoform DeltaD5).- Mus muscul Found in search of pmf_B2_129990848801.txt

Nom1nal mass $\left(M_{x}\right): 53172$; calculated pI value: 4.88 NCBI BLAST search of Q6S9I1 MOUSE aga1nst nr unformatted sequence string for pasting into other applications

Taxonorny: Mus musculus

L1nks to retrieve other entries contalning this sequence from NCBI Entrez:

(no taxonomy information for this entry)

(no taxonomy information for this entry)

Var1able mod1f1cations: Carbam1domethyl (C), Deam1dated (NQ), Ox1dation cleavage by Trypsin: cuts c-term side of KR unless next residue $1 s$ P Number of mass values searched: 119

Number of mass values matched: 31

sequence coverage: 518

Matched peptides shown 1n Bold Red

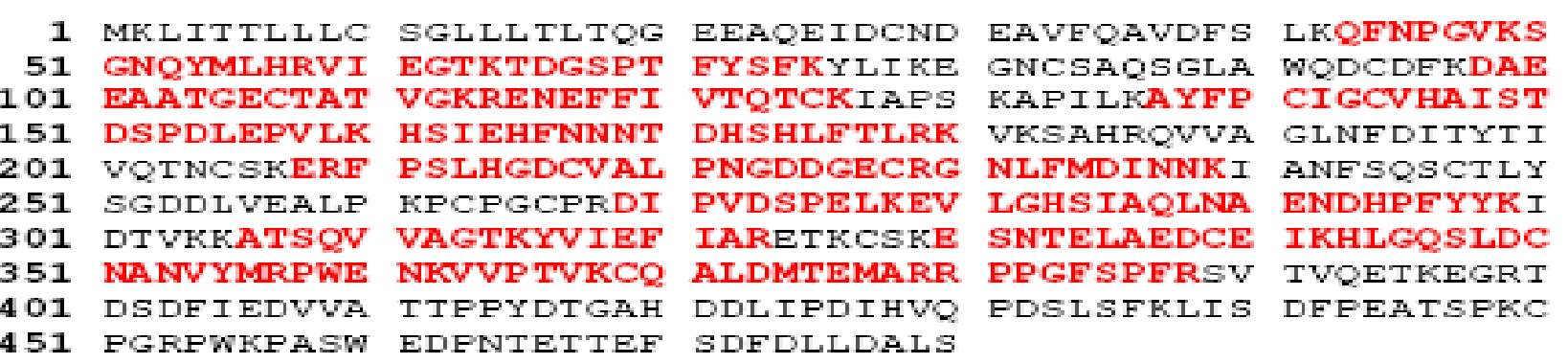

Show predicted peptides also

\section{Sort Peptides By}

Start - End
$43-58$
$43-58$
$50-58$
$50-58$
$50-64$
$65-75$
$98-114$
$114-126$
$115-126$
$115-126$

(o) Residue Number

Obse rved 1895.0566 1896.0194 1105.5889 1121.5780 1749.7764 1249.6459 1765.9055 1671.9216 1459.6769 1515.8025

\section{Mr (expt)} 1894.0493 1895.0121

1104.5816

1120.5707

1748.7691

1248.6386

1764.8982

1670.9143

1458.6696

1514.7952
Increasing Mass

Decreasing Mass

$\begin{array}{rr}\text { Mr (calc) } & \text { ppm } \\ 1893.8679 & 96 \\ 1894.8519 & 85 \\ 1104.5134 & 62 \\ 1120.5084 & 56 \\ 1748.8515 & -47 \\ 1248.5663 & 58 \\ 1764.7948 & 59 \\ 1670.8086 & 63 \\ 1458.6701 & -0 \\ 1514.7075 & 58\end{array}$
11 


\section{M2}

4700 MS/MS Precursor 1010.62 Spec \#1 MC[BP = 86.1, 1894]

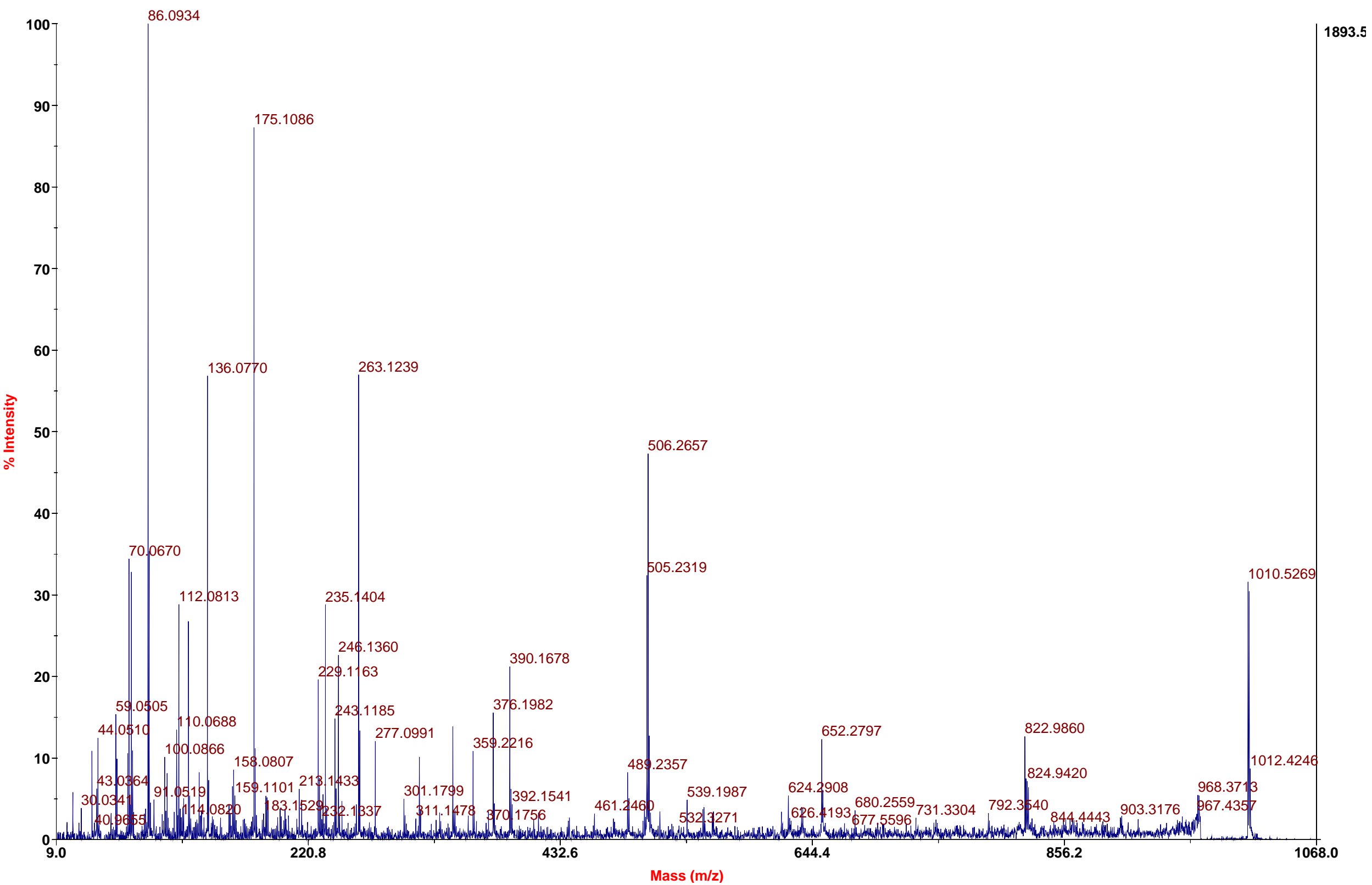




\section{M2}

4700 MS/MS Precursor 1045.63 Spec \#1 MC[BP = 860.9, 3406]






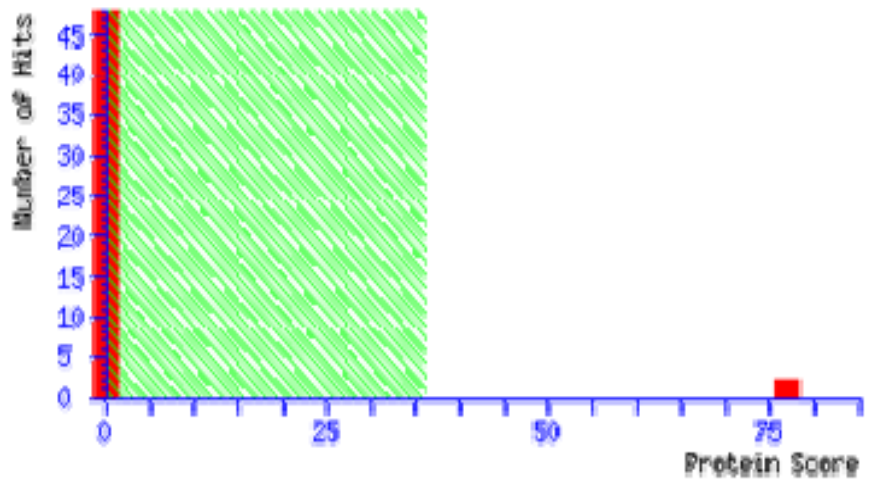

\section{Peptide Summary Report}

\section{Format As Peptide Summary}

Significance threshold $p<0.05$

Standard scoring $\bigcirc$ MudPIT scoring 0 Ions score or expect cut-off 0

Show pop-ups 9 Suppress pop-ups Sort unassigned Decreasing Score $\underline{\text { Help }}$

Show sub-sets 0

Require bold red

\section{$\square$ Error tolerant} O6S9I1 MOUSE Mass: 53172 Score: 77 Matches: 8(1) Sequences: 8(1) emPAI: 0.58 HMW kininogen-I variant (H1gh molecular weight kininogen I 1soform DeltaD5).- Mus musculus (Mouse).

$\square$ check to 1nclude th1s hit in error tolerant search

\begin{tabular}{|c|c|c|c|c|c|c|c|c|c|}
\hline Query & Observed & $\mathrm{Mr}$ (expt) & $\operatorname{Mr}(\mathrm{calc})$ & ppm & Miss & Score & Expect & Rank & Uniq \\
\hline 目 & 1010.6234 & 1009.6161 & 1009.5596 & 55.9 & 0 & 29 & 0.1 & 1 & \\
\hline$\underline{6}$ & 1105.5889 & 1104.5816 & 1104.5134 & 61.7 & 0 & 12 & 21 & 1 & \\
\hline$\underline{8}$ & 1249.6459 & 1248.6386 & 1248.5663 & 57.9 & 0 & 32 & 0.2 & 1 & \\
\hline$\underline{9}$ & 1325.6459 & 1324.6386 & 1324.5574 & 61.4 & 0 & 26 & 0.94 & 1 & \\
\hline$\underline{13}$ & 1515.8025 & 1514.7952 & 1514.7075 & 57.9 & 0 & 32 & 0.2 & 1 & \\
\hline$\underline{25}$ & 1982.0591 & 1981.0518 & 1980.9319 & 60.6 & 1 & 2 & 1. $7 e+02$ & 9 & \\
\hline$\underline{45}$ & 2216.0903 & 2215.0830 & 2214.9423 & 63.5 & 0 & 61 & 0.00026 & 1 & 1 \\
\hline$\underline{60}$ & 2432.2815 & 2431.2742 & 2431.1161 & 65.0 & 0 & 8 & 51 & 1 & \\
\hline
\end{tabular}

\section{Peptide}

K. YVIEFIAR. E

K. SGNQYMLHR.V

K. TDGSPTFYSFK. Y

K. CQALDMTEMAR. R + Carbamidomethyl (C)

R. ENEFFIVTQTCK.I + Carbamidomethyl (C)

U K. VVPTVKCQALDMTEMAR. R + Carbamidomethyl (C); Deamidi

v $\quad \begin{array}{llll}60 & 2432.2815 & 2431.2742 & 2431.116\end{array}$

$65.0 \quad 0$

R. FPSLHGDCVALPNGDDGECR. G + 2 Carbamidomethyl (C)

K. HLGQSLDCNANVYMRPWENK. V + Carbamidomethyl (C) 


\section{Protein View}

Match to: Q6s9I1_Mouse score: 77

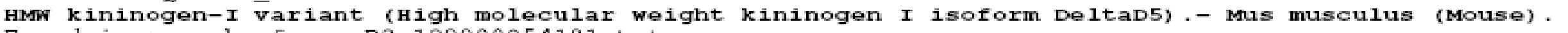

Found in search of ppw_B2_129990854101.txt

Nominal mass $\left(M_{x}\right): 53172$; Calculated pI value: 4.88

NCBI BLAST search of $9659 I 1$ MOUSE against nr

Unformatted sequence string for pasting into other applications

Taxonomy: Mus musculus

Links to retrieve other entries containing this sequence from NCBI Entrez:

(no taxonory information for this entry)

(no taxonomy information for this entry)

Variable modifications: Carbamidonethyl (C), Deamidated (NQ), Oxidation

sequence Coverage: 20 s

Matched peptides shown in Bold Red

1 MKLITTLLLC SGLLLTLTQG EEAQEIDCND EAVEQAVDFS LKQFNPGVKS

51 GNQYMLHRVI EGTKTDGSPT FYSFKYLIKE GNCSAQSGLA WQDCDFKDAE

101 EAATGECTAT VGKRENEFFI VTQTCKIAPS KAPILKAYFP CIGCVHAIST

151 DSPDLEPVLK HSIEHENNNT DHSHLETLRK VKSAHRQVVA GLNEDITYTI

201 VQTNCSKERF PSLHGDCVAL PNGDDGECRG NLFMDINNKI ANESQSCTLY

251 SGDDLVEALP KRCPGCPRDI PVDSPELKEV LGHSIAQLNA ENDHPFYYKI

301 DTVKKATSQV VAGTKYVIEF IARETKCSKE SNTELAEDCE IKHLGQSLDC

351 NANVYMRPWE NKVVPTVKCQ ALDMTEMARR PPGESPFRSV TVOETKEGRT

401 DSDFIEDVVA TTPPYDTGAH DDLIPDIHVQ PDSLSEKLIS DFPEATSPKC

451 PGRPWRPASW EDPNTETTEF SDEDLLDALS

Show predicted peptides also

\section{Sort Peptides By}

$\begin{aligned} \text { start } & - \text { End } \\ 50 & -58 \\ 65 & -75 \\ 115 & -126 \\ 210 & -229 \\ 316 & -323 \\ 343 & -362 \\ 363 & -379 \\ 369 & -379\end{aligned}$

- Residue Number

$\begin{array}{rcr}\text { observed } & \text { Mr(expt) } & \text { Mr (calc) } \\ 1105.5889 & 1104.5816 & 1104.5134 \\ 1249.6459 & 1248.6386 & 1248.5663 \\ 1515.8025 & 1514.7952 & 1514.7075 \\ 2216.0903 & 2215.0830 & 2214.9423 \\ 1010.6234 & 1009.6161 & 1009.5596 \\ 2432.2815 & 2431.2742 & 2431.1161 \\ 1982.0591 & 1981.0518 & 1980.9319 \\ 1325.6459 & 1324.6386 & 1324.5574\end{array}$

Decreasing Mass

\section{ppm Miss sequence}

62 O K. SGNQYMLHR.V (IONS score 12)

58 K. TDGSPTFYSFK. Y (IONS score 32 )

58 R. ENEFFIVTQTCK. I Carbanidomethyl

64 R. FPSLHGDCVALPNGDDGECR.G 2 Carbar.

56

65

61

61
R. FPSLHGDCVALPNGDDGECR.G 2 Carbar.
K. YVIEFIAR.E (IOIS score 29 )
K. HLGQSLDCNANVYMRPWENK. V Carbamid

K. VVPTVKCOALDMTEMAR, R Carbamidome K. CQALDMTEMAR. R Carbanidomethyl

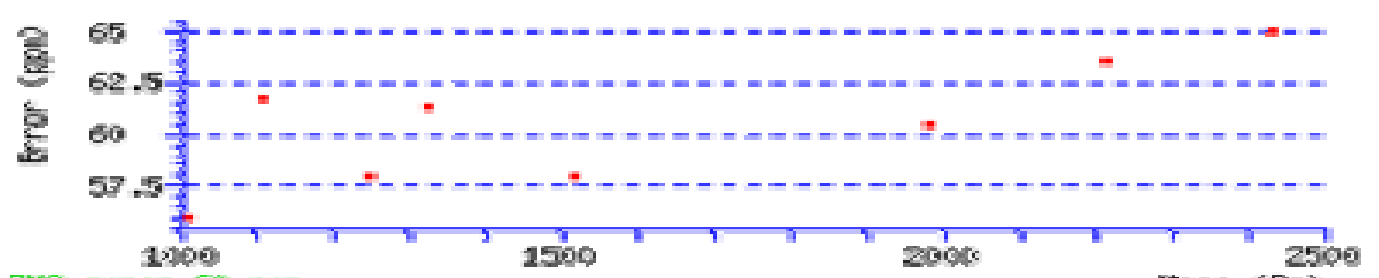

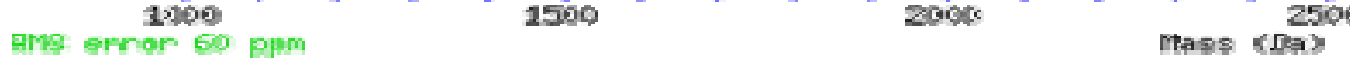




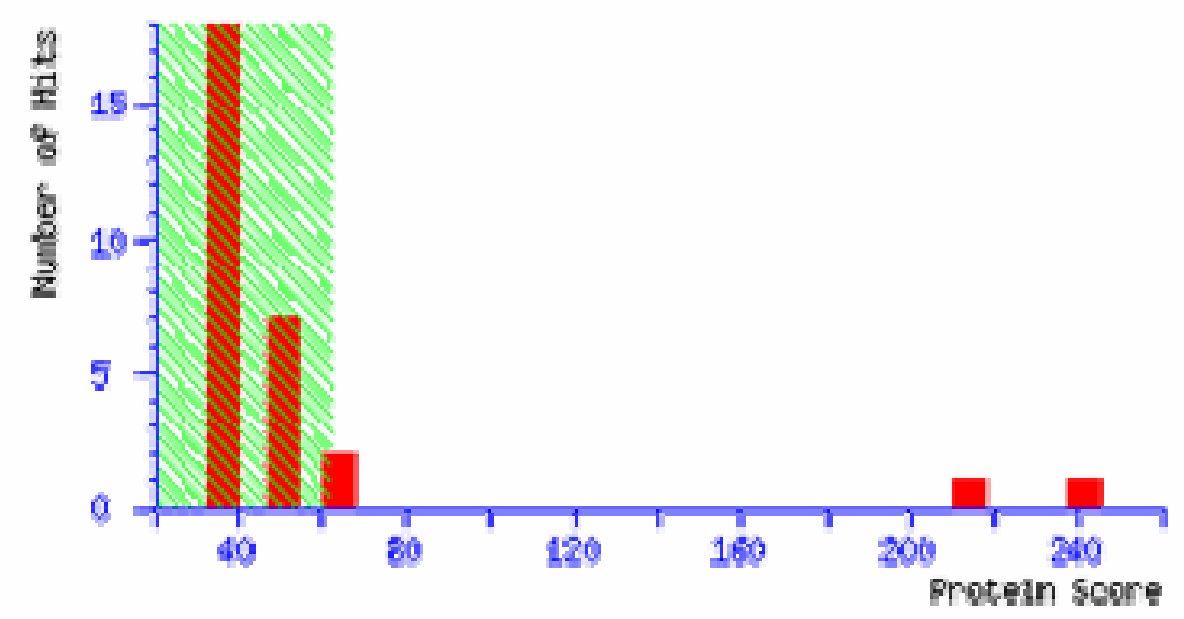

\section{Concise Protein Summary Report}

\section{Format As Concise Protein Summary .}

Significance threshold $\mathrm{p}<0.05 \quad$ Max. number of hits AUTO

Re-Search All

1. VIME MOUSE Mass: 53524 Score: 241

V1ment1n.- Mus musculus (Mouse).

Q5FWJ3 MOUSE Mass: 53655 Score: 241

V1mentin (NOD-derived CD11c +ve dendritic cells cDNA, RIKEN full- length enriched library, clone:F CA.39807 Mass: 53689 Score: 233 Expect: $4.6 e-19$ Matches: 58

MMVMTM NID: - Mus musculus

Q3U6S1_MOUSE Mass: 53641 Score: 232 Expect: 5.8e-19 Matches: 59

Bone marrow macrophage CDNA, RIKEN full-length enriched l1brary, clone:I830119c09 product:v1ment1n O3TFD9 MOUSE Mass: 53656 Score: 230 Expect: 9.2e-19 Matches: 59

17 days embryo heart cDNA, RIKEN full-length enr1ched 11brary, clone: I920087013 product:v1mentin, A.A.40555 Mass: 53641 Score: 229 Expect: $1.2 e-18$ Matches: 58 


\section{Protein View}

Match to: VIME_MOUSE score: 241 Expect: $7.3 e-20$

Vimentin. - Mus musculus (Mouse).

Found $1 \mathrm{n}$ search of pmf_C2_129990848802.txt

Nom1nal mass $\left(M_{x}\right)$ : 53524; calculated pI value: 5.06

NCBI BLAST search of VIME MOUSE aga1nst nr

unformatted sequence str1ng for pasting 1nto other applications

Taxonorny: Mus musculus

Var1able mod1f1cat1ons: Carban1domethyl (C), Dean1dated (NQ), ox1dat1on (M)

cleavage by Trypsin: cuts C-term side of KR unless next residue $1 s$ F

Number of mass values searched: 176

Number of mass values matched: 61

sequence Coverage: $85 \%$

Matched peptides shown 1n Bold Red

1 STRSVSSSSY RRMFGGSGTS SRPSSNRSYV TTSTRTYSLG SALRPSTSRS

51 LYSSSPGGAY VTRSSAVRLR SSVEGVRLLQ DSVDFSLADA INTEFKNTRT

101 NEKVELQELN DRFANYIDKV RFLEQQNKIL LAELEQLKGQ GKSRLGDLYE

151 EEMRELRRQV DQLTNDKARV EVERDNLAED IMRLREKLQE EMLQREEAES

201 TLQSFRQDVD NASLARLDLE RKVESLQEEI AFLKKLHDEE IQELQAQIQE

251 QHVQIDVDVS KPDLTAALRD VRQQYESVAA KNLOEAEEWY KSKFADLSEA

301 ANRNNDALRQ AKQESNEYRR QVQSLTCEVD ALKGTNESLE RQMREMEENF

351 ALEAANYODT IGRLQDEIQN MKEEMARHLR EYODLLNVKM ALDIEIATYR

401 KLLEGEESRI SLPLPTFSSL NLRETNLESL PLVDTHSKRT LLIKTVETRD

451 GQVINETSQH HDDLE

Show predicted peptides also

\section{Sort Peptides By}

$\begin{aligned} \text { Start } & - \text { End } \\ 4 & -12 \\ 12 & -27 \\ 28 & -49 \\ 50 & -63 \\ 78 & -96 \\ 100 & -112 \\ 104 & -112 \\ 113 & -121 \\ 122 & -128 \\ 129 & -138 \\ 129 & -142\end{aligned}$

(9) Residue Number

Observed

1028.5881

1699.9598

2391.3157

1444.7992

2126.1914

1587.8977

1115. 6348

1125. 6698

906.5101

1169.7720

1540.0029

1370.

\section{Mr(expt) Mr (calc)}

$1027.5808 \quad 1027.5047$

$1698.9525 \quad 1698.7856$

$2390.3084 \quad 2390.2190$

$1443.7919 \quad 1443.6994$

$2125.1841 \quad 2125.0579$

$1586.8904 \quad 1586.7900$

$1114.6275 \quad 1114.5618$

$1124.6625 \quad 1124.5978$

$905.5028 \quad 905.4607$

$1168.7647 \quad 1168.7067$

$\begin{array}{ll}1538.9956 & 1538.9032\end{array}$ 


\section{M3}

4700 MS/MS Precursor 1295.75 Spec \#1 MC[BP = 175.1, 1640]








\section{Peptide Summary Report}

\section{Format As Peptide Summary}

Significance threshold $\mathrm{p}<0.05$

Standard scoring

Show pop-ups Suppress pop-ups
Max. number of hits AUTO

Ions score or expect cut-off 0

Sort unassigned Decreasing Score
Help

Show sub-sets 0

Require bold red

\section{Select All}

\section{Select None}

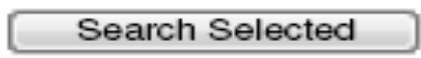

\section{$\square$ Error tolerant} v1mentin.- Mus musculus (Mouse).

$\square$ Check to 1 nclude this hit $1 \mathrm{n}$ error tolerant search

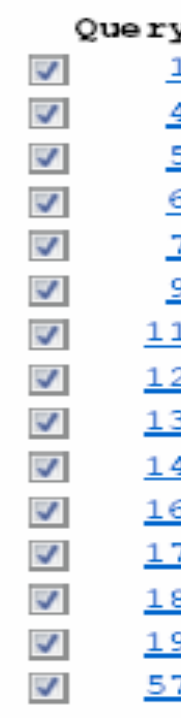

$\begin{array}{rrr}\text { Observed } & \text { Mr(expt) } & \text { Mr(calc) } \\ 932.5250 & 931.5177 & 931.4610 \\ 1081.5635 & 1080.5562 & 1080.4948 \\ 1093.5925 & 1092.5852 & 1092.5200 \\ 1115.6348 & 1114.6275 & 1114.5618 \\ 1125.6698 & 1124.6625 & 1124.5978 \\ 1254.6456 & 1253.6383 & 1253.5598 \\ 1287.7448 & 1286.7375 & 1286.6579 \\ 1295.7462 & 1294.7389 & 1294.6591 \\ 1309.6862 & 1308.6789 & 1308.5986 \\ 1444.7992 & 1443.7919 & 1443.6994 \\ 1557.9950 & 1556.9877 & 1556.8926 \\ 1587.8977 & 1586.8904 & 1586.7900 \\ 1688.9358 & 1687.9285 & 1687.8199 \\ 1734.9248 & 1733.9175 & 1733.8076 \\ 2201.1191 & 2200.1118 & 2199.9742\end{array}$

$\begin{array}{ccc}\text { Ppm } & \text { Miss } & \text { Score } \\ 60.9 & 0 & 37 \\ 56.9 & 1 & 13 \\ 59.7 & 0 & 44 \\ 58.9 & 0 & 30 \\ 57.5 & 1 & 27 \\ 62.7 & 0 & 58 \\ 61.9 & 1 & 11 \\ 61.7 & 0 & 33 \\ 61.4 & 0 & 32 \\ 64.1 & 0 & 29 \\ 61.1 & 0 & 56 \\ 63.3 & 1 & 66 \\ 64.4 & 1 & 23 \\ 63.4 & 1 & 69 \\ 62.6 & 0 & 87\end{array}$

Expect Rank Unique 0.0711

121

0.0111

0. 291

0.321

0.00051

201

0.091

0.161

0.291

0. 000141

6. $7 e-051$

1. 51

$4 e-051$

6. $8 e-07 \quad 1$

\section{Peptide}

K. LLEGEESR. I

K. QESNEYRR. $Q$

K. FADLSEAANR . N

K . VELQELNDR . F

R . FANYIDKVR . F

R. LGDLYEEEMR . E

R. QVDQLTNDKAR. V

K. MALDIEIATYR. K

K. NLQEAEEWYK . S

R. SLYSSSPGGAYVTR. S

R. ISLPLPTFSSLNLR. E

R. TNEKVELQELNDR . F

R. VEVERDNLAEDIMR. L

R. LQDEI QNMKEEMAR . H

R. EMEENFALEAANYQDTIGR . L 


\section{Protein View}

Match to: VIME_MOUSE score: 241

Vimentin. - Mus musculus (Mouse).

Found in search of Ppw_c2_129990854302.txt

Nom1nal mass $\left(M_{x}\right)$ : 53524; Calculated pI value: 5.06

NCBI BLAST search of VIME_MOUSE aga1nst nr

unformatted sequence string for pasting into other applications

Taxonony: Mus musculus

Var1able mod1f1cat1ons: Carban1donethyl (C), Dean1dated (NQ), ox1dat1on (M)

cleavage by Trypsin: cuts c-term side of KR unless next residue $1 s \mathrm{~F}$

sequence coverage: $35 \%$

Matched peptides shown 1n Bold Red

1 STRSVSSSSY RRMFGGSGTS SRPSSNRSYV TTSTRTYSLG SALRPSTSRS

51 LYSSSPGGAY VTRSSAVRLR SSVPGVRLLQ DSVDESLADA INTEFKNTRT

101 NEKVELQELN DRFANYIDKV RFLEQQNKIL LAELEQLKGQ GKSRLGDLYE

151 EEMRELRRQV DQLTNDKARV EVERDNLAED IMRLREKLQE EMLQREEAES

201 TLQSFRQDVD NASLARLDLE RKVESLOEEI AFLKKLHDEE IOELOAQIOE

251 QHVQIDVDVS KPDLTAALRD VRQQYESVAA KNLQEAEEWY KSKFADLSEA

301 ANRNNDALRQ AKQESNEYRR QVQSLTCEVD ALKGTNESLE RQMREMEENF

351 ALEAANYQDT IGRLQDEIQN MKEEMARHLR EYQDLLNVKM ALDIEIATYR

401 KLLEGEESRI SLPLPTFSSL NLRETNLESL FLVDTHSKRT LIIKTVETRD

451 GOVINETSQH HDDLE

\section{Show predicted peptides also}

\section{Sort Peptides By}

- Residue Number

\section{Obse rved}

1444.7992

1587.8977

1115.6348

1125. 6698

1254.6456

1287.7448

1688.9358

1309.6862

1093.5925

1081.5635

2201.1191

1734.9248

1295.7462

932.5250

1557.9950

\section{Mr (expt)}

1443.7919

1586.8904

1114.6275

1124.6625

1286.7375

1687.9285

1308.6789

1092.5852

1080.5562

2200.1118

1733.9175

1294.7389

931.5177

1556.9877
1253.6383
Increasing Mass

Mr (calc)
1443.6994
1586.7900
1114.5618
1124.5978
1253.5598
1286.6579
1687.8199
1308.5986
1092.5200
1080.4948
2199.9742
1733.8076
1294.6591
931.4610
1556.8926

Decreasing Mass

ppm
64
63
59
58
63
62
64
61
60
57
63
63
62
61
61

\section{Miss Sequence}

- R. SLYSSSPGGAYVTR.S (Ions score 29)

1 R. TNEKVELQELNDR. F (IONS score 66 )

- K. VELQELNDR.F (IOnS score 30)

1 R. FANYIDKVR. F (IOnS score 27)

- R. LGDLYEEEMR. E (IONS score 58)

1 R. QVDQLTNDKAR.V (Ions score 11)

1 R. VEVERDNLAEDIMR. L (IOns score 23)

- K. NLQEAEEWYK. S (IONS score 32)

- K. FADLSEAANR. N (Ions score 44)

1 K. QESNEYRR.Q (IOnS score 13)

- R. EMEENF ALEAANYQDTIGR. L (IONS scor

1 R. LQDEIQNMREEMAR. H (I Ons score 69)

- K. MALDIEIATYR.K (Ions score 33)

- K. LLEGEESR. I (IONS score 37 )

o R. ISLPLPTFSSLNLR. E (Ions score 56) 


\section{Sample M4}

4700 Reflector Spec \#1 MC=>NR(2.00)=>NR(2.00)[BP = 1060.6, 21103]

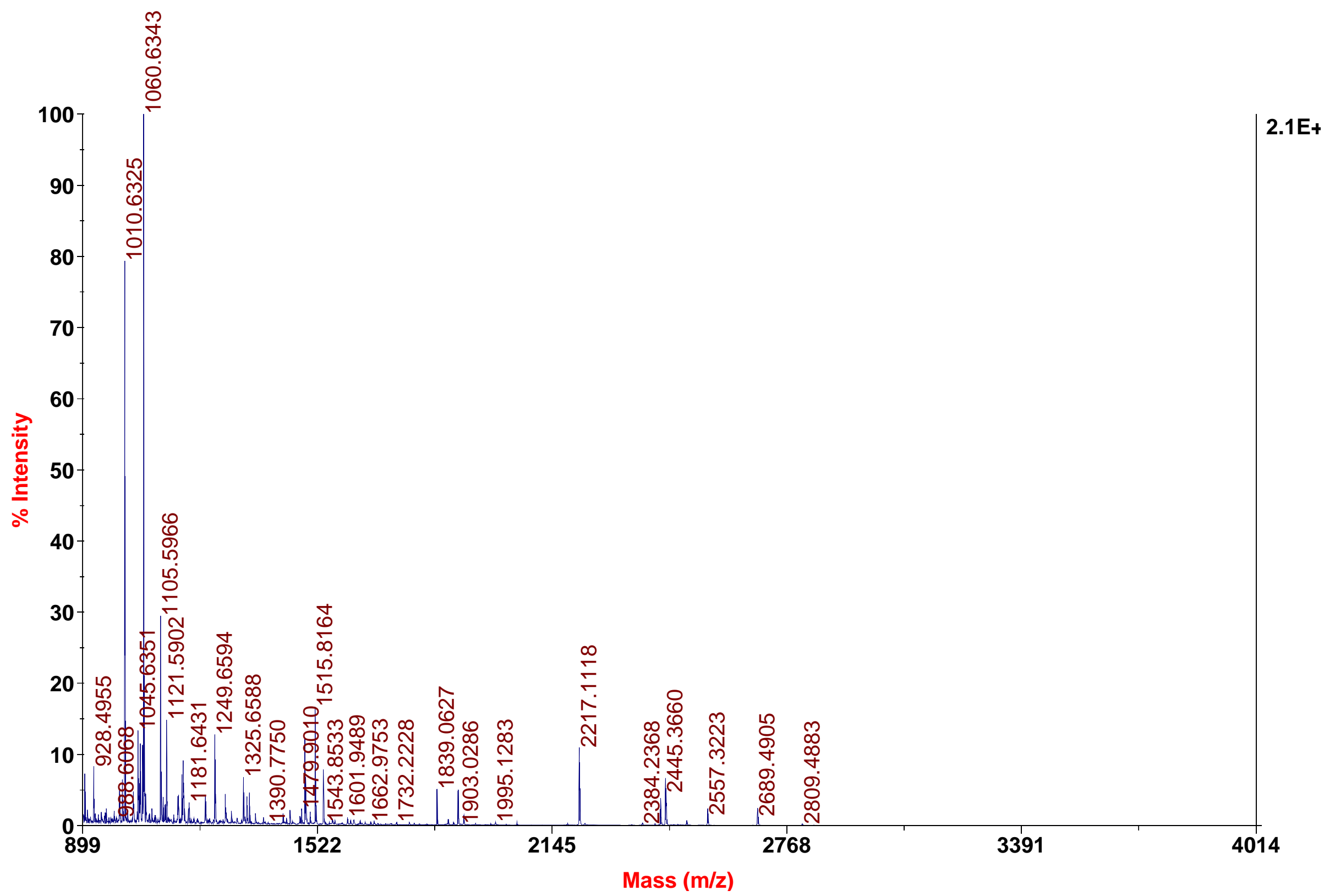




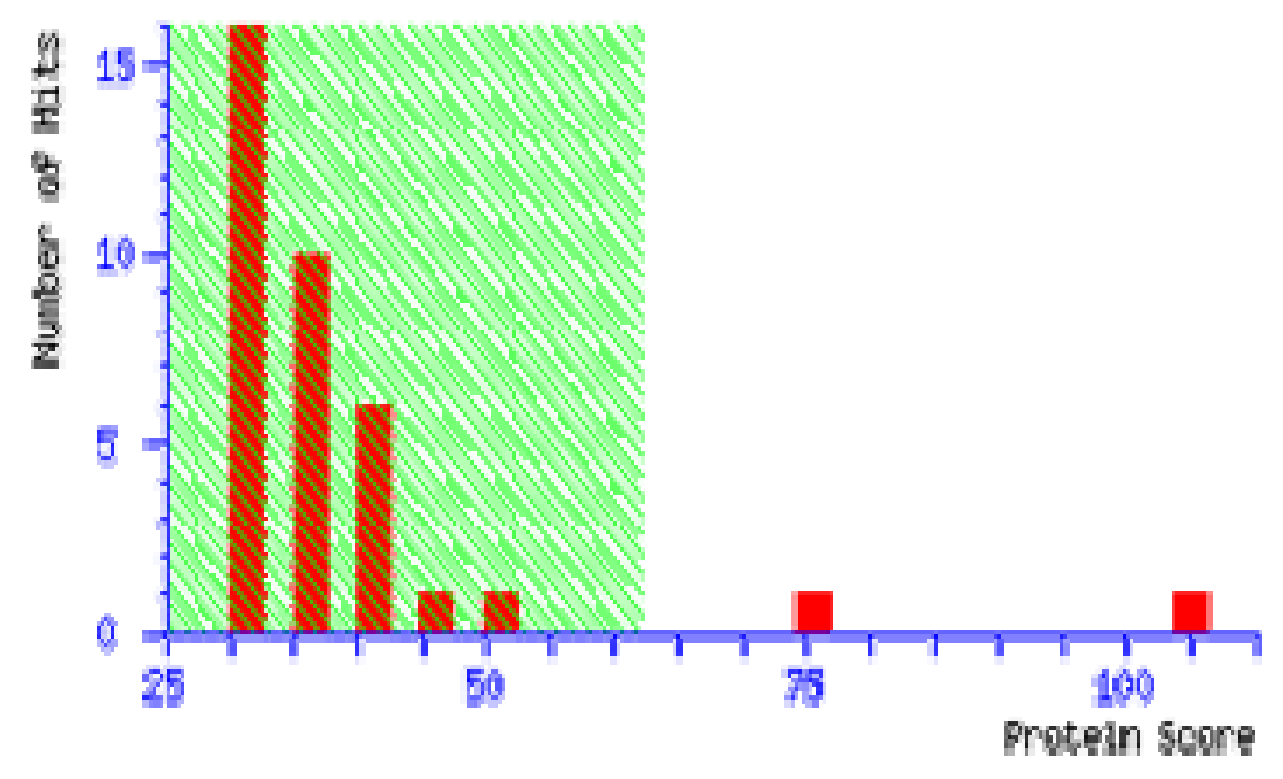

Concise Protein Summary Report

$\begin{array}{rll}\text { Format As Concise Protein Summary } & \text { Help } \\ \text { Significance threshold } p<\overline{0.05} & \text { Max. number of hits AUTO }\end{array}$
Re-Search All
Search Unmatched

1. ARH18158 Mass: 47868 Score: 105 Expect: 2.9e-06 Matches: 28 BC018158 NID: - Mus musculus Q659I1 MOUSE Mass: 53172 Score: 94 Expect: 3.9e-05 Matches: 27 HMW kininogen-I variant (High nolecular weight kininogen I isoform Deltad5). - Mus muscl 


\section{Protein View}

Match to: AAH18158 score: 105 Expect: 2.9e-06 BCO18158 NID

- Mus musculus

Found $1 \mathrm{n}$ search of pmf_D2_129990848803.txt

Nom1nal mass $\left(M_{I}\right)$ : 47868; Calculated pI value: 5.74

NCBI BLAST search of AAH18158 aga1nst nr

unformatted sequence str1ng for pasting 1nto other applications

Taxonorny: Mus musculus

L1nks to retrieve other entries contaln1ng this sequence from NCBI Entrez:

(no taxonomy information for this entry)

Var1able mod1f1cat1ons: Carban1domethyl (C), Dean1dated (NQ), ox1dation

cleavage by Trypsin: cuts c-term side of KR unless next residue $1 s \mathrm{~F}$

Number of mass values searched: 147

Number of mass values matched: 28

sequence coverage: 568

Matched peptides shown 1n Bold Red

1 MkLITtLLLC SGLLLTLTQG EEAQEIDCND EAVFQAVDFS LKQFNFGVKS

51 GNOYMLHRVI EGTKTDGSPT FYSEKYLIKE GNCSAQSGLA WODCDFKDAF

101 EAATGECTAT VGKRENEFEI VTQTCKIAPS KAPILKAYFP CIGCVHAIST

151 DSPDLEPVLK HSIEHENNNT DHSHLFTLRK VKSAHROVVA GLNFDITYTI

201 VQTNCSKERF PSLHGDCVAL PNGDDGECRG NLFMDINNKI ANESQSCTLY

251 SGDDLVEALP KPCPGCPRDI PVDSPELKEV LGHSIAQLNA ENDHPEYYKI

301 DTVKKATSQV VAGTKYVIEF IARETKCSKE SNTELAEDCE IKHLGQSLDC

351 NANVYMRPWE NKVVPTVKCQ ALDMTEMARR PPGFSPFRSV TVOETKEGRT

401 RLLRACEYKG RLSKAGAEPA PERQAESSQV KQ

Show predicted peptides also

\section{Sort Peptides By}

Start - End
$50-58$
$50-58$
$65-75$
$98-114$
$114-126$
$115-126$
$137-160$
$161-180$
$208-229$
$208-229$
$210-229$

Obse rved

1105.5961

1121.5890

1249.6566

1765.9282

1671.9396

1515.8158

2689.4998

2449.3303

2501.2832

2502.2778

2216.1101

\section{Mr (expt) Mr (calc)}

1104.5888

1120,5817

1248.6493

1764.9209

1670.9323

1514.8085

2688.4925

2448.3230

2500.2759

2501.2705

2215.1028

Mr (calc)
1104.5134
1120.5084
1248.5663
1764.7948
1670.8086
1514.7075
2688.2928
2448.1570
2500.0860
2501.0700
2214.9423

Decreasing Mass

ppm
68
65
67
71
74
67
74
68
76
80
72

Miss Sequence

o K. SGNQYMLHR.V

O K. SGNOYMILHR V V

O K. TDGSPTEYSEK. $Y$

. Carba

o R. ENEFFIVTQTCK. I Carbarn1dor

K. AYFPCIGCVHA ISTDSPDLEPVLK .

1 K. HSIEHFNINNTDHSHLETLRK.V 2

1 K. ERFPSLHGDCVA LPNGDDGECR.G

1 K. ERFPSLHGDCVA LPNGDDGECR.G

o R. FPSLHGDCVALPNGDDGECR.G 2 


\section{M4}

4700 MS/MS Precursor 1045.63 Spec \#1 MC[BP = 860.9, 1921]

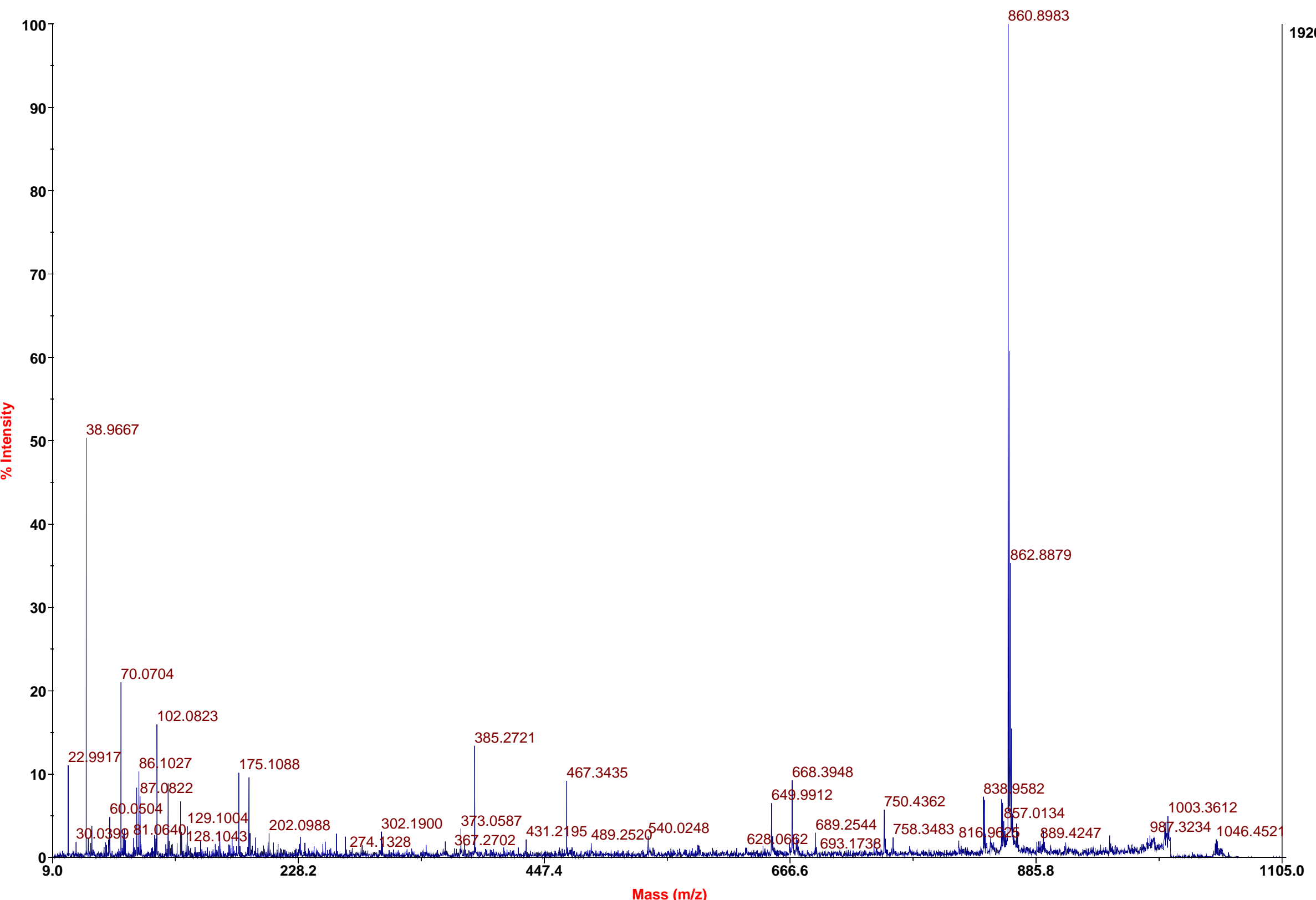




\section{M4}

4700 MS/MS Precursor 1004.56 Spec \#1 MC[BP = 1010.3, 2869]

곤

100

70

60

0

10

\begin{tabular}{r|r|}
59.0557 \\
60.0548
\end{tabular} \mid$\quad 235.1542$

$\begin{array}{lll}100.0888 & 158.0943 \quad 229.1289\end{array}$



9.0

219.4

175.1116

506.2776

$314.2081 \quad 390.1917$

$\begin{array}{lll}314.2081 & 489.2613 \\ 301.1978 & 372.1671 & 459.2498\end{array}$

1010.2844

2868.6

560.3262

2648 $626.2786 \quad 703.3243 \quad 765.3248$

847.3638 $873.3271 \quad 948.2933$ 


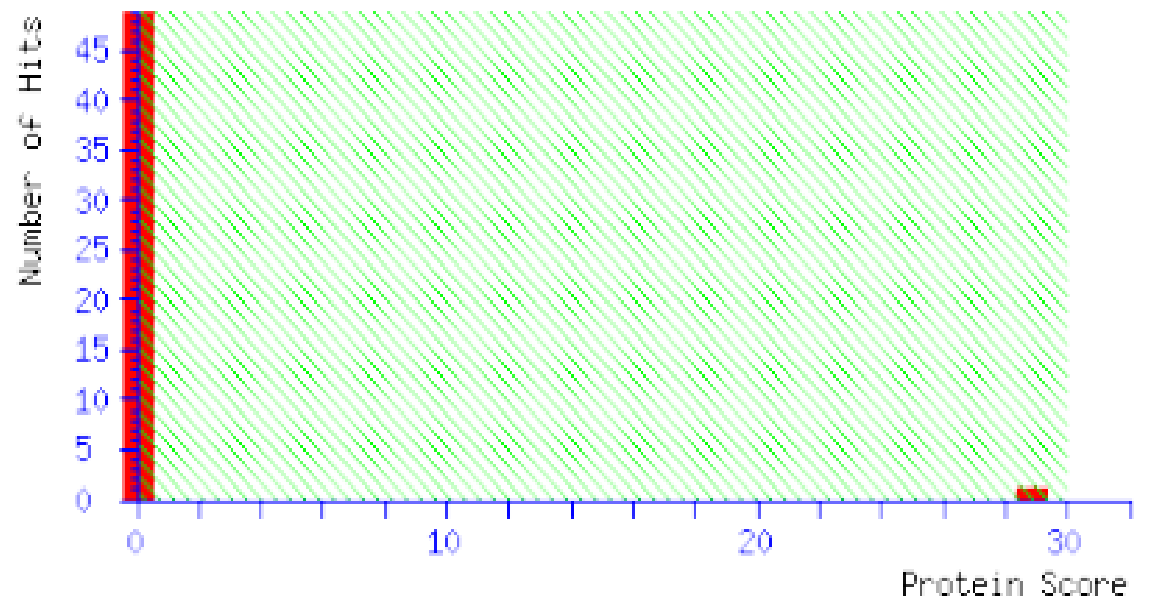

\section{Peptide Summary Report}

RNG1 MoUSE Mass: 73056 Score: 29 Matches: 5

(0) Sequences: $5(0)$ emPAI: 0.18

Rininogen-1 OS=Mus musculus GN=Kng1 $\mathrm{PE}=1 \mathrm{SV}=1$

$\square$ Check to include this hit in error tolerant search

\begin{tabular}{|c|c|c|c|c|c|c|c|c|c|c|}
\hline uery & Observed & $\operatorname{Mr}(\operatorname{expt})$ & $\operatorname{Mr}(\mathrm{calc})$ & ppm & Miss & Score & Expect & Rank & Unique & Peptide \\
\hline$\underline{4}$ & 1010.6306 & 1009.6233 & 1009.5596 & 63.1 & 0 & 18 & 0.33 & 1 & U & K.YVIEFIAR. E \\
\hline$\underline{9}$ & 1112.6533 & 1111.6460 & 1111.5761 & 62.9 & 0 & 11 & 3.5 & 1 & U & R.DIPVDSPELK,E \\
\hline 12 & 1249.6566 & 1248.6493 & 1248.5663 & 66.5 & 0 & 18 & 1.4 & 1 & u & K. TDGSPTFYSFK. Y \\
\hline$\underline{13}$ & 1325.6593 & 1324.6520 & 1324.5574 & 71.5 & 0 & 17 & 1.4 & 1 & U & $\mathrm{K} \cdot \mathrm{CQALDMTEMAR} \cdot \mathrm{R}+$ Carbamidomethyl (C) \\
\hline$\underline{47}$ & 2216.1101 & 2215.1028 & 2214.9423 & 72.5 & 0 & 30 & 0.088 & 1 & u & R. FPSLHGDCVALPNGDDGECR, $G+2$ Carbamido \\
\hline
\end{tabular}




\section{Protein View}

Match to: KNG1_MOUSE Score: 29

Kininogen-1 OS=Mus musculus $\mathrm{GN}=\mathrm{Kng} 1 \mathrm{PE}=1 \mathrm{SV}=1$

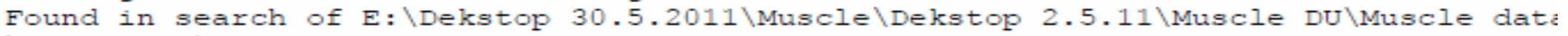
$\backslash$ sample M4 \ppw_D2_129990854403.txt

Nominal mass $\left(M_{Y}\right): 73056$; Calculated pI value: 6.05

NCBI BLAST search of KNG1 MOUSE against nr

Unformatted sequence string for pasting into other applications

Taxonomy: Mus musculus

Variable modifications: Carbamidomethyl (C), Deamidated (NQ), Oxidation (M)

Cleavage by Trypsin: cuts C-term side of KR unless next residue is $P$

Sequence Coverage: 98

Matched peptides shown in Bold Red

1 MRIITTLLC SGLLITLTQG EEAQEIDCND EAVFQAVDFS LKQFNPGVKS

51 GNQYMLHRVI EGTKTDGSPT FYSFKYLIKE GNCSAQSGLA WQDCDFKDAE

101 EAATGECTAT VGKRENEFFI VTQTCKIAPS KAPILKAYFP CIGCVHAIST

151 DSPDLEPVLK HSIEHFNNNT DHSHLFTLRK VRSAHRQVVA GLNFDITYTI

201 VQTNCSKERF PSLHGDCVAL PNGDDGECRG NLFMDINNKI ANFSQSCTLY

251 SGDDLVEALP RPCPGCPRDI PVDSPELKEV LGHSIAQLNA ENDHPFYYKI

301 DTVKKATSQV VAGTKYVIEF IARETKCSKE SNTELAEDCE IKHLGQSLDC

351 NANVYMRPWE NKVVPTVKCQ ALDMTEMARR PPGFSPFRSV TVQETKEGRT

401 VSPRYIAREQ EERDAETEQG PTHGHGWLHE KQIKANKNHR GHKHGHDHGH

451 WSPRRHGLGH GHQKPHGLGH GHQLKLDYLR HQREDGDDHT HTVGHGHGHG

501 HGHGHGHGHG HGHGHGHGHG HGHGKHTNKD KNSVKQTTQR TESLASSSEY

551 STTSTQMQGR TEGPTITPPR AQPTVTSSGF QDSDFIEDVV ATTPPYDTGA

601 HDDLIPDIHV QPDSLSFKII SDFPEATSPK CPGRPWKPAS WEDPNTETTE

651 FSDFDLLDAL S

\section{Show predicted peptides also}

\section{Sort Peptides By}

\section{Start - End \\ $65-75$}

$\frac{(\text { Ions score } 18 \text { ) }}{210-229}$

2216.1101

Observed

○ Residue Number

O Increasing Mass

Decreasing Mass

\author{
FPSLHGDCVALPNGDDGECR . G
}

\section{Mr (expt) Mr (calc) \\ $1248.6493 \quad 1248.5663$}

$2215.1028 \quad 2214.9423$

2 Carbamidomethyl

\author{
(c) (Ions score 30 )
}

Miss Sequence

0 K. TDGSPTFYSFK. Y

o R. 


\section{Sample M5}

4700 Reflector Spec \#1 MC=>NR(2.00)[BP = 1003.6, 11606]

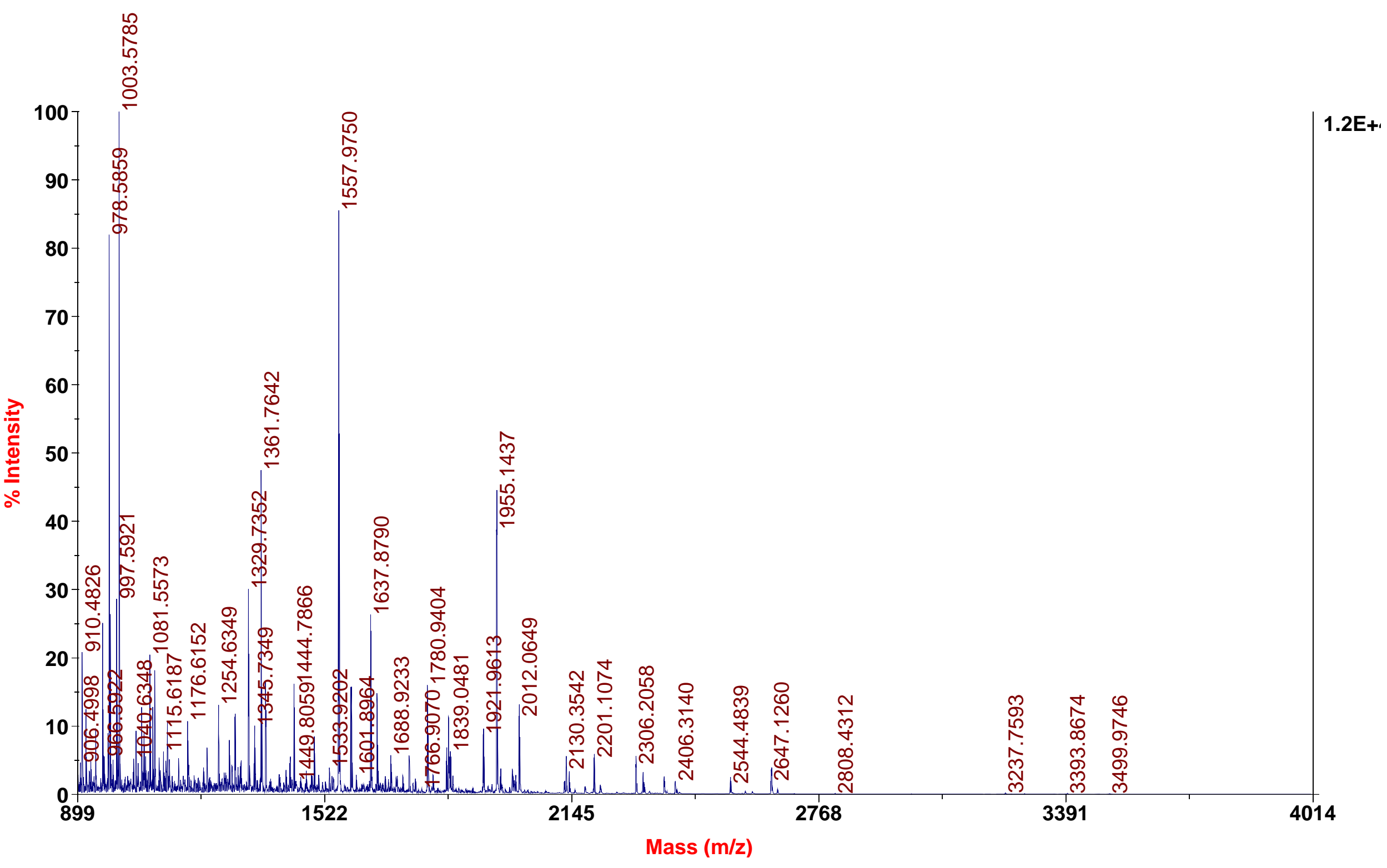




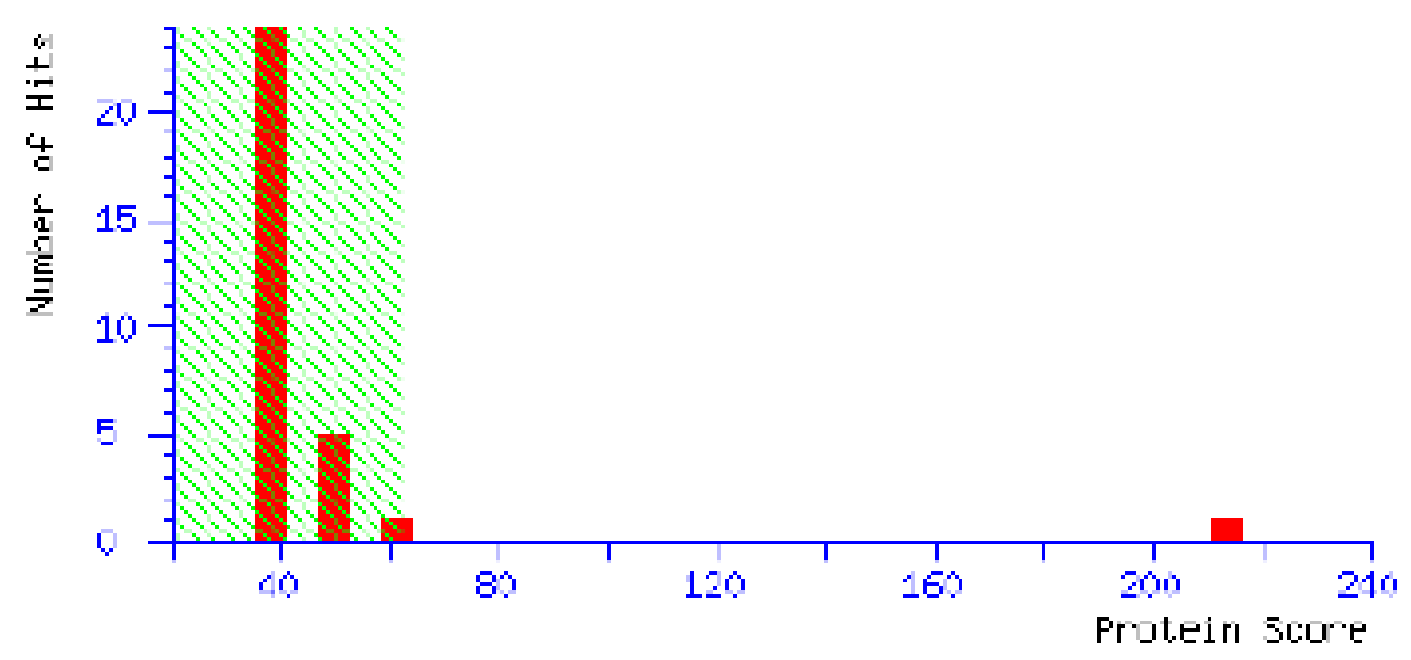

\section{Concise Protein Summary Report}

\section{Format As}

\section{Concise Protein Summary}

Significance threshold $\mathrm{p}<$

$$
0.05
$$

Help

Max. number of hits

AUTO

\section{Re-Search All}

\section{Search Unmatched}

CAR69019 Mass: 51533 Score: 214

Expect: $3 \cdot 7 e-17$

Matches: 23

MMVIMENT NID:

VIME_MOUSE Mass: 53524 Sco

A43803 Mass: 53597 Score: 211 Expect: $7.3 e-17$ Matches: 23

vimentin - mouse

Q5FWJ3_MOUSE Mass: 53655 Score: 211 Expect: $7.3 e-17$ Matches: 23 


\section{Protein View}

Match to: CAA69019 Score: 214 Expect: $3.7 e-17$

MIVIMENT NID : - Mus musculus

Found in search of E: VDekstop 30.5.2011 Muscle J sample M5

vomf F19_130406586900.txt

Nominal mass $\left(M_{Y}\right)$ : 51533; Calculated pI value: 4.96

NCBI BLAST search of CAA69019 against nr

Unformatted sequence string for pasting into other applications

Taxonomy: Mus musculus

Cleavage by Trypsin: cuts C-term side of KR unless next residue is P

Number of mass values searched: 60

Number of mass values matched: 23

Sequence Coverage: 518

Matched peptides shown in Bold Red

1 SSRPSSNRSY VTTSTRTYSL GSALRPSTSR SLYSSSPGGA YVTRSSAVRL

51 RSSVPGVRIL QDSVDFSIAD AINTEFKNTR TNEKVELQEL NDRFANYIDK

101 VRELEQQNKI LLAELEQLKG QGKSRLGDIY EEEMRELRRQ VDQLTNDKAR

151 VEVERDNLAE DIMRLREKLQ EEMLQREEAE STLQSFRQDV DNASIARLDI

201 ERKVESLQEE IAFLKKLHDE EIQELQAQIQ EQHVQIDVDV SKPDLTAALR

251 DVRQQYESVA AKNLQEAFEW YKSKFADLSE AANRNNDALR QAKQESNEYR

301 RQVQSLTCEV DALKGTNESI ERQMREMEEN FALEAANYQD TIGRIQDEIQ

351 NMKEEMARHL REYQDLLNVK MALDIEIATY RKLLEGEESR ISLPLPTFSS

401 LNLRETNLES LPLVDTHSKR TLIIKTVETR DGQVINETSQ HHDDLE

Show predicted peptides also

Sort Peptides By

Start - End

$17-30$

S

s

$$
31-44
$$

$81-93$

$85-93$

$94-102$

$126-135$

$140-150$

$151-164$

- Residue Number

\section{Increasing Mass}

Decreasing Mass

Observed 1495.8473

1444.7740

1587.8723

1115.6179

1125.6492

1254.6244

1287.7262

1688.9004

\section{Mr (expt)}

1494.8400

1443.7667

1586.8650

1114.6106

1124.6419

1253.6171

1286.7189

1687.8931
$\operatorname{Mr}(\operatorname{calc})$

1494.7790

1443.6994

1586.7900

1114.5618

1124.5978

1253.5598

1286.6579

1687.8199 ppm

41

47

47

44

39

46

47

43

\section{Miss Sequence}

o R. TYSLGSALRPSTSR.

o R. SLYSSSPGGAYVTR.

1 R. TNEKVELQELNDR . F

K . VELQELNDR . F

1 R. FANYIDKVR . F

o R. LGD LYEEEMR . E

1 R. QVDQLTNDKAR.V

1 R. VEVERDNI.AED IMR. 


\section{M5}

$4700 \mathrm{MS} / \mathrm{MS}$ Precursor 978.586 Spec \#1 MC[BP = 70.1, 2864]

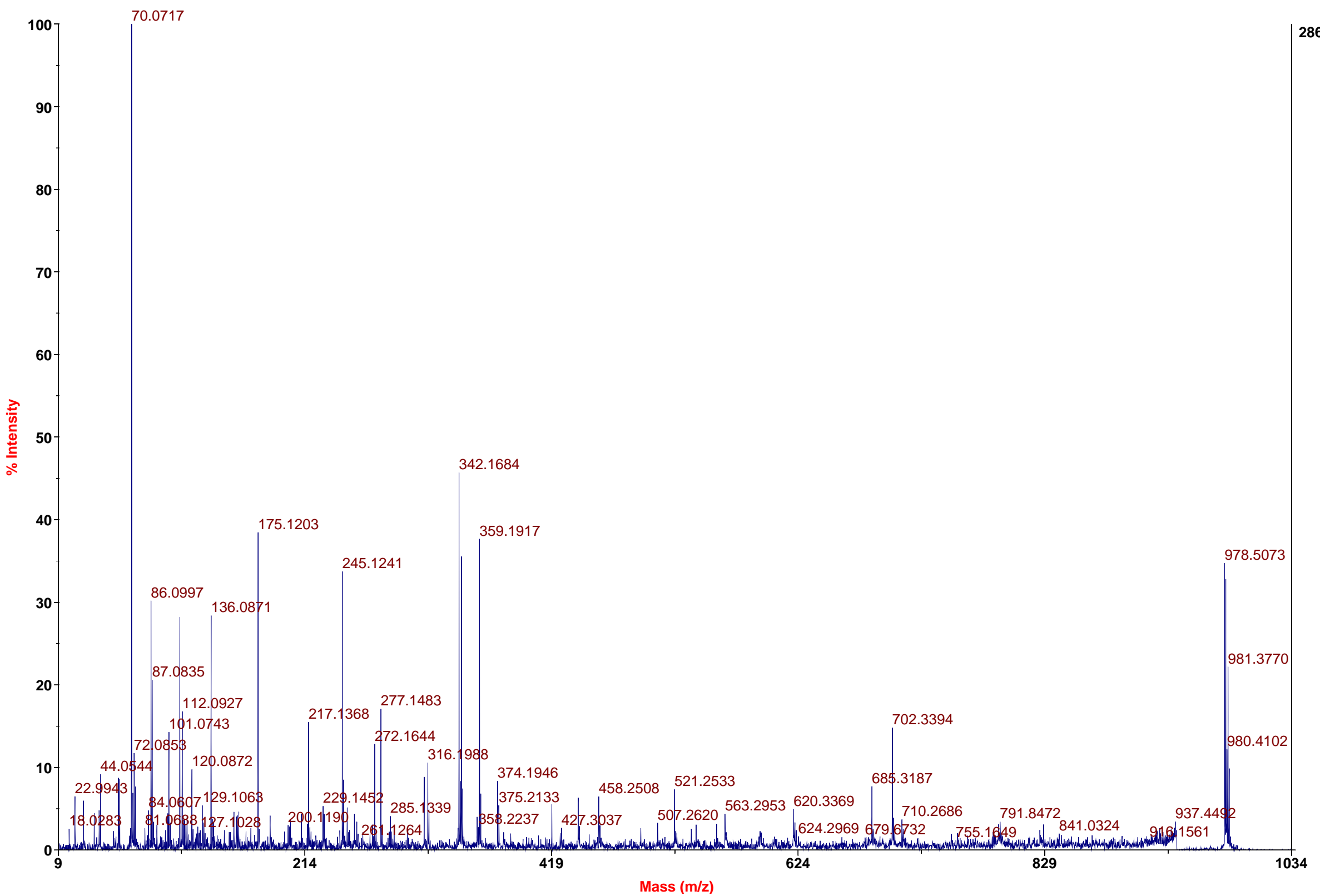




\section{M5}

4700 MS/MS Precursor 1556.96 Spec \#1 MC[BP = 86.1, 1687]






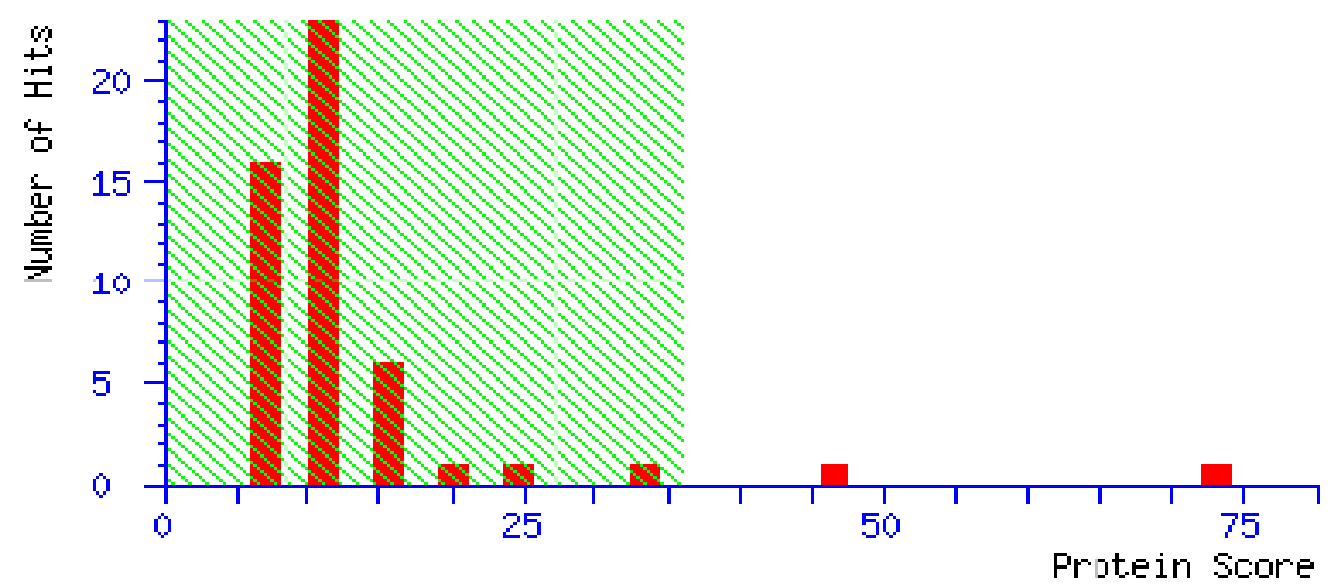

\section{Peptide Summary Report}

VIME NOUSE Mass: 53524 Score: 73 Matches: $3(0)$ Sequences: $3(0)$

Vimentin.- Mus musculus (Mouse).

$\square$ check to include this hit in error tolerant search

Query Observed Mr(expt) Mr(calc) ppm Miss Score Expect Rank Unique Peptide

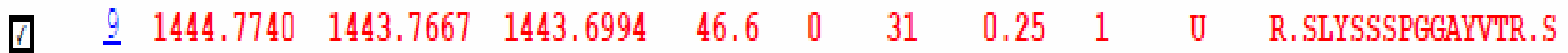

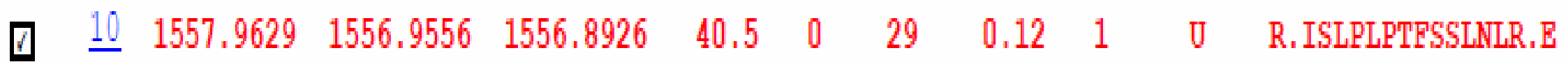

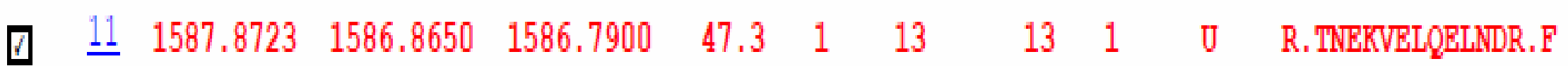




\section{Protein View}

Match to: VIME_MOUSE Score: 73

Vimentin. - Mus musculus (Mouse).

Found in search of E: \Dekstop 30.5.2011 Muscle

\PPW_F19_130406591600.txt

Nominal mass $\left(M_{Y}\right)$ : 53524 ; Calculated pI value: $\mathbf{5 . 0 6}$

NCBI BLAST search of VIME MOUSE against nr

Unformatted sequence string for pasting into other applications

Taxonomy: Mus musculus

Variable modifications: Carbamidomethyl (C), Deamidated (NQ), Oxidation cleavage by Trypsin: cuts c-term side of KR unless next residue is p

sequence Coverage: 88

Matched peptides shown in Bold Red

1 STRSVSSSSY RRMFGGSGTS SRPSSNRSYV TTSTRTYSLG SALRPSTSRS

51 LYSSSPGGAY VTRSSAVRLR SSVPGVRLLQ DSVDFSLADA INTEFKNTRT

101 NEKVELQELN DRFANYIDKV RFLEQQNKIL LAELEQLKGQ GKSRLGDLYE

151 EEMRELRRQV DQLTNDKARV EVERDNLAED IMRLREKLQE EMLQREEAES

201 TLQSFRQDVD NASLARLDLE RKVESLQEEI AFLKKLHDEE IQELQAQIQE

251 QHVQIDVDVS KPDLTAALRD VRQQYESVAA KNLQEAEEWY KSKEADLSEA

301 ANRNNDALRQ AKQESNEYRR QVQSLTCEVD ALKGTNESLE RQMREMEENE

351 ALEAANYQDT IGRLQDEIQN MKEEMARHLR EYQDLINVKM ALDIEIATYR

401 KLLEGEESRI SLPLPTFSSL NLRETNLESL PLVDTHSKRT ILIKTVETRD

451 GQVINETSQH HDDLE

\section{Show predicted peptides also}

\section{Sort Peptides By}

\section{Start - End $50-63$ \\ Observed 1444.7740 \\ S \\ (Ions score 31 )

$$
100-112
$$

1587.8723

(Ions score 13)
$410-423$
1557.9629

Mr (expt)

1443.7667

1586.8650

1556.9556
Mr (calc) 1443.6994

1586.7900

1556.8926

\section{Increasing Mass \\ Residue Number}

ppm 
Sample M6

4700 Reflector Spec \#1 MC=>NR(2.00)[BP = 980.5, 23361]

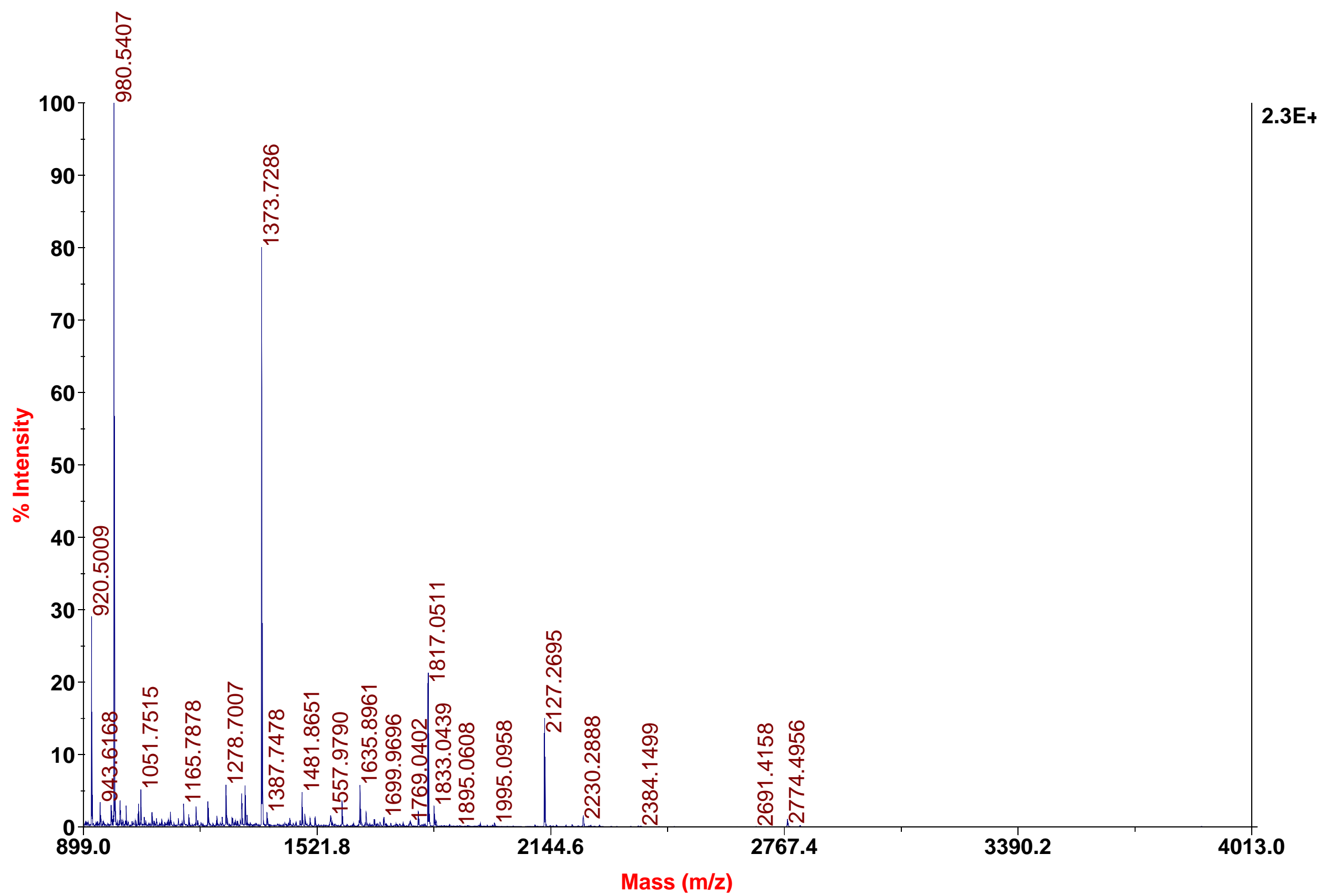




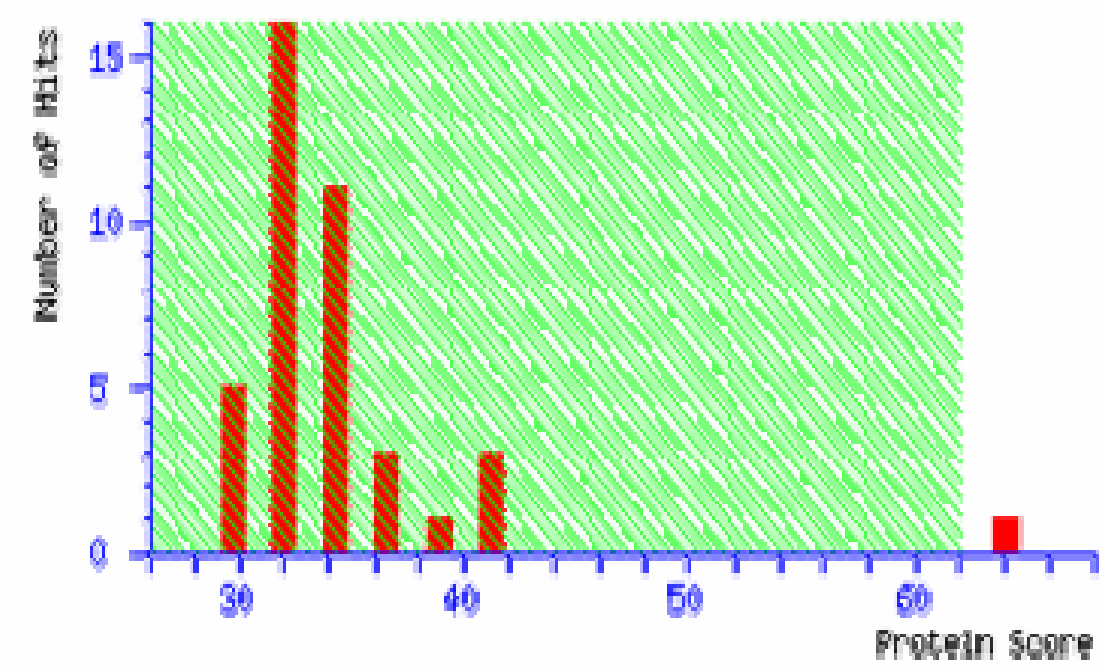

\section{Concise Protein Summary Report}

Format As Concise Protein Summary . Help

Significance threshold $p<\longdiv { 0 . 0 5 }$ Max. number of hits AUTO

\section{Re-Search All Search Unmatched}

1. Q3UBS3 MOUSE Mass: 38727 Score: 64 Expect: 0.036 Matches: 21

Bone marrow macrophage CDNA, RIKEN full-length enriched l1brary, clone: I830015M19 product:haptoglobin, 063927 9MURI Mass: 38750 Score: 53 Expect: 0.48 Matches: 19 Haptoglobin.- Mus sp.

HPMS Mass: 38821 Score: 52 Expect: 0.54 Matches: 19

haptoglobin precursor - mouse 


\section{Protein View}

Match to: Q3UBS3_MOUSE score: 64 Expect: 0.036

Bone marrow macrophage CDNA, RIKEN full-length enriched library, clone: I830015M19 product:haptoglobin, Found in search of pmf_F2_129990848905.txt

Nom1nal mass $\left(M_{x}\right): 38727$; Calculated pI value: 5.88

NCBI BLAST search of Q3UBS3_MOUSE aga1nst $\mathrm{nr}$

unformatted sequence string for pasting into other applications

Taxonorny: Mus musculus

Links to retrieve other entries contalning this sequence from NCBI Entrez:

(no taxonomy information for this entry)

(no taxonomy information for this entry)

(no taxonomy information for this entry)

(no taxonomy information for this entry)

(no taxonomy information for this entry)

(no taxonomy information for this entry)

(no taxonomy information for this entry)

Var1able mod1f1cat1ons: Carban1domethyl (C), Dean1dated (NQ), ox1dat1on (M) cleavage by Trypsin: cuts c-term. side of KR unless next residue $1 \mathrm{~s} P$

Number of mass values searched: 129

Number of mass values matched: 21

sequence coverage: 54\%

Matched peptides shown 1n Bold Red

1 MRALGAVVTL LLWGQLFAVE LGNDAMDFED DSCPKPPEIA NGYVEHLVRY

51 RCRQFYRLRA EGDGVYTLND EKQWVNTVAG EKLPECEAVC GKPKHPVDQV

101 QRIIGGSMDA KGSFPWQAKM ISRHGLTTGA TLISDQWLLT TAKNLELNHS

151 ETASAKDITP TLTLYVGKNQ LVEIEKVVLH PNHSVVDIGL IKLKORVLVT

201 ERVMPICLPS KDYIAPGRVG YVSGWGRNAN FRFTDRLKYV MLPVADQDKC

251 VVHYENSTVP EKKNLTSPVG VQPILNEHTF CAGLTKYQED TCYGDAGSAF

301 AIHDMEEDTW YAAGILSFDK SCAVAEYGVY VRATDLKDWV QETMAKN

Show predicted peptides also

Sort Peptides By Residue Number Increasing Mass Decreasing Mass

$\begin{aligned} \text { Start } & - \text { End } \\ 83 & -94 \\ 95 & -102 \\ 103 & -119 \\ 112 & -119 \\ 124 & -143 \\ 157 & -168 \\ 169 & -176\end{aligned}$

Obse rved

1387.7445

978.5474

1809.0271

920.5013

2127.2664

1320.8011

972.5767

\section{Mr (expt) Mr (calc)}

1386.7372

977.5401

1808.0198

919. 4940

2126.2591

1319.7938

971.5694
1386.6635

977.5043

1807.8927

919.4552

2126.1372

1319.7337

971.5287

\section{ppm \\ Miss Sequence}

53

37

70

42

57

46

42 o K. LPECEAVCGKPK.H 2 Carbam1donethyl

0 K. HPVDQVQR. I

1 R. IIGGSMDAKGSFPWQAK.M Oxidation (M)

0 K. GSFPWQAK. M

- R. HGLTTGATLISDQWLLTTAK. N

- K. DITPTLTLYVGK.N

O K. NQLVEIEK. V 


\section{M6}

4700 MS/MS Precursor 980.541 Spec \#1 MC[BP = 136.1, 5532]

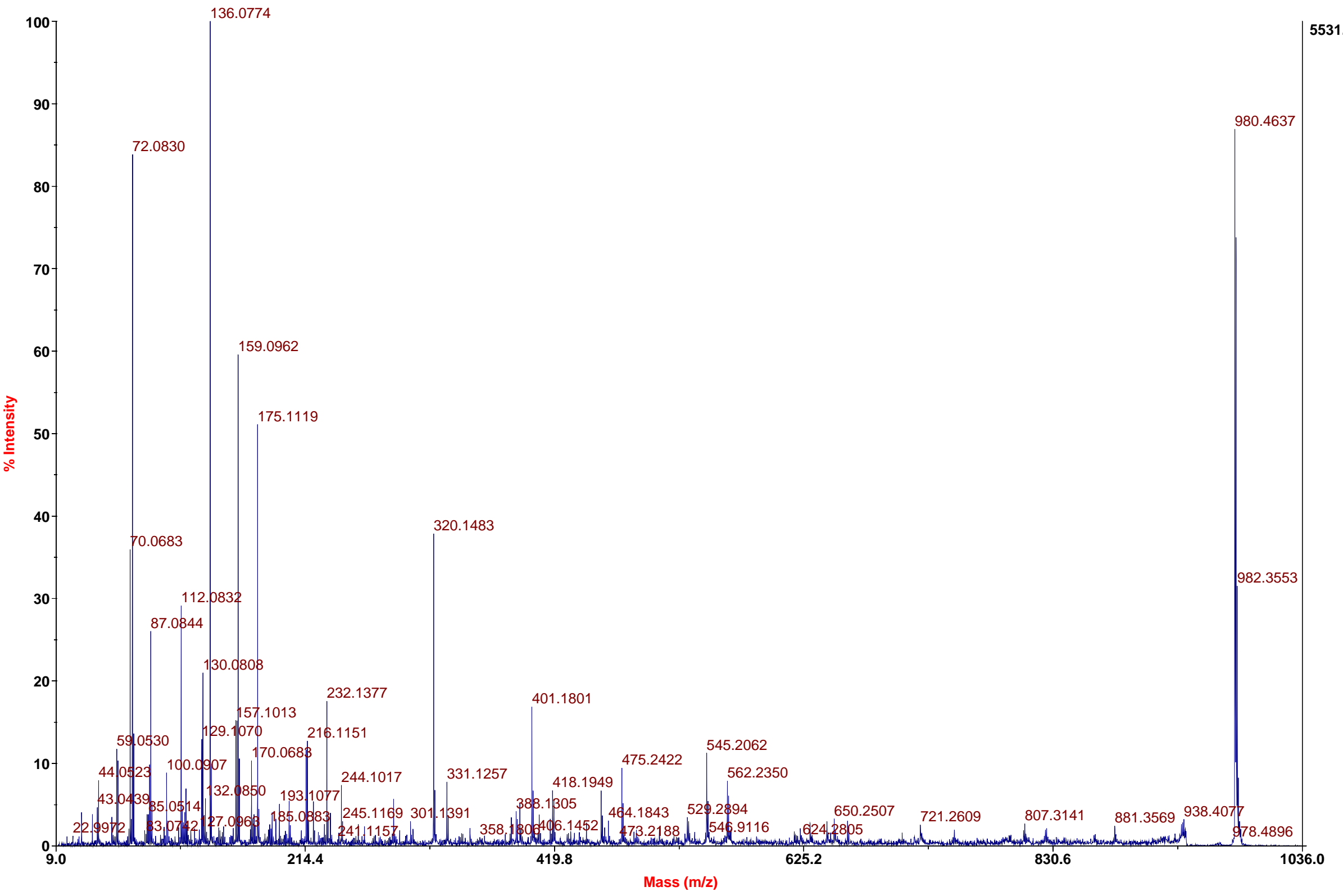




\section{M6}

4700 MS/MS Precursor 1051.75 Spec \#1 MC[BP = 861.0, 2260]

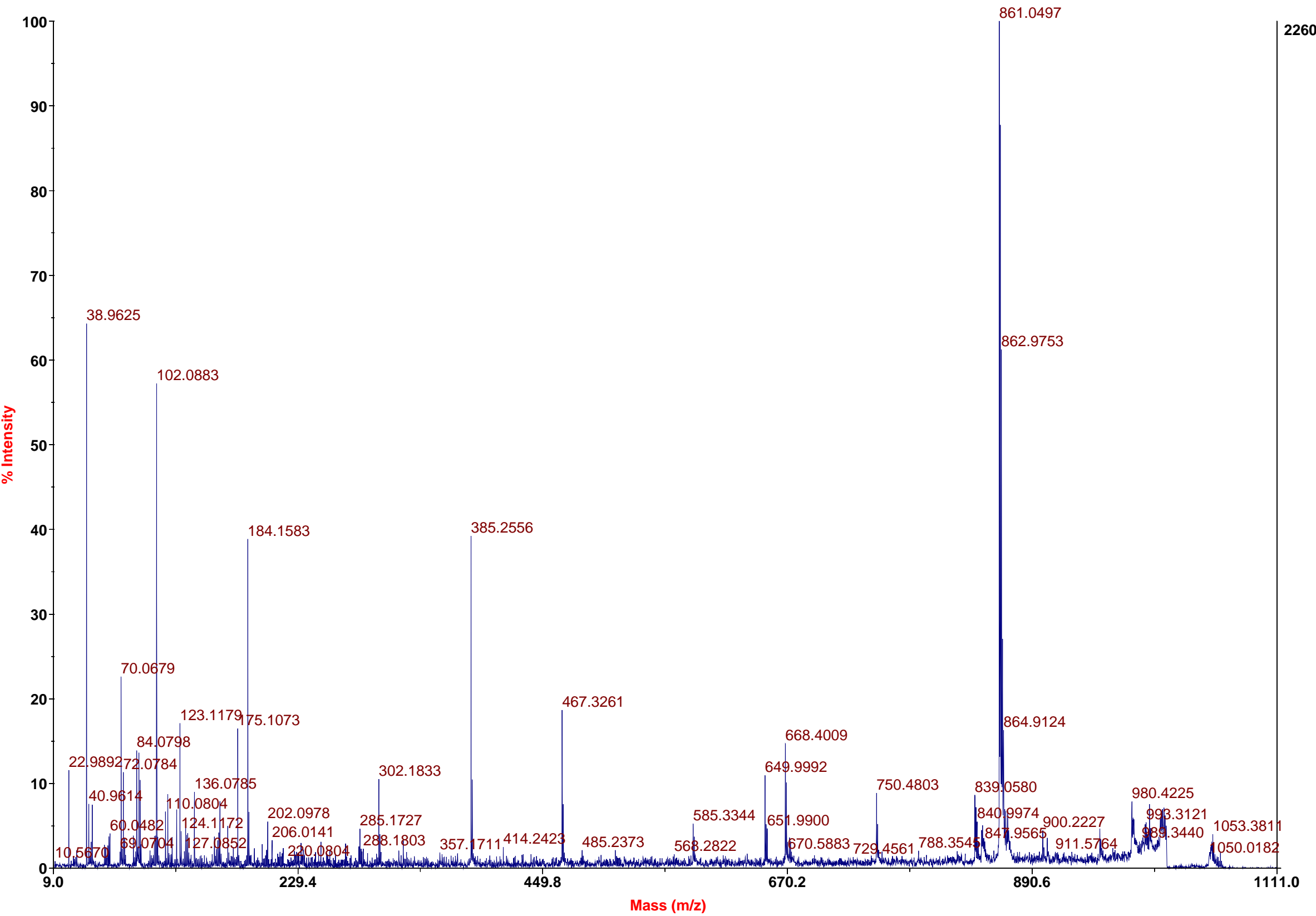






\section{Peptide Summary Report}

Format As Peptide Summary

Significance threshold $\mathrm{p}<0.05$

Standard scoring $\bigcirc$ MudPIT scoring $\odot$ Ions score or expect cut-off 0

Show pop-ups $\odot$ Suppress pop-ups $\bigcirc$ Sort unassigned Decreasing Score
Help

Show sub-sets 0

Require bold red

Search Selected

$\square$ Error tolerant

1. HPMS Mass: 38821 Score: 117 Matches: 9(3) Sequences: 8(3) emPAI: 1.03 haptoglobin precursor - mouse

$\square$ check to 1 nclude this hit in error tolerant search

\begin{tabular}{lrrrr}
\multicolumn{1}{l}{ Query } & $\begin{array}{r}\text { Observed } \\
\text { Q Mr (expt) }\end{array}$ & \multicolumn{1}{c}{ Mr (calc) } \\
$\square$ & $\underline{1}$ & 920.5013 & 919.4940 & 919.4552 \\
$\square$ & $\underline{3}$ & 972.5767 & 971.5694 & 971.5287 \\
$\square$ & $\underline{4}$ & 980.5405 & 979.5333 & 979.4876 \\
$\square$ & $\underline{10}$ & 1278.6979 & 1277.6906 & 1277.6326 \\
$\square$ & $\underline{11}$ & 1320.8011 & 1319.7938 & 1319.7337 \\
$\square$ & $\underline{16}$ & 1635.8950 & 1634.8877 & 1634.7974 \\
$\square$ & $\underline{18}$ & 1817.0505 & 1816.0432 & 1815.9375 \\
$\square$ & $\underline{19}$ & 1833.0441 & 1832.0368 & 1831.9324 \\
$\square$ & $\underline{38}$ & 2127.2664 & 2126.2591 & 2126.1372
\end{tabular}

$\begin{array}{lcc}\text { ppm } & \text { Miss } & \text { Score } \\ 42.2 & 0 & 32 \\ 41.8 & 0 & 24 \\ 46.6 & 0 & 39 \\ 45.4 & 0 & 37 \\ 45.6 & 0 & 23 \\ 55.3 & 1 & 26 \\ 58.2 & 1 & 52 \\ 57.0 & 1 & (22) \\ 57.4 & 0 & 57\end{array}$

Expect Rank Unique $0.17-1$

$0.94 \quad 1$

$0.03 \quad 1$

$0.058 \quad 1$

0.661

$0.76 \quad 1$

0.00131

1.31

$0.00022 \quad 1$

\section{Peptide}

K. GSFPWQAK. M

K. NQLVEIEK. V

R. VGYVSGWGR . N

K. YVMLPVADQDK. C

K. DITPTLTLYVGK. N

R. ATDLKDWVQE TMAK. N

R. VMPICLLPSKDYIAPGR.V + Carl

R. VMPIㄷLPSKDYIAPGR.V + Carl

R. HGLTTGATLISDQWLLTTAK. N 


\section{Protein View}

Match to: HPMS score: 117

\section{haptoglobin precursor - mouse}

Found in search of Ppr_F2_129990854705.txt

Nom1nal mass $\left(\mathrm{M}_{\mathrm{r}}\right)$ : 38821; Calculated pI value: 6.08

NCBI BLAST search of HPMS aga1nst nr

unformatted sequence string for pasting 1nto other applications

Taxonony: Mus musculus

Var1able modif1cations: Carban1domethyl (C), Dean1dated (NQ), ox1dation (M)

cleavage by Trypsin: cuts c-term side of KR unless next residue 1 s $P$

sequence coverage: $28 \%$

Matched peptides shown in Bold Red

1 MRALGAVVTL LLWGQLFAVE LGNDAMDFED DSCPKPPEIA NGYVEHLVRY

51 RCRQFYRLRA EGDGVYTLND EKQWVNTVAG HKLPECEAVC GKPKHPVDQV

101 QRIIGGSMDA KGSFPWQAKM ISRHGLTTGA TLISDQWLLT TAKNLELNHS

151 ETASAKDITP TLTLYVGKNQ LVEIEKVVLH PNHSVVDIGL IKLKQRVLVT

201 ERVMPICLPS KDYIAPGRVG YVSGWGRNAN FRFTDRLKYV MLPVADQDKC

251 VVHYENSTVP EKKNLTSPVQ VQPILNEHTF CAGLTKYQED TCYGDAGSAF

301 AIHDMEEDTW YAAGILSFDK TCAVAEYGVY VRATDLKDWV QETMAKN

Show predicted peptides also

\section{Sort Peptides By}

(9) Residue Number

Obse rved
920.5013
2127.2664
1320.8011
972.5767
1817.0505
1833.0441
980.5405
1278.6979
1635.8950

\section{$\operatorname{Mr}(\operatorname{expt})$} 919. 4940 2126.2591

1319.7938

971.5694

1816.0432

1832.0368

979.5333

1277.6906

1634.8877
Increasing Mass

Decreasing Mass

ppm
42
57
46
42
58
57
47
45
55

\section{Miss Sequence}

0 K. GSFPWQAK.M (Ions score 32)

0 R. HGLTTGATLISDQWLLTTAK.N (Ions score

0 K. DITPTLTLYVGK.N (Ions score 23)

o K. NQLVEIEK.V (Ions score 24)

1 R. VMPICLPSKDYIAPGR.V Carbamidomethyl

1 R. VMPICLPSKDYIAPGR.V Carbamidomethy 1

0 R.VGYVSGWGR.N (IOnS score 39)

0 K. YVMLPVADQDK. C (Ions score 37 )

1 R. ATDLKDWVQETMAK. N (Ions score 26) 
Sample M7

4700 Reflector Spec \#1 MC=>NR(2.00)[BP = 1060.6, 10705]

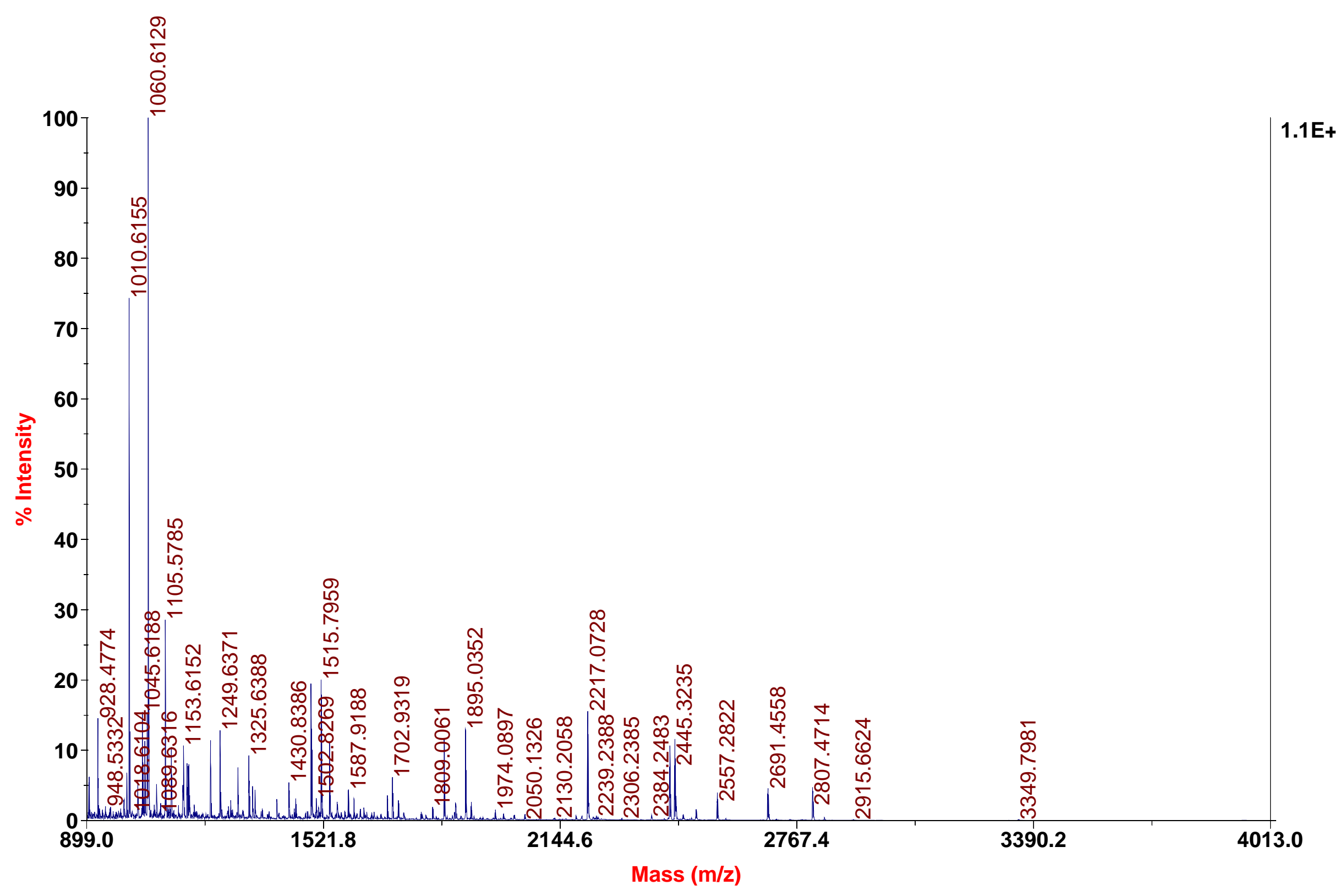






Concise Protein Summary Report
Format As
Concise Protein Summary
$\underline{\text { Help }}$
Significance threshold $p<0.05$
Max. number of hits AUTO

Re-Search All

Search Unmatched

1. Q32F18 MoUSE Mass: 223203 Score: 120 Expect: 9.2e-08 Matches: 95 Myosin, heavy polypeptide 1, skeletal muscle, adult.-Mus musculus (Mouse). Q9ESW6 MOUSE Mass: 54545 Score: 40 Expect: 9.2 Matches: 31 Myosin heavy chain IIX (Fragnent). - Mus musculus (Mouse). 




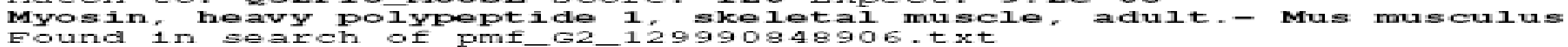

1 10013

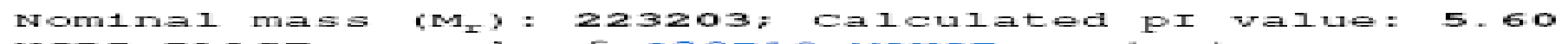

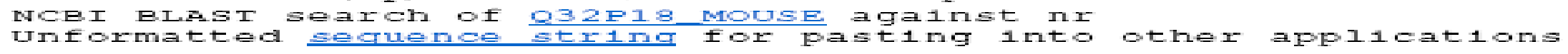

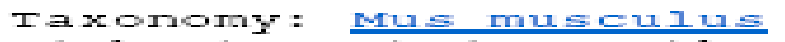

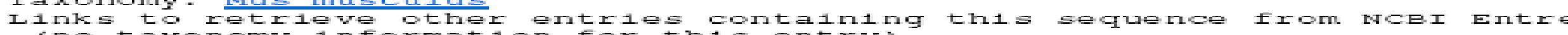

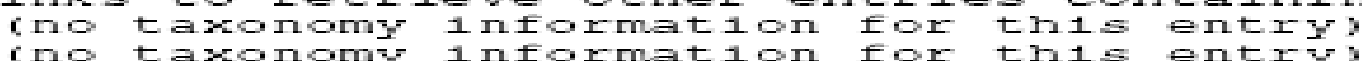

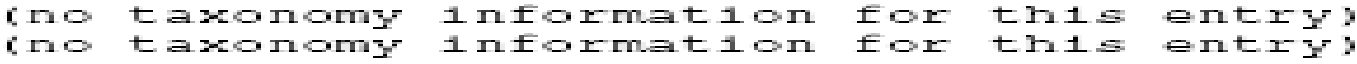

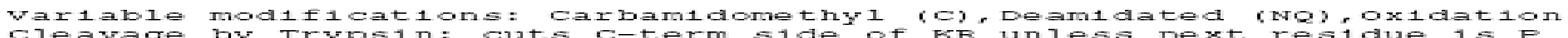

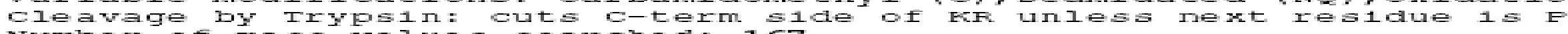

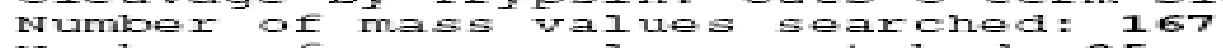

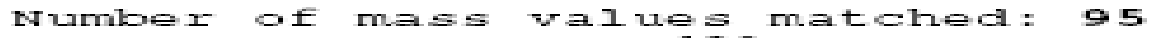





1 MSSDA EMA VE 51 TWOSFECOR: 101 DWIYITIEFE ISI I IEFIIFEI 201 TIGEFIIIEFI 201 TIAEFIFEFI $\begin{array}{ll}251 & \text { IFIIFGIIGI } \\ 301 & \text { IEI-ILIIIIII }\end{array}$ 351 SI YIIIGIMT 401 AICYPREFUC 451 DTIOOFOYFI

501 OEEYFIFGIF

551 FECNIIYESHI 601 HDPINETUVC $651 \quad E O T=B I T F E$ 701 FECNGWIECII 751 IIILSIDIDI BOI GXIAFUEYQK B51 ETEIEEIIIIIII 901 FADSI.ADAE 951 EDECEEIERD IOOI IEEIIAIOEAF

1051 IFIDIEFMIEF

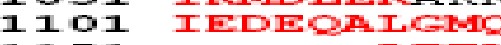
I151 EEISEFIEEI 1201 IERHADSUAEI

I251 IUIFIMMCRTE 그므 FDSIN 1351 FEQYEEEQEA ᄀ401 IOFLODAEEF 1451 FEOFITEFII I501 HIEIIIFENI I 551 IEEIEISLEH

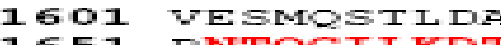
1651 1701 QIEFS FIIAF ᄀ75I IVOFARADEF

Iอ51 EFFEFIITO

1901 IFFFFIOHEI
CEAMP IIFES IIETEGSTI: IENIII TIY NASOFII TDF ISG IENOCTIF $L I S Z D I E I Y I$ YDYAF HIGINIEF ICOI NEYYTECOTH GIIIIGEEI MEETIEGMI GFSNMUERER I Yoks SI-IIK I WLIIIIITIII I CFICGE TOYKEGH'TEMVEFESIEC EEEEIIIDENL RCDOIIFTFI IDDIEITIIR POTIDDIOAF ILLEDIILIS LOFK I FEIOI CCATSAOIEN GEOIDNIDR: DQV SEIRTEE CFOAF TOOIF IAEIPRAMSI
VRA VARCAS VFA VAAKCAS ㅋNIOOKYEET NIQDEISDIT FIFEFWDIIF OIFT 2IT $T$ 工 CEIDASER


ILFOGFICIO
IEEDFEDVIL EFAERREDII
EIRERTEMOTR I FICDOWTEN-1 IFCOI" ENOSIIITGE DOIISRTPII IEISEUTEOI IIVE IDIOE QFEEOEFEC QOW YNS UCA I EDIEISIDI:


PIFCF

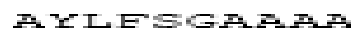
STIEHE MECI IXA DIFSRTE EFIIGIIGII IOYMFFAFMT

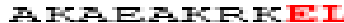
-TIT工TET CICE UE EDIEUAITEI EEIMDEEIDE FIEEIEEEIE FOFIFIEFEE EEOORIINET -T ANSENAPWFT IEKTIRIDRT HIRIEI SOFE EOIAECOKFI EIIUQVISEI IIIFEATEDIN QEDIKE OI.A DIIHTONTSI MITAEEIFKES IOLIFDILIS $E \equiv 0$ WIII IFWE
EFDIFESTH ITEFATDIEC NIFWTEIRE GOZGFT YNTI FITCMITIVE FAEFSYHIFY EII-IATISII D TEVADIIAAXI AFEYTEFIEI INF TIEFIOC MOIE SIIEFE

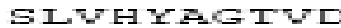
IEIE $=$ GGOS IFNETILTCA VINA SAIEEC EEI-IFLIII.A. VESHWEWMIEL EE GMUA IMOEE EFIEDEEEII FWRIITEER-TS FCIIIEOOUDD QCI DEISI EIIE IERISFIREE MFIDIEEATI MIE-IEIDDIES TAOFCRIOTE FIIISIITHAT KXETDAIDRT EVEDIIIIDVE SRSISTELFE HEIERIFEROI FEIAERDEEI EMEIDIIT $S \mathrm{~N}$ YEFFATI TER INTFERIETI DTERHIEFITE GEVEMEOFE FIII TIROAE SFEUHIFIIS
YDAIESFUEA MIIMIT HI HEF IIXFOEIFOE FWIOYEIIII NDNSSEFGEF QIMSNIEIEDI. IIGFI SIEF\% QNIINADIIE

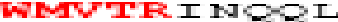
FENHAMEW IE CWIEFEITDIS YNII FERIAREIGSS MEFEIVIFOI QEIDSERASE IITFTOAMCF FEIIIEILISII FIIDIOLOUDS IEIIIESIREL GLDETIAKIT IECSIEDEFE EEEMSMIPSK FCFS DISFEI QHEATAITIF INWIFTI SIRSEG SCEYSROIDE OSSFHDCDI. EEIEEAIERTL FTMARCARID IFUI ESEKEEI0II DOLFEIIIIE:FEMAIEALIFI EIEEIRATIE ISQISCEMED FEIIEOTWFDI. YEIIFGIREI

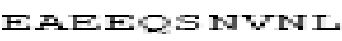
EE 


\section{M7}

4700 MS/MS Precursor 928.478 Spec \#1 MC[BP = 110.1, 1013]

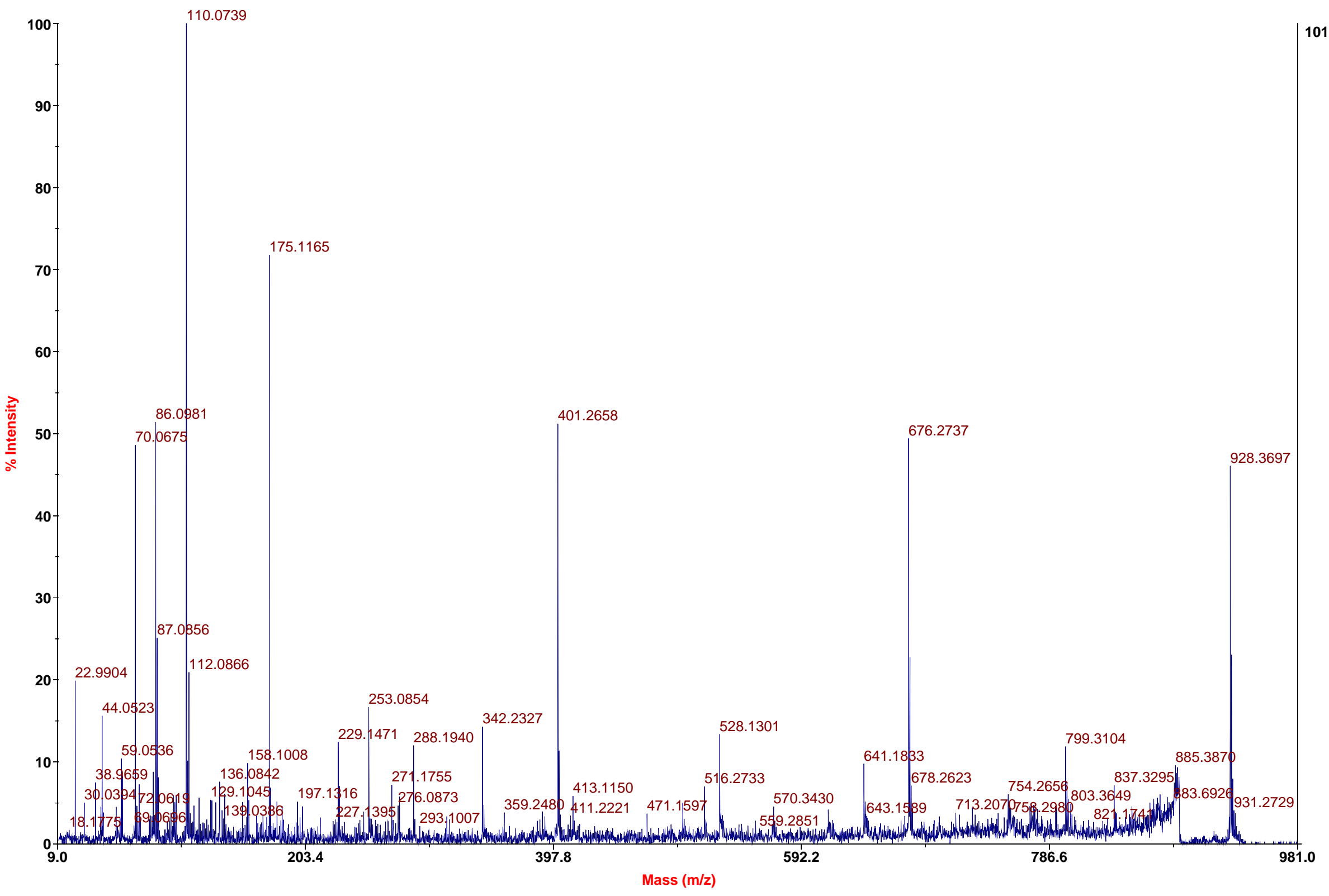




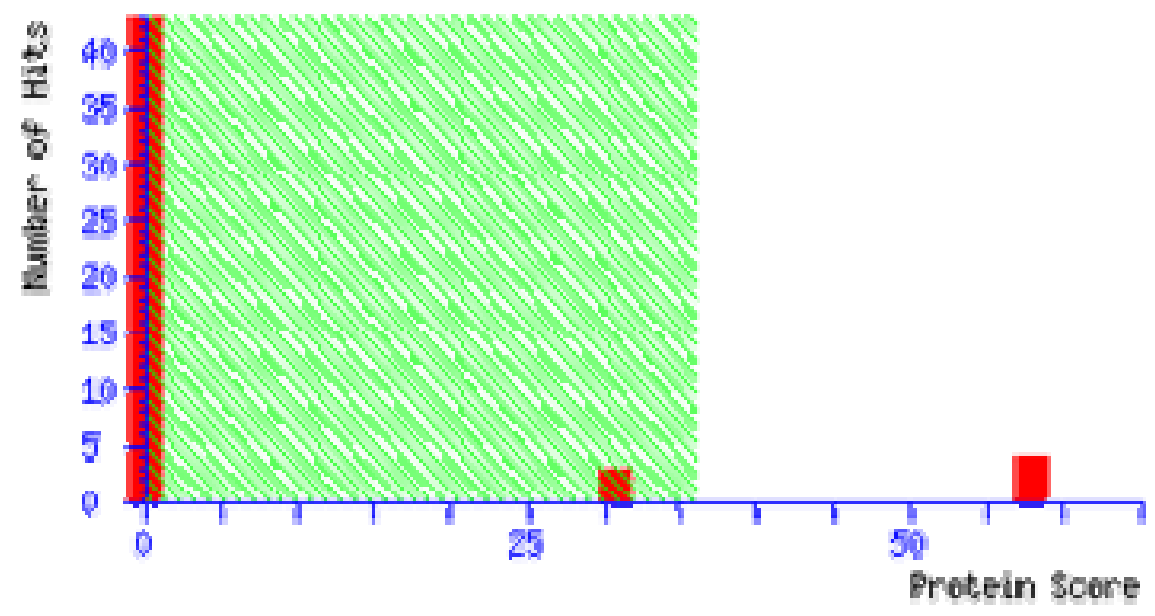

\section{Peptide Summary Report}

Significance threshold $p<\longdiv { 0 . 0 5 }$ Max. number of hits AUTO

Standard scoring $\bigcirc$ MudPIT scoring Ions score or expect cut-off 0

Show sub-sets 0

Show pop-ups Suppress pop-ups Sort unassigned Decreasing Score - Require bold red

\section{Select All \\ Select None \\ Search Selected \\ Error tolerant}

1. Q9JHR4 MOUSE Mass: 60994 Score: 58 Matches: 1(1) Sequences: 1(1) emPAI: 0.10 Myosin heavy cha1n IIB (Fragnent).- Mus musculus (Mouse).

$\square$ check to include this hit in error tolerant search

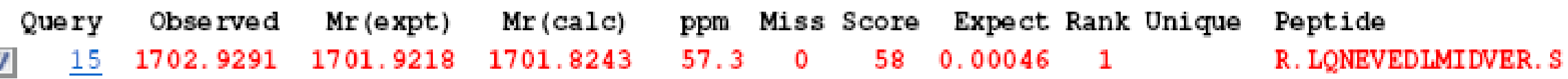




\section{Protein View}

Match to: Q9JHR4_MOUSE score: 58

Myosin heavy chain IIB (Fragment).- Mus musculus (Mouse).

Found 1n search of PPi__G2_129990854806.txt

Nomlnal mass $\left(\mathrm{M}_{\mathrm{r}}\right)$ : 60994 ; Calculated $\mathrm{pI}$ value: 5.38

NCBI BLAST search of Q9JHR4_MOUSE against $n r$

unformatted sequence string for pasting into other applications

Taxonony: Mus musculus

Links to retrleve other entries contalning this sequence from. NCBI Entrez:

(no taxonomy information for this entry)

Varlable modif1cations: Carbanidonethyl (C), Deanidated (NQ), oxidation (M) cleavage by Trypsin: cuts c-term. side of KR unless next residue is $\mathrm{F}$ sequence coverage: 2 :

Matched peptides shown 1n Bold Red

1 SLEKT KQRLQ NEVEDLMIDV ERSNAACAAL DKKORNFDKV LAEMKQKYEE

51 TQAELEASQK ESRSLSTELF KVKNAYEESL DQLETLKREN KNLQOEISDL

101 TEQIAEGGKH IHELEKIKKQ IDQEKSELQA SLEEAEASLE HEEGKILRIQ

151 LELNOVKSEI DRKIAEKDEE IDQLKRNHLR VVESMQSTLD AEIRSRNDAL

201 RI KKKMEGDL NEMEIQLNHA NRQAAEAIRN LRNTOGMLRD TQLHLDDALR

251 GQDDL KEQLA MVERRANLMQ AEIEELRASL EQTERSRRVA EQELLDASER

301 VQLLHTQNTS LINTKKKLET DISQIQGEME DIVOEARNAE EKAKKAITDA

351 AMMAEELKKE QDTSAHLERM KKMMEQTVKD LQHRLDEAEQ LALKGGKKQI

401 QKLEARVREL ENEVENEQKR NIEAVKGLRK HERRVKELTY QTEEDRKNVL

451 RLQDLVDKLQ TKVKAYKRQA EEAEEQSNVN LAKFRKIQHE LEEAEERADI

501 AESQVNKLRV KSREVHTKVI SEE 


\section{Sample M8}

4700 Reflector Spec $\# 1 \mathrm{MC}=>\mathrm{NR}(2.00)[\mathrm{BP}=1558.0,16431]$

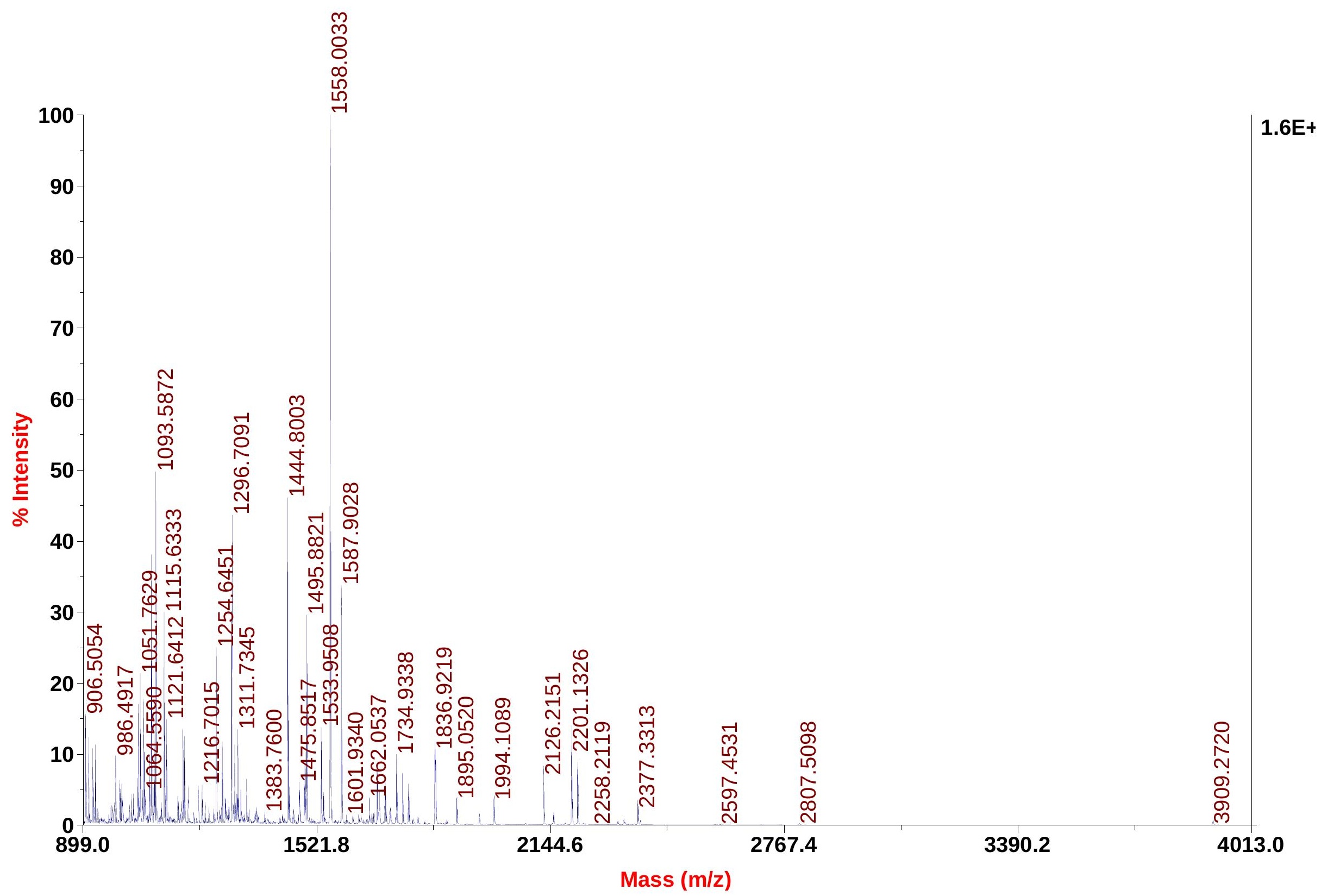




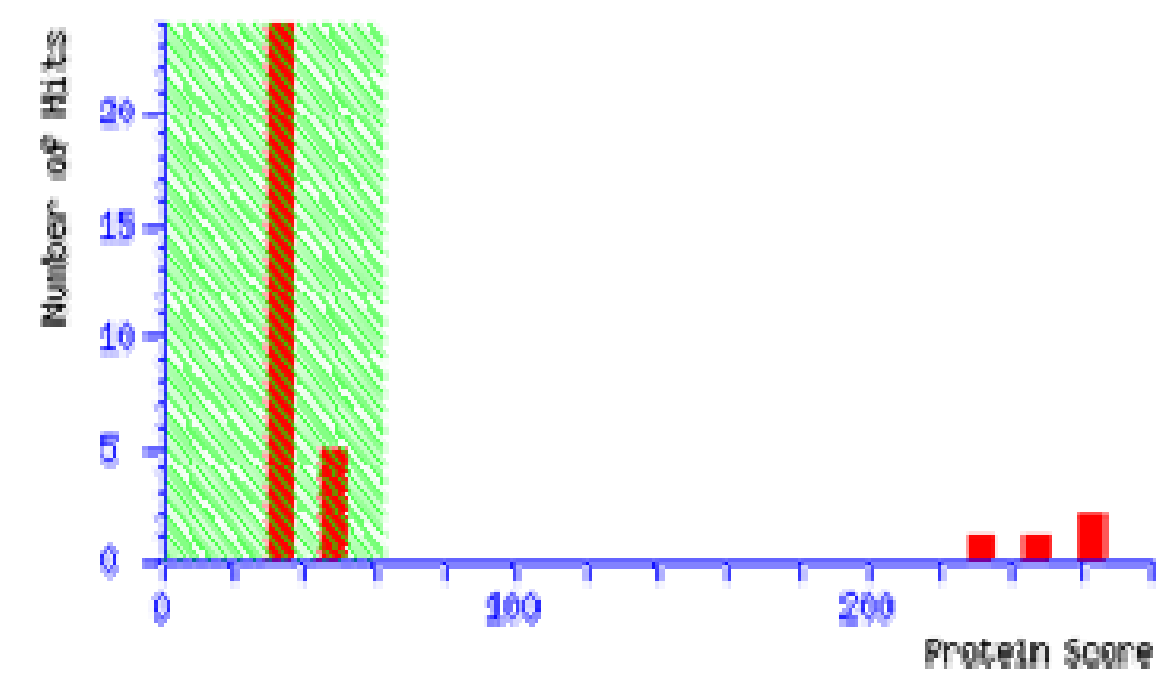

\section{Concise Protein Summary Report}
Format As
Concise Protein Summary
$\underline{\text { Help }}$
Significance threshold $p<0.05 \quad$ Max. number of hits AUTO

\section{Re-Search All}

Search Unmatched

1. CAA69019 Mass: 51533 Score: 262 Expect: $5.8 e-22$ Matches: 65

MMVIMENT NID: - Mus musculus

Q3UD36 MOUSE Mass: 47148 Score: 213 Expect: $4.6 \mathrm{e}-17$ Matches: 61

Bone marrow macrophage CDNA, RIKEN full-length enriched l1brary, clone: I830001B16 product:vinentin, BAC28181 Mass: 17978 Score: 69 Expect: 0.011 Matches: 25

AK033175 NID: - Mus musculus 


\section{Protein View}

Match to: CAA69019 score: 262 Expect: 5.8e-22 MMVIMENT NID: - Mus musculus

Found 1n search of pmf_H2_129990848907.txt

Nomlnal mass $\left(M_{x}\right)$ : 51533; Calculated pI value: 4.96

NCBI BLAST search of CAA69019 aga1nst nr

unformatted sequence string for pasting 1nto other applications

Taxonony: Mus musculus

Var1able mod1f1cat1ons: Carban1domethyl (C), Dean1dated (NQ), ox1dat1on (M) cleavage by Trypsin: cuts c-term side of KR unless next residue $15 \mathrm{~F}$

Number of mass values searched: 162

Number of mass values matched: 65

sequence coverage: 898

Matched peptides shown in Bold Red

1 SSRPSSNRSY VTTSTRTYSL GSALRPSTSR SLYSSSPGGA YVTRSSAVRL

51 RSSVPGVRLL QDSVDFSLAD AINTEFKNTR TNEKVELQEL NDRFANYIDK

101 VRFLEQQNKI LLAELEQLKG QGKSRLGDLY EEEMRELRRQ VDQLTNDKAR

151 VEVERDNLAE DIMRLREKLQ EEMLQREEAE STLQSFRQDV DNASLARLDL

201 ERKVESLQEE IAFLKKLHDE EIQELQAQIQ EQHVQIDVDV SKPDLTAALR

251 DVRQQYESVA AKNLQEAEEW YKSKFADLSE AANRNNDALR QAKQESNEYR

301 RQVQSLTCEV DALKGTNESL ERQMREMEEN FALEAANYQD TIGRLQDEIQ

351 NMKEEMARHL REYQDLLNVK MALDIEIATY RKLLEGEESR ISLPLPTFSS

401 LNLRETNLES LPLVDTHSKR TLLIKTVETR DGQVINETSQ HHDDLE

Show predicted peptides also

\section{Sort Peptides By}

$\begin{aligned} \text { Start } & - \text { End } \\ 9 & -16 \\ 17 & -30 \\ 31 & -44 \\ 59 & -77 \\ 81 & -93 \\ 85 & -93 \\ 94 & -102 \\ 101 & -109 \\ 103 & -109 \\ 103 & -109 \\ 110 & -119 \\ 110 & -123\end{aligned}$

- Residue Number

Observed
914.5060
1495.8798
1444.7979
2126.2109
1587.9003
1115.6313
1125.6686
1162.6250
906.5054
908.4515
1169.7764
1540.0065

\section{Mr (expt)}

913.4988

1494.8725

1443.7906

2125.2036

1586.8930

1114.6240

1124.6613

1161.6177

905. 4981

907.4443

1168.7691

1538.9992
Increasing Mass

Decreasing Mass

$M r(c a l c)$
913.4505
1494.7790
1443.6994
2125.0579
1586.7900
1114.5618
1124.5978
1161.6142
905.4607
907.4287
1168.7067
1538.9032

ppm
53
63
63
69
65
56
56
3
41
17
53
62

\section{Miss Sequence}

R. SYVTTSTR. T

R. TYSLGSALRPSTSR. S

R. SLYSSSPGGAYVTR. S

R. LLQDSVDF SLADAINTEFK . N

R. TNEKVELQELNDR . F

K. VELQELNDR . F

R. FANYIDKVR . F

K. VRF LEQQNK . I

R. FLEQQNK. I

R. FLEQQNK. I 2 Deamidated (NQ)

K. ILLAELEQLK. G

1 K. ILLAELEQLKGQGK. S 


\section{M8}

4700 MS/MS Precursor 1051.76 Spec \#1 MC[BP = 102.1, 2153]






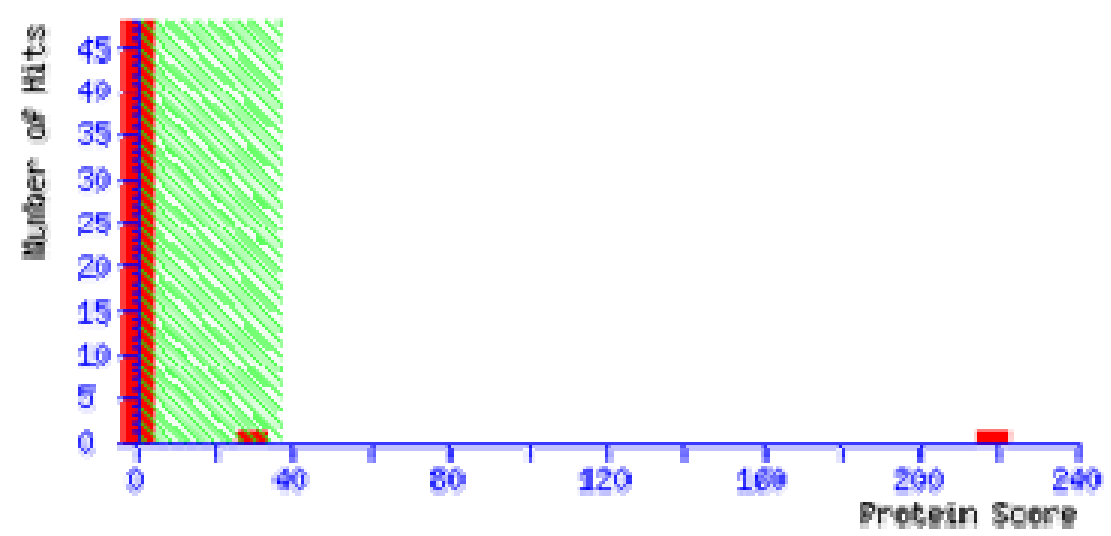

\section{Peptide Summary Report}

Format As Peptide Summary

Significance threshold $\mathrm{p}<0.05$

Standard scoring $\odot$ MudPIT scoring $\odot$ Ions score or expect cut-off 0

Show pop-ups Suppress pop-ups Sort unassigned Decreasing Score
Help

Show sub-sets 0

Require bold red

\section{Select All}

Select None

Search Selected

\section{Error tolerant}

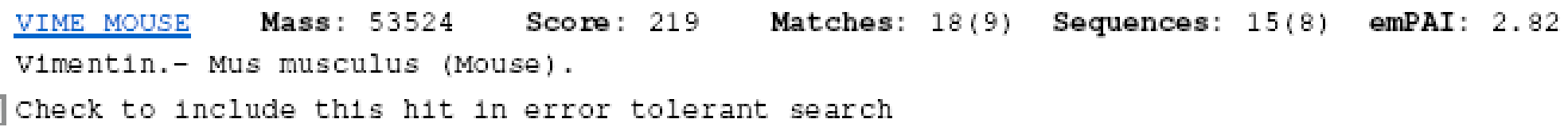

\begin{tabular}{|c|c|c|c|c|c|c|}
\hline Query & rved & $\mathrm{xpt}$ ) & $\mathrm{Mr}$ & pm & Miss & . \\
\hline$\underline{1}$ & 914.5060 & 913.4988 & 913.4505 & 2.8 & 0 & 23 \\
\hline$=$ & 1081.5625 & 1080.5552 & 1080.4948 & 55.9 & 1 & 17 \\
\hline & 1093.5869 & 092.5796 & 109 & 4. & & 8 \\
\hline & 1115 & 0 & $11:$ & 55. & & 1 \\
\hline & 1254.6449 & 253.6376 & 1253.5598 & 2 . & 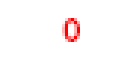 & 38 \\
\hline & 1270.6376 & 269.6303 & 1269.5547 & . & & (12) \\
\hline & 1295.7449 & 1294.7376 & 1294.6591 & 60.7 & 0 & 31 \\
\hline
\end{tabular}

Expect Rank Unique

$\begin{array}{rrr}1.2 & 1 & \mathrm{U} \\ 4.9 & 1 & \mathrm{U} \\ 0.005 & 1 & \mathrm{U} \\ 0.2 & 1 & \mathrm{U} \\ 0.044 & 1 & \mathrm{U} \\ 21 & 1 & \mathrm{U} \\ 0.16 & 1 & \mathrm{U}\end{array}$

Peptide

R. SYVTTSTR. T

K. QESNEYRR. $Q$

K. FADLSEAANR . N

K. VELQELNDR. F

R. LGDLYEEEMR. E

R. LGDLYEEEMR. E + Oxidation

K. MALDIEIATYR. $K$ 


\section{Protein View}

Match to: VIME_MOUSE Score: 219

Vimentin.- Mus musculus (Mouse).

Found 1n search of ppw_H2_129990854907.txt

Nom1nal mass $\left(\mathrm{M}_{\mathrm{r}}\right)$ : 53524; Calculated pI value: 5.06

NCBI BLAST search of VIME_MOUSE aga1nst nr

unformatted sequence string for pasting 1nto other applications

Taxonony: Mus musculus

Var1able mod1f1cations: Carbanidonethyl (C), Dean1dated (NQ), ox1dation (M)

cleavage by Trypsin: cuts C-term side of $\mathrm{KR}$ unless next residue $1 \mathrm{~s} P$

sequence Coverage: 38 \%

Matched peptides shown 1n Bold Red

1 STRSVSSSSY RRMFGGSGTS SRPSSNRSYV TTSTRTYSLG SALRPSTSRS

51 LYSSSPGGAY VTRSSAVRLR SSVPGVRLLQ DSVDFSLADA INTEFKNTRT

101 NEKVELQELN DRFANYIDKV RFLEQQNKIL LAELEQLKGQ GKSRLGDLYE

151 EEMRELRRQV DQLTNDKARV EVERDNLAED IMRLREKLQE EMLQREEAES

201 TLQSFRQDVD NASLARLDLE RKVESLQEEI AFLKKLHDEE IQELQAQIQE

251 QHVQIDVDVS KPDLTAALRD VRQQYESVAA KNLQEAEEWY KSKFADLSEA

301 ANRNNDALRQ AKQESNEYRR QVQSLTCEVD ALKGTNESLE RQMREMEENF

351 ALEAANYQDT IGRLQDEIQN MKEEMARHLR EYQDLLNVKM ALDIEIATYR

401 KLLEGEESRI SLPLPTFSSL NLRETNLESL PLVDTHSKRT LLIKTVETRD

451 GQVINETSQH HDDLE

Show predicted peptides also

\section{Sort Peptides By Residue Number Increasing Mass Decreasing Mass}

$\begin{array}{rrrrr}\text { Start - End } & \text { Observed } & \text { Mr(expt) } & \text { Mr(calc) } & \text { ppm } \\ 28-35 & 914.5060 & 913.4988 & 913.4505 & 53 \\ 36-49 & 1495.8798 & 1494.8725 & 1494.7790 & 63 \\ 50-63 & 1444.7979 & 1443.7906 & 1443.6994 & 63 \\ 100-112 & 1587.9003 & 1586.8930 & 1586.7900 & 65 \\ 104-112 & 1115.6313 & 1114.6240 & 1114.5618 & 56 \\ 145-154 & 1254.6449 & 1253.6376 & 1253.5598 & 62 \\ 145-154 & 1270.6376 & 1269.6303 & 1269.5547 & 60 \\ 222-234 & 1533.9507 & 1532.9434 & 1532.8450 & 64\end{array}$

\section{Miss Sequence}

0 R. SYVTTSTR. T (Ions score 23)

0 R. TYSLGSALRPSTSR.S (Ions score 9)

0 R. SLYSSSPGGAYVTR.S (Ions score 24)

1 R. TNEKVELQELNDR. F (IONS score 47)

0 K. VELQELNDR.F (IOnS score 31)

0 R. LGDLYEEEMR. E (IOns score 38)

0 R. LGDLYEEEMR. E ox1dation (M) (Ions score 12)

$222-234$

1533.9507

1 R. KVESLQEEIAFLK. K (IOns score 40) 
Sample M9

4700 Reflector Spec \#1 MC=>NR(2.00)[BP = 1558.0, 22567]

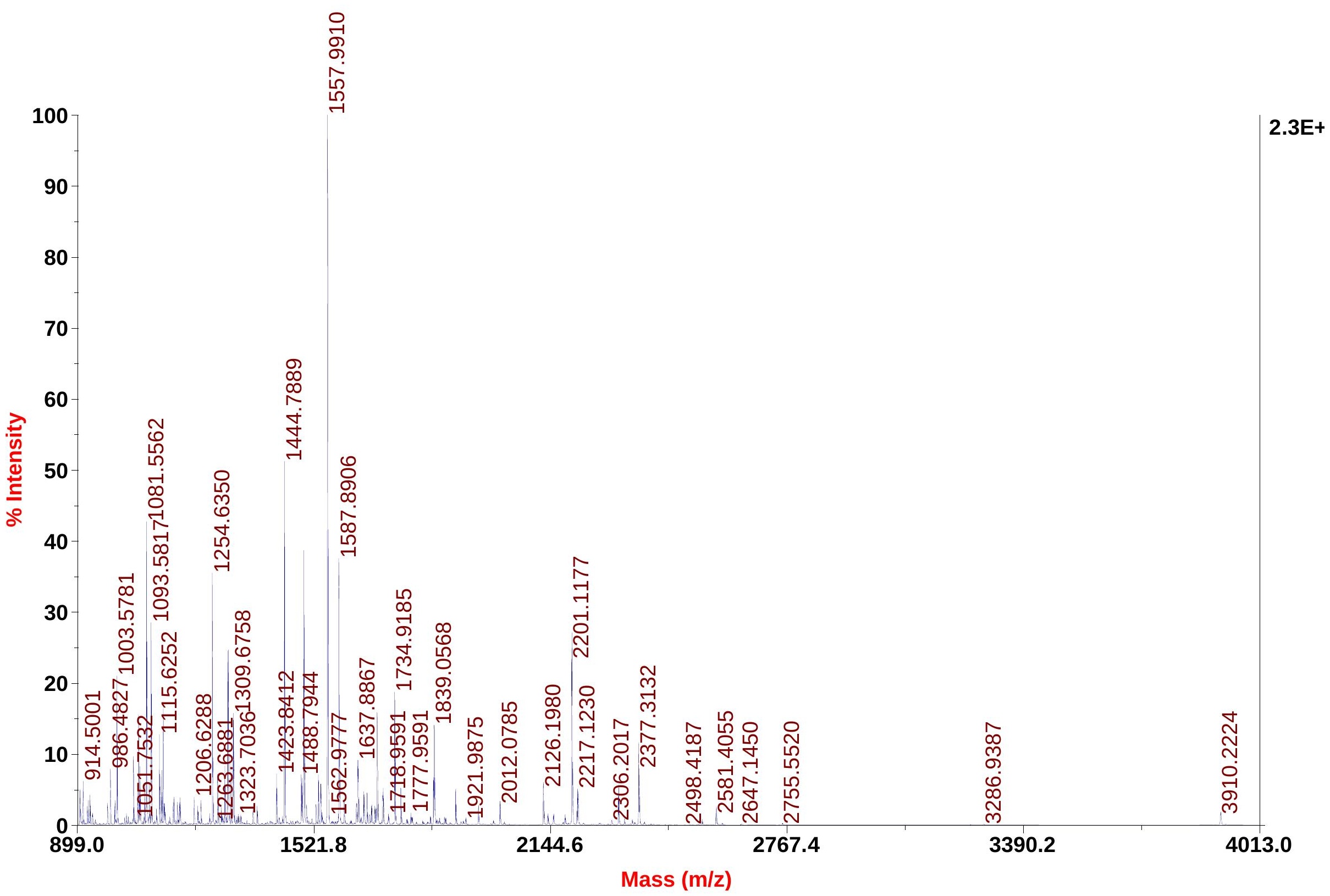




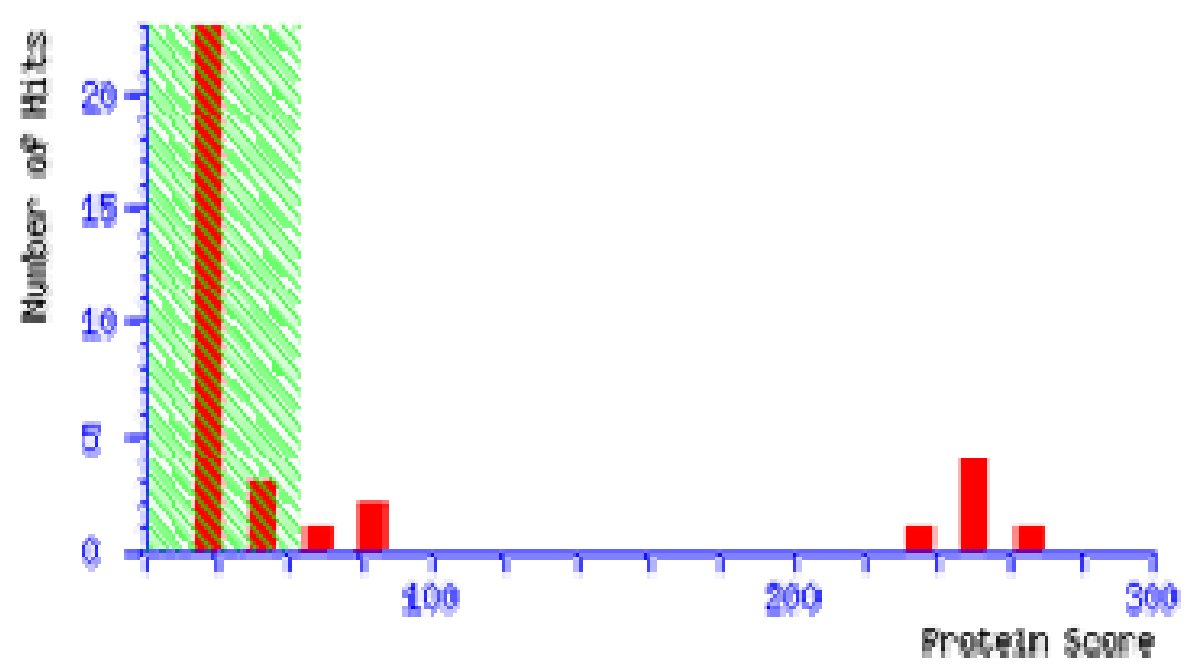

\section{Concise Protein Summary Report}

Format As Concise Protein Summary .

Significance threshold $p<\overline{0.05}$ Max. number of hits AUTO

\section{Re-Search All Search Unmatched}

1. Q5FWJ3 MOUSE Mass: 53655 Score: 265 Expect: $2.9 e-22$ Matches: 77

V1mentin (NOD-derived CD11c +ve dendrit1c cells cDNA, RIKEN full- length enriched library, clone: VIME MOUSE Mass: 53524 Score: 265 Expect: $2.9 \mathrm{e}-22$ Matches: 77

V1ment1n.- Mus musculus (Mouse).

CAR69019 Mass: 51533 Score: 258 Expect: $1.5 e-21$ Matches: 75

MMVIMENT NID: - Mus musculus

Q3TFD9_MOUSE Mass: 53656 Score: 253 Expect: 4.6e-21 Matches: 75

17 days embryo heart cDNA, RIKEN full-length enr1ched l1brary, clone:I920087013 product:vimentin, 


\section{Protein View}

Match to: Q5FWJ3_MOUSE score: 265 Expect: $2.9 e-22$

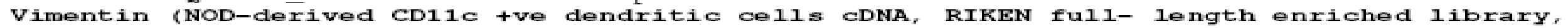
Found $1 \mathrm{n}$ search of pmf_I2_129990848908.txt

Nom1nal mass $\left(M_{I}\right): 53655$; calculated pI value: 5.06 NCBI BLAST search of Q5EWJ3 MOUSE aga1nst nr unformatted sequence string for pasting into other applications

Taxonorny: Mus musculus

Links to retrieve other entries contalning this sequence from NcBI Entrez: (no taxonomy 1 nformation for this entry)

(no taxonomy 1 nformation for this entry)

(no taxonomy information for this entry)

(no taxonomy information for this entry)

Var1able modif1cations: Carbam1domethyl (c), Deamidated (No), oxidation cleavage by Trypsin: cuts c-term side of KR unless next residue $1 s$ p Number of mass values searched: 181

Number of mass values matched: 77

sequence coverage: 898

Matched peptides shown 1n Bold Red

1 MSTRSVSSSS YRRMEGGSGT SSRPSSNRSY VTTSTRTYSL GSALRPSTSR

51 SLYSSSPGGA YVTRSSAVRL RSSVPGVRLL ODSVDFSIAD AINTEEKNTR

101 TNEKVELQEL NDRFANYIDK VRFLEQQNKI LLAELEQLKG QGKSRLGDLY

151 EEEMRELRRQ VDQLTNDKAR VEVERDNIAE DIMRLREKLQ EEMLQREEAE

201 STLOSFRQDV DINASLARLDL ERKVESLQEE IAFLKKLHDE EIQELQAQIO

251 EQHVQIDVDV SKPDLTAALR DVRQQYESVA AKNLQEAEEW YKSKFADLSE

301 AANRNNDALR QAKQESNEYR RQVQSLTCEV DALKGTMESL ERQMREMEEN

351 FALEAANYOD TIGRIODEIO NMKEFMARHL REYODILNVK MALDIEIATY

401 RKLLEGEESR ISLPLPTFSS LNLRETNLES LPLVDTHSKR TLLIKTVETR

451 DGQVINETSQ HHDDLE

Show predicted peptides also

\section{Sort Peptides By}

Start - End
$5-13$
$14-28$
$29-36$
$29-50$
$37-50$
$51-64$
$79-97$
$79-100$
$101-113$
$105-113$
$114-122$
$121-129$

O Residue Number

Observed
1028.5667
1543.7866
914.5001
2391.3230
1495.8713
1444.7861
2126.1934
2498.3972
1587.8877
1115.6230
1125.6603
1162.6176

Mr (expt)
1027.5594
1542.7793
913.4928
2390.3157
1494.8640
1443.7788
2125.1861
2497.3899
1586.8804
1114.6157
1124.6530
1161.6103

Increasing Mass
Decreasing Mass

$\begin{array}{rr}\text { Mr (calc) } & \text { ppm } \\ 1027.5047 & 53 \\ 1542.6845 & 61 \\ 913.4505 & 46 \\ 2390.2190 & 40 \\ 1494.7790 & 57 \\ 1443.6994 & 55 \\ 2125.0579 & 60 \\ 2497.2336 & 63 \\ 1586.7900 & 57 \\ 1114.5618 & 48 \\ 1124.5978 & 49 \\ 1161.6142 & -3\end{array}$

\section{Miss Sequence}

1 R. SVSSSSYRR. M

O R. MIGGSGTSSRP

- R. SYVTTSTR. T

O

o

0

1

1

0

1

K. VELQELNDR . F

R. FANYIDKVR , F

R SYVTTSTRTYSIGSALRPSTSR

R. TYSLGSALRPSTSR, S

R. SLYSSSPGGAYVTR. S

R. LLQDSVDFS LADAINTEFK . N

R. LLQDSVDFSIAADAINTEFKNTR

R. TNEKVELQELNDR - F

K. VRF LEQQNK . I

Deamidate 


\section{M9}

4700 MS/MS Precursor 1557.99 Spec \#1 MC[BP = 86.1, 11204]

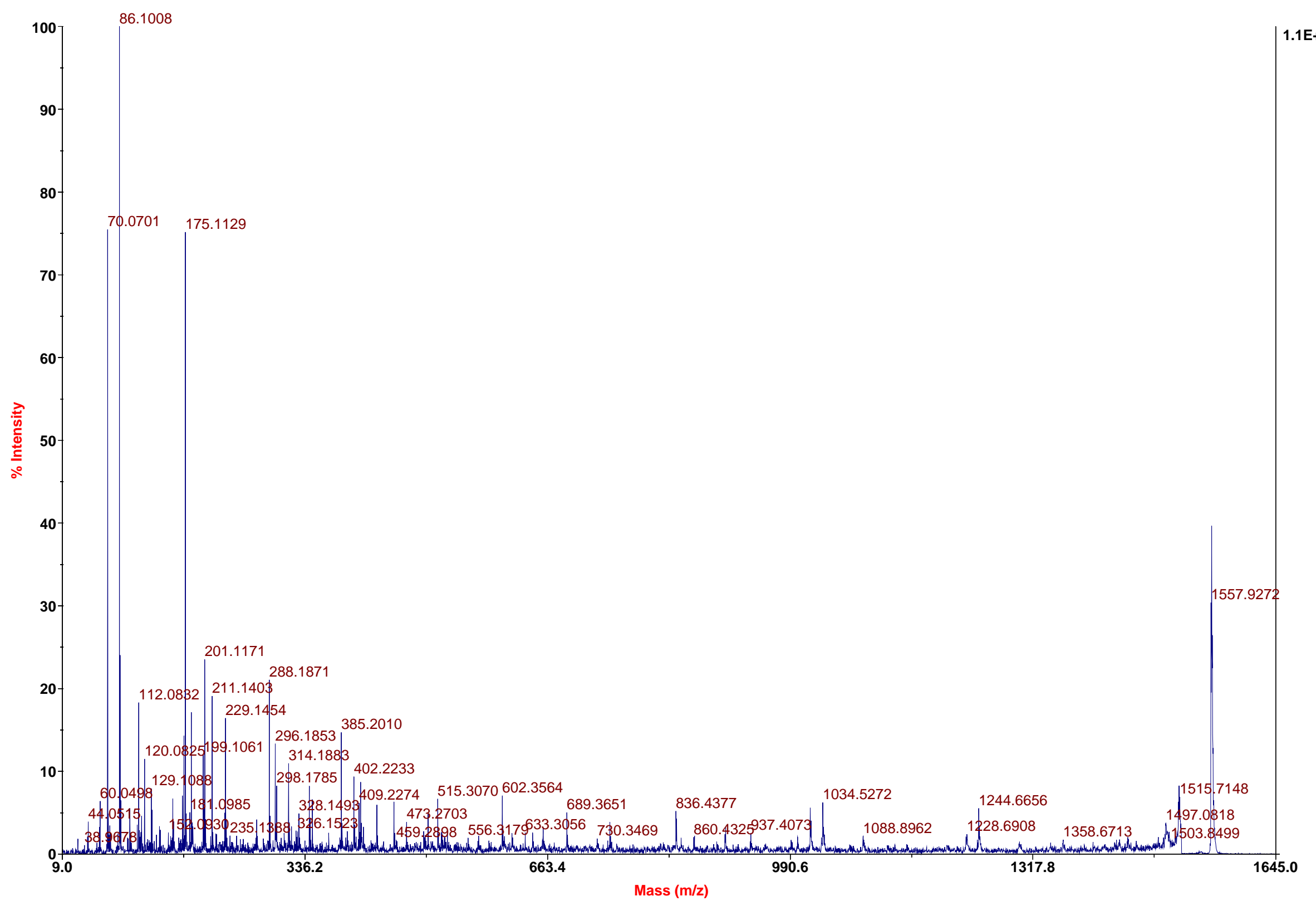




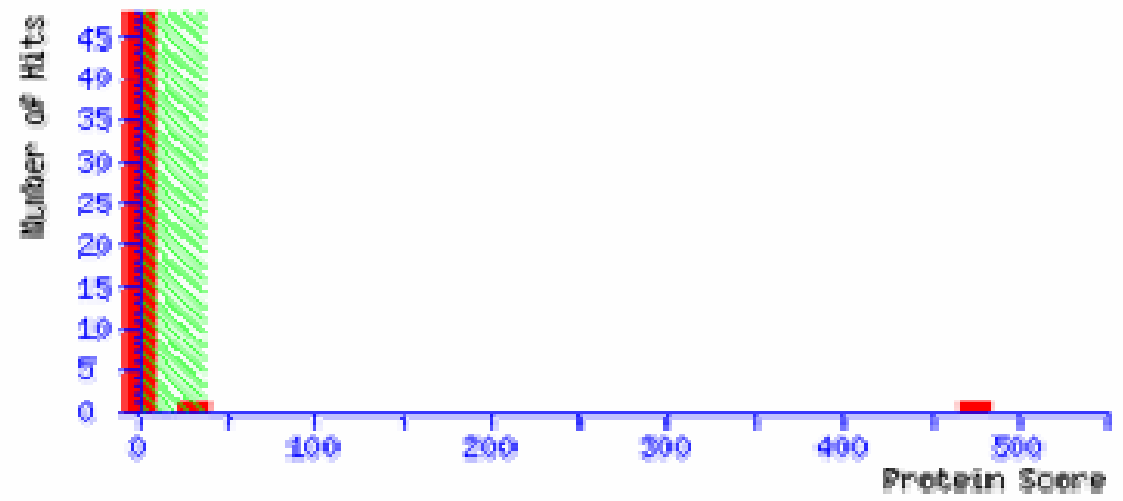

\section{Peptide Summary Report}

\section{Format As Peptide Summary}

Significance threshold $p<0.05$

Standard scoring $\bigcirc$ MudPIT scoring Ions score or expect cut-off 0

Show pop-ups Suppress pop-ups Sort unassigned Decreasing Score
Help

Show sub-sets 0

Require bold red

\section{Select All}

\section{Select None}

Search Selected

\section{Error tolerant}

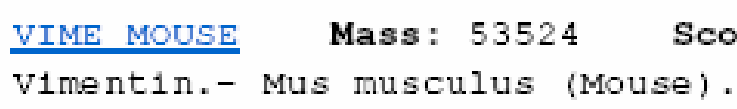

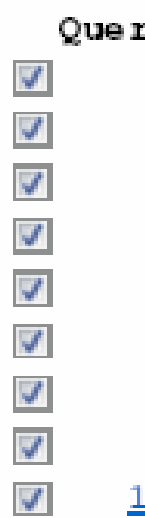

$\begin{array}{rrc}\text { Observed } & \text { Mr(expt) } & \text { Mr(calc) } \\ 1046.5822 & 1045.5749 & 1045.5226 \\ 1081.5541 & 1080.5468 & 1080.4948 \\ 1093.5795 & 1092.5722 & 1092.5200 \\ 1115.6230 & 1114.6157 & 1114.5618 \\ 1125.6603 & 1124.6530 & 1124.5978 \\ 1254.6342 & 1253.6269 & 1253.5598 \\ 1295.7336 & 1294.7263 & 1294.6591 \\ 1309.6732 & 1308.6659 & 1308.5986 \\ 1444.7861 & 1443.7788 & 1443.6994\end{array}$

$\begin{array}{lcc}\text { ppm } & \text { Miss } & \text { Score } \\ 50.0 & 0 & 24 \\ 48.2 & 1 & 27 \\ 47.8 & 0 & 61 \\ 48.4 & 0 & 31 \\ 49.1 & 1 & 35 \\ 53.6 & 0 & 71 \\ 51.9 & 0 & 44 \\ 51.5 & 0 & 46 \\ 55.0 & 0 & 60\end{array}$

\section{Ex}

\section{Expect Rank Unique}

1.2 (1)

0.53

0.00028

0.23

0.058

2. $3 e-05$

0.0081

0.0081

0.0003

\section{Peptide}

K. LQEEMLQR . E

K. QESNEYRR. $Q$

K. FADLSEAANR . N

K. VELQELNDR . F

R. FANYIDKVR . F

R. LGDLYEEEMR , E

K. MALDIEIATYR. $K$

K. NLQEAEEWYK . S

R. SLYSSSPGGAYVTR. S 


\section{Protein View}

Match to: VIME_MOUSE Score: $\mathbf{4 7 4}$

Vimentin. - Mus musculus (Mouse).

Found 1n search of Ppw_I2_129990855108.txt

Nom1nal mass $\left(M_{x}\right)$ : 53524; Calculated pI value: 5.06

NCBI BLAST search of VIME_MOUSE aga1nst nr

unformatted sequence string for pasting 1nto other applications

Taxonony: Mus musculus

Var1able modif1cations: Carban1domethy1 (C), Dean1dated (NQ), ox1dation (M)

cleavage by Trypsin: cuts C-term side of KR unless next residue is $P$

sequence coverage: $47 \%$

Matched peptides shown 1n Bold Red

1 STRSVSSSSY RRMFGGSGTS SRPSSNRSYV TTSTRTYSLG SALRPSTSRS

51 LYSSSPGGAY VTRSSAVRLR SSVPGVRLLQ DSVDFSLADA INTEFKNTRT

101 NEKVELQELN DRFANYIDKV RFLEQQNKIL LAELEQLKGQ GKSRLGDLYE

151 EEMRELRRQV DQLTNDKARV EVERDNLAED IMRLREKLQE EMLQREEAES

201 TLQSFRQDVD NASLARLDLE RKVESLQEEI AFLKKLHDEE IQELQAQIQE

251 QHVQIDVDVS KPDLTAALRD VRQQYESVAA KNLQEAEEWY KSKFADLSEA

301 ANRNNDALRQ AKQESNEYRR QVQSLTCEVD ALKGTNESLE RQMREMEENF

351 ALEAANYQDT IGRLQDEIQN MKEEMARHLR EYQDLLNVKM ALDIEIATYR

401 KLLEGEESRI SLPLPTFSSL NLRETNLESL PLVDTHSKRT LLIKTVETRD

451 GQVINETSQH HDDLE

Show predicted peptides also

\section{Sort Peptides By}

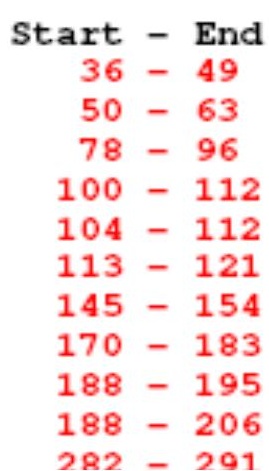

$\begin{array}{ccc}\text { Observed } & \text { Mr(expt) } & \text { Mr(calc) } \\ 1495.8713 & 1494.8640 & 1494.7790 \\ 1444.7861 & 1443.7788 & 1443.6994 \\ 2126.1934 & 2125.1861 & 2125.0579 \\ 1587.8877 & 1586.8804 & 1586.7900 \\ 1115.6230 & 1114.6157 & 1114.5618 \\ 1125.6603 & 1124.6530 & 1124.5978 \\ 1254.6342 & 1253.6269 & 1253.5598 \\ 1688.9269 & 1687.9196 & 1687.8199 \\ 1046.5822 & 1045.5749 & 1045.5226 \\ 2324.2590 & 2323.2517 & 2323.1114 \\ 1309.6732 & 1308.6659 & 1308.5986\end{array}$

Decreasing Mass
ppm Miss Sequence
$\begin{array}{llll}57 & 0 & \text { R. TYSLGSALRPSTSR.S (Ions score 8) }\end{array}$
550 R. SLYSSSPGGAYVTR.S (Ions score 60)
60
57
48
49
54
59
50
60
R. SLYSSSPGGAYTR. S (IONS score 60
R. LLQDSVDFSLADAINTEFK. N (Ions score 75)
1 R. TNEKVELQELNDR. F (IOnS score 71)
- K. VELQELNDR.F (IOnS score 31)
1 R. FANYIDKVR.F (IOnS score 35)
0 R. LGDLYEEEMR. E (IOnS score 71)
1 R. VEVERDNLAEDIMR. L (IOnS score 30)
O K. LQEEMLQR. E (I Ins score 24)
1 K. LQEEMLQREEAESTLQSFR. Q (IONS score 42)

51 
Sample M10

4700 Reflector Spec \#1 MC=>NR(2.00)[BP = 1558.0, 29698]

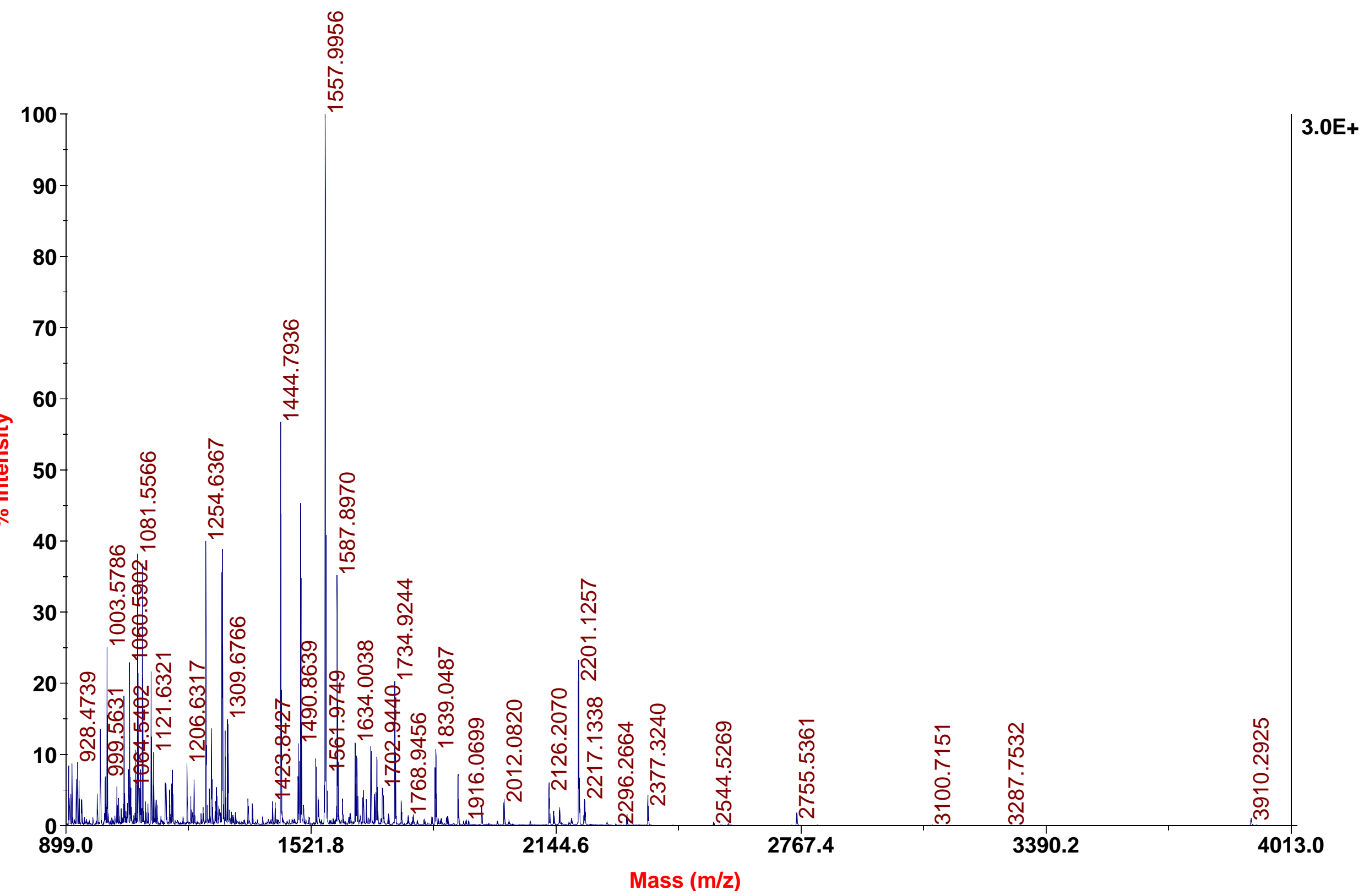






\section{Concise Protein Summary Report}

Format As Concise Protein Summary .

Significance threshold $p<0.05$ Max. number of hits AUTO

Re-Search All

Search Unmatched

1. CAA39807 Mass: 53689 Score: 217 Expect: $1.8 e-17$ Matches: 64 MMVMTM NID: - Mus musculus

2. VIME MOUSE Mass: 53524 Score: 216 Expect: $2.3 e-17$ Matches: 65

V1ment1n.- Mus musculus (Mouse).

Q5FWJ3_MOUSE Mass: 53655 Score: 216 Expect: 2.3e-17 Matches: 65

V1mentin (NOD-derived CD11c +ve dendritic cells cDNA, RIKEN full- length enriched l1brary, ( CAR69019 Mass: 51533 Score: 210 Expect: 9.2e-17 Matches: 64

MMVIMENT NID: - Mus musculus 


\section{Protein View}

Match to: CAA39807 score: 217 Expect: 1.8e-17

MMVMTM NID: - Mus musculus

Found 1n search of pmf_J2_129990849009.txt

Nomlnal mass $\left(M_{x}\right): 53689$; Calculated pI value: 5.06

NCBI BLAST search of CAA39807 aga1nst nr

unformatted sequence string for pasting 1nto other applications

Taxonorny: Mus musculus

Var1able mod1f1cat1ons: Carbanidomethyl (C), Deanidated (NQ), ox1dation

cleavage by Trypsin: cuts C-term side of KR unless next residue $15 \mathrm{~F}$

Number of mass values searched: 178

Number of mass values matched:

sequence coverage: 838

Matched peptides shown in Bold Red

1 MSTRSVSSSS YRRMFGGSGT SSRPSSNRSY VTTSTRTYSL GSALRPSTSR

51 SLYSSSPGGA YVTRSSAVRL RSSVPGVRLL QDSVDFSLAD AINTEFKNTR.

101 TNEKVELQEL NDRFANYIDK VRFLEQQNKI LLAELEQLKG QGKSRLGDLY

151 EEEMRELRRQ VDQFTNDKAR VEVERDNLAE DIMRLREKLQ EEMLQREEAE

201 STLQSFRQDV DNASLARLDL ERKVESLQEE IAFLKKLHDE EIQELQAQIQ

251 EQHVQIDVDV SKPDLTAALR DVRQQYESVA AKNLQEAEEW YKSKFADLSE

301 AANRNNDALR QAKQESNEYR RQVQSLTCEV DALKGTNESL ERQMREMEEN

351 FALEAANYQD TIGRLQDEIQ NMKEEMARHL REYQDLLNVK MALDIEIATY

401 RKLLEGEESR ISLPLPTFSS LNLRETNLES LPLVDTHSKR TLLIKTVETR.

451 DGQVINETSQ HHDDLE

\section{Show predicted peptides also}

\section{Sort Peptides By}

$\begin{aligned} \text { Start } & \text { End } \\ 2 & -12 \\ 5 & -13 \\ 29 & -36 \\ 29 & -50 \\ 37 & -50 \\ 51 & -64 \\ 79 & -97 \\ 101 & -113 \\ 105 & -113\end{aligned}$

(9) Residue Number

\section{Obse rved}

1216.6931

1028.5896

914.4987

2391.3303

1495.8760

1444.7904

2126.2061

1587.8940

1115.6248

$\begin{array}{rr}\text { Mr(expt) } & \text { Mr (calc) } \\ 1215.6858 & 1215.5844 \\ 1027.5823 & 1027.5047 \\ 913.4914 & 913.4505 \\ 2390.3230 & 2390.2190 \\ 1494.8687 & 1494.7790 \\ 1443.7831 & 1443.6994 \\ 2125.1988 & 2125.0579 \\ 1586.8867 & 1586.7900 \\ 1114.6175 & 1114.5618\end{array}$

ppm

83

76

45

44

60

58

66

61

50

\section{Miss Sequence}

1 M. STRSVSSSSYR . R

1 R. SVSSSSYRR.M

O R. SYVTTSTR. T

1 R. SYVTTSTRTYSLGSA LRPSTSR.S

O R. TYSLGSALRPSTSR. S

- R. SLYSSSPGGAYVTR. S

O R. LLQDSVDF SLADAINTEFK. N

1 R. TNEKVELQELNDR . F

O K. VELQELNDR . F 


\section{M10}

$4700 \mathrm{MS} / \mathrm{MS}$ Precursor 1557.99 Spec \#1 MC[BP = 86.1, 6500]

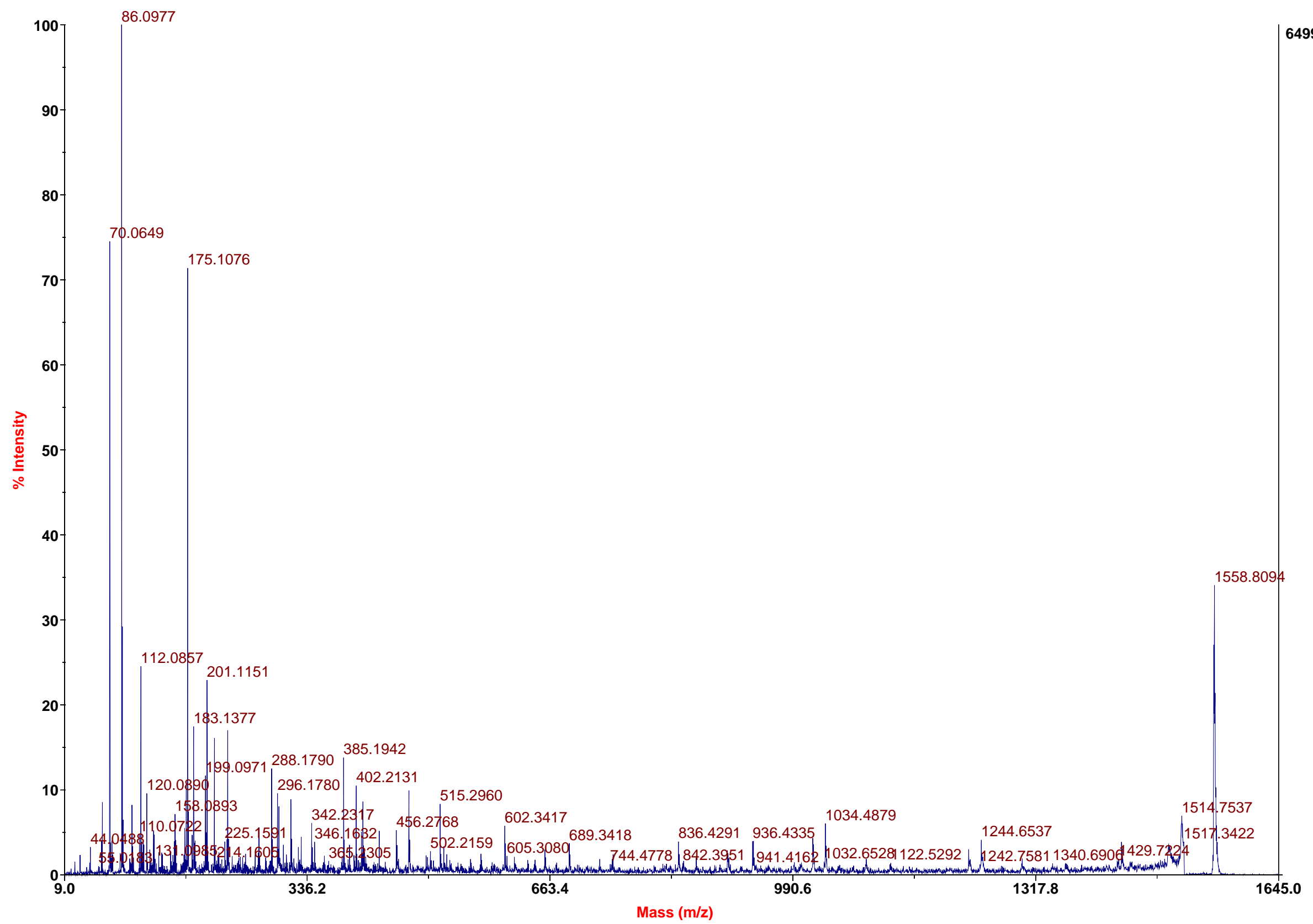




\section{Protein View}

Match to: VIME_MOUSE score: 337

Vimentin. - Mus musculus (Mouse).

Found 1n search of ppw_J2_129990855309.txt

Nominal mass $\left(M_{x}\right): 53524$; Calculated pI value: 5.06

NCBI BLAST search of VIME_MOUSE aga1nst nr

unformatted sequence string for pasting into other applications

Taxonony: Mus musculus

Var1able modif1cat1ons: Carban1domethyl (C), Dean1dated (NQ), Ox1dat1on (M)

cleavage by Trypsin: cuts c-term side of KR unless next residue $1 \mathrm{~s}$

sequence coverage: 368

Matched peptides shown 1n Bold Red

1 STRSVSSSSY RRMFGGSGTS SRPSSNRSYV TTSTRTYSLG SALRPSTSRS

51 LYSSSPGGAY VTRSSAVRLR SSVPGVRLLQ DSVDESLADA INTEFKNTRT

101 NEKVELQELN DRFANYIDKV RFLEQQNKIL LAELEQLKGO GKSRLGDLYE

151 EEMRELRRQV DQLTNDKARV EVERDNLAED IMRLREKLQE EMLQREEAES

201 TLQSFRQDVD NASLARLDLE RKVESLQEEI AFLKKLHDEE IOELOAQIQE

251 QHVQIDVDVS KPDLTAALRD VRQQYESVAA KNLQEAEEWY KSKFADLSEA

301 ANRNNDALRQ AKQESNEYRR QVQSLTCEVD ALKGTNESLE ROMREMEENF

351 ALEAANYQDT IGRLQDEIQN MKEEMARHLR EYQDLLNVKM ALDIEIATYR

401 KLLEGEESRI SLPLPTFSSL NLRETNLESL FLVDTHSKRT LLIKTVETRD

451 GOVINETSQH HDDLE

\section{Show predicted peptides also}

\section{Sort Peptides By}

Start - End
$36-49$
$50-63$
$100-112$
$104-112$
$145-154$
$170-183$
$188-195$
$222-234$
$282-291$
$294-303$
$313-320$
$345-363$
$364-377$
$390-400$
$410-423$

9) Residue Number

\section{Obse rved}

1495.8760

1444.7904

1587.8940

1115. 6248

1254.6362

1688.9337

1046.5817

1533. 9412

1309.6765

1093.5818

1081.5563

2201,1218

1734.9233

1295.7373

1557.9924

\section{Mr (expt)}

1494.8687

1443.7831

1586.8867

1114.6175

1253.6289

1687.9264

1045,5744

1532.9339

1308.6692

1092.5745

1080.5490

2200,1145

1733.9160

1294.7300

1556.9851
Increasing Mass

Mr (calc)
1494.7790
1443.6994
1586.7900
1114.5618
1253.5598
1687.8199
1045.5226
1532.8450
1308.5986
1092.5200
1080.4948
2199.9742
1733.8076
1294.6591
1556.8926

Decreasing Mass 
Sample M11

4700 Reflector Spec \#1 MC=>NR(2.00)[BP = 1516.8, 43842]






\section{Protein View}

Match to: Q3TVP6_Mouse score: 160 Expect: $9.2 e-12$

osteoclast-like cell cDNA, RIKEN full-length enriched library, clone:I420044I15 product:actil Found $1 \mathrm{n}$ search of pmf_k2_129990849010.txt

Nom1nal mass $\left(M_{x}\right): 41724$; Calculated pI value: 5.30

NCBI BLAST search of Q3TVP6 MOUSE aga1nst nr

unformatted sequence str1ng for pasting 1nto other applications

Taxonony: Mus musculus

Links to retrieve other entries contalning this sequence from NCBI Entrez:

(no taxonomy information for this entry)

Var1able mod1f1cat1ons: Carban1domethyl (C), Dean1dated (NQ), ox1dat1on (M)

cleavage by Trypsin: cuts c-term side of KR unless next residue $15 \mathrm{~F}$

Number of mass values searched: 125

Number of mass values matched: 37

sequence coverage: $82 \%$

Matched peptides shown in Bold Red

1 MDDDIAALVV DNGSGMCKAG FAGDDAPRAV FPSIVGRPRH QGVMVGMGQK

51 DSYVGDEAQS KRGILTLKYP IEHGIVTNWE DMEKIWHHTF YNELRVAPEE

101 HPVLLTEAPL NPKANREKMT OIMEETENTP AMYVAIQAVL SLYASGRTTG

151 IVMDSGDGVT HTVPIYEGYA LPHAILRLDL AGRDLTDYLM KILTERGYSF

201 TTTAEREIVR DIKEKLCYVA LDFEOEMATA ASSSSLEKSY ELPDGOVITI

251 GNERFRCPEA LFQPSELGME SCGIHETTFN SIMKCDVDIR KDLYANTVLS

301 GGTTMYPGIA DRMQKEITAL APSTMKIKII APPERKYSVW IGGSILASLS

351 TFQQMWISKQ EYDESGPSIV HRKCF

Show predicted peptides also

\section{Sort Peptides By}

$\begin{aligned} & \text { Start } \text { End } \\ & 19-28 \\ & 29-39 \\ & 40-50 \\ & 40-50 \\ & 40-50 \\ & 40-50 \\ & 51-62 \\ & 69-84 \\ & 69-84 \\ & 85-95 \\ & 85-95 \\ & 96-113 \\ & 96-116\end{aligned}$

- Residue Number

\section{Obse rved}

976.5027

1198.7769

1171.6378

1173.6477

1187.6346

1203.6329

1354. 7119

1961.0471

1977.0839

1515.8496

1516.8330

1954.1995

2295.3442

$\begin{array}{rr}\text { Mr (expt) } & \text { Mr (calc) } \\ 975.4955 & 975.4410 \\ 1197.7696 & 1197.6982 \\ 1170.6305 & 1170.5638 \\ 1172.6404 & 1172.5318 \\ 1186.6273 & 1186.5587 \\ 1202.6256 & 1202.5536 \\ 1353.7046 & 1353.6161 \\ 1960.0398 & 1959.9036 \\ 1976.0766 & 1975.8986 \\ 1514.8423 & 1514.7419 \\ 1515.8257 & 1515.7259 \\ 1953.1922 & 1953.0571 \\ 2294.3369 & 2294.2382\end{array}$

Increasing Mass

Decreasing Mass

56
60
57
93
58
60
65
69
90
66
66
69
43

\section{Miss Sequence}

- K. AGF AGDDAPR. A

O R. AVFPSIVGRPR . H

- R. HQGVMVGMGQK. D

- R. HQGVMVGMGQK. D

- R. HQGVMV GMGQK . D

R. HQGVMV GMGQK . D

K. DSYVGDEAQSKR . G oxidation

2 ox1dat 1

K. YPIEHGIVTNWEDMEK . I

K. YPIEHGIVTNWEDMEK . I

K. IWHHTFYNELR. V K. IWHATFYNELR.V Dearn R. VAPEEHPVLLTEAPLNPKANR , E 


\section{M11}

4700 MS/MS Precursor 1515.85 Spec \#1 MC[BP = 110.1, 4191]






\section{M11}

4700 MS/MS Precursor 1132.59 Spec \#1 MC[BP = 175.1, 3537]






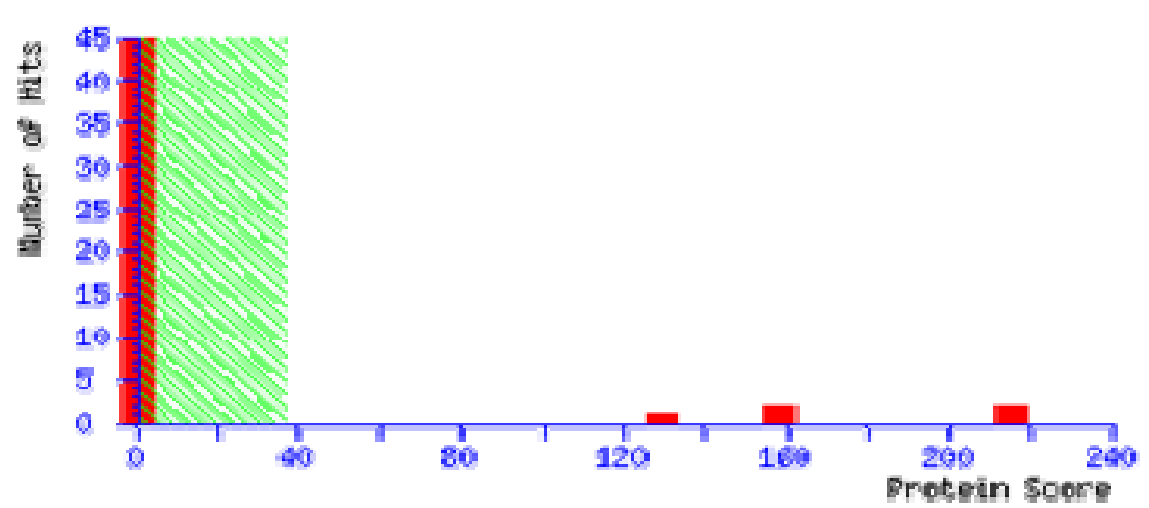

\section{Peptide Summary Report}

\section{Format As Peptide Summary}

Significance threshold $\mathrm{p}<0.05$

Standard scoring $\bigcirc$ MudPIT scoring

Show pop-ups Suppress pop-ups
Max. number of hits AUTO

Ions score or expect cut-off 0

Sort unassigned Decreasing Score $\underline{\text { Help }}$

Show sub-sets 0

Require bold red

\section{Select All}

\section{Select None}

Search Selected

\section{Error tolerant}

1. $\frac{\text { CAA27396 }}{\text { MMACTBR2 NID: - Mus musculus }}$

Matches: $13(8)$

Sequences: $11(8)$

emPAI : 2.23

Check to include this hit in error tolerant search

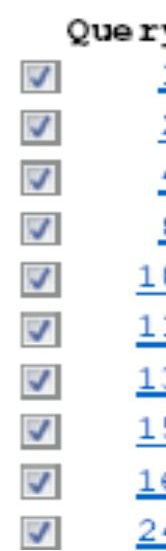

$\begin{array}{rrr}\text { Observed } & \text { Mr(expt) } & \text { Mr(calc) } \\ 923.6166 & 922.6093 & 922.5600 \\ 945.6028 & 944.5956 & 944.5444 \\ 998.5252 & 997.5179 & 997.4790 \\ 1132.5944 & 1131.5871 & 1131.5197 \\ 1171.6378 & 1170.6305 & 1170.5638 \\ 1187.6346 & 1186.6273 & 1186.5587 \\ 1354.7119 & 1353.7046 & 1353.6161 \\ 1515.8496 & 1514.8423 & 1514.7419 \\ 1791.0154 & 1790.0081 & 1789.8846 \\ 1954.1995 & 1953.1922 & 1953.0571\end{array}$

$\begin{array}{lcc}\text { ppm } & \text { Miss } & \text { Score } \\ 53.5 & 1 & 18 \\ 54.2 & 0 & 37 \\ 39.0 & 0 & 8 \\ 59.6 & 0 & 58 \\ 57.0 & 0 & 81 \\ 57.8 & 0 & (18) \\ 65.4 & 1 & 39 \\ 66.3 & 0 & 27 \\ 69.0 & 0 & 60 \\ 69.2 & 0 & 42\end{array}$

E

0.641

$0.028 \quad 1$

$37 \quad 1$

$0.00054 \quad 1$

2. $1 e-06 \quad 1$

5. 41

$0.037 \quad 1$

0.451

$0.00021 \quad 1$

$0.0051 \quad 1$

Peptide

K. IIAPPERK. Y

R. AVFPSIVGR . S

R. DLTDYLMK. I

R. GYSETTTAER . E

R. HQGVMV GMGQK. D

R. HQGVMVGMGQK. D + Oxida

K. DSYVGDEAQSKR . G

K. IWHHTFYNELR. V

K. SYELPDGQVITIGNER . F

R. VAPEEHPVLLTEAPLNPK . A 


\section{Protein View}

Match to: CAA27396 score: 215

\section{MMACTBR2 NID: - Mus musculus}

Found 1n search of ppr_K2_129990855410.txt

Nom1nal mass $\left(\mathrm{M}_{\mathrm{I}}\right)$ : 39161; Calculated pI value: 5.78

NCBI BLAST search of CAA27396 aga1nst $\mathrm{nr}$

unformatted sequence string for pasting 1nto other applications

Taxonory: Mus musculus

Links to retrieve other entries containing this sequence from NCBI Entrez:

(no taxonomy information for this entry)

Var1able modif1cations: Carbanidonethyl (C), Dean1dated (NQ), ox1dation (M)

cleavage by Trypsin: cuts c-term side of $K R$ unless next residue $1 s \mathrm{P}$

sequence coverage:

448

Matched peptides shown 1n Bold Red

1 PRAVFPSIVG RSRHQGVMVG MGQKDSYVGD EAQSKRGILT LKYPIEHGIV

51 TNWDDMEKIW HHTFYNELRV APEEHPVLLT EAPLNPKANR EKMTOIMFET

101 FNTPAMYVAI QAVLSLYASG RTTGIVMDSG DGVTHTVPIY EGYALPHAIL

151 RLDLAGRDLT DYLMKILTER GYSFTTTAER EIVRDIKEKL CYVALDFEQE

201 MATAASSSSL EKSYELPDGQ VITIGNERFR CPEALFQPSF LGMESCGIHE

251 TTFNSIMKCD VDIRKDLYAN TVLSGGTTMY PGIADRMOKE ITALAPSTMK

301 IKIIAPPERK YSVWIGGSIL ASLSTFOQMN ISKQEYDESG PSIVHRKCF

Show predicted peptides also

Sort Peptides By

(9) Residue Number

Increasing Mass

Decreasing Mass

$\begin{aligned} \text { Start } & - \text { End } \\ 3 & -11 \\ 14 & -24 \\ 14 & -24 \\ 25 & -36 \\ 59 & -69 \\ 70 & -87 \\ 122 & -151 \\ 158 & -165 \\ 171 & -180 \\ 213 & -228\end{aligned}$

Obse rved

945. 6028

1171.6378

1187.6346

1354.7119

1515.8496

1954.1995

3183.8391

998. 5252

1132.5944

1791,0154

\begin{tabular}{rr} 
Mr (expt) & \multicolumn{1}{c}{ Mr (calc) } \\
944.5956 & 944.5444 \\
1170.6305 & 1170.5638 \\
1186.6273 & 1186.5587 \\
1353.7046 & 1353.6161 \\
1514.8423 & 1514.7419 \\
1953.1922 & 1953.0571 \\
3182.8318 & 3182.6071 \\
997.5179 & 997.4790 \\
1131.5871 & 1131.5197 \\
1790.0081 & 1789.8846
\end{tabular}

ppm

0 R.AVFPSIVGR.S (Ions score 37)

570 R. HQGVMVGMGQK. D (Ions score 81)

58

65

66

69

71

39

60

69
0 R. HQGVMVGMGQK. D oxidation (M)

1 K.DSYVGDEAQSKR.G (IOnS score 39)

0 K. IWHHTFYNELR.V (I OnS score 27)

0 R. VAPEEHPVLLTEAPLNPK.A (IONS score 42)

0 R. TTGIVMDSGDGVTHTVP IYEGYALPHA ILR. L (IONS

0 R. DLTDYLMK. I (Ions score 8)

0 R. GYSFTTTAER. E (IONS score 58)

o K. SYELPDGQVITIGNER. F (IONS score 60) 


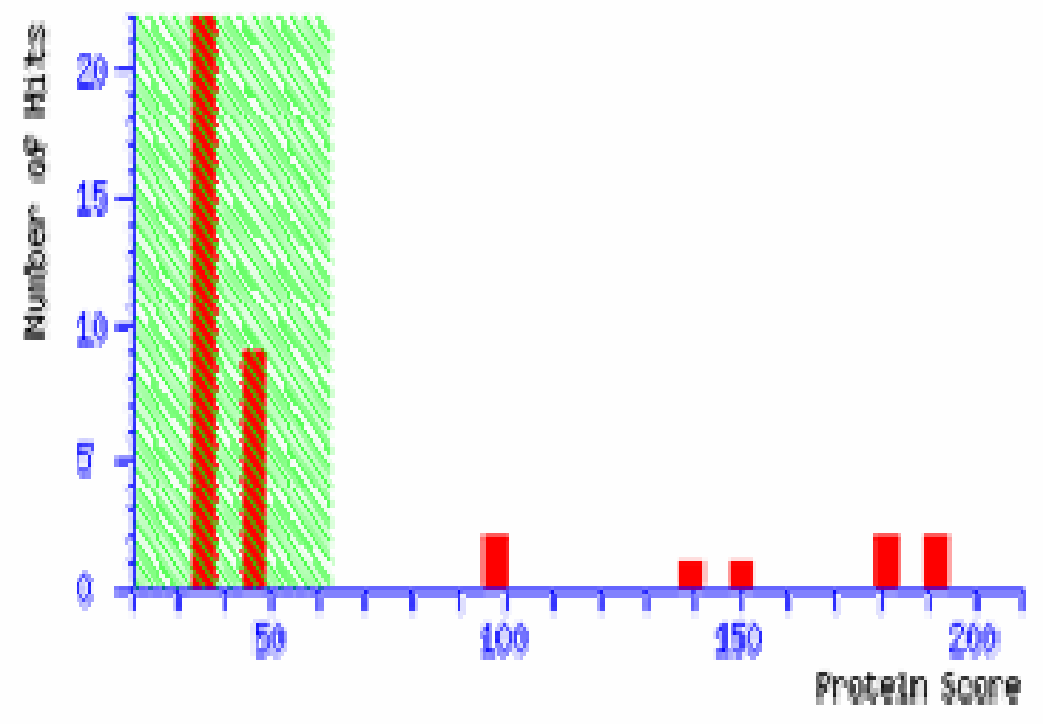

Concise Protein Summary Report

Format As Concise Protein Summary .

Significance threshold $\mathrm{p} \times 0.05 \quad$ Max. number of hits AUTO

\section{Re-Search All Search Unmatched}

1. Mixture 1 Total score: 192 Expect: 5.8e-15 Matches: 76 Components (only one famlly menber shown for each component): P8C7C7 Mouse Mass: 64961 Score: 154 Expect: 3.7e-11 Matches: 54

2 days neonate thymus thynic cells coNa, RIKEN full-length enriched library, clone:c920028B14 product:albumin 1, AME49490 Mass: 50514 Score: 98 Expect: 1.4e-05 Matches: 27

Mmo89889 NID: - Mus musculus 


\section{Protein View}

Match to: Q8C7C7_Mouse score: 154 Expect: $3.7 e-11$

2 days neonate thymus thymic cells cDNA, RIKEN full-length enriched library, clone:C920028B14 pro Found $1 \mathrm{n}$ search of pmf_L2_129990849011.txt

Nomlnal mass $\left(M_{x}\right): 64961$; calculated pI value: 5.49

NCBI BLAST search of Q8C7C7 MOUSE aga1nst nr

unformatted sequence string for past1ng 1nto other applications

Taxonony: Mus musculus

Links to retrieve other entries conta1n1ng this sequence from NCBI Entrez:

(no taxonomy information for this entry)

Var1able mod1f1cat1ons: Carbam1domethyl (C), Dean1dated (NQ), ox1dat1on (M) cleavage by Trypsin: cuts C-term side of KR unless next residue $15 \mathrm{P}$

Number of mass values searched: 164

Number of mass values matched: $\mathbf{5 4}$

sequence coverage: 718

Matched peptides shown 1n Bold Red

1 NRYNDLGEQH FKGLVLIAFS QYLQKCSYDE HAKLVQEVTD FAKTCVADES

51 AANCDKSLHT LFGDKLCAIP NLRENYGELA DCCTKQEPER NECFLQHKDD

101 NPSLPPFERP EAEAMCTSEK ENPTTFMGHY LHEVARRHPY FYAPELLYYA

151 EQYNEILTQC CAEADKESCL TPKLDGVKEK ALVSSVRQRM KCSSMQKFGE

201 RAFKAWAVAR LSOTFPNADF AEITKLATDL TKVNKECCHG DLLECADDRA

251 ELAKYMCENQ ATISSKLQTC CDKPLLKKAH CLSEVEHDTM PADLPAIAAD

301 FVEDQEVCKN YAEAKDVELG TFLYEYSRRH PDYSVSLLLR LAKKYEATLE

351 KCCAEANPPA CYGTVLAEFO PLVEEPKNLV KTNCDLYEKL GEYGFONAIL

401 VRYTQKAPQV STPTLVEAAR NLGRVGTKCC TLPEDQRLPC VEDYLSAILN

451 RVCLLHEKTP VSEHVTKCCS GSLVERRPCE SALTVDETYV PKEFKAETET

501 FHSDICTLPE KEKQIKKQTA LAELVKHKFK ATAEQLKTVM DDEAQFLDTC

551 CKAADKDTCF STEGPNLVTR CKDALA

Show predicted peptides also

\section{Sort Peptides By}

Start - End
$1-12$
$3-12$
$3-25$
$13-25$
$13-33$
$13-33$
$26-33$
$34-43$
$57-65$
$57-73$

- Residue Number

Observed
1520.8607
1250.6466
2711.5913
1479.8870
2470.3696
2472.3184
1009.4545
1149.6699
1017.5802
1898.0306

\section{Mr (expt)} 1519.8534 1249.6393 2710.5840 1478.8797 2469.3623 2471. 3111 1008,4472 1148.6626 1016.5729 1897.0233
Increasing Mass

Mr (calc)
1519.7168
1249.5727
2710.4119
1478.8497
2469.2362
2471.2042
1008.3971
1148.6077
1016.5291
1897.0244

Decreasing Mass

ppm
90
53
64
20
51
43
50
48
43
-1

\section{Miss Sequence}

$\begin{array}{ll}1 & -. \text { NRYNDLGEQHFK. G } \\ 0 & \text { R. YNDLGEQHFK. G } \\ 1 & \text { R. YNDLGEQHFKGLVLIAFSQYLQK. C } \\ 0 & \text { K. GLVLIAFSQYLQK. C } \\ 1 & \text { K. GLVLIAFSQYLQKCSYDEHAK. L Carb } \\ 1 & \text { K. GLVLIAFSQYLQKCSYDEHAK. L Carb } \\ 0 & \text { K. CSYDEHAK. L CaYbam1doInethYl } \\ 0 & \text { K. LVQEVTDFAK. T } \\ 0 & \text { K. SLHTLFGDK. L } \\ 1 & \text { K. SLHTLFGDKLCAIPNLR. E }\end{array}$




\section{Protein View}

Match to: AAB49490 score: 98 Expect: $1.4 e-05$

MMU89889 NID: - Mus musculus

Found in search of pmf_L2_129990849011.txt

Nom1nal mass $\left(M_{x}\right)$ : 50514; Calculated pI value: 7.32

NCBI BLAST search of A.AB 49490 aga1nst nr

unformatted sequence str1ng for past1ng 1nto other applications

Taxonony: Mus musculus

Var1able mod1f1cat1ons: Carban1domethyl (C), Dean1dated (NQ), ox1dat1on (M)

cleavage by Trypsin: cuts C-term side of $\mathrm{KR}$ unless next residue $1 \mathrm{~s} F$

Number of mass values searched: 164

Number of mass values matched: 27

sequence coverage: 568

Matched peptides shown in Bold Red

1 ALNILVLLGL CWSLAVASPL PTANGRVAEV ENGTKPDSDV PEHCLDTWSF

51 DAATMDHNGT MLFFKGEFVW RGHSGTRELI SAFWKNPITS VDAAFRGPDS

101 VFLIKEDKVW VYPPEKKENG YPKLFOEEFP GIPYPPDAAV ECHRGECQSE

151 GVLFFQGNRK WFWDFATRTQ KERSWSTVGN CTAALRWLER YYCFQGNKFL

201 RFNPVTGEVP PRYPLDARDY FVSCPGQGHG RPRNGTAHGN STHPMHSRCS

251 PDPGLTALLS DHRGATYAFT GSHYWRLDSS RDGWHSWPIA HHWPQGPSTV

301 DAAFSWDDKV YLIQGTQVYV FLTKGGNNLV SGYPKRLEKE LGSPPGISLE

351 TIDAAFSCPG SSRLYVSSGR RLWWLDLKSG AOATWTEVSW PHEKVDGALC

401 LDKSLGPNTC SSNGSSLYEI HGPNLYCYSS IDKLNAAKSL PQPQKVSILG

$451 \operatorname{CSQ}$

Show predicted peptides also

\section{Sort Peptides By}

$\begin{aligned} \text { Start } & - \text { End } \\ 84 & -96 \\ 86 & -96 \\ 97 & -105 \\ 97 & -108 \\ 109 & -116 \\ 109 & -117 \\ 124 & -144 \\ 145 & -159 \\ 145 & -160 \\ 160 & -168 \\ 161 & -168 \\ 191 & -198\end{aligned}$

$\begin{array}{rrr}\text { Observed } & \text { Mr(expt) } & \text { Mr (calc) } \\ 1504.8777 & 1503.8704 & 1503.7834 \\ 1190.6776 & 1189.6703 & 1189.6091 \\ 975.5921 & 974.5848 & 974.5437 \\ 1347.7872 & 1346.7799 & 1346.7082 \\ 1017.5802 & 1016.5729 & 1016.5331 \\ 1145.6559 & 1144.6486 & 1144.6281 \\ 2472.3184 & 2471.3111 & 2471.1580 \\ 1727.8855 & 1726.8782 & 1726.7733 \\ 1855.9944 & 1854.9871 & 1854.8683 \\ 1256.6888 & 1255.6815 & 1255.6138 \\ 1128.5824 & 1127.5751 & 1127.5189 \\ 1079.5121 & 1078.5048 & 1078.4542\end{array}$

Decreasing Mass

ppm
58
51
42
53
39
18
62
61
64
54
50
47

\section{Miss Sequence}
1 R. WKNPITSVDAAFR, G
K. NPITSVDAAFR. G
R. GPDSVFLIK. E
R. GPDSVF LIKEDK.V
K. VWVYPPEK, K
K. VWVYPPEKK . E
K. LFOEEFPGIPYPPDAAVECHR , G Carban 1
R. GECQSEGVLFFQGNR. $\mathrm{K}$ Carban1dometh
R. GECQSEGVLFFQGNRK. W Carbamidomet
R. KWFWDFATR . T
K. WFWDFATR. T
R. YYCEQGNK. F Carbamidomethyl
(C) 


\section{M12}

4700 MS/MS Precursor 1479.89 Spec \#1 MC[BP = 175.1, 4373]






\section{M12}

4700 MS/MS Precursor 1609.88 Spec \#1 MC[BP = 120.1, 3103]

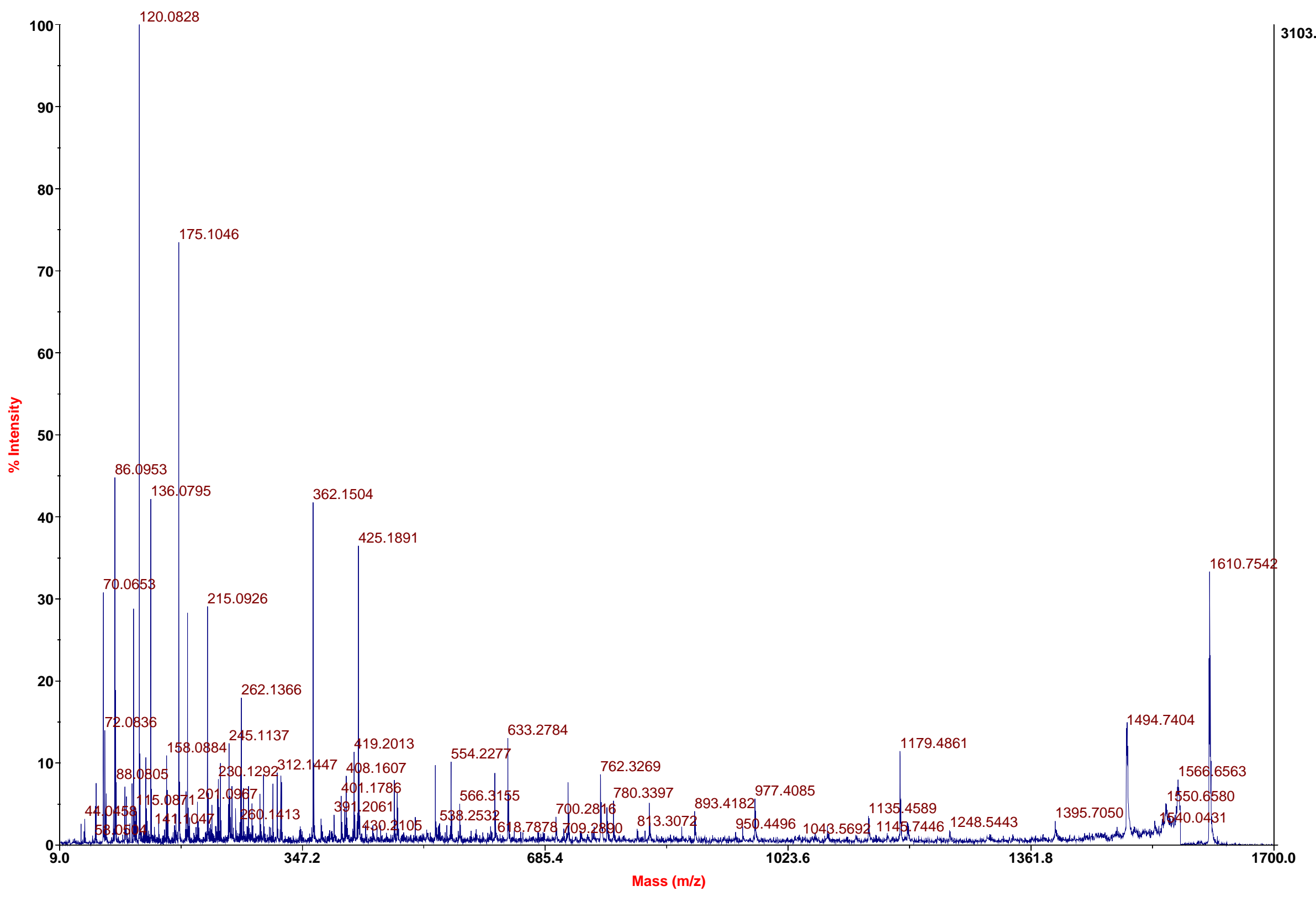




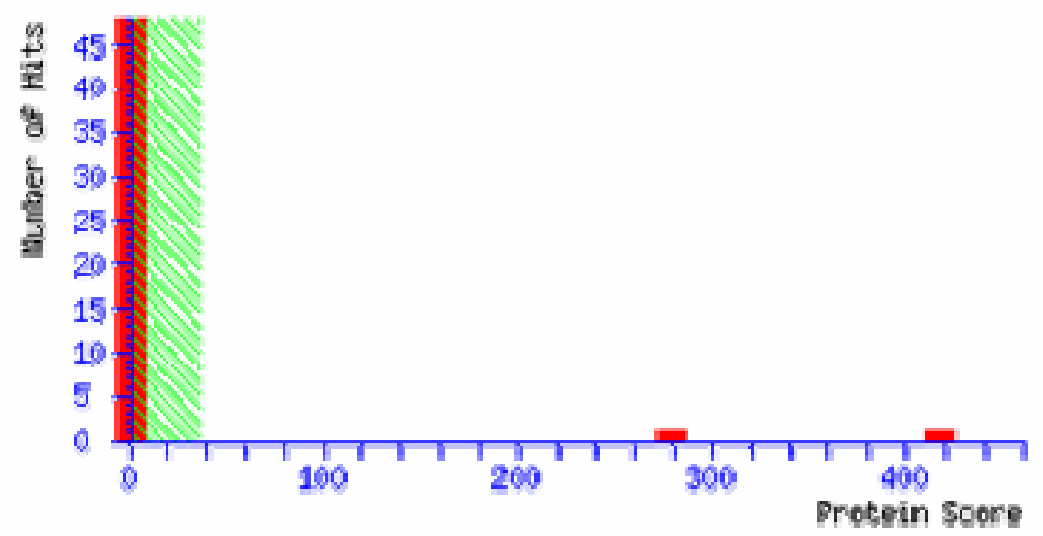

\section{Peptide Summary Report}

\section{Format As Peptide Summary}

Significance threshold $p<0.05$

Standard scoring MudPIT scoring - Ions score or expect cut-off 0

Show pop-ups Suppress pop-ups Sort unassigned Decreasing Score
Help

Show sub-sets 0

Require bold red

\section{$\square$ Error tolerant}

1. 3UKP2 MOUSE Mass: 51285 Score: 417 Matches: $10(9)$ Sequences: 10(9) emPAI: 1.72

14 days embryo l1ver cDNA, RIKEN full-length enrlched l1brary, clone:I530016F13 product:henopex1n, full 1nsert si

$\square$ check to include this hit in error tolerant search

\begin{tabular}{|c|c|c|c|c|c|c|c|c|c|c|}
\hline Query & Observed & $\mathrm{Mr}$ (expt) & $\mathrm{Mr}(\mathrm{calc})$ & ppm & Miss & Score & Expect & Rank & Unique & Peptide \\
\hline 目 & 1100.5388 & 1099.5315 & 1099.4757 & 50.8 & 0 & 45 & 0.01 & 1 & $\mathrm{U}$ & R.DYFVSCPGR.G + Carbamidomethyl (C) \\
\hline$\underline{3}$ & 1128.5824 & 1127.5751 & 1127.5189 & 49.9 & 0 & 18 & $4 \cdot 3$ & 1 & $\mathrm{U}$ & K. WFWDFATR. T \\
\hline$\underline{5}$ & 1212.7012 & 1211.6939 & 1211.6299 & 52.9 & 0 & 65 & 5. $1 e-05$ & 1 & $\mathrm{U}$ & R. FNPVTGEVPPR. Y \\
\hline$\underline{9}$ & 1504.8777 & 1503.8704 & 1503.7834 & 57.9 & 1 & 56 & 0.00054 & 1 & $\mathrm{U}$ & R. WKNPITSVDAAFR. G \\
\hline$\underline{10}$ & 1516.7863 & 1515.7790 & 1515.6895 & 59.1 & 0 & 53 & 0.0016 & 1 & $\mathrm{U}$ & R. GATYAFTGSHYWR . L \\
\hline$\underline{12}$ & 1638.8927 & 1637.8854 & 1637.7832 & 62.4 & 0 & 79 & 3. $4 e-06$ & 1 & $\mathrm{U}$ & R. CSPDPGLTALLSDHR.G + Carbamidome \\
\hline$\underline{14}$ & 1727.8855 & 1726.8782 & 1726.7733 & 60.7 & 0 & 112 & $2 e-09$ & 1 & $\mathrm{U}$ & R. GECQSEGVLFFQGNR. K + Carbamidon \\
\hline
\end{tabular}




\section{Protein View}

Match to: Q3UKP2 MousE score: 417

14 days embryo liver cDNA, RIKEN full-length enriched library, clone:I530016F13 product: hemopexin, full inst Found 1n search of ppw_L2_129990855611.txt

Nom1nal mass $\left(M_{x}\right)$ : 51285; Calculated pI value: 7.92

NCBI BLAST search of Q3UKP2_MOUSE aga1nst nr

Unformatted sequence string for pasting into other applications

Taxonony: Mus musculus

Links to retrieve other entries contalning this sequence from NCBI Entrez:

(no taxonomy information for this entry)

Var1able mod1f1cations: Carbanidomethyl (C), Dean1dated (NQ), ox1dation (M) cleavage by Trypsin: cuts c-term side of KR unless next residue 1 s $P$

sequence coverage: $28 \%$

Matched peptides shown 1n Bold Red

1 MARTAVALNI LVLLGLCWSL AVASPLPTAN GRVAEVENGT KPDSDVPEHC

51 LDTWSFDAAT MDHNGTMLFF KGEFVWRGHS GTRELISARW KNPITSVDAA

101 FRGPDSVFLI KEDKVWVYPP EKKENGYPKL FQEEFPGIPY PPDAAVECHR

151 GECQSEGVLF FQGNRKWFWD FATRTQKERS WSTVGNCTAA LRWLERYYCF

201 QGNKFLRFNP VTGEVPPRYP LDARDYFVSC PGRGHGRPRN GTAHGNSTHP

251 MHSRCSPDPG LTALLSDHRG ATYAFTGSHY WRLDSSRDGW HSWPIAHHWP

301 QGPSTVDAAF SWDDKVYLIQ GTQVYVELTK GGNNLVSGYP KRLEKELGSP

351 PGISLETIDA AFSCPGSSRL YVSSGRRLW LDLKSGAQAT WTEVSWPHEK

401 VDGALCLDKS LGPNTCSSNG SSLYFIHGPN LYCYSSIDKL NAAKSLPQPQ

451 KVNSILGCSQ

\section{Show predicted peptides also}

\section{Sort Peptides By}

\section{(9) Residue Number}

\section{Observed}

1504.8777

2472. 3184

1727.8855

1855. 9944

1128.5824

1212.7012

1100.5388

1638.8927

1516.7863

2448. 3303

\section{Mr (expt) \\ Mr (calc)}

1503.8704

2471. 3111

1726.8782

1854. 9871

1127.5751

1211.6939

1099.5315

1637.8854

1515. 7790

2447.3230
1503.7834

2471. 1580

1726.7733

1854.8683

1127.5189

1211. 6299

1099.4757

1637.7832

1515. 6895

2447.1638
Decreasing Mass

ppm
58
62
61
64
50
53
51
62
59
65

\section{Miss Sequence}

1 R. WKNPITSVDAAFR.G (Ions score 56)

- K. LFQEEFPGIPYPPDAAVECHR.G Carbanidomethy

- R. GECQSEGVLFFQGNR.K Carban1domethyl (C)

1 R. GECQSEGVLFFQGNRK.W Carbam1domethyl (C)

- K. WFWDFATR. T (Ions score 18)

- R. FNPVTGEVPPR. Y (IOns score 65)

- R. DYFVSCPGR.G Carban1domethyl (C) (Ions

- R. CSPDPGLTALLSDHR.G Carban1domethy 1 (C)

- R. GATYAFTGSHYWR. L (IONS score 53)

- K. ELGSPPGISLETIDAAF SPGSSR. I Carban1 dome 
Sample M13

4700 Reflector Spec \#1 MC=>NR(2.00)[BP = 1100.5, 28668]

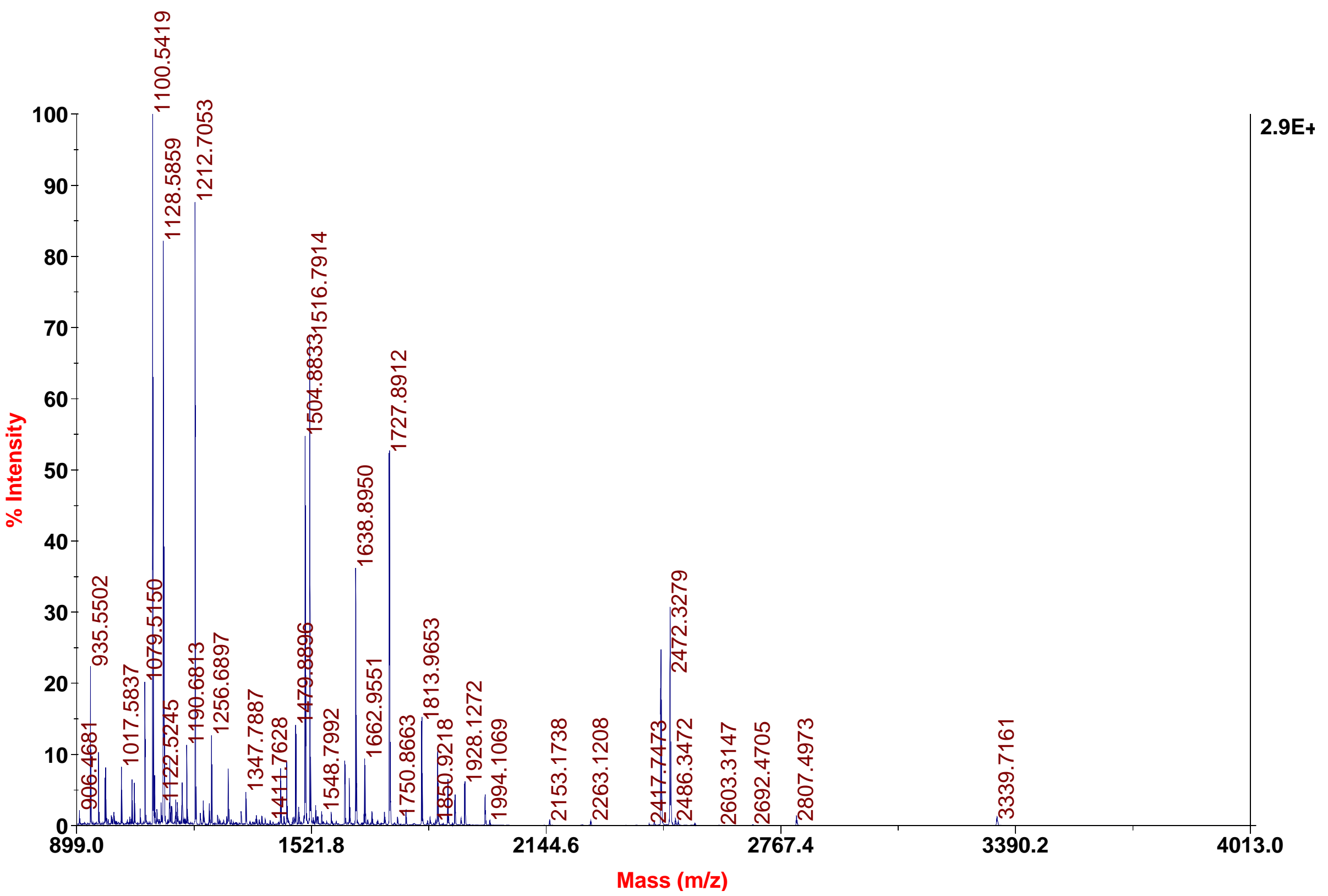




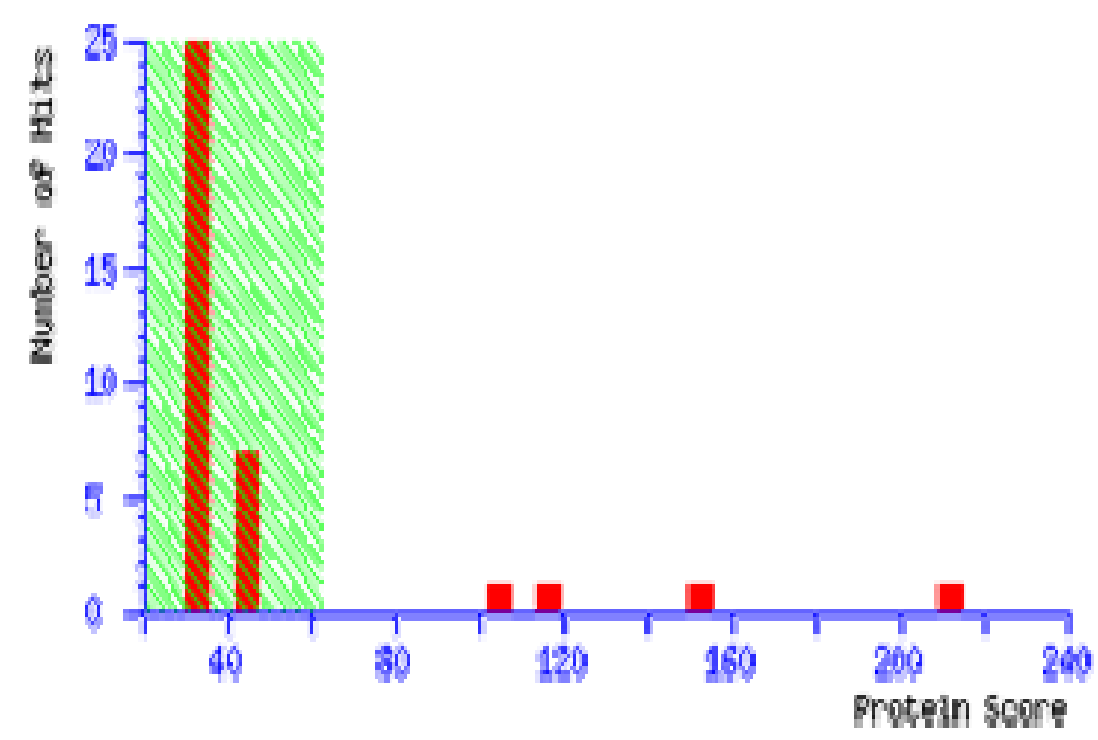

\section{Concise Protein Summary Report}

Format As Concise Protein Summary - $\underline{\underline{\text { Help }}}$

Significance threshold $p<\overline{0.05}$ Max. number of hits AUTO

Re-Search All

Search Unmatched

1. Mixture 1 Total score: 212 Expect: $5.8 e-17$ Matches: 70

Components (only one famlly member shown for each component):

Q3UKR2 MOUSE Mass: 51285 Score: 152 Expect: 5.8e-11 Matches: 31

14 days enbryo l1ver cDNA, RIKEN full-length enriched l1brary, clone:I530016F13 product:hemopexin, full 1nse Q8C7C7_Mouse Mass: 64961 Score: 114 Expect: 3.7e-07 Matches: 46

2 days neonate thymus thyn10 cells cona, RIKEN full-length enriched library, clone:C920028B14 product:album1 


\section{rotein View}

atch to: Q3UKP2_MOUSE Score: 152 Expect: 5.8e-11

4 days embryo liver cDNA, RIKEN full-length enriched library, clone:I530016F13 product:hemopexin, ound in search of pmf_M2_129990849012.txt

om1nal mass $\left(M_{x}\right): 51285$; calculated pI value: 7.92

CBI BLAST search of Q3UKP2 MOUSE aga1nst nr

nformatted sequence string for pasting 1nto other applications

axonorny: Mus musculus

inks to retrieve other entries conta1ning this sequence from NCBI Entrez:

(no taxonomy information for this entry)

ar1able mod1f1cat1ons: Carban1domethyl (C), Dean1dated (NQ), ox1dat1on

leavage by Trypsin: cuts c-term side of KR unless next residue $1 \mathrm{~s} F$

umber of mass values searched: 126

umber of mass values matched: 31

equence coverage: 638

atched peptides shown in Bold Red

1 MARTAVALNI LVLLGLCWSL AVASPLPTAN GRVAEVENGT KPDSDVPEHC

51 LDTWSFDAAT MDHNGTMLFF KGEFVWRGHS GTRELISARW KNPITSVDAA

101 FRGPDSVFLI KEDKVWVYPP EKKENGYPKL FOEEEPGIPY PPDAAVECHR

151 GECQSEGVLF FQGNRKWFWD FATRTQKERS WSTVGNCTAA LRWLERYYCF

201 QGNKFLRFNP VTGEVPPRYP LDARDYFVSC PGRGHGRPRN GTAHGNSTHP

251 MHSRCSPDPG LTALLSDHRG ATYAFTGSHY WRLDSSRDGW HSWPIAHHWP

301 QGPSTVDAAF SWDDKVYLIQ GTQVYVELTK GGNNLVSGYP KRLEKELGSP

351 PGISLETIDA AFSCPGSSRL YVSSGRRLWW LDLKSGAQAT WTEVSWPHEK

401 VDGALCLDKS LGPNTCSSNG SSLYFIHGPN LYCYSSIDKL NAAKSLPQPQ

451 KVNSILGCSQ

Show predicted peptides also

\section{Sort Peptides By}

Start - End

$90-102$

$92-102$

$103-111$

$103-114$

$112-122$

$115-122$

$115-123$
- Residue Number

Increasing Mass

Decreasing Mass

ppm
61
53
44
54
55
43
52

$\begin{array}{rrr}\text { Observed } & \text { Mr(expt) } & \text { Mr(calc) } \\ 1504.8826 & 1503.8753 & 1503.7834 \\ 1190.6791 & 1189.6718 & 1189.6091 \\ 975.5937 & 974.5864 & 974.5437 \\ 1347.7883 & 1346.7810 & 1346.7082 \\ 1389.7819 & 1388.7746 & 1388.6976 \\ 1017.5838 & 1016.5765 & 1016.5331 \\ 1145.6945 & 1144.6872 & 1144.6281\end{array}$

Miss Sequence

1 R. WKNPITSVDAAFR, G

- K. NPITSVDAAFR. G

O R. GPDSVFLIK. E

1 R. GPDSVF LIKEDK.V

1 K. EDKVWVYPPEK. K

o K. VWVYPPEK. K

1 K. VWVYPPEKK. E 


\section{Protein View}

Match to: Q8C7C7_MousE score: 114 Expect: $3.7 e-07$

2 days neonate thymus thymic cells cDNA, RIKEN full-length enriched library, clone:C92002 Found in search of pmf_M2_129990849012.txt

Nomlnal mass $\left(M_{x}\right)$ : 64961; calculated pI value: 5.49

NCBI BLAST search of Q8C7C7 MOUSE aga1nst nr

unformatted sequence str1ng for pasting 1nto other applications

Taxonorny: Mus musculus

Links to retrieve other entries contalning this sequence from NCBI Entrez:

(no taxonomy information for this entry)

Var1able mod1f1cat1ons: Carban1domethyl (C), Dean1dated (NQ), ox1dation cleavage by Trypsin: cuts c-term side of KR unless next residue $1 s$ P Number of mass values searched: 126

Number of mass values matched: 46

sequence coverage: 698

Matched peptides shown 1n Bold Red

1 NRYNDLGEQH FKGLVLIAES QYLQKCSYDE HAKLVQEVTD FAKTCVADES

51 AANCDKSLHT LFGDKLCAIP NLRENYGELA DCCTKQEPER NECFLQHKDD

101 NPSLPPFERP EAEAMCTSEK ENPTTEMGHY LHEVARRHPY FYAPELLYYA

151 EOYNEILTQC CAEADKESCL TPKLDGVKEK ALVSSVRORM KCSSMOKFGE

201 RAFKAWAVAR LSQTFPNADF AEITKLATDL TKVNKECCHG DLLECADDRA

251 ELAKYMCENO ATISSKLQTC CDKPLLKKAH CLSEVEHDTM PADLPAIAAD

301 FVEDQEVCKN YAEAKDVFLG TFLYEYSRRH PDYSVSLLLR LAKKYEATLE

351 KCCAEANPPA CYGTVLAEFQ PLVEEPKNLV KTNCDLYEKL GEYGFQNAIL

401 VRYTQKAPQV STPTLVEAAR NLGRVGTKCC TLPEDQRLPC VEDYLSAILN

451 RVCLLHEKTP VSEHVTKCCS GSLVERRPCF SALTVDETYV PKEFKAETET

501 FHSDICTLPE KEKQIKKQTA LAELVKHKPK ATAEQLKTVM DDFAQFLDTC

551 CKAADKDTCF STEGPNLVTR CKDALA

Show predicted peptides also

Sort Peptides By

Start - End
$1-12$
$13-25$
$13-33$
$13-33$
$13-33$
$34-43$
$34-56$
$57-65$

(6) Residue Number

Observed
1520.8362
1479.8887
2415.3196
2470.3232
2472.3269
1149.6681
2457.3489
1017.5838

\section{Obse rved}

1520.8362

2415.3196

2470.3232

2472,3269

2457.3489

1017.5838

\section{Mr (expt)}

1519.8289

1478.8814

2414.3123

2469.3159

2471.3196

1148.6608

2456.3416

1016.5765
Increasing Mass

Mr (calc)
1519.7168
1478.8497
2414.1828
2469.2362
2471.2042
1148.6077
2456.1199
1016.5291

Decreasing Mass

$\begin{array}{rcl}\text { ppm } & \text { Miss } & \text { Sequence } \\ 74 & 1 & \text {-. NRYNDLGEQHFK.G } \\ 21 & 0 & \text { K. GLVLIAFSQYLQK. C } \\ 54 & 1 & \text { K. GLVLIAFSQYLQKCSYDEHAK } \\ 32 & 1 & \text { K. GLVLIAFSQYLQKCSYDEHAK } \\ 47 & 1 & \text { K. GLVLIAFSQYLQKCSYDEHAK } \\ 46 & 0 & \text { K. LVQEVTDFAK. T } \\ 90 & 1 & \text { K. LVQEVTDFAKTCVADESA.ANCI } \\ 47 & 0 & \text { K. SLHTLFGDK. L }\end{array}$




\section{M13}

4700 MS/MS Precursor 1638.89 Spec \#1 MC[BP = 312.2, 11579]

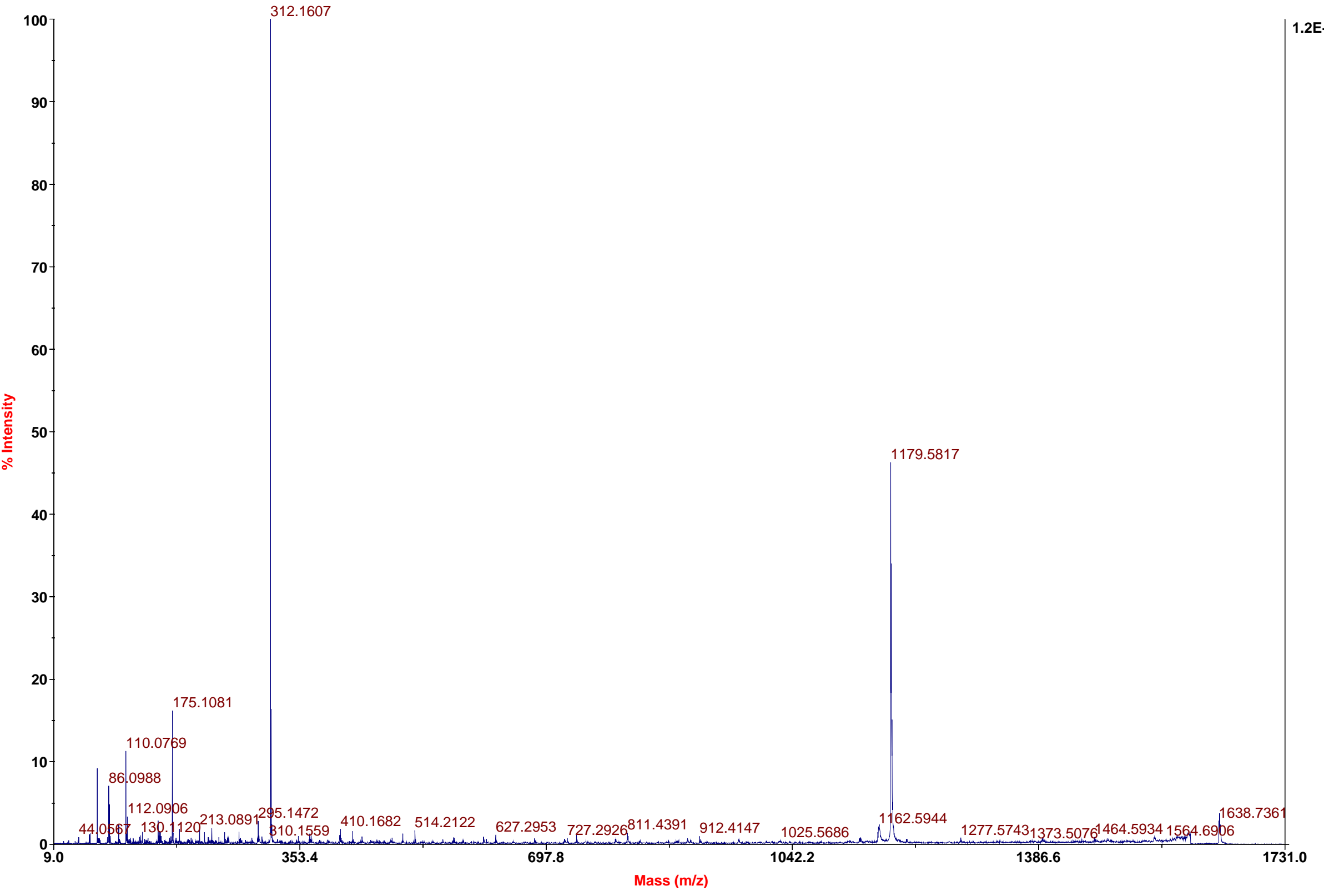






\section{Peptide Summary Report}

Format As Peptide Summary

Significance threshold $p<0.05 \quad$ Max. number of hits AUTO

Standard scoring MudPIT scoring $\odot$ Ions score or expect cut-off 0

Show pop-ups 9 Suppress pop-ups Sort unassigned Decreasing Score
Help

Show sub-sets 0

Require bold red

\section{Select All Select None Search Selected $\square$ Error tolerant}

1. Q3UKP2 MOUSE Mass: 51285 Score: 404 Matches: 15(9) Sequences: 15(9) emPAI: 2.80

14 days embryo 11ver cDNA, RIKEN full-length enr1ched l1brary, clone:I530016F13 product:hemopex1n, full 1nsert s

$\square$ check to include this hit in error tolerant search

\begin{tabular}{|c|c|c|c|c|c|c|c|c|c|c|}
\hline Query & Observed & $\operatorname{Mr}($ expt) & $\mathrm{Mr}(\mathrm{calc})$ & ppm & Miss & Score & Expect & Rank & Unique & Peptide \\
\hline$\underline{2}$ & 1079.5145 & 1078.5072 & 1078.4542 & 49.2 & 0 & 49 & 0.0041 & 1 & $\mathrm{U}$ & R. YYCEQGNK. F + Carbamidomethyl (C) \\
\hline$\underline{3}$ & 1100.5393 & 1099.5320 & 1099.4757 & 51.2 & 0 & 36 & 0.067 & 1 & $\mathrm{U}$ & R. DYFVSCPGR.G \\
\hline 4 & 1128.5852 & 1127.5779 & 1127.5189 & 52.4 & 0 & 21 & 2.5 & 1 & $\mathrm{U}$ & K. WFWDFATR. T \\
\hline
\end{tabular}




\section{Protein View}

Match to: Q3UKP2_MOUSE score: 404

14 days embryo liver cDNA, RIKEN full-length enriched library, clone:I530016F13 product:hemopexin, full insert sequen Found in search of ppw_M2_129990855812.txt

Nom1nal mass $\left(M_{r}\right)$ : 51285; Calculated pI value: 7.92

NCBI BLAST search of Q3UKP2 MOUSE against $\mathrm{nr}$

unformatted sequence string for pasting into other applications

Taxonony: Mus musculus

Links to retrieve other entries containing this sequence from NCBI Entrez:

(no taxonomy information for this entry)

Var1able modif1cat1ons: Carbanidonethyl (C), Dean1dated (NQ), oxidation (M)

cleavage by Trypsin: cuts C-term side of $K R$ unless next residue is $P$

sequence coverage: $35 \%$

Matched peptides shown in Bold Red

1 MARTAVALN LVLLGLCWSL AVASPLPTAN GRVAEVENGT KPDSDVPEHC

51 LDTWSFDAAT MDHNGTMLFF KGEFVWRGHS GTRELISARW KNPITSVDAA

101 FRGPDSVFLI KEDKVWVYPP EKKENGYPKL FQEEFPGIPY PPDAAVECHR

151 GECQSEGVLF FQGNRKWFWD FATRTQKERS WSTVGNCTAA LRWLERYYCF

201 QGNKFLRFNP VTGEVPPRYP LDARDYFVSC PGRGHGRPRN GTAHGNSTHP

251 MHSRCSPDPG LTALLSDHRG ATYAFTGSHY WRLDSSRDGW HSWPIAHHWP

301 QGPSTVDAAF SWDDKVYLIQ GTQVYVELTK GGNNLVSGYP KRLEKELGSP

351 PGISLETIDA AFSCPGSSRL YVSSGRRLW LDLKSGAQAT WTEVSWPHEK

401 VDGALCLDKS LGPNTCSSNG SSLYFIHGPN LYCYSSIDKL NAAKSLPQPQ

451 KVNSILGCSQ

Show predicted peptides also

Sort Peptides By

(9) Residue Number $\bigcirc$ Increasing Mass

Decreasing Mass

$\begin{array}{rrrr}\text { Start - End } & \text { Observed } & \text { Mr(expt) } & \text { Mr(calc) } \\ 90-102 & 1504.8826 & 1503.8753 & 1503.7834 \\ 92-102 & 1190.6791 & 1189.6718 & 1189.6091 \\ 115-123 & 1145.6945 & 1144.6872 & 1144.6281 \\ 130-150 & 2472.3269 & 2471.3196 & 2471.1580 \\ 151-165 & 1727.8903 & 1726.8830 & 1726.7733 \\ 151-166 & 1855.9941 & 1854.9868 & 1854.8683 \\ 166-174 & 1256.6886 & 1255.6813 & 1255.6138 \\ 167-174 & 1128.5852 & 1127.5779 & 1127.5189 \\ 197-204 & 1079.5145 & 1078.5072 & 1078.4542 \\ 208-218 & 1212.7028 & 1211.6955 & 1211.6299\end{array}$

ppm
61
53
52
65
64
64
54
52
49
54

Miss Sequence

1 R. WKNPITSVDAAFR.G (Ions score 69)

0 K. NPITSVDAAFR. G (IONS score 44)

1 K. WWVYPPEKK.E (Ions score 6)

0 K. LFQEEFPGIPYPPDAAVECHR.G Carban1domethy 1 (C) (IOI

0 R. GECQSEGVLFFQGNR . K Carbanidomethy 1 (C) (IOnS scor

1 R. GECQSEGVLFFQGNRK.W Carbamidomethyl (C) (IOnS SCO

1 R. KWFWDFATR.T (Ions score 19)

0 K. WFWDFATR. T (Ions score 21)

0 R. YYCFQGNK.F Carbam1domethyl (C) (Ions score 49)

$208-218$

$1212.7028 \quad 1211.6955 \quad 1211.6299$

0 R. FNPVTGEVPPR. Y (Ions score 39) 


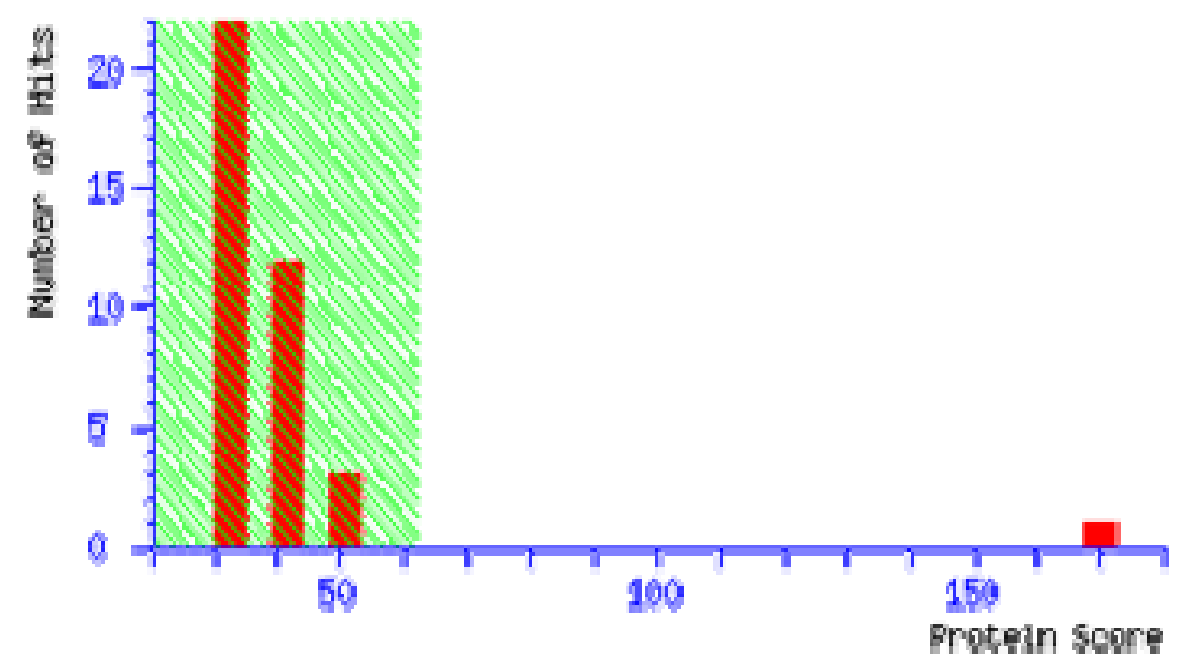

\section{Concise Protein Summary Report}
Format As
Concise Protein Summary
$\underline{\text { Help }}$
Significance threshold $p<\overline{0.05}$ Max. number of hits AUTO

Re-Search All

Search Unmatched

1. Q3UKP2 MOUSE Mass: 51285 Score: 170 Expect: $9.2 e-13$ Matches: 25

14 days embryo l1ver cDNA, RIKEN full-length enrlched l1brary, clone:I530016F13 product:hemopex1] A.AH11246 Mass: 51308 Score: 170 Expect: 9.2e-13 Matches: 25

BC011246 NID: - Mus musculus

A.AB 49490 Mass: 50514 Score: 157 Expect: $1.8 \mathrm{e}-11$ Matches: 24

MMU89889 NID: - Mus musculus

Q8K1U6_MOUSE Mass: 31027 Score: 110 Expect: 9.2e-07 Matches: 15

Hemopexin (Fragnent). - Mus musculus (Mouse). 


\section{Protein View}

Match to: Q3UKP2_MOUSE score: 170 Expect: $9.2 e-13$

14 days embryo liver cDNA, RIKEN full-length enriched library, clone:I530016F13 product: hemopexin, Found $1 \mathrm{n}$ search of pmf_N2_129990849013.txt

Nom1nal mass $\left(M_{x}\right): 51285$; Calculated pI value: 7.92

NCBI BLAST search of Q3UKP2 MOUSE aga1nst nr

unformatted sequence string for pasting 1nto other applications

Taxonony: Mus musculus

L1nks to retrieve other entries conta1ning this sequence from NCBI Entrez:

(no taxonomy information for this entry)

Var1able mod1f1cat1ons: Carban1domethyl (C), Dean1dated (NQ), ox1dat1on

cleavage by Trypsin: cuts c-term side of $\mathrm{KR}$ unless next residue $1 \mathrm{~s} F$

(M)

Number of mass values searched: 108

Number of mass values matched: 25

sequence coverage: $51 \%$

Matched peptides shown in Bold Red

1 MARTAVALNI LVLLGLCWSL AVASPLPTAN GRVAEVENGT KPDSDVPEHC

51 LDTWSFDAAT MDHNGTMLFF KGEFVWRGHS GTRELISAFW KNPITSVDAA

101 FRGPDSVFLI KEDKVWVYPP EKKENGYPKL FOEEFPGIPY PPDAAVECHR

151 GECQSEGVLF FQGNRKWFWD FATRTQKERS WSTVGNCTAA LRWLERYYCF

201 QGNKFLRFNP VTGEVPPRYP LDARDYFVSC PGRGHGRPRN GTAHGNSTHP

251 MHSRCSPDPG LTALLSDHRG ATYAFTGSHY WRLDSSRDGW HSWPIAHHWP

301 QGPSTVDAAF SWDDKVYLIQ GTQVYVELTK GGNNLVSGYP KRLEKELGSP

351 PGISLETIDA AFSCPGSSRL YVSSGRRLWW LDLKSGAQAT WTEVSWPHEK

401 VDGALCLDKS LGPNTCSSNG SSLYFIHGPN LYCYSSIDKL NAAKSLPQFQ

451 KVNSILGCSQ

Show predicted peptides also

\section{Sort Peptides By}

Start - End
$90-102$
$92-102$
$103-111$
$103-114$
$115-122$
$115-123$
$130-150$
$151-165$
$151-165$
$151-166$
$166-174$

$\begin{array}{rrr}\text { Observed } & \text { Mr(expt) } & \text { Mr (calc) } \\ 1504.8705 & 1503.8632 & 1503.7834 \\ 1190.6692 & 1189.6619 & 1189.6091 \\ 975.5863 & 974.5790 & 974.5437 \\ 1347.7714 & 1346.7641 & 1346.7082 \\ 1017.5754 & 1016.5681 & 1016.5331 \\ 1145.6865 & 1144.6792 & 1144.6281 \\ 2472.3081 & 2471.3008 & 2471.1580 \\ 1670.8501 & 1669.8428 & 1669.7519 \\ 1727.8776 & 1726.8703 & 1726.7733 \\ 1855.9836 & 1854.9763 & 1854.8683 \\ 1256.6799 & 1255.6726 & 1255.6138\end{array}$

Decreasing Mass

ppm
53
44
36
42
34
45
58
54
56
58
47

\section{Miss Sequence}

1 R. WKNPITSVDAAFR. G

K. NPITSVDAAFR. G

R. GPDSVFLIK, E

R. GPDSVF LIKEDK.V

K. VWVYPPEK. K

K. VWVYPPEKK.E

K. LFQEEFPGIPYPPDAAVECHR.G Carban1

R. GECQSEGVLFFQGNR . $K$

R. GECQSEGVLFFQGNR . K Carban1dometh

R. GECQSEGVLFFQGNRK . W carbamidomet R. KWFWDF ATR . T 


\section{M14}

4700 MS/MS Precursor 1638.88 Spec \#1 MC[BP = 312.2, 3114]

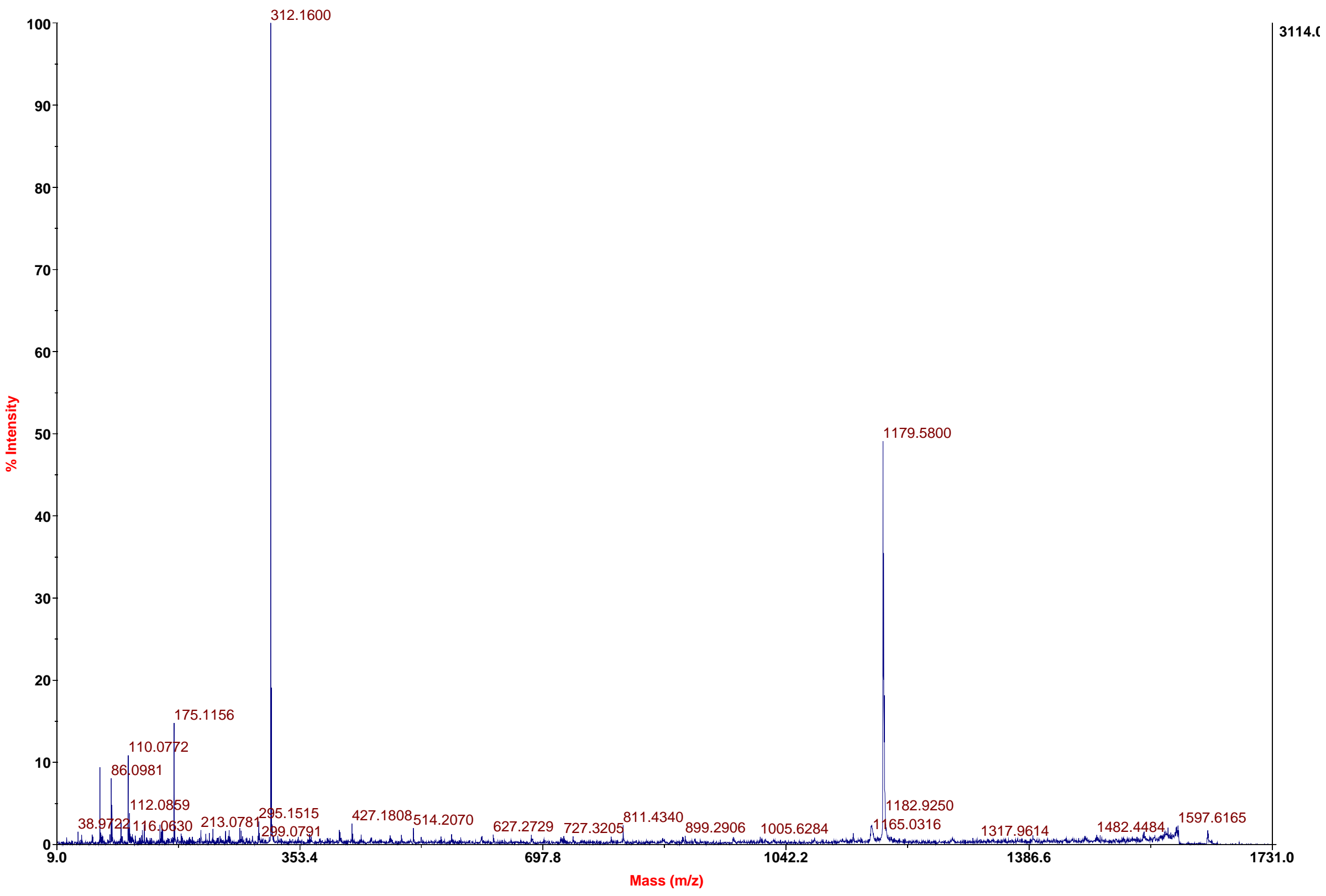




\section{M14}

4700 MS/MS Precursor 1051.75 Spec \#1 MC[BP = 861.0, 1795]

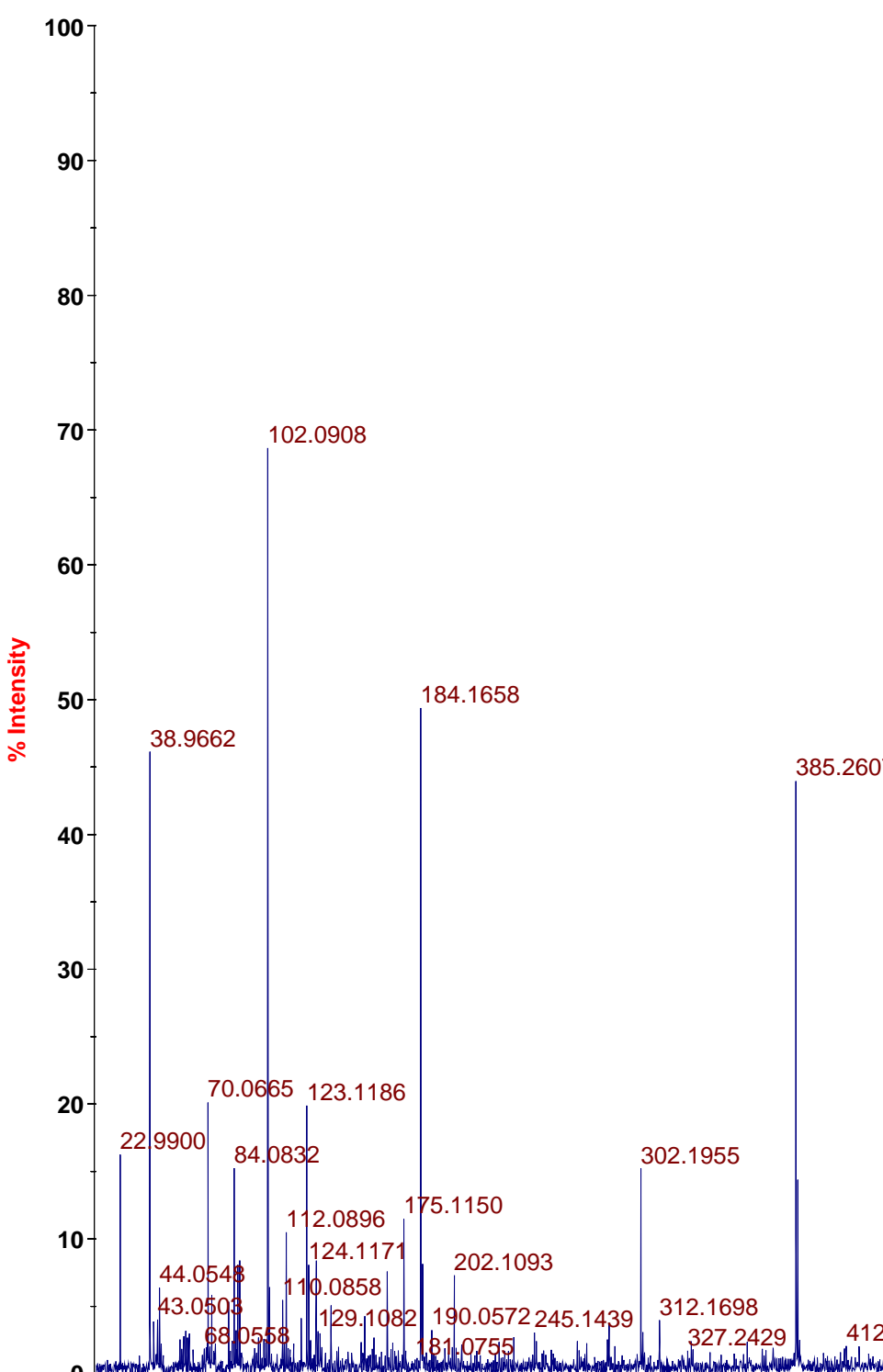

861.0516

467.3367

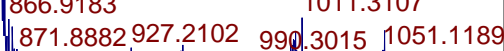

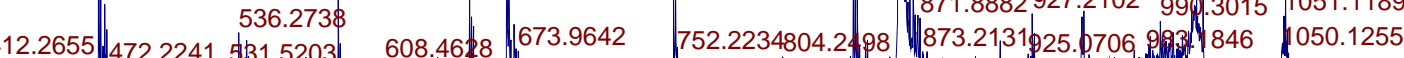
752.2234804 .2498 873.2131925.0706 983.4846 1050.1255 


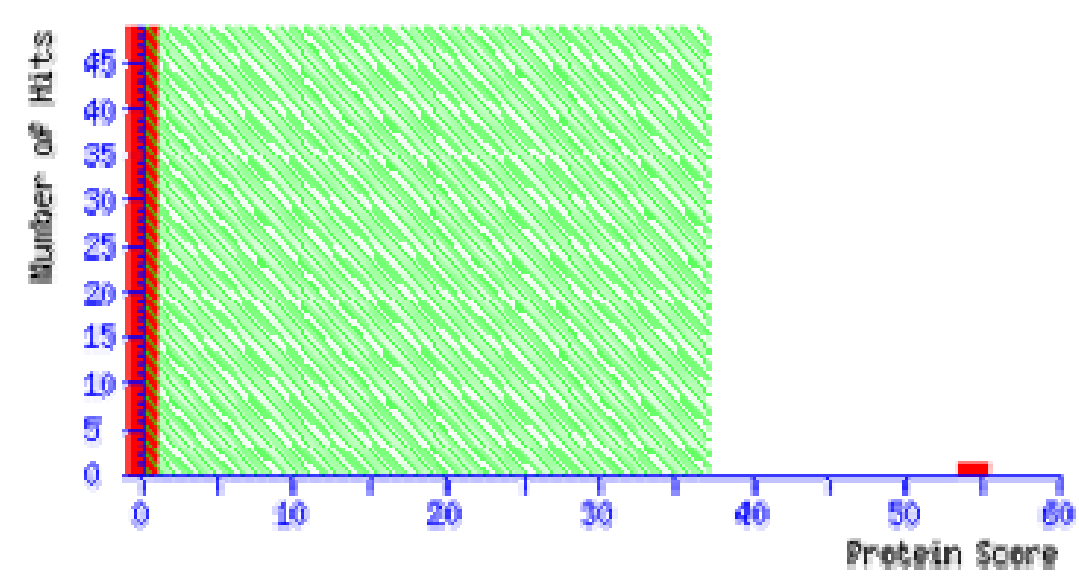

\section{Peptide Summary Report}

\section{Format As Peptide Summary}

Significance threshold $p<0.05$

Standard scoring

Show pop-ups Suppress pop-ups
MudPIT scoring s 0

\author{
Max. number of hits AUTO \\ Ions score or expect cut-off 0 \\ Sort unassigned Decreasing Score
}

\section{Help}

Show sub-sets 0

Require bold red

\section{Error tolerant}
Q3UKP2_MOUSE
Mass: 51285 Score: 55
Matches: $15(1)$
Sequences: 15 (1)
emPAI : 0.56

14 days embryo l1ver cDNA, RIKEN full-length enr1ched library, clone:I530016F13 product:henopex1n, full 1nser

$\square$ check to include this hit in error tolerant search

\begin{tabular}{|c|c|c|c|}
\hline Query & Observed & $\operatorname{Mr}(e x p t)$ & $\mathrm{Mr}(\mathrm{calc})$ \\
\hline$\underline{2}$ & 975.5863 & 974.5790 & 974.5437 \\
\hline$\underline{3}$ & 1017.5754 & 1016.5681 & 1016.5331 \\
\hline$\underline{6}$ & 1079.5037 & 1078.4964 & 1078.4542 \\
\hline$\underline{7}$ & 1100.5300 & 1099.5227 & 1099.4757 \\
\hline$\underline{8}$ & 1128.5742 & 1127.5669 & 1127.5189 \\
\hline
\end{tabular}

$\begin{array}{lcc}\text { ppm } & \text { Miss } & \text { Score } \\ 36.3 & 0 & 34 \\ 34.4 & 0 & 18 \\ 39.1 & 0 & 25 \\ 42.8 & 0 & 27 \\ 42.6 & 0 & 4\end{array}$

Expect Rank Unique

$\begin{array}{rrr}0.095 & 1 & \mathrm{U} \\ 4.7 & 1 & \mathrm{U} \\ 1 & 1 & \mathrm{U} \\ 0.59 & 1 & \mathrm{U} \\ 2 e+02 & 1 & \mathrm{U}\end{array}$

\section{Peptide}

R. GPDSVFLIK . E

K. VWVYPPEK. K

R. YYCFQGNK. F + Carbamidomethyl

R. DYFVSCPGR.G + Carbamidomethy 1

K. WFWDFATR, T 


\section{Protein View}

Match to: Q3UKP2_MOOSE score: 55

14 days embryo liver cDNA, RIKEN full-length enriched library, elone:I530016F13 product:hemopexin, full : Found in search of PPE_N2_129990855913.txt

Nominal mass $\left(\mathrm{M}_{\mathrm{r}}\right)$ : 51285 ; Calculated pI value: 7.92

NCBI BLAST search of Q3UKF2 MOUSE against $n r$

unformatted sequence string for pasting into other applications

Taxonony: Mus musculus

Links to retrleve other entries contalning this sequence from MCBI Entrez:

(no taxonomy 1nformation for this entry)

Varlable modifications: Carbanidonethy (C), Deanidated (No), oxidation (M)

cleavage by Trypsin: cuts c-term. side of KR unless next residue is $\mathrm{F}$

Sequence Coverage: 348

Matched peptides shown in Bold Red

1 MARTAVALN LVLLGLCNSL AVASPLETAN GRVAEVENGT KFDSDVPEHC

51 LDTWSFDAAT MDHNGTMLFF KGEEWRGHS GTRELISAEW KNPITSVDAA

101 FRGPDSVFLI KEDKVWVYPP EKKENGYPKL FOEEFPGIPY PPDAAVECHR

151 GECOSEGVLF FOGNRKWEWD FATRTQKERS WSTVGNCTAA LRWLERYYCF

201 QGNKELEFNP VTGEVPPRYF LDARDYFVSC PGRGHGRERN GTAHGNSTHF

251 MHSRCSPDPG LTALLSDHRG ATYAFTGSHY WRLDSSRDCW HSWPIAHHWF

301 QGPSTVDAAF SWDDKVYLIQ GTQVYVELTK GGNLVSGYP KRLEKELGSP

351 PGISLETIDA AFSCPGSSRL YVSSGRRLW LDLKSGAOAT WTEVSWPHEK

401 VDGALCLDKS LGPNTCSSNG SSLYFIHGPN LYCYSSIDKL NAAKSLPOPQ

451 KVNSI LGCSQ 
Sample M15

4700 Reflector Spec \#1 MC=>NR(2.00)[BP = 1100.5, 15842]

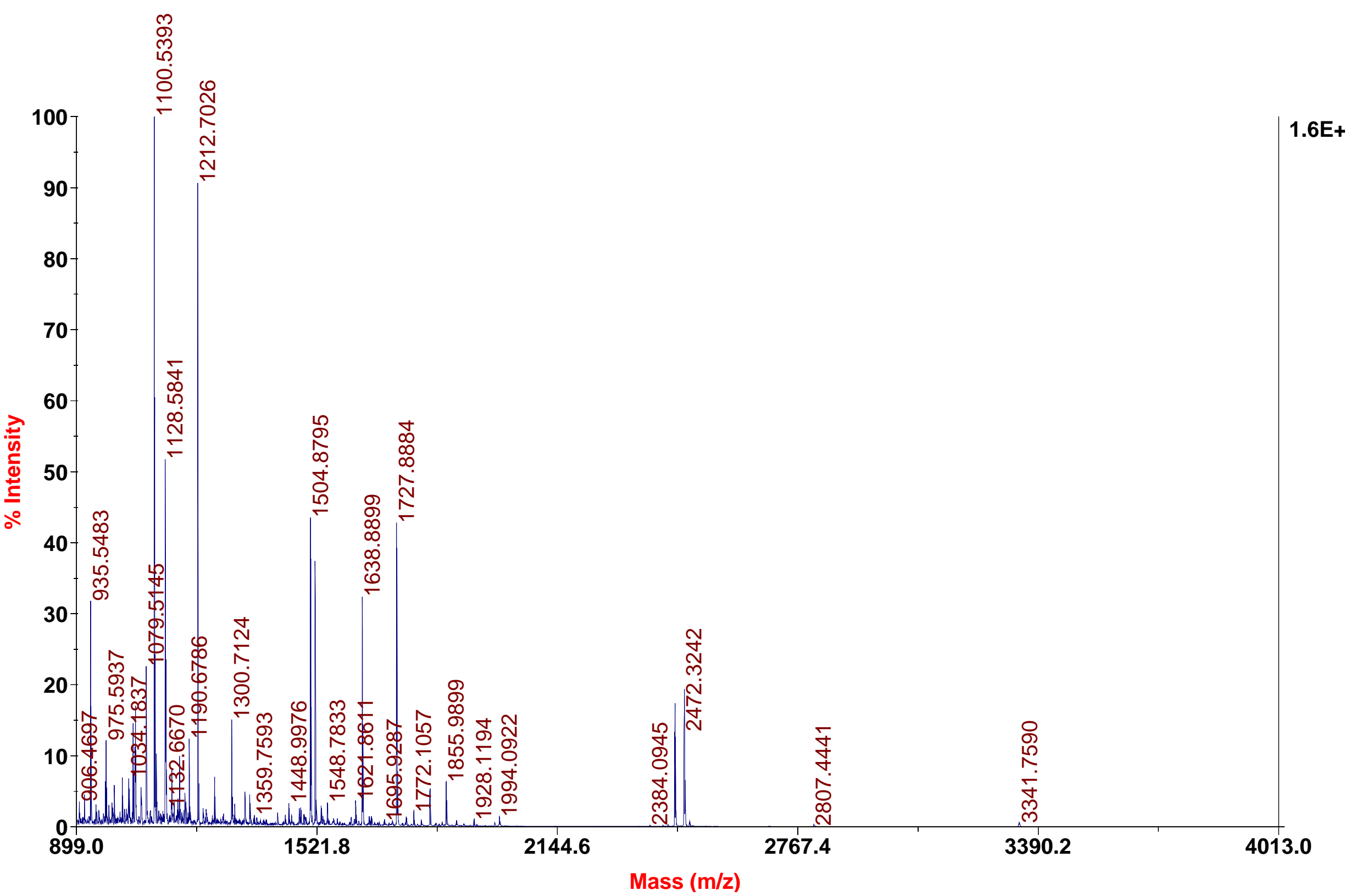




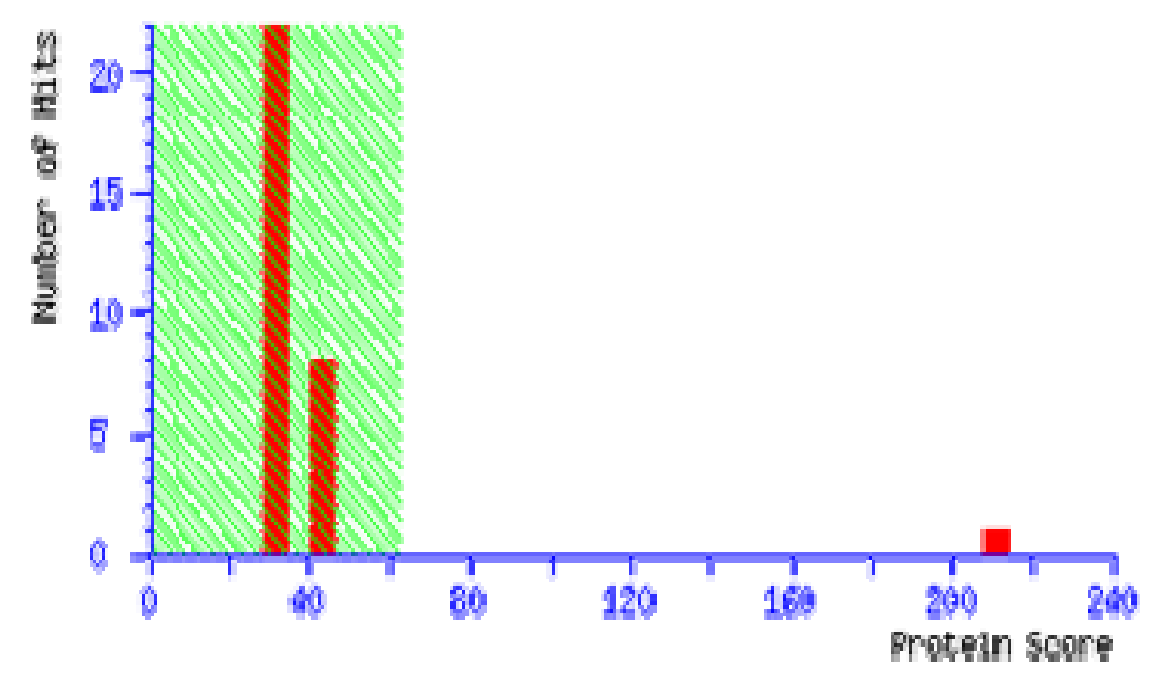

\section{Concise Protein Summary Report}
Format As
Concise Protein Summary
$\underline{\text { Help }}$
Significance threshold $p<0.05$
Max. number of hits AUTO

Re-Search All

Search Unmatched

1. Q3UKP2 MOUSE Mass: 51285 Score: 211 Expect: $7.3 e-17$ Matches: 29

14 days embryo l1ver CDNA, RIKEN full-length enr1ched 11brary, clone:I530016F13 product:hemopexin, fu ARH11246 Mass: 51308 Score: 211 Expect: $7.3 e-17$ Matches: 29

BC011246 NID: - Mus musculus

AAB 49490 Mass: 50514 Score: 196 Expect: 2.3e-15 Matches: 27

MMU89889 NID: - Mus musculus

Q8K1U6_MOUSE Mass: 31027 Score: 134 Expect: $3.7 e-09$ Matches: 17

Hemopexin (Fragnent).- Mus musculus (Mouse). 


\section{Protein View}

Match to: Q3UKP2_MOUSE score: 211 Expect: $7.3 e-17$

14 days embryo liver cDNA, RIKEN full-length enriched library, clone:I530016F13 product:hemopexin Found in search of pmf_o2_129990849114.txt

Nom1nal mass $\left(M_{T}\right): 51285$; Calculated pI value: 7.92

NCBI BLAST search of Q3UKP2 MOUSE aga1nst nr

unformatted sequence string for pasting 1nto other applications

Taxonorny: Mus musculus

Links to retrieve other entries conta1ning this sequence from NCBI Entrez:

(no taxonomy information for this entry)

Var1able mod1f1cat1ons: Carban1domethyl (C), Dean1dated (NQ), ox1dat1on (M)

cleavage by Trypsin: cuts c-term side of KR unless next residue $1 \mathrm{~s} F$

Number of mass values searched: 111

Number of mass values matched: 29

sequence coverage: $58 \%$

Matched peptides shown in Bold Red

1 MARTAVALNI LVLLGLCWSL AVASPLPTAN GRVAEVENGT KPDSDVPEHC

51 LDTWSFDAAT MDHNGTMLFF KGEFVWRGHS GTRELISAFW KNPITSVDAA

101 FRGPDSVFLI KEDKVWVYPP EKKENGYPKL FQEEFPGIPY PPDAAVECHR

151 GECQSEGVLF FQGNRKWFWD FATRTQKERS WSTVGNCTAA LRWLERYYCF

201 QGNKFLRFNP VTGEVPPRYP LDARDYEVSC PGRGHGRPRN GTAHGNSTHP

251 MHSRCSPDPG LTALLSDHRG ATYAFTGSHY WRLDSSRDGW HSWPIAHHWP

301 QGPSTVDAAF SWDDKVYLIQ GTQVYVELTK GGNNLVSGYP KRLEKELGSP

351 PGISLETIDA AFSCPGSSRL YVSSGRRLWW LDLKSGAQAT WTEVSWPHEK

401 VDGALCLDKS LGPNTCSSNG SSLYFIHGPN LYCYSSIDKL NAAKSLPQPQ

451 KVNSILGCSQ

Show predicted peptides also

\section{Sort Peptides By}

Start - End
$90-102$
$92-102$
$103-111$
$103-114$
$115-122$
$115-123$
$130-150$

- Residue Number

Obse rved
1504.8770
1190.6782
975.5938
1347.7842
1017.5829
1145.6919
2472.3220

\section{Mr (expt)}

1503.8697

1189.6709

974.5865

1346.7769

1016.5756

1144.6846

2471.3147
Increasing Mass

Decreasing Mass

$M r(c a l c)$
1503.7834
1189.6091
974.5437
1346.7082
1016.5331
1144.6281
2471.1580

ppm
57
52
44
51
42
49
63

\section{Miss Sequence}

1 R. WKNPITSVDAAFR, G

O K. NPITSVDAAFR. G

o R. GPDSVFLIK. E

1 R. GPDSVFLIKEDK.V

O K. VWVYPPEK. K

1 K. VWVYPPEKK. E

0 K. LFQEEFPGIPYPPDAAVECHR.G carh 


\section{M15}

$4700 \mathrm{MS} / \mathrm{MS}$ Precursor 1051.75 Spec \#1 MC[BP = 861.0, 3680]

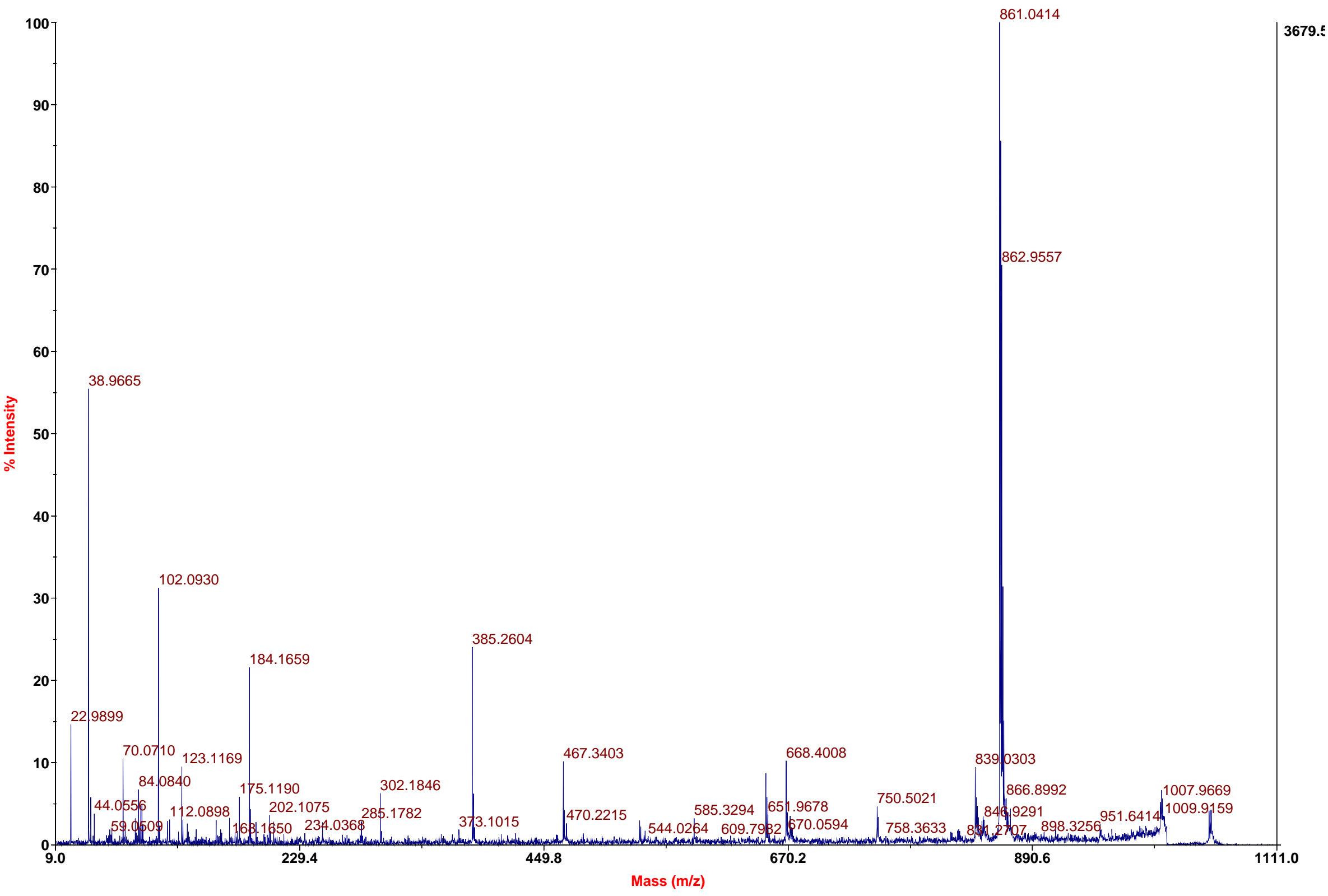




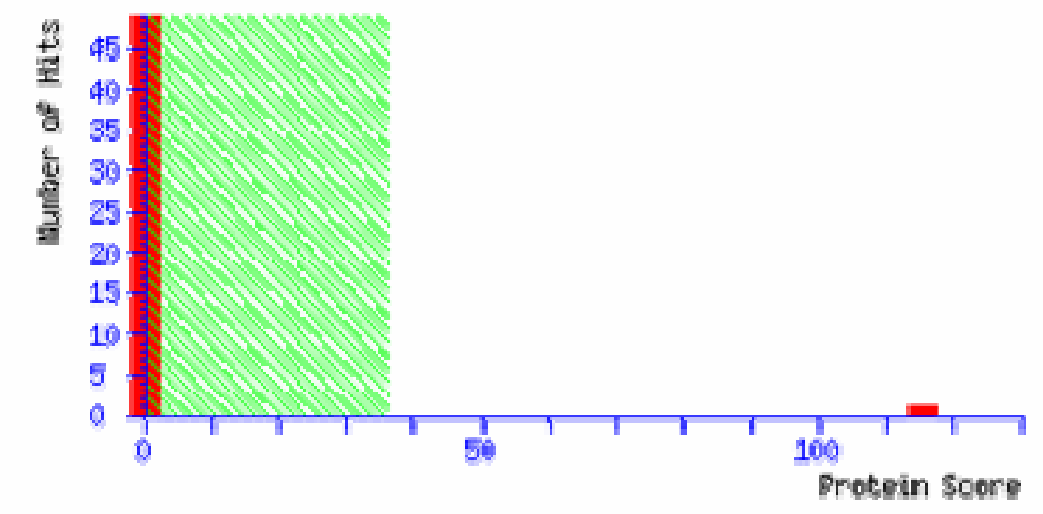

\section{Peptide Summary Report}

Format As Peptide Summary

Significance threshold $\mathrm{p}<0.05$

Standard scoring MudPIT scoring $\odot$ Ions score or expect cut-off 0

Show pop-ups 9 Suppress pop-ups 9 Sort unassigned Decreasing Score $\underline{\text { Help }}$

Show sub-sets 0

Require bold red

\section{$\square$ Error tolerant}

1. Q3UKP2_MOUSE Mass: 51285 Score: 115 Matches: $14(3)$ Sequences: $14(3)$ emPAI: 0.95

14 days embryo l1ver cDNA, RIKEN full-length enrlched 11brary, clone:I530016F13 product:henopex1n, full 1nsert sed

$\checkmark$ check to 1nclude this hit in error tolerant search

\begin{tabular}{rrrrrrrr}
\multicolumn{1}{r}{ Query } & Observed & Mr(expt) & Mr(calc) & ppm & Miss & Score \\
& $\frac{2}{3}$ & 975.5938 & 974.5865 & 974.5437 & 43.9 & 0 & 25 \\
$\square$ & $\frac{3}{6}$ & 1017.5829 & 1016.5756 & 1016.5331 & 41.8 & 0 & 14 \\
$\square$ & $\frac{6}{7}$ & 1079.5121 & 1078.5048 & 1078.4542 & 46.9 & 0 & 25 \\
$\square$ & $\underline{7}$ & 1100.5388 & 1099.5315 & 1099.4757 & 50.8 & 0 & 22 \\
$\square$ & $\underline{8}$ & 1128.5817 & 1127.5744 & 1127.5189 & 49.3 & 0 & 4 \\
$\square$ & $\underline{9}$ & 1145.6919 & 1144.6846 & 1144.6281 & 49.4 & 1 & 6 \\
$\square$ & $\underline{11}$ & 1190.6782 & 1189.6709 & 1189.6091 & 51.9 & 0 & 31 \\
$\square$ & $\underline{12}$ & 1212.7021 & 1211.6948 & 1211.6299 & 53.6 & 0 & 35 \\
$\square$ & $\underline{14}$ & 1504.8770 & 1503.8697 & 1503.7834 & 57.4 & 1 & 12
\end{tabular}

\section{Expect Rank Unique Peptide}

$0.62 \quad 3 \quad$ U R. GPDSVFLIK.E

$\begin{array}{llll}13 & 3 & \mathrm{U} & \mathrm{K} \text {. VWVYPPEK. K }\end{array}$

$\begin{array}{lll}1.1 & 1 & \mathrm{U}\end{array}$

$\begin{array}{lll}1.8 & 1 & \mathrm{U}\end{array}$

R. YYCFQGNK. F + Carbamidomethyl (C)

R. DYFVSCPGR.G + Carbamidomethyl (C)

K. WFWDFATR. T

$\begin{array}{rrrl}46 & 2 & \text { U } & \text { K. WWVYPPEKK.E } \\ 0.22 & 1 & \text { U } & \text { K. NPITSVDAAFR. G }\end{array}$

$\begin{array}{llll}0.052 & 1 & \mathrm{U} & \text { R. FNPVTGEVPPR.Y }\end{array}$

$\begin{array}{llll}11 & 1 & \mathrm{U} & \text { R. WKNPITSVDAAFR. G }\end{array}$ 


\section{Protein View}

Match to: Q3UKP2_MOUSE score: 115

14 days embryo liver CDNA, RIKEN full-length enriched library, clone:I530016F13 product:hemopexin, full Found 1n search of Ppw_O2_129990856114.txt

Nom1nal mass $\left(M_{x}\right)$ : 51285; Calculated pI value: 7.92

NCBI BLAST search of Q3UKP2_MOUSE aga1nst $\mathrm{nr}$

Unformatted sequence string for pasting into other applications

Taxonony: Mus musculus

Links to retrieve other entries containing this sequence from NCBI Entrez:

(no taxonomy information for this entry)

Var1able modif1cat1ons: Carban1donethyl (C), Dean1dated (NQ), Ox1dation

cleavage by Trypsin: cuts C-term side of KR unless next residue $1 \mathrm{~s} P$

sequence coverage: $33 \%$

Matched peptides shown in Bold Red

1 MARTAVALNI LVLLGLCWSL AVASPLPTAN GRVAEVENGT KPDSDVPEHC

51 LDTWSFDAAT MDHNGTMLFF KGEFVWRGHS GTRELISARW KNPITSVDAA

101 FRGPDSVFLI KEDKVWVYPP EKKENGYPKL FQEEFPGIPY PPDAAVECHR

151 GECQSEGVLF FQGNRKWFWD FATRTQKERS WSTVGNCTAA LRWLERYYCF

201 QGNKFLRFNP VTGEVPPRYP LDARDYFVSC PGRGHGRPRN GTAHGNSTHP

251 MHSRCSPDPG LTALLSDHRG ATYAFTGSHY WRLDSSRDGW HSWPIAHHWP

301 QGPSTVDAAF SWDDKVYLIQ GTQVYVELTK GGNNLVSGYP KRLEKELGSP

351 PGISLETIDA AFSCPGSSRL YVSSGRRLWW LDLKSGAOAT WTEVSWPHEK

401 VDGALCLDKS LGPNTCSSNG SSLYFIHGPN LYCYSSIDKL NAAKSLPQPQ

451 KVNSILGCSQ

Show predicted peptides also

\section{Sort Peptides By}

Start - End
$90-102$
$92-102$
$103-111$
$115-122$
$115-123$
$130-150$
$151-165$
$167-174$
$197-204$

(9) Residue Number

Obse rved
1504.8770
1190.6782
975.5938
1017.5829
1145.6919
2472.3220
1727.8873
1128.5817
1079.5121

\section{Mr (expt)}

1503.8697

1189.6709

974.5865

1016.5756

1144.6846

2471.3147

1726.8800

1127.5744

1078.5048
Increasing Mass

Decreasing Mass

ppm
57
52
44
42
49
63
62
49
47

\section{Miss Sequence}

1 R. WKNPITSVDAAFR.G (Ions score 12)

0 K. NPITSVDAAFR. G (Ions score 31)

R. GPDSVFLIK.E (IONS score 25)

K. VWVYPPEK.K (IOns score 14)

1 K. VWVYPPEKK.E (IOnS score 6)

0 K. LFQEEFPGIPYPPDAAVECHR.G Carban1don R. GECQSEGVLFFQGNR . K Carban1dome thy 1

0 K. WFWDFATR. T (Ions score 4)

o R. YYCFOGNK.F Carbam1domethvi (C) (IC) 
Sample M16

4700 Reflector Spec \#1 MC=>NR(2.00)[BP = 1397.8, 35164]

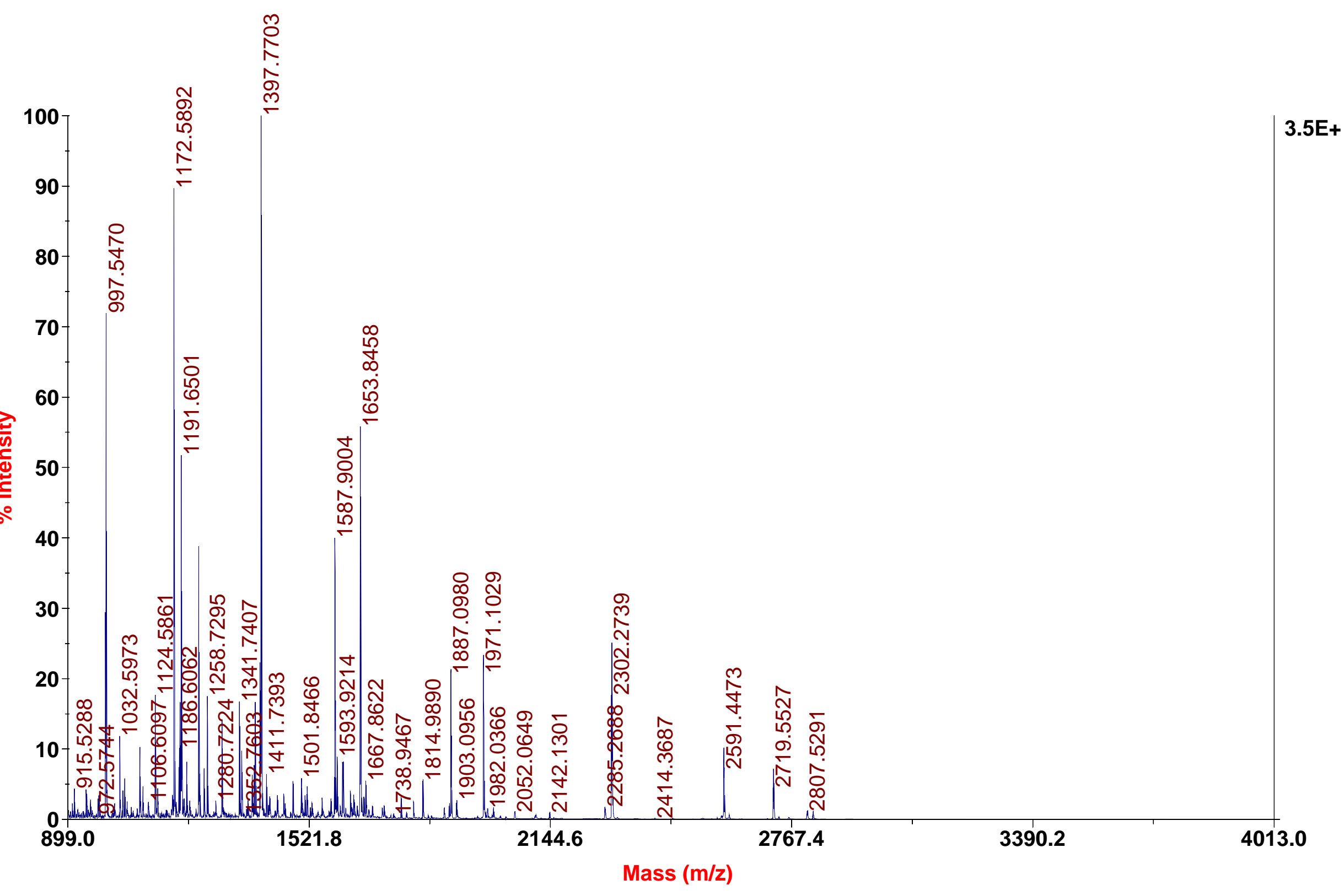




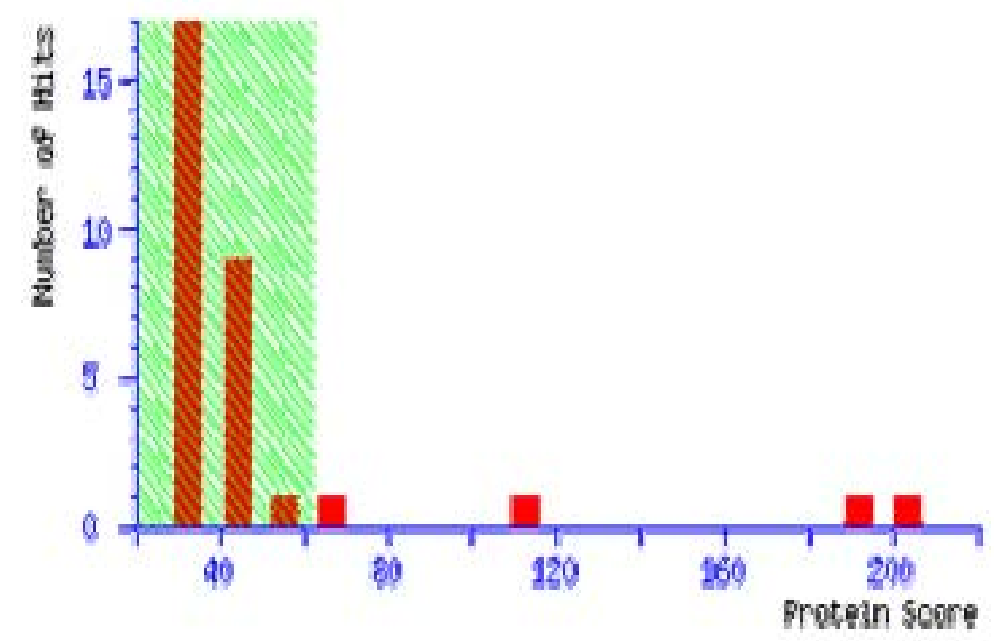

\section{Concise Protein Summary Report}



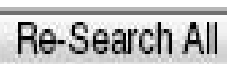

1. OBC2F4 MOUSE Mass: 56590 Score: 203 Expect: $4.6 \mathrm{e}-16$ Matches: 45

2 days neonate thynus thym1c cells CDNM, RIKEN full-length enr1ched l1brary, clone:E430024F21 product:glucose regulated Q99LF6 MOUSE Mass: 56643 Score: 203 Expect: 4.6e-16 Matches: 45 Protein disulfide 1sonerase associated 3.- Mus musculus (Mouse). Q3TEI9 MOUSE Mass: 55393 Score: 190 Expect: 9.2e-15 Matches: 44

2 days neonate thynus thyn1c cells CDNa, RIKEN ful1-length enr1ched 11brary, clone:E430002E15 product:glucose regulated Q3UZK8_MOUSE Mass: 55446 Score: 190 Expect: 9.2e-15 Matches: 44 8 days embryo whole body CDNA, RIKEN full-length enriched library, clone:5730420K02 product:glucose regulated prote1n, AAsa39944 Mass: 56586 Score: 151 Expect: $7.3 \mathrm{e}-11$ Matches: 42 


\section{Protein View}

Match to: Q8C2F4_MOUSE Score: 203 Expect: 4.6e-16

2 days neonate thymus thymic cells cDNA, RIKEN full-length enriched library, clone:E430024F21 product:glucose regulated pr Found 1n search of pmf_P2_129990849115.txt

Nom1nal mass $\left(M_{x}\right)$ : 56590; Calculated pI value: 5.78

NCBI BLAST search of $08 \mathrm{C} 2 \mathrm{~F} 4$ MOUSE aga1nst $\mathrm{nr}$

unformatted sequence string for pasting into other applications

Taxonony: Mus musculus

Links to retrleve other entries containing this sequence from NCBI Entrez:

(no taxonomy information for this entry)

Var1able modifications: Carbanidonethyl (C), Deam1dated (NQ), oxidation (M)

cleavage by Trypsin: cuts C-term side of $\mathrm{KR}$ unless next residue is $\mathrm{F}$

Number of mass values searched: 170

Number of mass values matched: 45

sequence coverage: 698

Matched peptides shown 1n Bold Red

1 MRFSCLALLP GVALLLASAC LAAASDVLEL TDENFESRVS DTGSAGLMLV

51 EFFAPWCGHC KRLAPEYEAA ATRLKGIVPL AKVDCTANTN TCNKYGVSGY 101 PTLKIFRDGE EAGAYDGPRT ADGIVSHLKK QAGPASVPLR TEEEFKKFIS

151 DKDASVVGFF RDLFSDGHSE FLKAASNLRD NYRFAHTNIE SLVKEYDDNG

201 EGITIFRPLH LANKFEDKTV AYTEKKMTSG KIKKFIQDSI FGLCPHMTED

251 NKDLIQGKDL LTAYYDVDYE KNAKGSNYWR NRVMMVAKKF LDAGHKLNEA

301 VASRKTFSHE LSDFGLESTT GEVPVVAIRT AKGEKFVMQE EFSRDGKALE

351 QFLQEYFDGN LKRYLKSEPI PESNEGPVKV VVAENFDDIV NEEDKDVLIE

401 FYAPWCGHCK NLEPKYKELG EKLSKDPNIV IAKMDATAND VPSPYEVKGF

451 PTIYFSPANK KLTPKKYEGG RELNDFISYL QREATNPPII QEEKPKKKKK

501 AQEDL

Show predicted peptides also
Sort Peptides By
(9) Residue Number
Increasing Mass
Decreasing Mass

$\begin{aligned} \text { Start } & - \text { End } \\ 39 & -61 \\ 62 & -73 \\ 63 & -73 \\ 95 & -104 \\ 95 & -107 \\ 105 & -119 \\ 108 & -119 \\ 120 & -129 \\ 120 & -130 \\ 130 & -140\end{aligned}$

$\begin{array}{ccc}\text { Observed } & \text { Mr(expt) } & \text { Mr (calc) } \\ 2585.3071 & 2584.2998 & 2584.1549 \\ 1347.7603 & 1346.7530 & 1346.6942 \\ 1191.6493 & 1190.6420 & 1190.5931 \\ 1084.6094 & 1083.6021 & 1083.5601 \\ 1500.7830 & 1499.7757 & 1499.8136 \\ 1652.8489 & 1651.8416 & 1651.7590 \\ 1236.5663 & 1235.5590 & 1235.5054 \\ 1040.6093 & 1039.6020 & 1039.5662 \\ 1168.7041 & 1167.6968 & 1167.6612 \\ 1123.6980 & 1122.6907 & 1122.6509\end{array}$

ppm
56
44
41
39
-25
50
43
34
31
35

Miss Sequence

0 R. VSDTGSAGLMLVEFFAPWCGHCK.R 2 Carbamidomethyl (C); oxid

1 K. RLAPEYEAAATR . L

0 R. LAPEYEAAATR. L

0 K. YGVSGYPTLK. I

1 K. YGVSGYPTLKIFR. D

1 K. IFRDGEEAGAYDGPR.T

0 R. DGEEAGAYDGPR. T

0 R. TADGIVSHLK. K

1 R. TADGIVSHLKK. $Q$

1 K. KQA GPASVPLR. T 


\section{M16}

4700 MS/MS Precursor 1397.77 Spec \#1 MC[BP = 86.1, 4255]

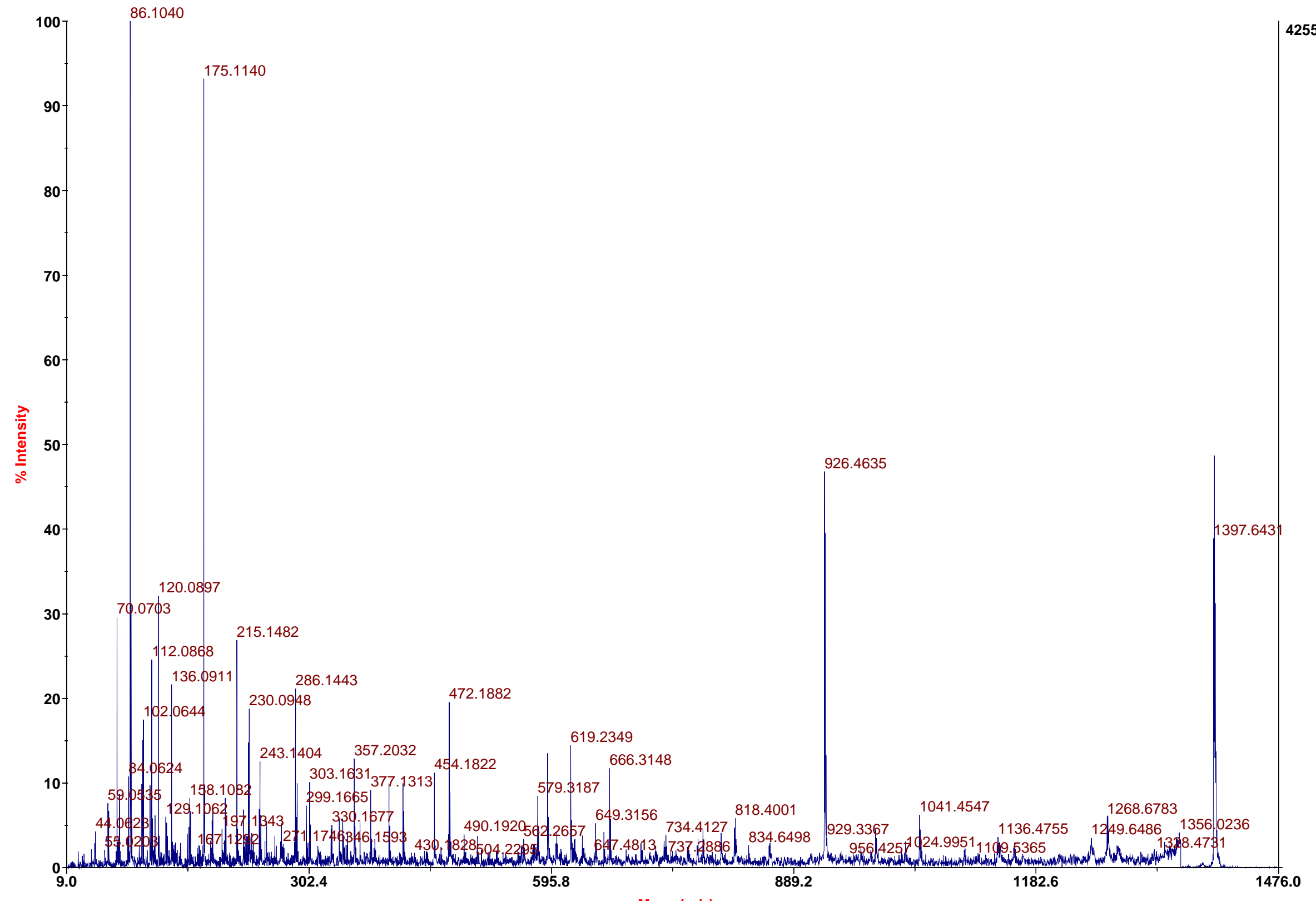

Mass (m/z) 


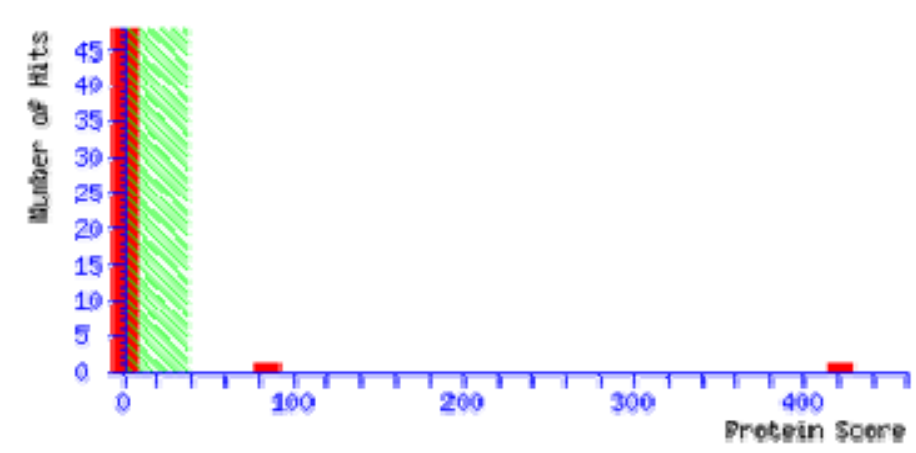

\section{Peptide Summary Report}

\section{Format As Peptide Summary}

Significance threshold $\mathrm{p}<0.05$

Standard scoring MudPIT scoring Ions score or expect cut-off 0

Show pop-ups Suppress pop-ups Sort unassigned Decreasing Score
Help

Show sub-sets 0

Require bold red $\square$

\section{Select All}

\section{Select None}

Search Selected

\section{$\square$ Error tolerant}

1. 3 TEI9 MOUSE Mass: 55393 Score: 421 Matches: 15 (11) Sequences: 15(11) emPAI: 3.06

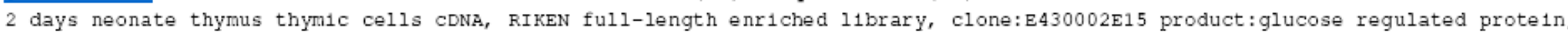
$\square$ check to include this hit in error tolerant search

\begin{tabular}{|c|c|c|c|c|c|c|c|c|c|c|c|}
\hline \multicolumn{2}{|c|}{ Query } & Obse rved & Mr (expt) & $\operatorname{Mr}(\mathrm{calc})$ & ppm & Miss & Score & Expect & Rank & Unique & Peptide \\
\hline 囯 & $\underline{1}$ & 997.5465 & 996.5392 & 996.5029 & 36.5 & 0 & 45 & 0.0075 & 1 & $\mathrm{U}$ & K. DASVVGEFR. D \\
\hline 目 & $\underline{3}$ & 1084.6094 & 1083.6021 & 1083.5601 & 38.8 & 0 & 27 & 0.43 & 1 & $\mathrm{U}$ & K. YGVSGYPTLK. I \\
\hline च & $\underline{5}$ & 1172.5880 & 1171.5807 & 1171.5332 & 40.6 & 0 & 41 & 0.023 & 1 & U & K. FVMQEEFSR.D \\
\hline 可 & $\underline{6}$ & 1191.6493 & 1190.6420 & 1190.5931 & 41.1 & 0 & 43 & 0.014 & 1 & $\mathrm{U}$ & R. LAPEYEAAATR. L \\
\hline 団 & $\underline{7}$ & 1236.5663 & 1235.5590 & 1235.5054 & 43.4 & 0 & 72 & 1. $7 e-05$ & 1 & $\mathrm{U}$ & R. DGEEAGAYDGPR. T \\
\hline 団 & $\underline{9}$ & 1258.7286 & 1257.7213 & 1257.6717 & 39.4 & 0 & 70 & 2. $6 e-05$ & 1 & $\mathrm{U}$ & R. FAHTNIESLVK. E \\
\hline D & $\underline{10}$ & 1341.7379 & 1340.7306 & 1340.6765 & 40.4 & 0 & 29 & 0.32 & 1 & $\mathrm{U}$ & K. GFPTIYESPANK. K \\
\hline 田 & $\underline{11}$ & 1382.7344 & 1381.7271 & 1381.6725 & 39.5 & 0 & 17 & 5.5 & 1 & $\mathrm{U}$ & K. SEPIPESNEGPVK.V \\
\hline 団 & $\underline{12}$ & 1397.7692 & 1396.7619 & 1396.6987 & 45.3 & 0 & 58 & 0.0004 & 1 & $\mathrm{U}$ & R. ELNDF ISYLQR. E \\
\hline 目 & $\underline{13}$ & 1587.8975 & 1586.8902 & 1586.8093 & 51.0 & 1 & 59 & 0.00029 & 1 & $\mathrm{U}$ & K. FISDKDASVVGFER. D \\
\hline 団 & $\underline{14}$ & 1607.8287 & 1606.8214 & 1606.7403 & 50.5 & 0 & 32 & 0.22 & 1 & U & K. DLLTAYYDVDYEK . N \\
\hline 目 & $\underline{29}$ & 1971.0990 & 1970.0917 & 1969.9897 & 51.8 & 1 & 68 & 4. $4 e-05$ & 1 & $\mathrm{U}$ & K. ALEQFLQEYFDGNLKR . Y \\
\hline D & $\underline{50}$ & 2302.2703 & 2301.2630 & 2301.1389 & 53.9 & 0 & 75 & $7 e-06$ & 1 & $\mathrm{U}$ & K. EYDDNGEGITIFRPLHLANK. F \\
\hline 目 & 74 & 2591.4419 & 2590.4346 & 2590.2915 & 55.2 & 0 & 98 & 3. $5 e-08$ & 1 & $\mathrm{U}$ & K. TFSHELSDF GLESTTGEVPVVAIR . T \\
\hline 回 & $\underline{79}$ & 2719.5447 & 2718.5374 & 2718.3865 & 55.5 & 1 & 79 & 2. $4 e-06$ & 1 & $\mathrm{U}$ & R. KTFSHELSDFGLESTTGEVPVVAIR. T \\
\hline
\end{tabular}




\section{Protein View}

Match to: Q3TEI9_MOUSE score: 421

2 days neonate thymus thymic cells cDNA, RIKEN full-length enriched library, clone:E430002E15 product:gl Found 1n search of Ppw_P2_129990856215.txt

Nominal mass $\left(M_{\mathrm{r}}\right)$ : 55393; Calculated pI value: 5.53

NCBI BLAST search of Q3TEI9_MOUSE against $\mathrm{nr}$

unformatted sequence str1ng for pasting into other applications

Taxonony: Mus musculus

Links to retrieve other entries containing this sequence from NCBI Entrez:

(no taxonomy information for this entry)

Var1able mod1f1cat1ons: Carban1domethyl (C), Dean1dated (NQ), ox1dation

cleavage by Trypsin: cuts C-term side of $\mathrm{KR}$ unless next residue $1 \mathrm{~s} P$

sequence coverage: 35 \%

Matched peptides shown in Bold Red

1 MRFSCLALLP GVALLLASAC LAAASDVLEL TDENFESRVS DTGSAGLMLV

51 EFFAPWCGHC KRLAPEYEAA ATRLKGIVPL AKVDCTANTN TCNKYGVSGY

101 PTLKIFRDGE EAGAYDGPRT ADGIVSHLKK QAGPASVPLR TEEEFKKFIS

151 DKDASVVGFF RDLFSDGHSE FLKA.SNLRD NYRFAHTNIE SLVKEYDDNG

201 EGITIFRPLH LANKFEDKTV AYTEKKMTSG KIKKFIODSI FGLCPHMTED

251 NKDLIQGKDL LTAYYDVDYE KNAKGSNYWR NRVMMVAKKF LDAGHKLNEA

301 VASRKTFSHE LSDFGLESTT GEVPVVAIRT AKGEKFVMQE EFSRDGKALE

351 QFLQEYFDGN LKRYLKSEPI PESNEGPVKV VVAENFDDIV NEEDKDVLIE

401 FYAPWCGHCK NLEPKYKELG EKLSKDPNIV IAKMDATAND VPSPYEVKGF

451 PTIYFSPANK KLTPKKYEGG RELNDFISYL QREATNPPII QEEKP

Show predicted peptides also

\section{Sort Peptides By $\odot$ Residue Number Increasing Mass Decreasing Mass}

\begin{tabular}{|c|c|c|c|c|c|c|c|}
\hline start & - End & Observed & $\operatorname{Mr}($ expt) & $\operatorname{Mr}(\mathrm{calc})$ & ppm & Miss & Sequence \\
\hline 63 & -73 & 1191.6493 & 1190.6420 & 1190.5931 & 41 & 0 & R. LAPEYEAAATR. L \\
\hline 95 & -104 & 1084.6094 & 1083.6021 & 1083.5601 & 39 & 0 & (Ions score 27) \\
\hline 108 & -119 & 1236.5663 & 1235.5590 & 1235.5054 & 43 & 0 & R. DGEEAGAYDGPR. T \\
\hline 148 & -161 & 1587.8975 & 1586.8902 & 1586.8093 & 51 & 1 & K. FISDKDASVVGFFR. D (Ions score 59) \\
\hline
\end{tabular}


Sample M17

4700 Reflector Spec \#1 MC=>NR(2.00)[BP = 953.5, 9563]

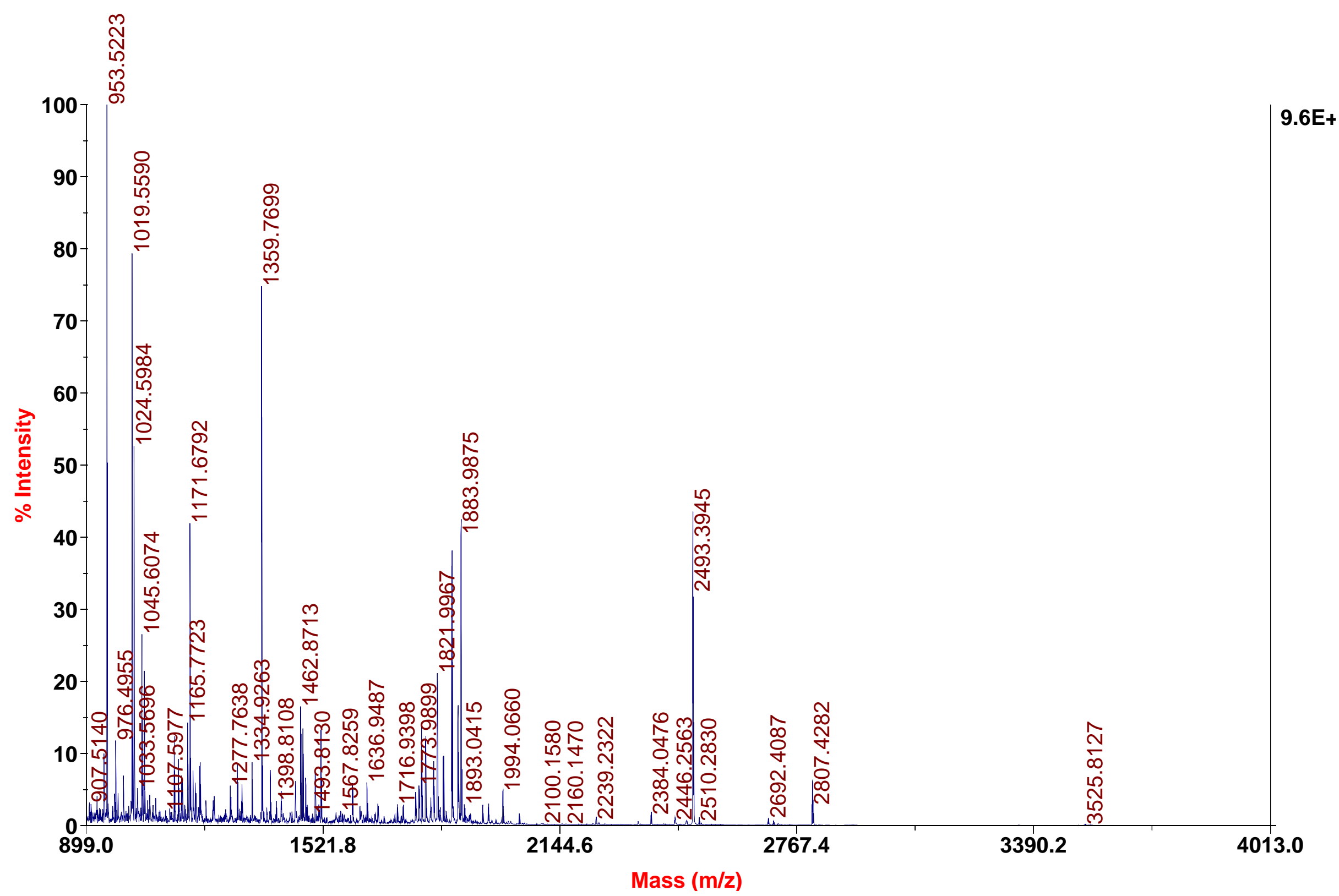




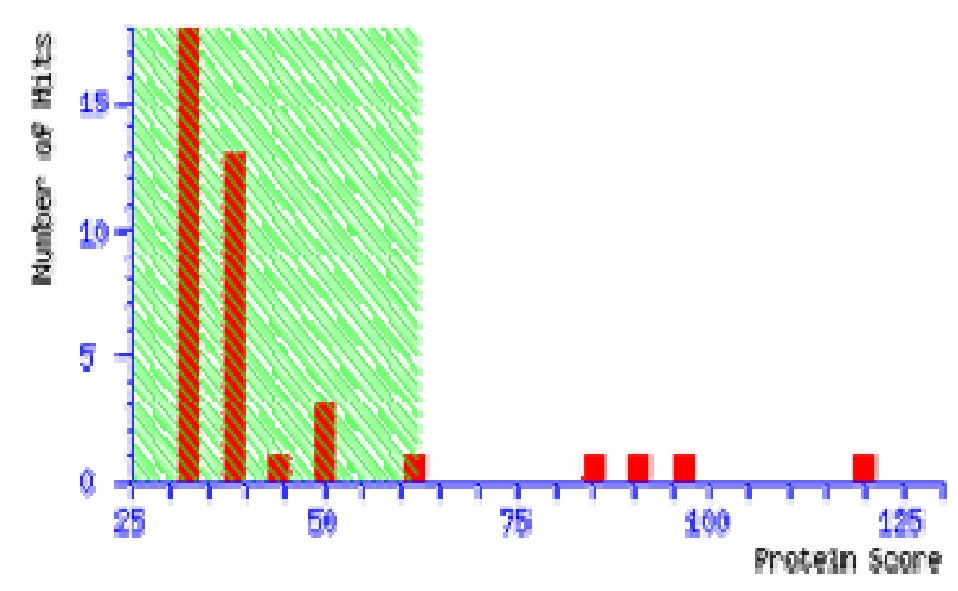

\section{Concise Protein Summary Report}

Format As Concise Protein Summary .

Significance threshold $p<\longdiv { 0 . 0 5 }$ Max. number of hits AUTO

Re-Search All Search Unmatched

1. Q3U1X3 MOUSE Mass: 43138 Score: 120 Expect: $9.2 e-08$ Matches: 35

B6-derived CD11 +ve dendr1t1c cells CDNA, RIKEN full-length enr1ched library, clone:F730110c17 product:pyruvate k1nase, m.

2. Q4VC20_MOUSE Mass: 57808 Score: 99 Expect: $1.1 \mathrm{e}-05$ Matches: 38

Pyruvate kinase 3.- Mus musculus (Mouse).

Q3TBW5_MOUSE Mass: 57807 Score: 99 Expect: $1.1 \mathrm{e}-05$ Matches: 38

NOD-der1ved CD11C +ve dendr1tic cells CDNA, RIKEN full-length enriched l1brary, clone:F630218007 product:pyruvate k1nase, 03TC59 MOUSE Mass: 57809 Score: 99 Expect: $1.1 \mathrm{e}-05$ Matches: 37

NOD-derived CD11c +ve dendritic cells CDNA, RIKEN full-length enriched 11brary, clone:F630207021 product:pyruvate k1nase, 03U5P6 MOUSE Mass: 57820 Score: 99 Expect: 1.1e-05 Matches: 36

Bone marrow macrophage CDNA, RIKEN full-length enriched 11brary, clone: I830166F06 product:pyruvate kinase, nuscle, full 11 Q3TBV8_MOUSE Mass: 57750 Score: 90 Expect: $8.4 e-05$ Matches: 37

NOD-der1ved CD11c +ve dendr1tic cells CDNA, RIKEN full-length enriched l1brary, clone:F630220E24 product:pyruvate k1nase, 


\section{Protein View}

Match to: Q3U1X3_MOUSE Score: 120 Expect: 9.2e-08

B6-derived CD11 +ve dendritic cells cDNA, RIKEN full-length enriched library, clone:F730110C17 product:pyruvate kinase, muscle Found 1n search of pmf_A6_129990866000.txt

Nom1nal mass $\left(M_{\mathrm{r}}\right)$ : 43138; Calculated pI value: 5.88

NCBI BLAST search of Q3U1X3_MOUSE aga1nst nr

Unformatted sequence string for pasting into other applications

Taxonony: Mus musculus

Links to retrieve other entries contalning this sequence from NCBI Entrez:

(no taxonomy information for this entry)

Var1able mod1f1cations: Carbanidonethyl (C), Deanidated (NQ), oxidation (M)

cleavage by Trypsin: cuts C-term side of $\mathrm{KR}$ unless next residue is $\mathrm{P}$

Number of mass values searched: 159

Number of mass values matched: 35

sequence Coverage: 83 .

Matched peptides shown in Bold Red

1 MPKPHSEAGT AFIQTQQLHA AMADTFLEHM CRLDIDSAPI TARNTGIICT

51 IGPASRSVEM LKEMIKSGMN VARLNFSHGT HEYHAETIKN VREATESFAS

101 DPILYRPVAV ALDTKGPEIR TGLIKGSGTA EVELKKGATL KITLDNAYME

151 KCDENILWLD YKNICKVVEV GSKIYVDDGL ISLQVKEKGA DFLVTEVENG

201 GSLGSKKGVN LPGAAVDLPA VSEKDIQDLK FGVEQDVDMV FASFIRKAAD

251 VHEVRKVLGE KGKNIKIISK IENHEGVRRF DEILEASDGI MVARGDLGIE

301 IPAEKVFLAQ KMMIGRCNRA GKPVICATQM LESMIKKPRP TRAEGSDVAN

351 AVLDGADCIM LSGETAKGDY PLEAVRMQHL IAREAEAAMF HRLLF

Show predicted peptides also

Sort Peptides By

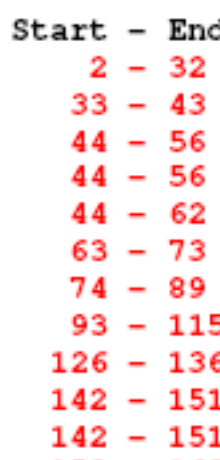

$\begin{array}{rcr}\text { Observed } & \text { Mr(expt) } & \text { Mr(calc) } \\ 3523.7729 & 3522.7656 & 3522.6595 \\ 1171.6792 & 1170.6719 & 1170.6244 \\ 1302.7417 & 1301.7344 & 1301.6762 \\ 1359.7695 & 1358.7622 & 1358.6976 \\ 1990.0870 & 1989.0797 & 1989.0387 \\ 1235.6343 & 1234.6270 & 1234.6162 \\ 1883.9849 & 1882.9776 & 1882.8962 \\ 2493.3906 & 2492.3833 & 2492.2798 \\ 1118.6117 & 1117.6044 & 1117.5979 \\ 1197.6309 & 1196.6236 & 1196.5747 \\ 1213.6306 & 1212.6233 & 1212.5696\end{array}$

Decreasing Mass

$\begin{array}{rclll}\text { Ppm } & \text { Miss } & \text { Sequence } & & \\ 30 & 0 & \text { M. PKPHSEAGTAFIQTQQLHAAMADTFLEHMCR. L } & \text { Carbamidomethyl } & \text { (C) } \\ 41 & 0 & \text { R. LDIDSAPITAR. N } & & \\ 45 & 0 & \text { R. NTGIICTIGPASR.S } & \\ 48 & 0 & \text { R. NTGIICTIGPASR.S Carbamidomethyl (C) } \\ 21 & 1 & \text { R. NTGIICTIGPASRSVEML. E } & \\ 9 & 1 & \text { K. EMIKSGMNVAR. L } \\ 43 & 0 & \text { R. LNFSHGTHEYHAETIK.N } \\ 42 & 0 & \text { R. EATESFASDPILYRPVAVALDTK. G } \\ 6 & 1 & \text { K. GSGTAEVELKK. G } \\ 41 & 0 & \text { K. ITLDNAYMEK.C } \\ 44 & 0 & \text { K. ITLDNAYMEK.C OX1dation (M) }\end{array}$




\section{M17}

4700 MS/MS Precursor 1051.74 Spec \#1 MC[BP = 861.0, 4180]

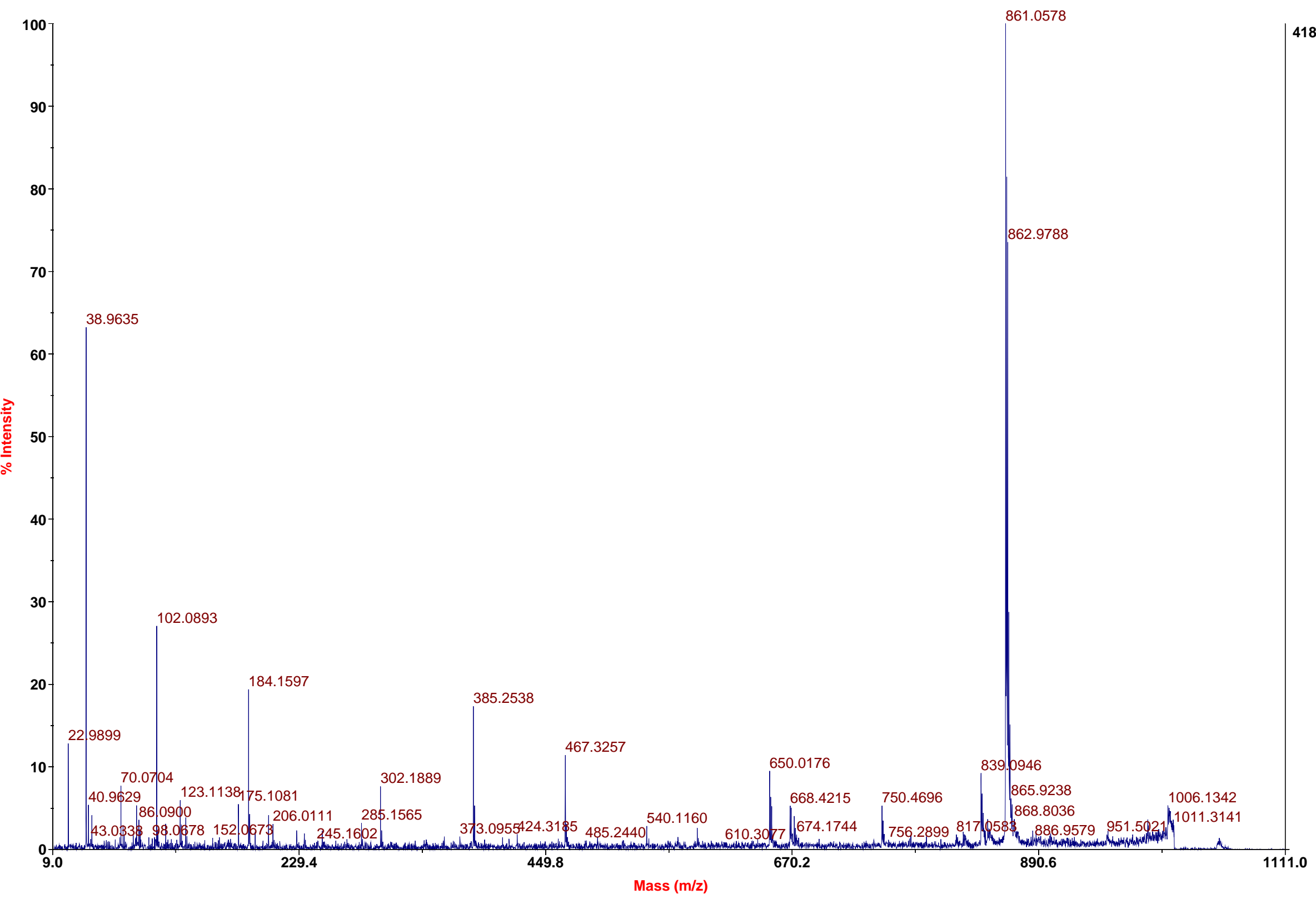




\section{M17}

4700 MS/MS Precursor 976.496 Spec \#1 MC[BP = 343.2, 1047]

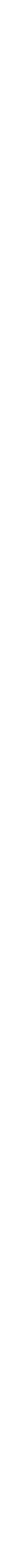




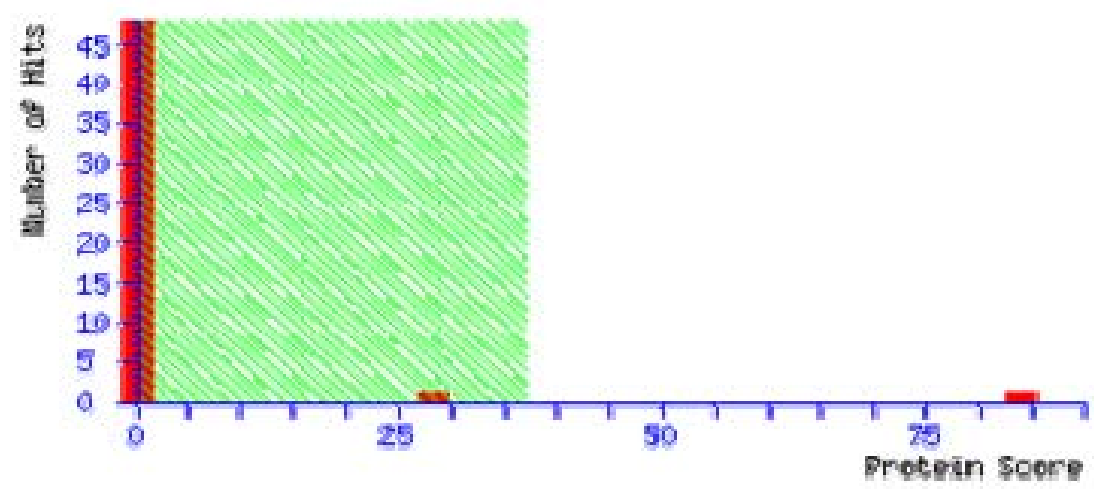

\section{Peptide Summary Report}

\section{Format As Peptide Summary}

Significance threshold $\mathrm{p}<0.05$

Standard scoring MudPIT scoring - Ions score or expect cut-off 0

Show pop-ups (9) Suppress pop-ups Sort unassigned Decreasing Score $\underline{\text { Help }}$

Show sub-sets 0

Require bold red

Select None

Search Selected

\section{$\square$ Error tolerant}

1. KPYM MOUSE Mass: 57719 Score: 84 Matches: 10(3) Sequences: 10 (3) emPAI: 0.98 Pyruvate k1nase 1sozyne M2 (EC 2.7.1.40).- Mus musculus (Mouse).

check to 1 nclude this hit in error tolerant search

\begin{tabular}{|c|c|c|c|c|}
\hline \multicolumn{2}{|c|}{ Query } & Observed & $\operatorname{Mr}(e x p t)$ & $\mathrm{Mr}$ (calc) \\
\hline 四 & 1 & 953.5208 & 952.5135 & 952.4726 \\
\hline \multirow[t]{2}{*}{ 目 } & $\underline{3}$ & 1019.5587 & 1018.5514 & 1018.5083 \\
\hline & 7 & 1141.6431 & 1140.6358 & 1140.6026 \\
\hline 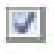 & $\underline{8}$ & 1171.6792 & 1170.6719 & 1170.6244 \\
\hline (D) & $\underline{9}$ & 1359.7695 & 1358.7622 & 1358.6976 \\
\hline$\nabla$ & $\underline{10}$ & 1462.8705 & 1461.8632 & 1461.8079 \\
\hline D & 13 & 1779.9463 & 1778.9390 & 1778.8687 \\
\hline 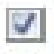 & 18 & 1821.9973 & 1820.9900 & 1820.9091 \\
\hline 同 & 22 & 1859.9863 & 1858.9790 & 1858.8924 \\
\hline 9 & 24 & 1883.9849 & 1882.9776 & 1882.8962 \\
\hline
\end{tabular}

$\begin{array}{lcc}\text { ppm } & \text { Miss } & \text { Score } \\ 42.9 & 0 & 37 \\ 42.3 & 0 & 31 \\ 29.1 & 0 & 22 \\ 40.6 & 0 & 39 \\ 47.5 & 0 & 34 \\ 37.9 & 0 & 34 \\ 39.6 & 0 & 46 \\ 44.5 & 1 & 23 \\ 46.6 & 0 & 16 \\ 43.2 & 0 & 26\end{array}$

Expect Rank Unique 0.037

0.231

1. 43

0.0291

0.111

0.061

0. 00751

1. 61

7.91

$0.72 \quad 1$

\section{Peptide}

K. IENHEGVR. R

K. GDYPLEAVR. M

R. GDLGIEIPAEK.V

R. LDIDSAPITAR. N

R. NTGIIĆTIGPASR.S + Carbamí

K. IYVDDGLISLQVK . E

$\mathrm{K}$. GADFLVTEVENGGSLGSK. $\mathrm{K}$

R. REDEILEASDGIMVAR . G

K. FGVEQDVDMVEASEIR . $K$

R. LNFSHGTHEYHAETIK. N 


\section{Protein View}

Match to: KPYM_MOUSE score: 84

Pyruvate kinase isozyme M2 (EC 2.7.1.40).- Mus musculus (Mouse).

Found 1n search of Ppw_A6_129990871300.txt

Nom1nal mass $\left(M_{x}\right)$ : 57719; Calculated $p I$ value: 7.42

NCBI BLAST search of KPYM MOUSE aga1nst nr

unformatted sequence string for pasting 1nto other applications

Taxonony: Mus musculus

Var1able mod1f1cat1ons: Carban1domethyl (C), Dean1dated (NQ), ox1dation cleavage by Trypsin: cuts C-term side of KR unless next residue $15 \mathrm{P}$ sequence coverage: 248

Matched peptides shown 1n Bold Red

1 PKPHSEAGTA FIQTQQLHAA MADTFLEHMC RLDIDSAPIT ARNTGIICTI

51 GPASRSVEML KEMIKSGMNV ARLNFSHGTH EYHAETIKNV REATESFASD

101 PILYRPVAVA LDTKGPEIRT GLIKGSGTAE VELKKGATLK ITLDNAYMEK

151 CDENILWLDY KNICKVVEVG SKIYVDDGLI SLQVKEKGAD FLVTEVENGG

201 SLGSKKGVNL PGAAVDLPAV SEKDIQDLKF GVEQDVDMVF ASFIRKAADV

251 HEVRKVLGEK GKNIKIISKI ENHEGVRRFD EILEASDGIM VARGDLGIEI

301 PAEKVFLAQK MMIGRCNRAG KPVICSTQML EIMIKKPRPT RAEGSDVANA

351 VLDGADCIML SGETAKGDYP LEAVRMOHLI AREAEAAIYH LOLFEELRRL

401 APITSDPTEA AAVGAVEASF KCCSGAIIVL TKSGRSAHQV ARYRPRAPII

451 AVTRNPQTAR QAHLYRGIFP VLCKDAVLNA WAEDVDLRVN LAMDVGKARG

501 FFKKGDVVIV LTGWRPGSGF TNTMRVVPVP

\section{Show predicted peptides also}

\section{Sort Peptides By}

$\begin{aligned} \text { Start } & - \text { End } \\ 32 & -42 \\ 43 & -55 \\ 73 & -88 \\ 173 & -185 \\ 188 & -205 \\ 230 & -245 \\ 270 & -277 \\ 278 & -293 \\ 294 & -304 \\ 367 & -375\end{aligned}$

\section{Obse rved}

1171.6792

1359.7695

1883.9849

1462.8705

1779.9463

1859.9863

953.5208

1821. 9973

1141. 6431

1019.5587
Mr (expt) Mr (calc)

$1170.6719 \quad 1170.6244$

$1358.7622 \quad 1358.6976$

$1882.9776 \quad 1882.8962$

$1461.8632 \quad 1461.8079$

$1778.9390 \quad 1778.8687$

$1858.9790 \quad 1858.8924$

$952.5135 \quad 952.4726$

$1820.9900 \quad 1820.9091$

$1140.6358 \quad 1140.6026$

1018.5514

\author{
(M)
}


Sample M18

4700 Reflector Spec \#1 MC=>NR(2.00)[BP = 1032.6, 17735]

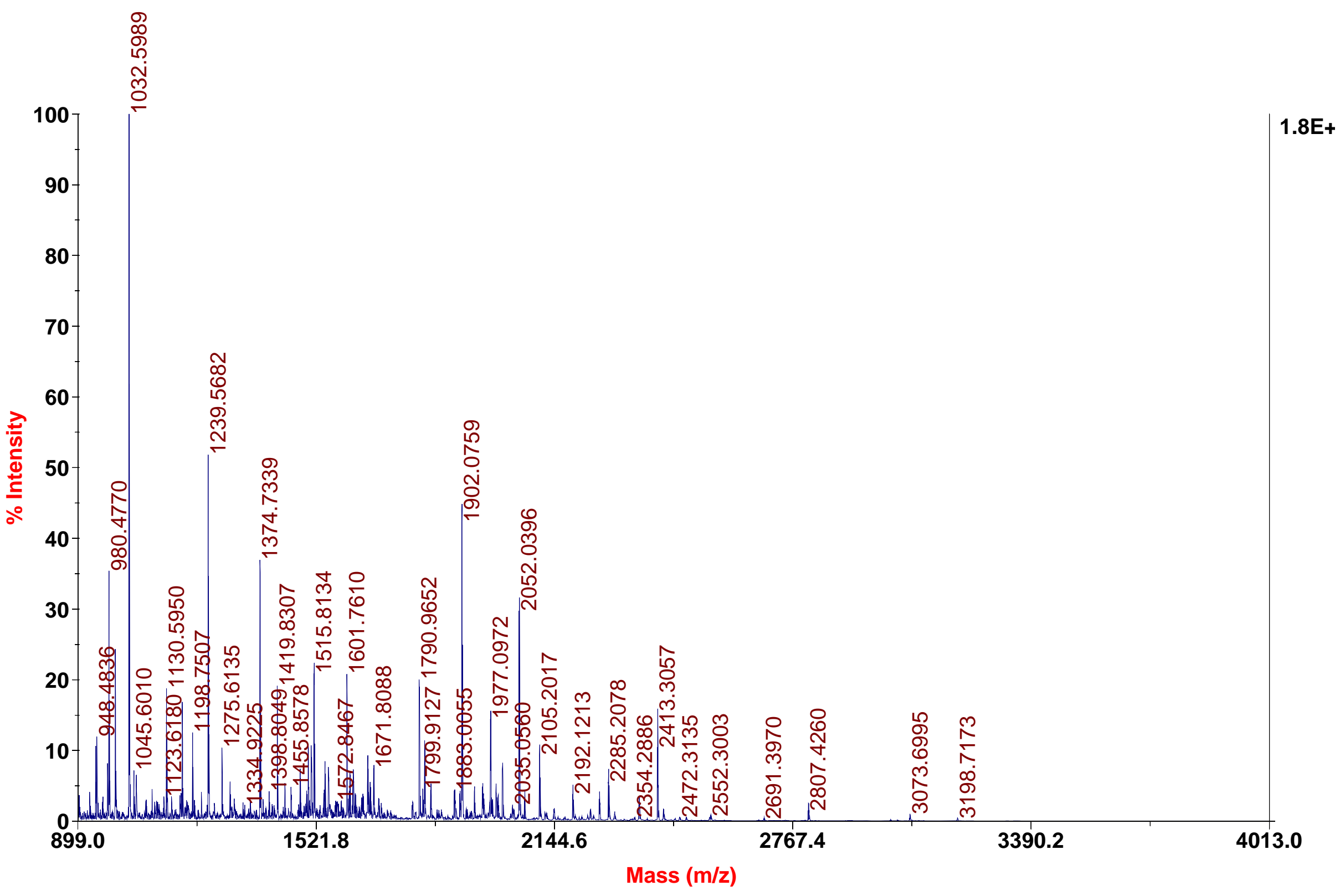




\section{Protein View}

Match to: Q3TGR2_MOUSE score: 110 Expect: 9.2e-07 17 days pregnant adult female amnion cDNA, RIKEN full-length enriched library, clone:I920039H17 product: fibrinogen Found 1n search of pmf_B6_129990866001.txt

Nom1nal mass $\left(M_{x}\right)$ : 54718; Calculated pI value: 6.68

NCBI BLAST search of Q3TGR2_MOUSE aga1nst $\mathrm{nr}$

unformatted sequence string for pasting 1nto other applications

Taxonorny: Mus musculus

Links to retrieve other entries containing this sequence from NCBI Entrez:

(no taxonomy information for this entry)

(no taxonomy information for this entry)

(no taxonomy information for this entry)

(no taxonomy information for this entry)

Var1able mod1f1cat1ons: Carban1donethy 1 (C), Dean1dated (NQ), ox1dation (M)

cleavage by Trypsin: cuts C-term side of $\mathrm{KR}$ unless next residue $1 \mathrm{~s} \mathrm{P}$

Number of mass values searched: 182

Number of mass values matched: $\mathbf{4 2}$

sequence coverage: 668

Matched peptides shown in Bold Red

1 MRHLWLLLLL CVFSVQTQAA DDDYDEFTDS LDARGHRPVD RRKEEPPSLR

51 PAPPPISGGG YRARPAKATA NQKKVERRPP DAGGCLHADT DMGVLCPTGC

101 TLQQTLLNQE RPIKSSIAEL NNNIQSVSDT SSVTFQYLTL LKDMWKKKQA

151 QVKENENVIN EYSSILEDQR LYIDETVNDN IPLNLRVLRS ILEDLRSKIQ

201 KLESDISAQM EYCRTPCTVS CNIPVVSGKE CEEIIRKGGE TSEMYLIQPD

251 TSIKPYRVYC DMKTENGGWT VIQNRQDGSV DFGRKWDFYK KGEGNIATNE

301 DAKKYCGLPG EYWLGNDKIS OLTRMGPTEL LIEMEDWKGD KVKAHYGGET

351 VQNEASKYQV SVNKYKGTAG NALMDGASQL VGENRTMTIH NGMFFSTYDR

401 DNDGWVTTDP RKQCSKEDGG GWWYNRCHAA NPNGRYYWGG LYSWDMSKHG

451 TDDGVVWMNW KGSWYSMRRM SMKIRPFFPQ Q

Show predicted peptides also

\section{Sort Peptides By Residue Number $\odot$ Increasing Mass Decreasing Mass}

$\begin{array}{rrrr}\text { Start - End } & \text { Observed } & \text { Mr(expt) } & \text { Mr (calc) } \\ 43-62 & 2105.2007 & 2104.1934 & 2104.1065 \\ 44-62 & 1977.0981 & 1976.0908 & 1976.0116 \\ 115-142 & 3072.7024 & 3071.6951 & 3071.5663 \\ 154-170 & 2052.0364 & 2051.0291 & 2050.9443 \\ 171-186 & 1902.0759 & 1901.0686 & 1900.9894 \\ 199-214 & 1971.0605 & 1970.0532 & 1969.9237 \\ 202-214 & 1601.7582 & 1600.7509 & 1600.6861\end{array}$

\begin{tabular}{|c|c|c|}
\hline $\mathrm{m}$ & Miss & Sequence \\
\hline 41 & 1 & R. KEEPPSLRPAPPPISGGGYR. A \\
\hline 40 & 0 & K. EEPPSLRPAPPPISGGGYR. A \\
\hline 42 & 0 & K. SSIAELNNNIQSVSDTSSVTFQYLTLLK. D \\
\hline 41 & 0 & K. ENENVINEYSSILEDQR. L \\
\hline 42 & 0 & R. LYIDETVNDNI PLNLR.V \\
\hline 66 & 1 & K. IQKLESDI SAQMEYCR. T \\
\hline 40 & 0 & K. LESDISAQMEYCR. T Carbamidomethy 1 \\
\hline
\end{tabular}




\section{M18}

4700 MS/MS Precursor 2052.04 Spec \#1 MC[BP = 303.1, 3349]

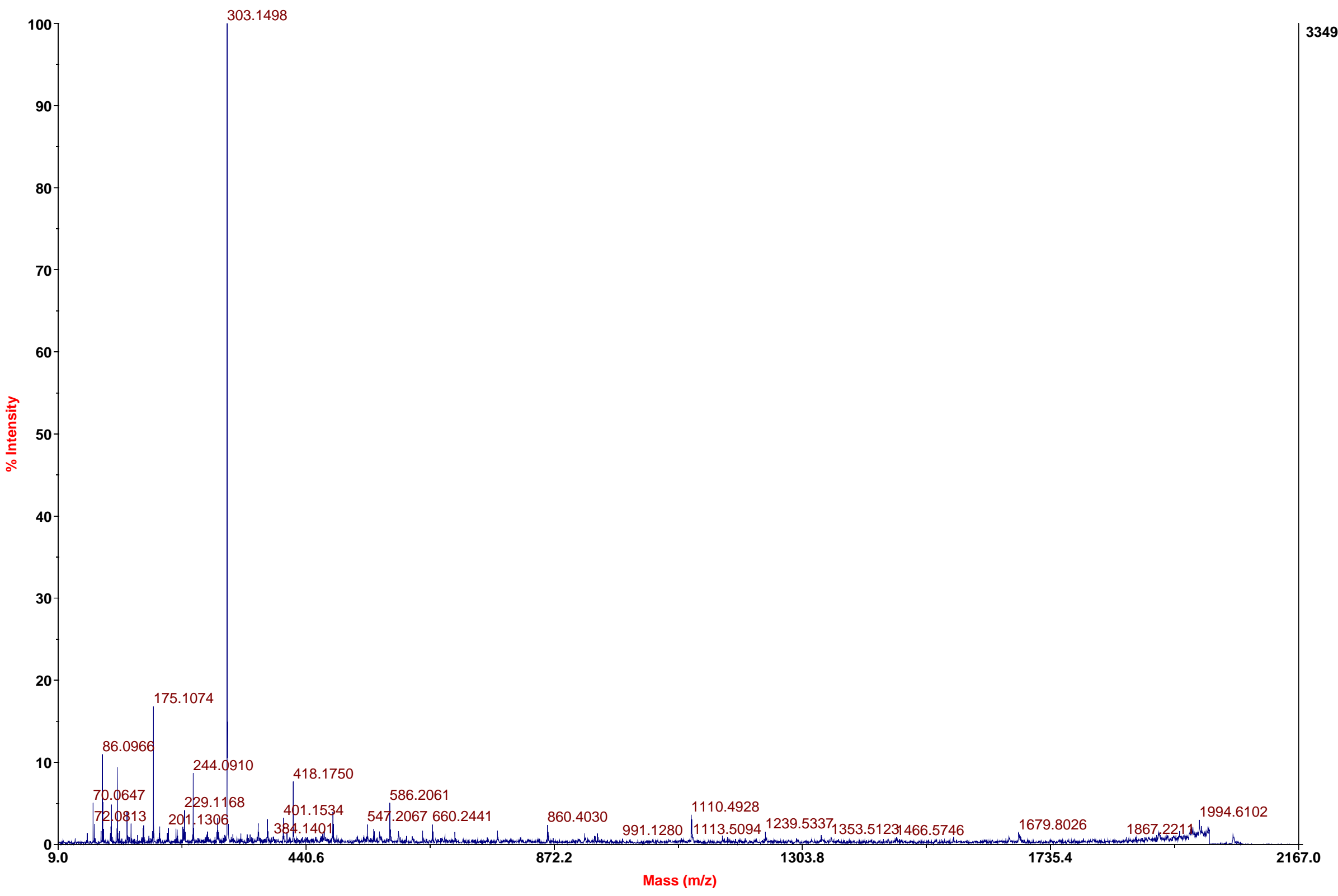




\section{M18}

4700 MS/MS Precursor 1419.83 Spec \#1 MC[BP = 110.1, 1416]

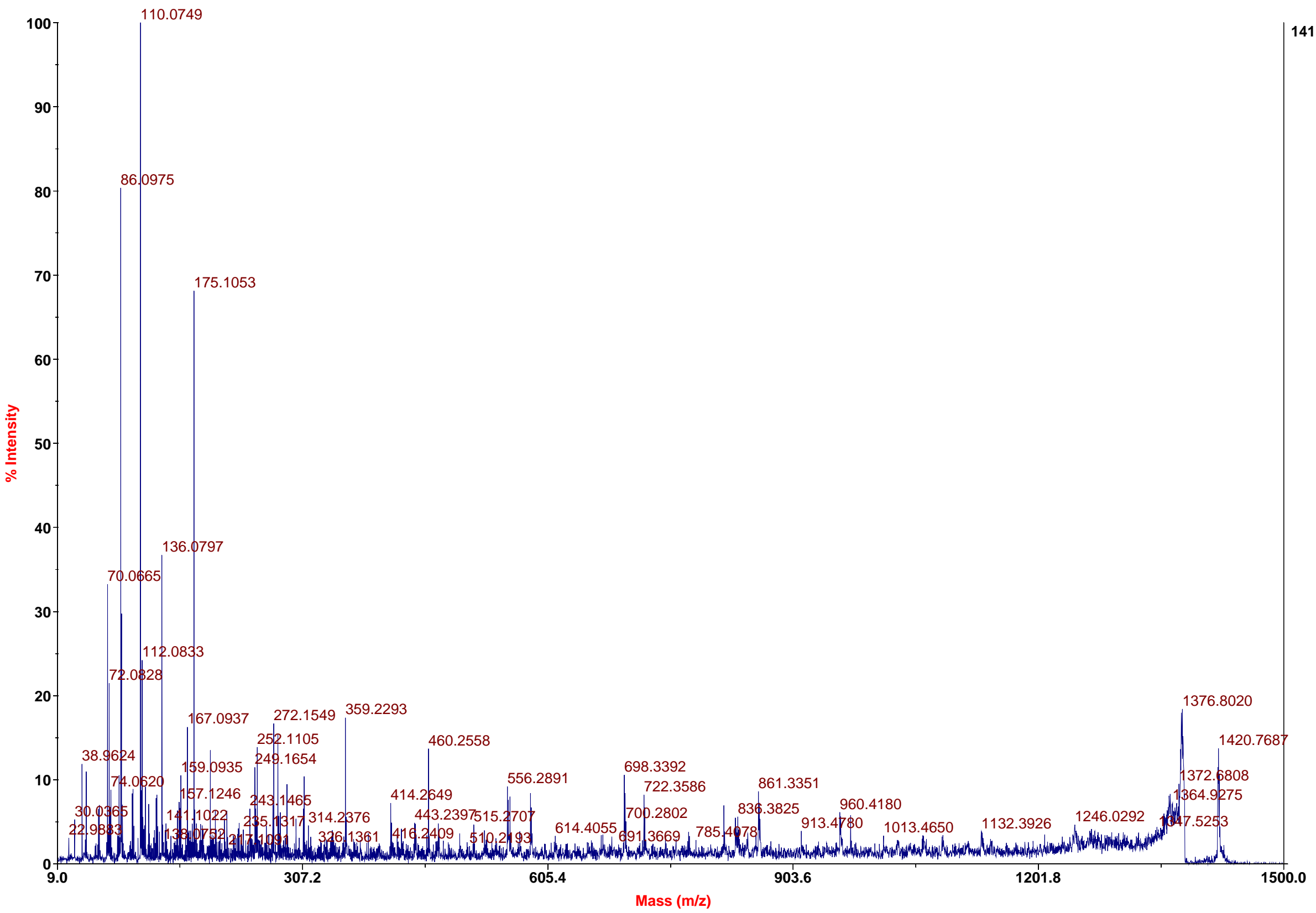




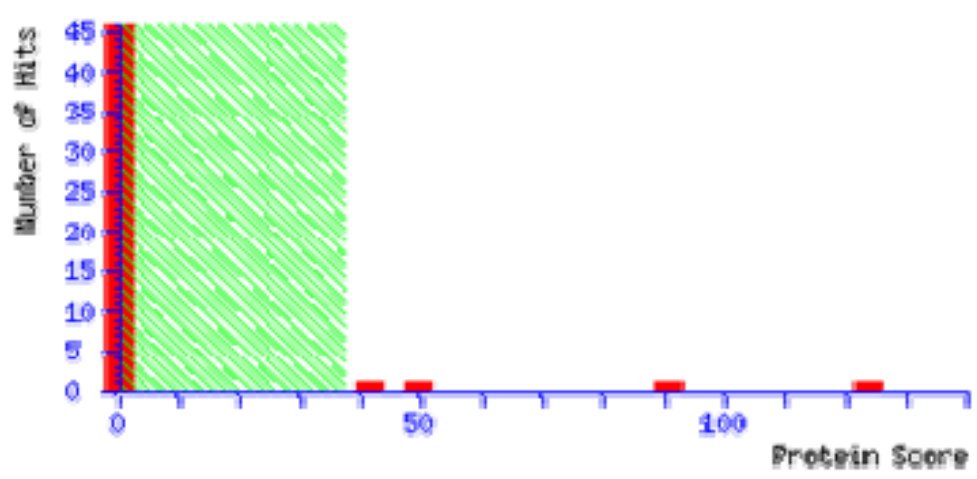

\section{Peptide Summary Report}

Format As Peptide Summary

Significance threshold $\mathrm{p}<0.05$

Standard scoring $\bigcirc$ MudPIT scoring - Ions score or expect cut-off 0

Show pop-ups Suppress pop-ups Sort unassigned Decreasing Score $\underline{\text { Help }}$

Show sub-sets 0

Require bold red

\section{$\square$ Error tolerant}

1. Q3TGR2 MOUSE Mass: 54718 Score: 124 Matches: 12(3) Sequences: 12(3) emPAI: 0.68

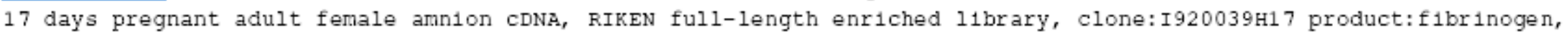

$\square$ check to include this hit in error tolerant search

\begin{tabular}{|c|c|c|c|c|}
\hline \multicolumn{2}{|c|}{ Query } & Observed & $\operatorname{Mr}(e x p t)$ & $\operatorname{Mr}(\mathrm{calc})$ \\
\hline$\nabla$ & $\underline{1}$ & 980.4769 & 979.4697 & 979.4359 \\
\hline 目 & $\underline{2}$ & 996.4788 & 995.4715 & 995.4355 \\
\hline$\nabla$ & $\underline{3}$ & 1032.5969 & 1031.5896 & 1031.5553 \\
\hline & $\underline{7}$ & 1239.5681 & 1238.5608 & 1238.5105 \\
\hline$\nabla$ & $\underline{8}$ & 1374.7316 & 1373.7243 & 1373.6688 \\
\hline వ & $\underline{12}$ & 1601.7582 & 1600.7509 & 1600.6861 \\
\hline$\nabla$ & $\underline{13}$ & 1671.8080 & 1670.8007 & 1670.7399 \\
\hline 目 & $\underline{23}$ & 1902.0759 & 1901.0686 & 1900.9894 \\
\hline 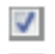 & $\underline{29}$ & 1977.0981 & 1976.0908 & 1976.0116 \\
\hline D & $\underline{32}$ & 2052.0364 & 2051.0291 & 2050.9443 \\
\hline D & $\underline{36}$ & 2105.2007 & 2104.1934 & 2104.1065 \\
\hline D & $\underline{57}$ & 2413.3040 & 2412.2967 & 2412.1995 \\
\hline
\end{tabular}

$\begin{array}{lrrrcr}\text { ppm } & \text { Miss } & \text { Score } & \text { Expect } & \text { Rank Unique } \\ 34.4 & 0 & 27 & 0.52 & 1 & \text { U } \\ 36.2 & 0 & 33 & 0.11 & 1 & \text { U } \\ 33.3 & 0 & 18 & 5 & 1 & \mathrm{U} \\ 40.6 & 0 & 6 & 65 & 4 & \mathrm{U} \\ 40.4 & 0 & 22 & 2.2 & 1 & \mathrm{U} \\ 40.5 & 0 & 15 & 9.6 & 1 & \mathrm{U} \\ 36.4 & 0 & 15 & 11 & 1 & \mathrm{U} \\ 41.7 & 0 & 42 & 0.015 & 1 & \mathrm{U} \\ 40.1 & 0 & 17 & 6.1 & 1 & \mathrm{U} \\ 41.4 & 0 & 51 & 0.0023 & 1 & \mathrm{U} \\ 41.3 & 1 & 19 & 2.6 & 1 & \mathrm{U} \\ 40.3 & 1 & 70 & 2.6 e-05 & 1 & \mathrm{U}\end{array}$

\section{Peptide}

R. QDGSVDFGR . K

R. CHAANPNGR $. Y+$ Carbamidomethyl (C)

K. IRPFFPQQ.-

K. EDGGGWWYNR . C

K. TENGGWTVIQNR . Q

K. LESDI SAQMEYCR.T + Carbamidomethyl

K. YGGLPGEYWLGNDK. I + Carbamidomethyl

R. LYIDETVNDNIPLNLR.V

K. EEPPSLRPAPPPISGGGYR . A

K. ENENVINEYSSILEDQR. L

R. KEEPPSLRPAPPP ISGGGYR. A

R. KGGETSEMYLIQPDTS IKPYR . V 


\section{Protein View}

Match to: Q3TGR2_MOUSE score: 124

17 days pregnant adult female amnion CDNA, RIKEN full-length enriched library, clone: I920039H17 product: fibrinogen, B beta Found 1n search of Ppw_B6_129990871401.txt

Nom1nal mass $\left(\mathrm{M}_{\mathrm{r}}\right)$ : 54718; Calculated pI value: 6.68

NCBI BLAST search of Q3TGR2_MOUSE aga1nst $\mathrm{nr}$

unformatted sequence string for pasting into other applications

Taxonony: Mus musculus

Links to retrieve other entries containing this sequence from NCBI Entrez:

(no taxonomy information for this entry)

(no taxonomy information for this entry)

(no taxonomy information for this entry)

(no taxonomy information for this entry)

Var1able modif1cat1ons: Carbanidonethy 1 (C), Dean1dated (NQ), ox1dation (M) cleavage by Trypsin: cuts c-term side of $\mathrm{KR}$ unless next residue is $\mathrm{P}$

sequence coverage: 308

Matched peptides shown 1n Bold Red

1 MRHLWLLLLL CVFSVQTQAA DDDYDEPTDS LDARGHRPVD RRKEEPPSLR

51 PAPPPISGGG YRARPAKATA NQKKVERRPP DAGGCLHADT DMGVLCPTGC

101 TLQQTLLNQE RPIKSSIAEL NNNIQSVSDT SSVTFQYLTL LKDMWKKKQA

151 QVKENENVIN EYSSILEDQR LYIDETVNDN IPLNLRVLRS ILEDLRSKIQ

201 KLESDISAOM EYCRTPCTVS CNIPVVSGKE CEEIIRKGGE TSEMYLIOPD

251 TSIKPYRVYC DMKTENGGWT VIQNRQDGSV DFGRKWDPYK KGEGNIATNE

301 DAKKYCGLPG EYWLGNDKIS QLTRMGPTEL LIEMEDWKGD KVKAHYGGET

351 VONEASKYQV SVNKYKGTAG NALMDGASOL VGENRTMTIH NGMFFSTYDR

401 DNDGWVTTDP RKOCSKEDGG GWWYNRCHAA NPNGRYYWGG LYSWDMSKHG

451 TDDGVWWMNW KGSWYSMRRM SMKIRPFFPQ $Q$

\section{Show predicted peptides also}

\section{Sort Peptides By 0 Residue Number Increasing Mass Decreasing Mass}

$\begin{aligned} & \text { Start } \text { End } \\ & 43-62 \\ & 44-62 \\ & 154-170 \\ & 171-186 \\ & 202-214 \\ & 237-257 \\ & 264-275 \\ & 276-284 \\ & 305-318 \\ & 417-426 \\ & 427-435 \\ & 474-481\end{aligned}$

\section{Observed} 2105. 2007 1977.0981 2052.0364 1902.0759 1601.7582 2413. 3040 1374.7316 980.4769 1671.8080 1239. 5681

996.4788 1032. 5969

\section{Mr (expt) Mr (calc)} 2104.1934 2104.1065 $1976.0908 \quad 1976.0116$ $2051.0291 \quad 2050.9443$ $1901.0686 \quad 1900.9894$ $1600.7509 \quad 1600.6861$ 2412.2967 2412.1995 $1373.7243 \quad 1373.6688$ $\begin{array}{rr}979.4697 & 979.4359\end{array}$ $1670.8007 \quad 1670.7399$ $1238.5608 \quad 1238.5105$ $995.4715 \quad 995.4355$ $1031.5896 \quad 1031.5553$

ppm
41
40
41
42
40
40
40
34
36
41
36
33

\section{Miss Sequence}

1 R. KEEPPSLRPAPPPISGGGYR. A (Ions score 19)

0 K. EEPPSLRPAPPPISGGGYR.A (I ons score 17)

0 K. ENENVINEYSSILEDQR. L (IOnS score 51)

- R. LYIDETVNDNIPLNLR.V (IONS score 42)

0 K. LESDISAQMEYCR.T Carbamidomethyl (C) (Ions score 15)

1 R. KGGETSEMYLIQPDTSIKPYR.V (IOnS score 70)

- K. TENGGWTVIQNR.Q (IONS score 22)

- R. QDGSVDFGR.K (IOnS score 27)

0 K. YCGLPGEYWLGNDK. I Carbam1domethyl (C) (Ions score 15)

0 K. EDGGGWWYNR.C (IOns score 6)

- R. CHAANPNGR.Y Carbanidomethy 1 (C) (Ions score 33)

0 K. IRPFFPQQ. - (Ions score 18) 
Sample M19

4700 Reflector Spec \#1 MC=>NR(2.00)[BP = 1040.6, 44962]

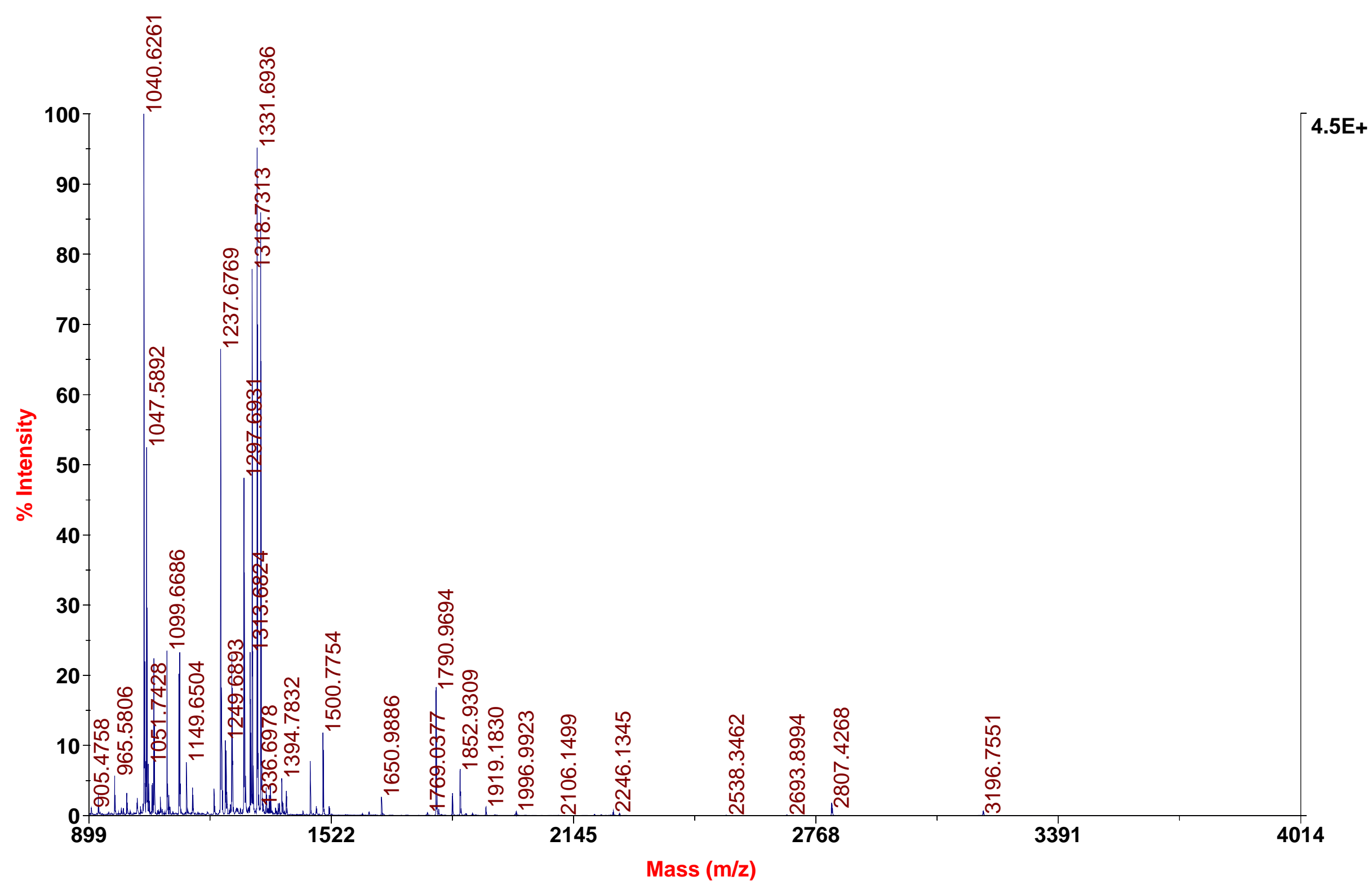




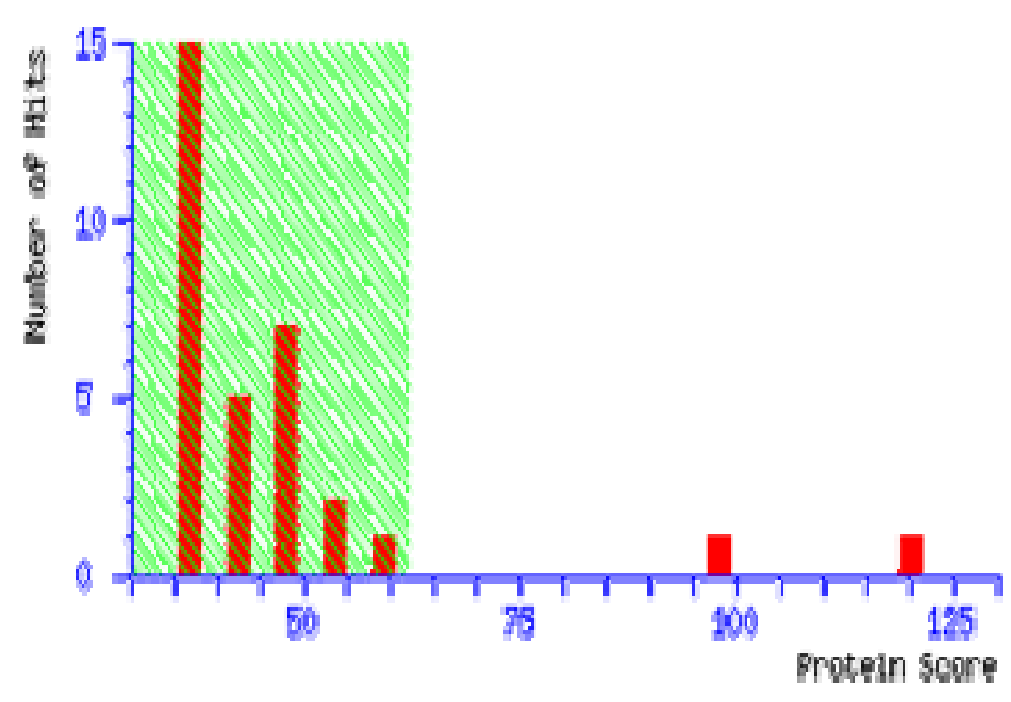

Concise Protein Summary Report

Format As Concise Protein Summary . Help

Significance threshold $p<0.05 \quad$ Max. number of hits AUTO

Re-Search All Search Unmatched

1. Q3V2G1 MOUSE Mass: 30666 Score: 120 Expect: 9.2e-08 Matches: 24

Adult male small intestine cDNA, RIKEN full-length enriched library, clone:2010012G12 product:apol1poprotein A-I, JC1237 Mass: 30358 Score: 113 Expect: 4.6e-07 Matches: 22

apolipoprote1n A-I precursor - mouse

Q8BPD5 MOUSE Mass: 30597 Score: 110 Expect: 9.2e-07 Matches: 23

18 days pregnant adult female placenta and extra embryonic t1ssue cDNA, RIKEN full-length enriched library, clone:

S22420 Mass: 30569 Score: 110 Expect: 9.2e-07 Matches: 22

apolipoprote1n A-I precursor - mouse 


\section{Protein View}

Match to: Q3V2G1_MOUSE Score: 120 Expect: 9.2e-08

Adult male small intestine cDNA, RIKEN full-length enriched library, clone:2010012G12 product:apolipoprotein Found 1n search of pmf_C6_129990866002.txt

Nom1nal mass $\left(\mathrm{M}_{\mathrm{x}}\right)$ : 30666; Calculated pI value: 5.64

NCBI BLAST search of Q3V2G1_MOUSE aga1nst nr

unformatted sequence string for pasting 1nto other applications

Taxonony: Mus musculus

Links to retrieve other entries conta1ning this sequence from NCBI Entrez:

(no taxonomy information for this entry)

Var1able modif1cations: Carbanidomethy1 (C), Dean1dated (NQ), ox1dation (M)

cleavage by Trypsin: cuts C-term side of $K R$ unless next residue $1 s \mathrm{~F}$

Number of mass values searched: 147

Number of mass values matched: 24

sequence Coverage: 608

Matched peptides shown 1n Bold Red

1 MKAVVLAVAL VFLTGRQAWH VWQQDEPQSQ WDKVKDFANV YVDAVKDSGR

51 DYVSQFESSS LGQQLNLNLL ENWDTLGSTV SQLQERLGPL TRDFWDNLEK

101 ETDWVRQEMN KDLEEVKQKV QPYLDEFQKK WKEDVELYRO KVAPLGAELQ

151 ESARQKLQEL QGRLSPVAEE FRDRMRTHVD SLRTQLAPHS EQMRESLAQR

201 LAELKSNPTL NEYHTRAKTH LKTLGEKARP ALEDLRHSLM PMLETLKTQV

251 QSVIDKASET LTAQ

Show predicted peptides also

\section{Sort Peptides By Residue Number Increasing Mass Decreasing Mass}

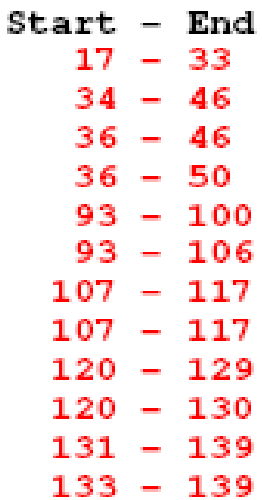

ppm
72
38
41
28
32
42
49
49
37
36
43
49

\section{Miss Sequence}

0 R. QAWHVWQQDEPQSQWDK.V 2 Deam1dated (NQ)

1 K. VKDEANVYVDAVK. D

0 K. DFANVYVDAVK. D

1 K. DFANVYVDAVKDSGR.D

O R. DFWDNLEK. E

1 R. DFWDNLEKETDWVR. $Q$

1 R. QEMNKDLEEVK. $Q$

1 R. QEMNKDLEEVK. $Q$

O K. VQPYLDEFQK. K

1 K. VQPYLDEFQKK. W

1 K. WKEDVELYR.Q

$\begin{array}{ll}0 & \text { K. EDVELYR. Q }\end{array}$ 


\section{M19}

4700 MS/MS Precursor 1040.63 Spec \#1 MC[BP = 884.4, 8838]

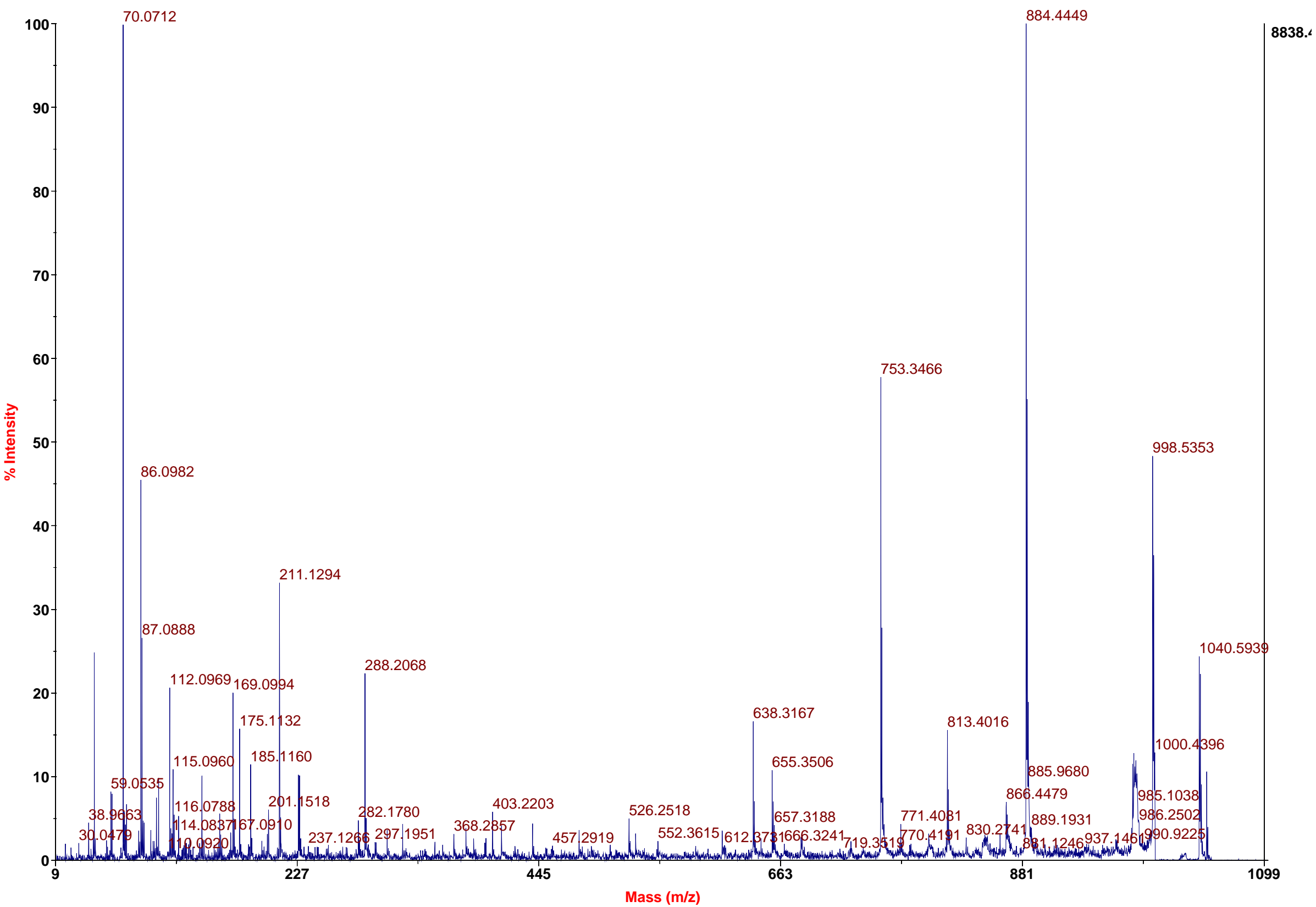




\section{M19}

4700 MS/MS Precursor 1340.78 Spec \#1 MC[BP $=70.1,10449]$






\section{Protein View}

Match to: Q3V2G1_MOUSE score: 268

Adult male small intestine cDNA, RIKEN full-length enriched library, clone:2010012G12 product:apolipoprotein A-I, Found 1n search of ppw_C6_129990871602.txt

Nom1nal mass $\left(M_{x}\right)$ : 30666; Calculated pI value: 5.64

NCBI BLAST search of Q3V2G1_MOUSE against $\mathrm{nr}$

Unformatted sequence string for pasting 1nto other applications

Taxonony: Mus musculus

Links to retrieve other entries containing this sequence from NCBI Entrez:

(no taxonomy information for this entry)

Var1able mod1fications: Carbanidomethyl (C), Dean1dated (NQ), ox1dation (M)

cleavage by Trypsin: cuts C-term side of $K R$ unless next residue is $P$

sequence coverage: 428

Matched peptides shown 1n Bold Red

1 MKAVVLAVAL VFLTGRQAWH VWQQDEPQSQ WDKVKDFANV YVDAVKDSGR

51 DYVSQFESSS LGQQLNLNLL ENWDTLGSTV SQLQERLGPL TRDFWDNLEK

101 ETDWVRQEMN KDLEEVKQKV QPYLDEFQKK WKEDVELYRQ KVAPLGAELQ

151 ESARQKLQEL QGRLSPVAEE FRDRMRTHVD SLRTQLAPHS EQMRESLAQR

201 LAELKSNPTL NEYHTRAKTH LKTLGEKARP ALEDLRHSLM PMLETLKTQV

251 QSVIDKASET LTAQ

Show predicted peptides also

\section{Sort Peptides By $\odot$ Residue Number Increasing Mass $\bigcirc$ Decreasing Mass}

$\begin{array}{rrrr}\text { Start - End } & \text { Observed } & \text { Mr (expt) } & \text { Mr(calc) } \\ 34-46 & 1467.8397 & 1466.8324 & 1466.7769 \\ 93-106 & 1852.9277 & 1851.9204 & 1851.8428 \\ 120-129 & 1266.6832 & 1265.6759 & 1265.6292 \\ 120-130 & 1394.7819 & 1393.7746 & 1393.7242 \\ 131-139 & 1237.6746 & 1236.6673 & 1236.6139 \\ 142-154 & 1340.7750 & 1339.7677 & 1339.7095 \\ 155-163 & 1099.6685 & 1098.6612 & 1098.6145 \\ 164-172 & 1047.5887 & 1046.5814 & 1046.5396 \\ 164-174 & 1318.7311 & 1317.7238 & 1317.6677 \\ 184-194 & 1297.6907 & 1296.6834 & 1296.6245 \\ 206-216 & 1331.6930 & 1330.6857 & 1330.6266 \\ 228-236 & 1040.6257 & 1039.6184 & 1039.5774\end{array}$

\begin{tabular}{|c|c|c|}
\hline pm & iss & Sequence \\
\hline 38 & 1 & K. VKDFANVYVDAVK. D \\
\hline 42 & 1 & R. DFWDNLEKETDWVR. Q \\
\hline 37 & 0 & K. VQPYLDEFQK.K (IOnS score 40) \\
\hline 36 & 1 & K. VQPYLDEFQKK. W (Ions score 38 ) \\
\hline 43 & 1 & K. WKEDVELYR.Q (IONS score 64) \\
\hline 43 & 0 & K. VAP LGAELQESAR. $\bar{Q}$ \\
\hline 43 & 1 & (Ions score 39) \\
\hline 40 & 0 & (Ions score 31) \\
\hline 43 & 1 & R. LSPVAEEFRDR. M \\
\hline 45 & 0 & R. TQLAPHSEQMR. E \\
\hline 44 & 0 & K. SNPTLNEYHTR. A \\
\hline 39 & 0 & (Ions score 17) \\
\hline
\end{tabular}


Sample M20

4700 Reflector Spec \#1 MC=>NR(2.00)[BP = 906.5, 19962]

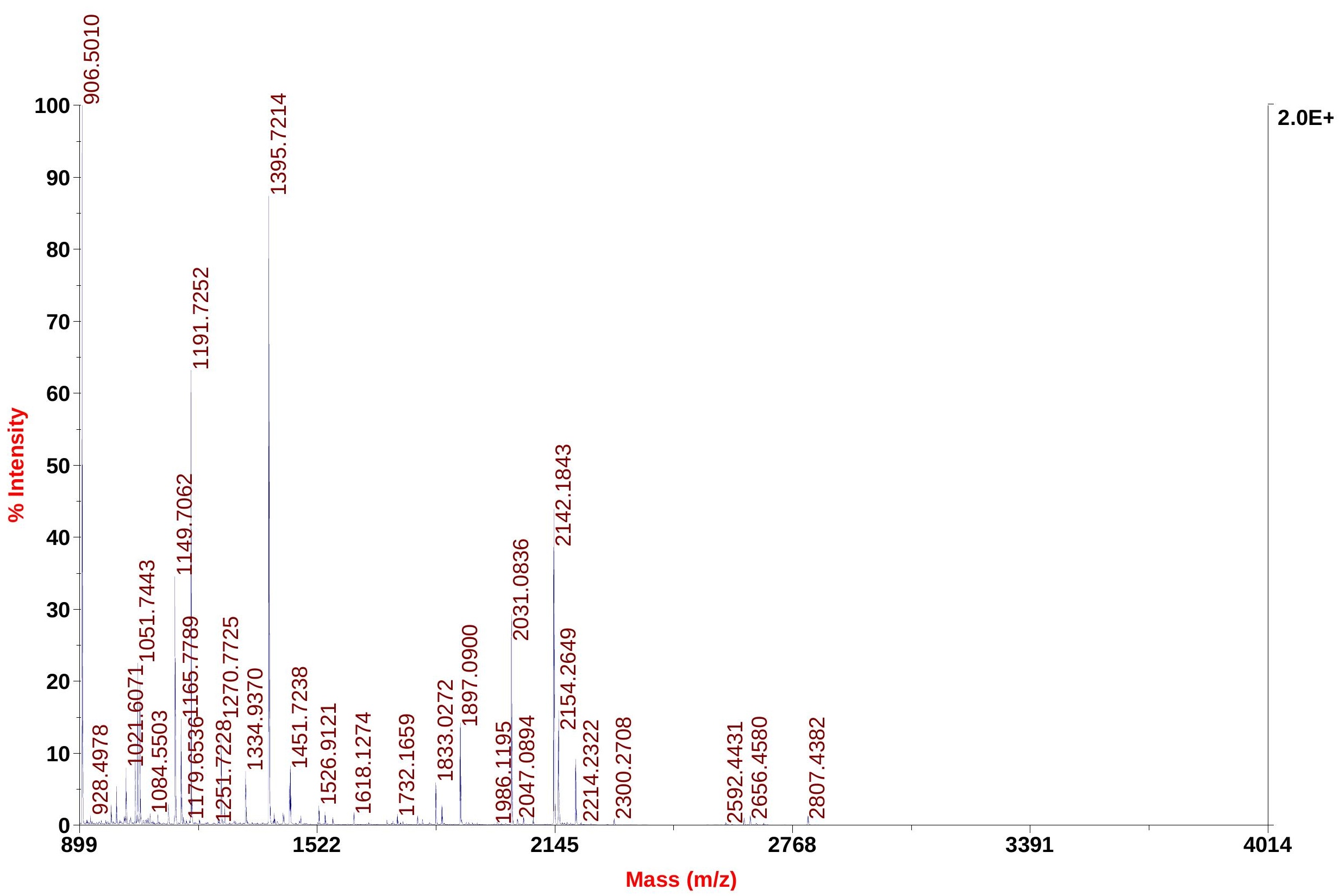




\section{Protein View}

Match to: PRDX6_MOUSE Score: 176 Expect: 2.3e-13 Peroxiredoxin-6 (EC 1.11.1.15) (Antioxidant protein 2) (1-Cys peroxiredoxin) (1-Cys PRX) (Acidic calci Found 1n search of pmf_D6_129990866103.txt

Nomlnal mass $\left(M_{x}\right): 24724$; calculated pI value: 5.72 NCBI BLAST search of PRDX6 MOUSE aga1nst nr unformatted sequence string for pasting 1nto other applications

Taxonony: Mus musculus

Var1able modif1cat1ons: Carban1domethyl (C), Dean1dated (NQ), ox1dation (M) cleavage by Trypsin: cuts c-term side of KR unless next residue $1 s$ P Number of mass values searched: 119

Number of mass values matched: 30 sequence Coverage: $88 \%$

Matched peptides shown 1n Bold Red

1 PGGLLLGDEA PNFEANTTIG RIRFHDELGD SWGILFSHPR DFTPVCTTEI

51 GRAAKLAPEF AKRNVKLIAL SIDSVEDHLA WSKDINAYNG ETPTEKLPFP 101 IIDDKGRDLA ILLGMLDPVE KDDNNMPVTA RVVFIFGPDK KLKLSILYPA

151 TTGRNFDEIL RVVDSLQLTG TKPVATPVDW KKGESVMVVP TLSEEEAKQC

201 FPKGVFTKEL PSGKKYLRYT PQP

Show predicted peptides also

\section{Sort Peptides By}

$\begin{aligned} \text { Start } & - \text { End } \\ 1 & -21 \\ 22 & -40 \\ 24 & -40 \\ 41 & -52 \\ 41 & -52 \\ 53 & -62 \\ 64 & -83 \\ 67 & -83 \\ 84 & -96 \\ 97 & -105 \\ 97 & -107 \\ 106 & -121 \\ 108 & -121 \\ 108 & -121\end{aligned}$

- Residue Number

Observed
2142.1814
2300.2776
2031.0808
1338.7070
1395.7209
1045.6050
2239.2366
1897.0906
1451.7247
1057.6310
1270.7704
1740.0472
1526.9110
1542.9082

\section{Mr (expt)} 2141. 1741 2299.2703 2030.0735 1337.6997 1394. 7136 1044.5977 2238.2293 1896.0833 1450.7174 1056.6237 1269.7631 1739.0399 1525.9037 1541.9009
Increasing Mass

Decreasing Mass

$M r$ (calc)
2141.0753
2299.1651
2029.9799
1337.6286
1394.6500
1044.5967
2238.1896
1895.9993
1450.6576
1056.5855
1269.7081
1738.9651
1525.8425
1541.8375

$\mathrm{Mr}$ (calc)

2299.1651

2029.9799

1044.5967

1450.6576

1269.7081

1541,8375

ppm
46
46
46
53
46
1
18
44
41
36
43
43
40
41

\section{Miss Sequence}

- . PGGLLLGDEAPNEEANTTIGR . I

R. IRFHDF LGDSWGILFSHPR. D

R. FHDFLGDSWGILESHPR. D

R. DFTPVCTTELGR . A

R. DFTPVCTTELGR.A Carban1 domethy 1

R. AAKLAPEFAK . $R$

R. NVKLIALSIDSVEDHLAWSK. D Dean1dated

K. LIALSIDSVEDHLAWSK. D

K. DINAYNGETPTEK . L

K. LPFPI IDDK, G

K. LPFPI IDDKGR. D

K. GRDLA I LLGMLDPVEK. D

R. DLA ILLGMLDPVEK. D

R. DLAILLGMLDPVEK. D ox1dat1on 


\section{M20}

4700 MSIMS Precursor 906.502 Spec \#1 MC[BP = 175.1, 3389]

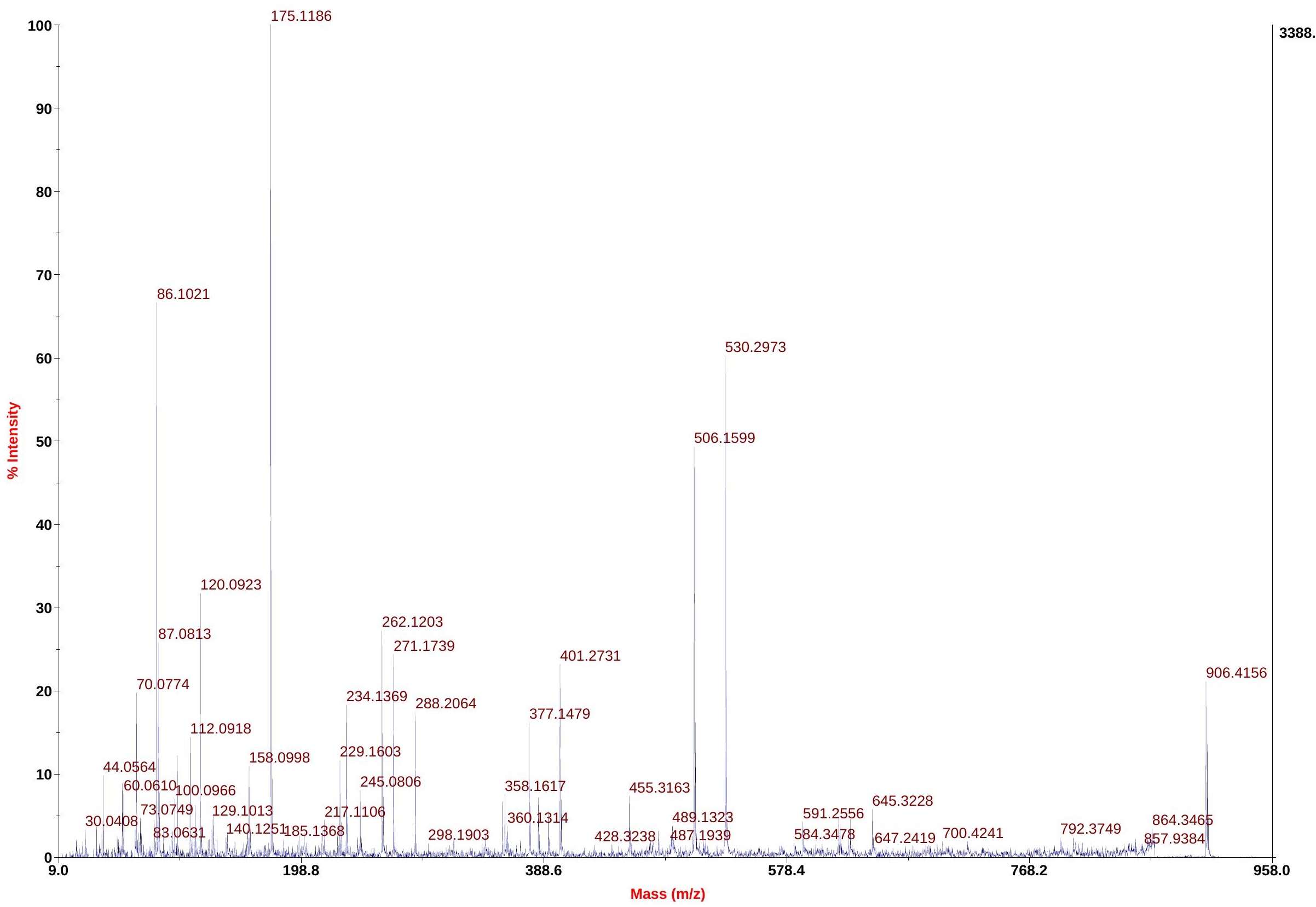




\section{M20}

4700 MS/MS Precursor 1149.7 Spec \#1 MC[BP = 544.3, 2164]

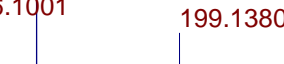

346.2023

341.1654

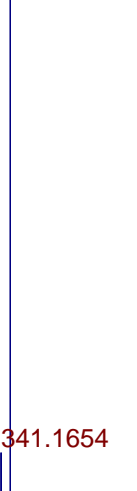

202.1070

233.1650

127.0878

123.1121

261.1431

398.1849

$\begin{array}{cc}398.1849 & \\ 38546 & 459.2655\end{array}$

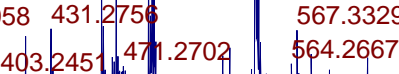

250.2 


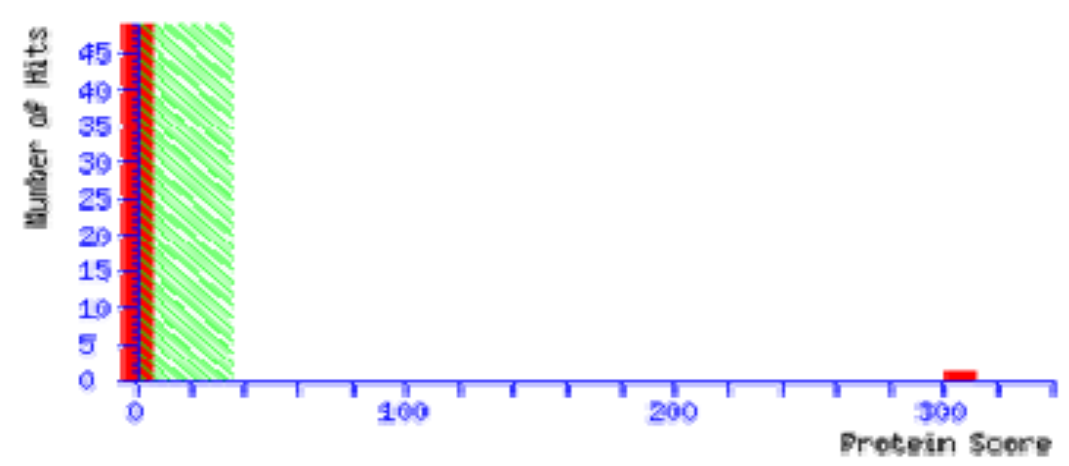

\section{Peptide Summary Report}

\section{Format As Peptide Summary}

Significance threshold $\mathrm{p}<0.05$

Standard scoring

Show pop-ups Suppress pop-ups
Max. number of hits AUTO

Ions score or expect cut-off 0

Sort unassigned Decreasing Score $\underline{\text { Help }}$

Show sub-sets 0

Require bold red

\section{Error tolerant}

PRDX6_MOUSE Mass: 24724 Score: 306 Matches: 13 (11) Sequences: $13(11)$ emPAI: 17.46

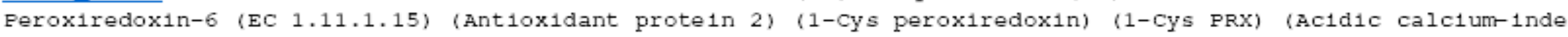
$\square$ check to 1 nclude this hit in error tolerant search

\begin{tabular}{|c|}
\hline 同 \\
\hline$\nabla$ \\
\hline$\nabla$ \\
\hline 四 \\
\hline 四 \\
\hline$\square$ \\
\hline$\nabla$ \\
\hline 回 \\
\hline $\begin{array}{l}\text { 口 } \\
\end{array}$ \\
\hline $\begin{array}{l}\nabla \\
\square\end{array}$ \\
\hline $\begin{array}{l}\nabla \\
\nabla\end{array}$ \\
\hline 目 \\
\hline
\end{tabular}

$\begin{array}{rrr}\text { Observed } & \text { Mr(expt) } & \text { Mr(calc) } \\ 906.5024 & 905.4952 & 905.4607 \\ 1021.6072 & 1020.5999 & 1020.5644 \\ 1057.6310 & 1056.6237 & 1056.5855 \\ 1149.7048 & 1148.6975 & 1148.6594 \\ 1191.7252 & 1190.7179 & 1190.6659 \\ 1270.7704 & 1269.7631 & 1269.7081 \\ 1395.7209 & 1394.7136 & 1394.6500 \\ 1451.7247 & 1450.7174 & 1450.6576 \\ 1833.0288 & 1832.0215 & 1831.9237 \\ 1897.0906 & 1896.0833 & 1895.9993 \\ 2031.0808 & 2030.0735 & 2029.9799 \\ 2142.1814 & 2141.1741 & 2141.0753 \\ 2154.2661 & 2153.2588 & 2153.1732\end{array}$

$\begin{array}{lr}\text { ppm } & \text { Mis } \\ 38.1 & 0 \\ 34.8 & 0 \\ 36.1 & 0 \\ 33.2 & 1 \\ 43.7 & 0 \\ 43.3 & 1 \\ 45.6 & 0 \\ 41.3 & 0 \\ 53.4 & 1 \\ 44.3 & 0 \\ 46.1 & 0 \\ 46.2 & 0 \\ 39.7 & 0\end{array}$

Score
40
43
59
35
36
42
71
45
24
72
30
71
55

Expect Rank Unique

0.0311

0.00881

0.000241

0.0441

0.0341

0.00981

2. $3 e-05 \quad 1$

0.00951

0.941

1. $2 e-05$

0.281

2. $2 e-05 \quad 1$

$0.00047 \quad 1$

\section{Peptide}

R. NFDEILR.V

R. VVFIFGPDK . K

K. LPFPI IDDK . $G$

R. VVFIFGPDKK . L

K. LSILYPATTGR. N

K. LPFPI IDDKGR. D

R. DFTPVCTTELGR.A + Carbamidometl

K. DINAYNGETPTEK . L

K. KGESVMVVPTLSEEEAK. $Q$

K. LIALSIDSVEDHLAWSK. D

R. FHDFLGDSWGILESHPR. D

-. PGGLLLGDEAPNEEANTTIGR I I

R. VVDSLQLTGTKPVATPVDWK. K 


\section{Protein View}

Match to: PRDX6_MOUSE Score: 306

Peroxiredoxin-6 (EC 1.11.1.15) (Antioxidant protein 2) (1-Cys peroxiredoxin) (1-Cys PRX) (Acidic calcium-independent Found 1n search of Ppw_D6_129990871703.txt

Nom1nal mass $\left(M_{x}\right): 24724$; Calculated pI value: 5.72

NCBI BLAST search of PRDX6_MOUSE aga1nst nr

unformatted sequence string for pasting into other applications

Taxonony: Mus musculus

Var1able modifications: Carbanidomethyl (C), Deanldated (NQ), ox1dation (M)

cleavage by Trypsin: cuts c-term. side of $\mathrm{KR}$ unless next residue $1 \mathrm{~s}$

sequence Coverage: 698

Matched peptides shown in Bold Red

1 PGGLLLGDEA PNFEANTTIG RIRFHDFLGD SWGILFSHPR DFTPVCTTEL

51 GRAAKLAPEF AKRNVKLIAL SIDSVEDHLA WSKDINAYNG ETPTEKLPFP

101 IIDDKGRDLA ILLGMLDPVE KDDNNMPVTA RVVFIFGPDK KLKLSILYPA

151 TTGRNFDEIL RVVDSLQLTG TKPVATPVDW KKGESVMVVP TLSEEEAKQC

201 FPKGVFTKEL PSGKKYLRYT PQP

Show predicted peptides also

Sort Peptides By

$\begin{array}{rrrr}\text { Start - End } & \text { Observed } & \text { Mr(expt) } & \text { Mr(calc) } \\ 1-21 & 2142.1814 & 2141.1741 & 2141.0753 \\ 24-40 & 2031.0808 & 2030.0735 & 2029.9799 \\ 41-52 & 1395.7209 & 1394.7136 & 1394.6500 \\ 67-83 & 1897.0906 & 1896.0833 & 1895.9993 \\ 84-96 & 1451.7247 & 1450.7174 & 1450.6576 \\ 97-105 & 1057.6310 & 1056.6237 & 1056.5855 \\ 97-107 & 1270.7704 & 1269.7631 & 1269.7081 \\ 132-140 & 1021.6072 & 1020.5999 & 1020.5644 \\ 132-141 & 1149.7048 & 1148.6975 & 1148.6594 \\ 144-154 & 1191.7252 & 1190.7179 & 1190.6659 \\ 155-161 & 906.5024 & 905.4952 & 905.4607 \\ 162-181 & 2154.2661 & 2153.2588 & 2153.1732 \\ 182-198 & 1833.0288 & 1832.0215 & 1831.9237\end{array}$

Decreasing Mass

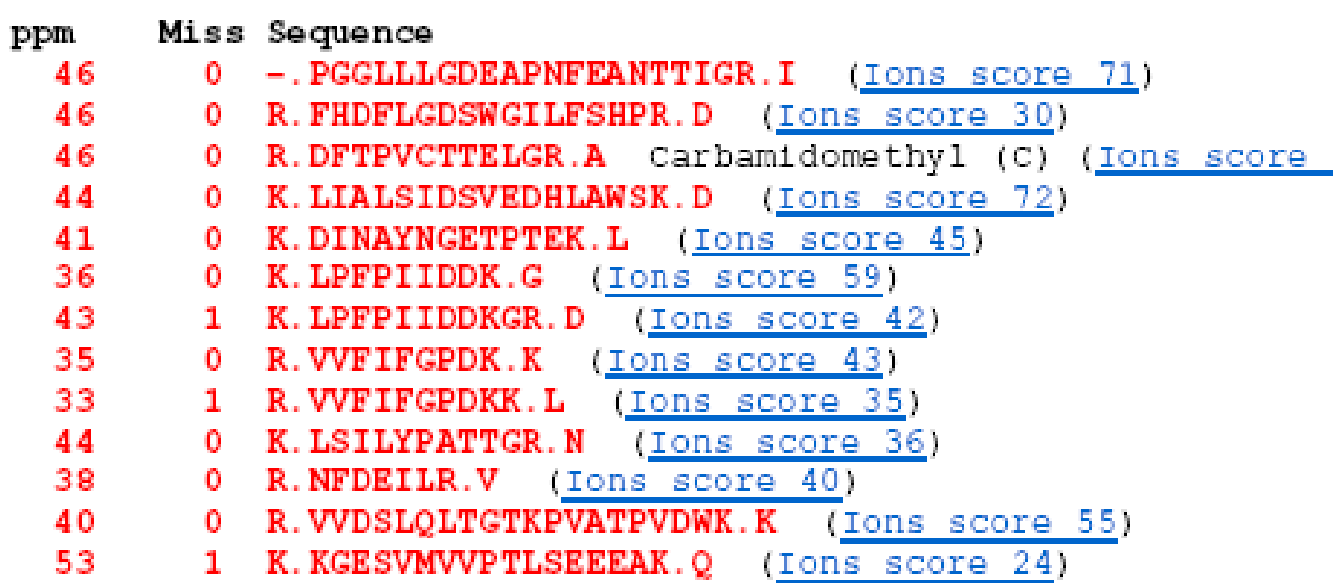


Sample M21

4700 Reflector Spec \#1 MC=>NR(2.00)[BP = 1149.6, 49685]






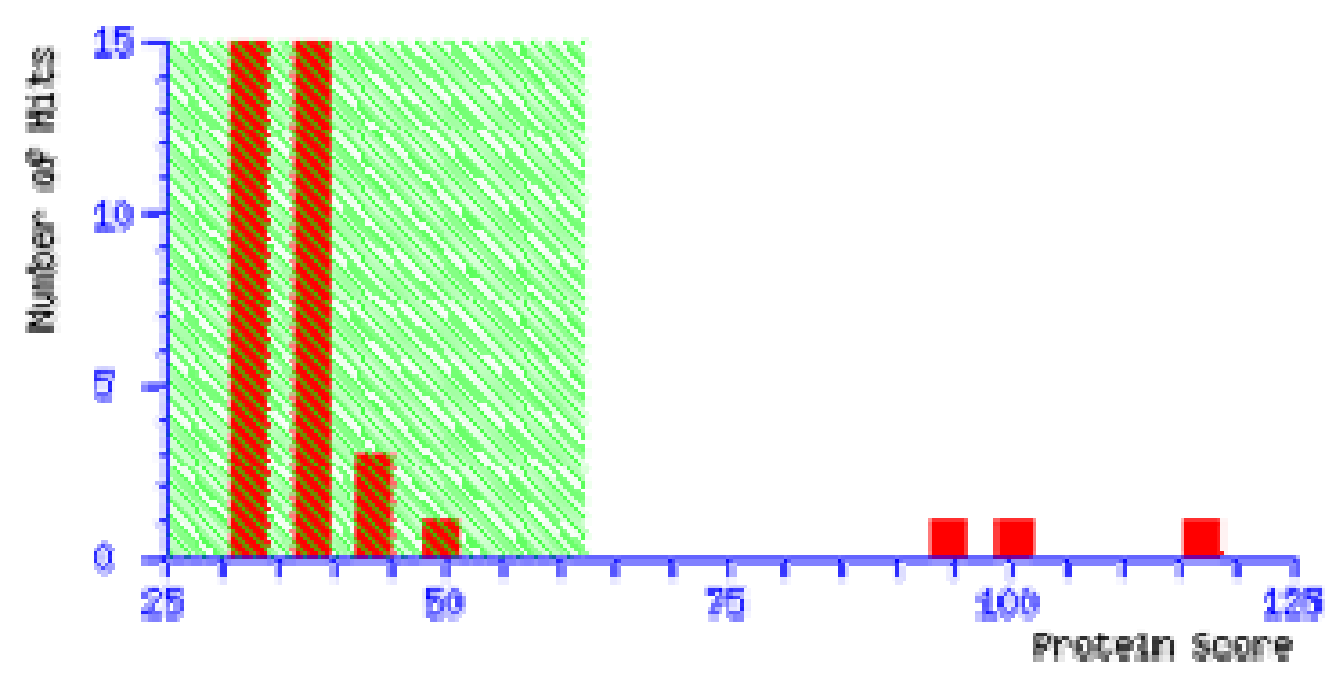

\section{Concise Protein Summary Report}

Format As Concise Protein Summary

Significance threshold $p<0.05$

Re-Search All

Search Unmatched

1.

JN0679 Mass: 23000 Score: 117
heat shock prote1n 25 - nouse
S02143 Mass: 22868 Score: 106
stress prote1n, $25 \mathrm{~K}-$ mouse
CAB37341 Mass: 22838 Score: 94
MURSPH NID: - Mus sp.
A53423
HSP27-related prote1n (form a) - mouse

$\underline{\text { Help }}$

Max. number of hits AUTO

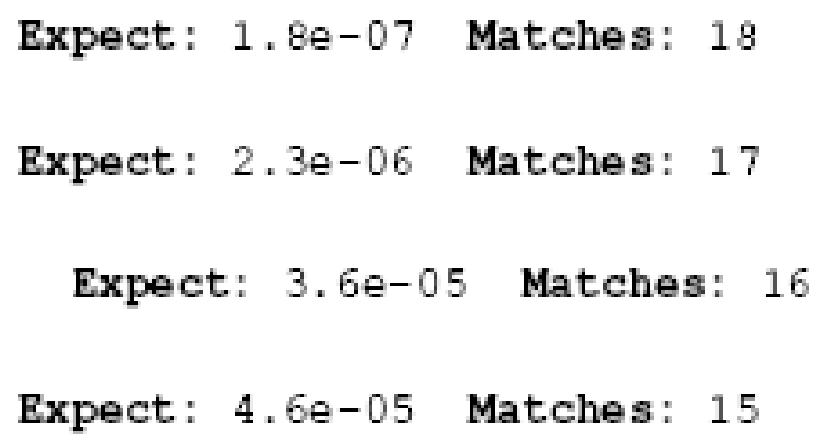




\section{Protein View}

Match to: JNo679 score: 117 Expect: 1.8e-07

heat shock protein 25 - mouse

Found $1 \mathrm{n}$ search of pmf_E6_129990866104.txt

Nomlnal mass $\left(M_{x}\right)$ : 23000; calculated pI value: 6.12

NCBI BLAST search of JN0679 aga1nst nr

unformatted sequence string for pasting 1nto other applications

Taxonony: Mus musculus

L1nks to retrieve other entries contalning this sequence from NCBI Entrez:

(no taxonomy information for this entry)

(no taxonomy information for this entry)

(no taxonomy 1 nformation for this entry)

(no taxonomy information for this entry)

(no taxonomy information for this entry)

(no taxonomy 1 nformation for this entry)

(no taxonomy information for this entry)

(no taxonomy information for this entry)

(no taxonomy information for this entry)

Var1able mod1f1cat1ons: Carban1donethyl (C), Dean1dated (NQ), ox1dat1on cleavage by Trypsin: cuts c-term side of KR unless next residue $1 s$ P Number of mass values searched: 110 Number of mass values matched: 18

sequence coverage: $71 \%$

Matched peptides shown in Bold Red
1 MTERRVPFSL
51 GWPGYVRELP
101 VSLDVNHFAP
LRSPSWEPER DWYPAHSRLE
DQAFGVPRLP DEWSOWFSAA
LNROLSSGVS EIROTADRWR
151 GVDPTLVSSS
EELTVKTKEG VVEITGKHEE RODEHGYISR CFTRKYTLPP
201 GKSEQSGAK

Show predicted peptides also

\section{Sort Peptides By}

Start - End
$5-12$
$6-20$
$13-20$
$13-28$
$21-28$
$29-38$
$84-93$
$101-116$
$117-127$
$119-127$

Residue Number

\section{Obse rved}

987.6417

1818.0321

1005.5128

2018.0139

1031.5046

1149.6465

1075.6113

1798.0094

1160.6812

931.5301

\section{Mr (expt)}

986.6344

1817.0248

1004.5055

2017.0066

1030.4973

1148.6392

1074.6040

1797.0021

1159.6739

930.5228
Increasing Mass

Mr (calc)
986.6025
1816.9624
1004.4716
2016.9231
1030.4621
1148.5979
1074.5669
1796.9309
1159.6449
930.5022

986.6025

16.9624

1030.4621

1159.6449

930.5022
Decreasing Mass

ppm
32
34
34
41
34
36
35
40
25
22

(M) 


\section{M21}

4700 MS/MS Precursor 1149.65 Spec \#1 MC[BP = 774.4, 6510]

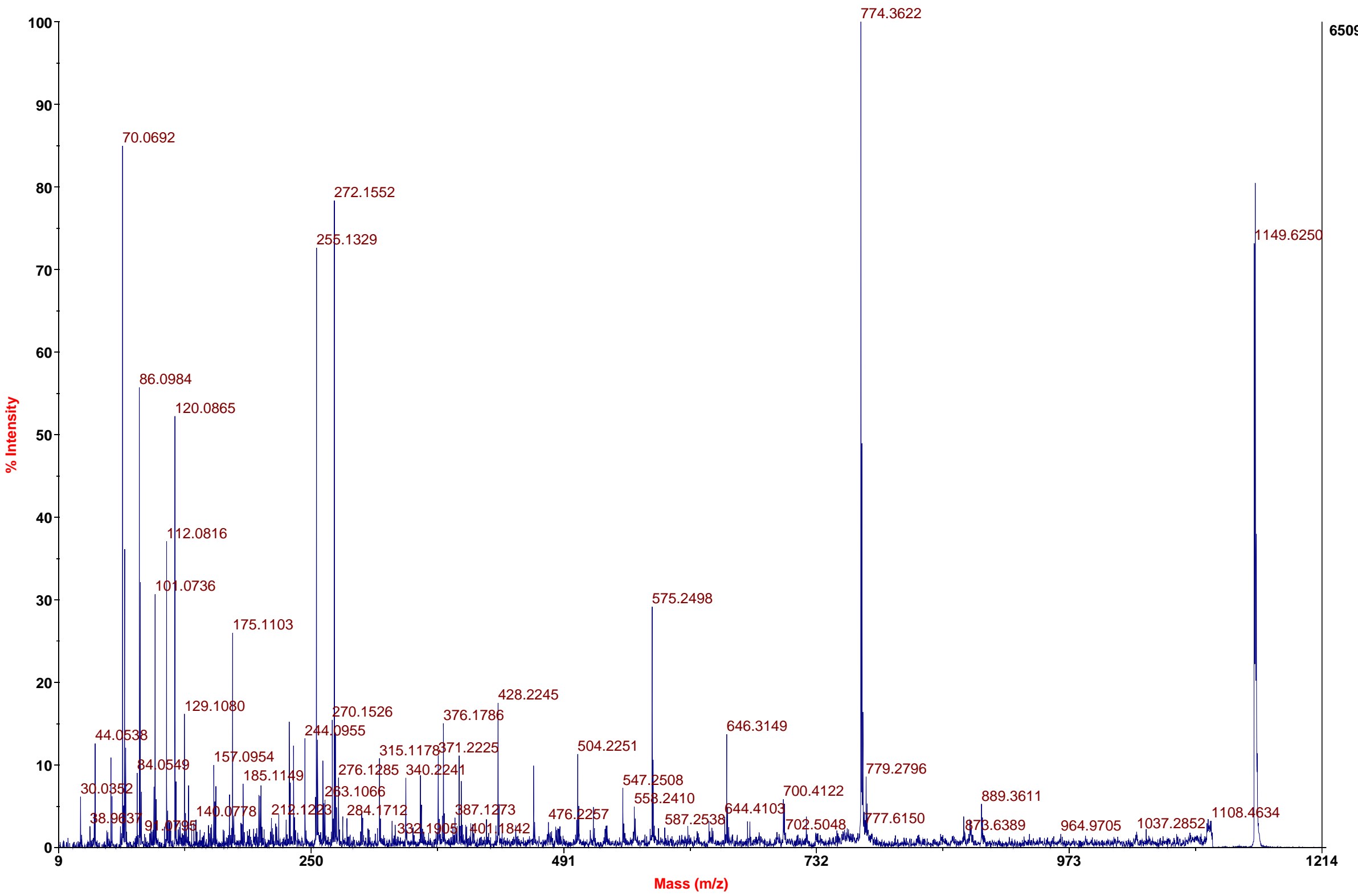




\section{M21}

4700 MS/MS Precursor 1051.74 Spec \#1 MC[BP = 102.1, 2464]








\section{Peptide Summary Report}

Format As Peptide Summary

Significance threshold $p<0.05$

Standard scoring MudPIT scoring Ions score or expect cut-off 0

Show pop-ups Suppress pop-ups $\bigcirc$ Sort unassigned Decreasing Score $\underline{\text { Help }}$

Show sub-sets 0

Require bold red

\section{Select All Select None Search Selected $\square$ Error tolerant}

1. JN0679 Mass: 23000 Score: 160 Matches: 9(5) Sequences: 9 (5) emPAI: 2.46

heat shock prote1n 25 - nouse

$\square$ check to include this hit in error tolerant search

\begin{tabular}{|c|c|c|c|c|c|c|c|c|c|c|c|}
\hline \multicolumn{2}{|c|}{ Query } & Observed & $\operatorname{Mr}$ (expt) & $\operatorname{Mr}(\mathrm{calc})$ & ppm & Miss & Score & Expect & Rank & Unique & Peptide \\
\hline 目 & $\underline{1}$ & 927.5178 & 926.5105 & 926.4821 & 30.7 & 0 & 36 & 0.051 & 1 & U & R. AQIGGPEAGK.S \\
\hline 目 & $\underline{2}$ & 987.6417 & 986.6344 & 986.6025 & 32.4 & 1 & 18 & 2.1 & 1 & $\mathrm{U}$ & R. RVPFSLLR. S \\
\hline D & $\underline{6}$ & 1031.5046 & 1030.4973 & 1030.4621 & 34.2 & 0 & 22 & 2.3 & 1 & $\mathrm{U}$ & R. DWYPAHSR. L \\
\hline 目 & $\underline{9}$ & 1075.6113 & 1074.6040 & 1074.5669 & 34.5 & 0 & 46 & 0.0067 & 1 & $\mathrm{u}$ & R. QLSSGVSEIR. $Q$ \\
\hline 目 & $\underline{12}$ & 1149.6465 & 1148.6392 & 1148.5979 & 36.0 & 0 & 62 & 0.00016 & 1 & $\mathrm{U}$ & R. LFDQAFGVPR . L \\
\hline D & 14 & 1798.0094 & 1797.0021 & 1796.9309 & 39.7 & 0 & 68 & 3. $6 e-05$ & 1 & $\mathrm{U}$ & R. VSLDVNHFAPEELTVK. T \\
\hline
\end{tabular}




\section{Protein View}

Match to: JN0679 Score: 160

heat shock protein 25 - mouse

Found in search of ppw_E6_129990871804.txt

Nominal mass $\left(M_{r}\right): 23000$; Calculated pI value: 6.12

NCBI BLAST search of JNO679 against $\mathrm{nr}$

Unformatted sequence string for pasting into other applications

Taxonomy: Mus musculus

Links to retrieve other entries containing this sequence from NCBI Entrez:

(no taxonomy information for this entry)

(no taxonomy information for this entry)

(no taxonomy information for this entry)

(no taxonomy information for this entry)

(no taxonomy information for this entry)

(no taxonomy information for this entry)

(no taxonomy information for this entry)

(no taxonomy information for this entry)

(no taxonomy information for this entry)

Variable modifications: Carbamidomethyl (C), Deamidated (NQ), Oxidation (M)

cleavage by Trypsin: cuts C-term side of KR unless next residue is P

Sequence Coverage: 568

Matched peptides shown in Bold Red

1 MTERRVPFSL LRSPSWEPFR DWYPAHSRLF DQAFGVPRLP DEWSQWFSAA

51 GWPGYVRPLP AATAEGPAAV TLAAPAFSRA LNRQLSSGVS EIRQTADRWR

101 VSLDVNHFAP EELTVKTKEG VVEITGKHEE RQDEHGYISR CFTRKYTLPP

151 GVDPTLVSSS LSPEGTLTVE APLPKAVTQS AEITIPVTFE ARAQIGGPEA

201 GKSEQSGAK

Show predicted peptides also

Sort Peptides By $\odot$ Residue Number Increasing Mass Decreasing Mass

$\begin{aligned} \text { Start } & - \text { End } \\ 5 & -12 \\ 13 & -28 \\ 21 & -28 \\ 29 & -38 \\ 84 & -93 \\ 101 & -116 \\ 145 & -175 \\ 176 & -192 \\ 193 & -202\end{aligned}$

Observed

987.6417

2018.0139

1031.5046

1149.6465

1075.6113

1798.0094

3193.8574

1833.0530

927.5178

\section{Mr (expt) Mr (calc)}

986.6344 2017.0066 1030.4973 1148.6392 1074.6040 1797.0021 3192.8501 1832.0457 926.5105

\subsection{5}

2016.9231

1030.4621

1148.5979

1074.5669

1796.9309

3192.7170

1831.9680

926.4821 ppm

32

41

34

36

35

40

42

42

31

\section{Miss Sequence}

1 R.RVPFSLLR.S (Ions score 18)

1 R. SPSWEPFRDWYPAHSR. L (Ions score 14)

o R.DWYPAHSR.L (Ions score 22)

o R. LFDQAFGVPR. L (Ions score 62)

o R.QLSSGVSEIR. $Q$ (Ions score 46)

o R.VSLDVNHFAPEELTVK. T (Ions score 68)

1 R. KYTLPPGVDPTLVSSSLSPEGTLTVEAPLPK . A

O K.AVTQSAEITIPVTFEAR.A (Ions score 69)

o R.AQIGGPEAGK.S (Ions score 36) 


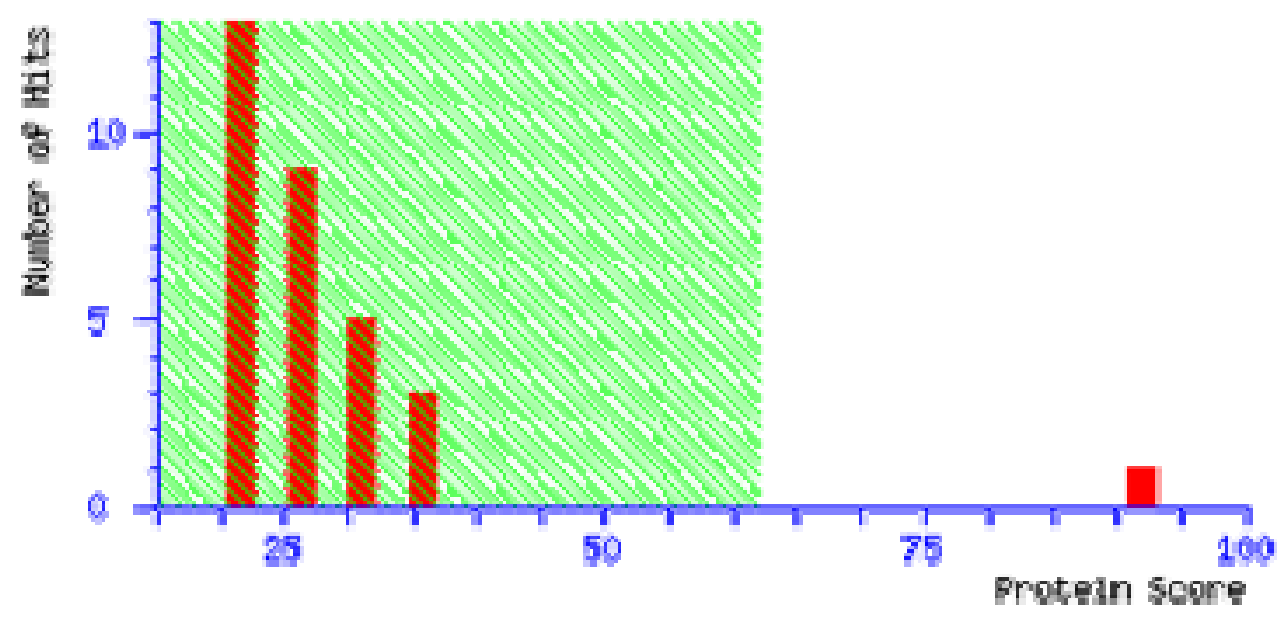

\section{Concise Protein Summary Report}

\section{Format As Concise Protein Summary .}

Significance threshold $p<0.05 \quad$ Max. number of hits AUTO

\section{Re-Search All Search Unmatched}

1. GDIS MOUSE Mass: 22705 Score: 92 Expect: 6.1 e-05 Matches: 17

Rho GDP-d1ssociation 1nh1b1tor 2 (Rho GDI 2) (Rho-GDI beta) (D4).- Mus musculus (Mouse I49687 Mass: 22836 Score: 92 Expect: $6.2 e-05$ Matches: 17

GDP-d1ssoc1ation inhibitor - mouse 


\section{Protein View}

Match to: GDIS_MOUSE Score: 92 Expect: 6.1e-05

Rho GDP-dissociation inhibitor 2 (Rho GDI 2) (Rho-GDI beta) (D4).- Mus musculus (Mouse).

Found 1n search of pmf_F6_129990866105.txt

Nom1nal mass $\left(M_{x}\right)$ : 22705; Calculated pI value: 4.97

NCBI BLAST search of GDIS_MOUSE aga1nst nr

unformatted sequence string for pasting 1nto other applications

Taxonony: Mus musculus

Var1able mod1f1cat1ons: Carban1domethyl (C), Dean1dated (NQ), ox1dation (M)

cleavage by Trypsin: cuts c-term side of KR unless next residue $1 \mathrm{~s} F$

Number of mass values searched: 97

Number of mass values matched: 17

sequence coverage: 818

Matched peptides shown 1n Bold Red

1 TEKDAQPQLE EADDDLDSKL NYKPPPQKSL KELOEMDKDD ESLTKYKKTL

51 LGDVPVVADP TVPNVTVTRL SLVCDSAPGP ITMDLTGDLE ALKKDTFVLK

101 EGIEYRVKIN FKVNKDIVSG LKYVQHTYRT GMRVDKATFM VGSYGPRPEE

151 YEFLTPVEEA PKGMLARGTY HNKSFFTDDD KQDHLTWEWN LAIKRDWTE

Show predicted peptides also

\section{Sort Peptides By}

$\begin{aligned} \text { Start } & - \text { End } \\ 4 & -19 \\ 4 & -28 \\ 20 & -28 \\ 32 & -45 \\ 32 & -45 \\ 48 & -69 \\ 49 & -69 \\ 70 & -93 \\ 70 & -94 \\ 70 & -94 \\ 95 & -106 \\ 113 & -122 \\ 123 & -129 \\ 137 & -162 \\ 137 & -162 \\ 174 & -194 \\ 182 & -194\end{aligned}$

- Residue Number

Increasing Mass
Decreasing Mass

Observed
1788.8712
2854.5359
1084.6661
1680.8522
1696.8540
2291.4194
2163.3232
2516.4006
2644.5061
2660.5107
1469.8688
1072.6866
966.5283
2944.5640
2960.5784
2609.3772
1653.9197

$\begin{array}{rr}\text { Mr (expt) } & \text { Mr(calc) } \\ 1787.8639 & 1787.7697 \\ 2853.5286 & 2853.3668 \\ 1083.6588 & 1083.6077 \\ 1679.8449 & 1679.7560 \\ 1695.8467 & 1695.7509 \\ 2290.4121 & 2290.2897 \\ 2162.3159 & 2162.1947 \\ 2515.3933 & 2515.2550 \\ 2643.4988 & 2643.3499 \\ 2659.5034 & 2659.3448 \\ 1468.8615 & 1468.7562 \\ 1071.6793 & 1071.6288 \\ 965.5210 & 965.4719 \\ 2943.5567 & 2943.4000 \\ 2959.5711 & 2959.3950 \\ 2608.3699 & 2608.2234 \\ 1652.9124 & 1652.8311\end{array}$

ppm
53
57
47
53
57
53
56
55
56
60
72
47
51
53
60
56
49

\section{Miss Sequence}

- K. DAQPQLEEADDDLDSK. I

1 K. DAQPQLEEADDDLDSKLNYKPPPQK. S

O K. L.NYKPPPQK. S

1 K. ELQEMDKDDESLTK. Y

o K. QDHLTWEWNLAIK. K
1 K. ELQEMDKDDESLTK. Y OX1dation

1 K. KTLLGDVPVVADPTVPNVTVTR. L

O K. TLLGDVPVVADPTVPNVTVTR . L

- R. LSLVCDSAPGPITMDLTGDLEALK . K

1 R. LSLVCDSAP GP I TMDLTGDLEALKK. D

1 R. LSLVCDSAPGPITMDLTGDLEALKK. D

1 K. DTFVLKEGIEYR.V

1 K. VNKDIVSGLK, Y

O K. YVQHTYR. T

O K. ATFMVGSYGPRPEEYEFLTPVEEAPK, G

O K. ATFMVGSYGPRPEEYEFLTPVEEAPK. G

5611 K. SFFTDDDKQDHLTWEWNLAIK. K

(M)
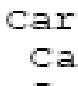

ca

.

。

$\circ$ 


\section{M22}

4700 MS/MS Precursor 1084.67 Spec \#1 MC[BP = 566.3, 1640]

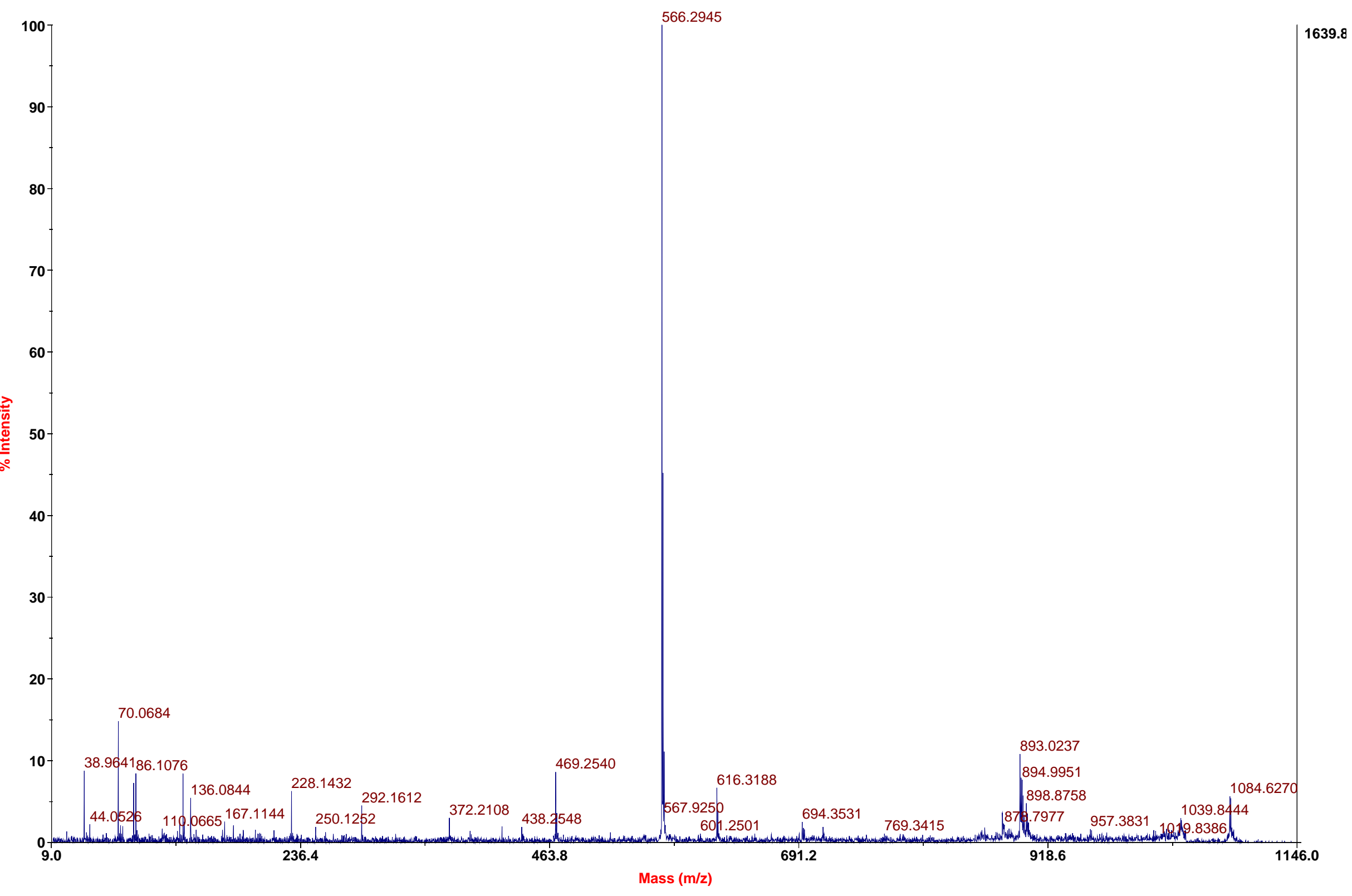




\section{M22}

4700 MS/MS Precursor 1045.62 Spec \#1 MC[BP = 860.9, 1326]

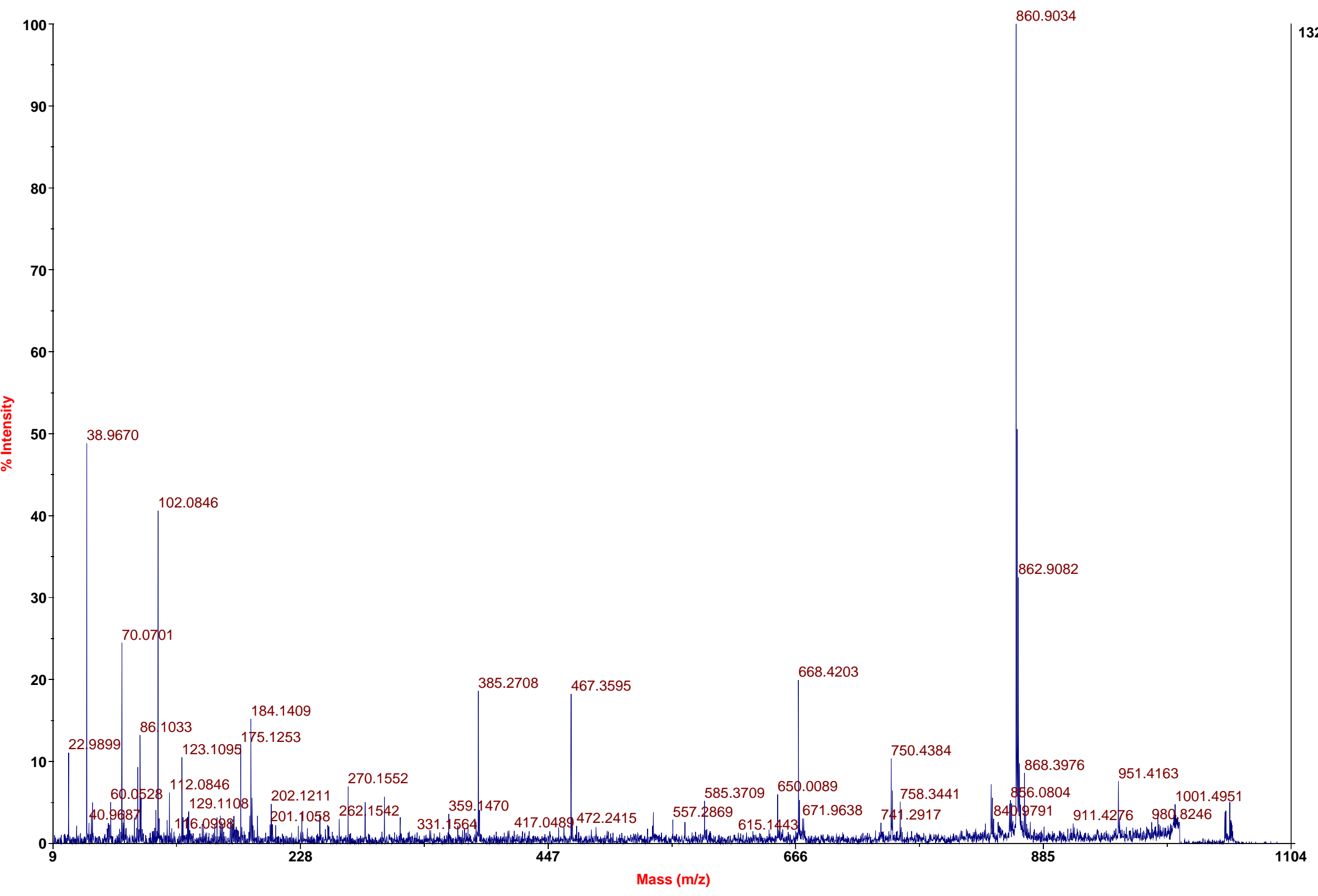




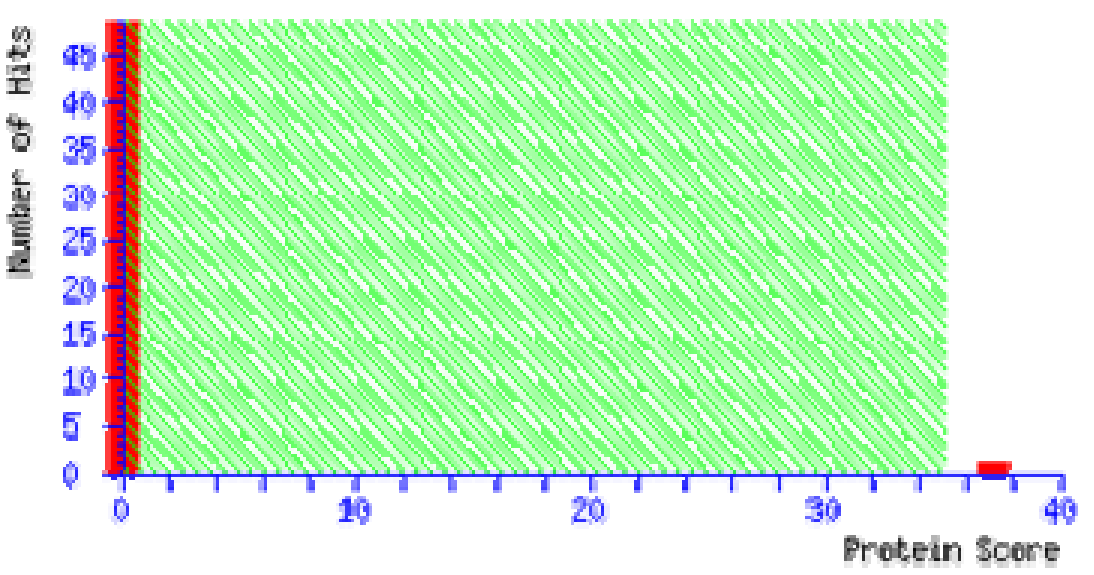

\section{Peptide Summary Report}

Format As Peptide Summary

Significance threshold $\mathrm{p}<0.05$

Standard scoring MudPIT scoring

Show pop-ups Suppress pop-ups
Search Selected $\underline{\text { Help }}$

Max. number of hits AUTO

- Ions score or expect cut-off $0 \quad$ Show sub-sets 0

Sort unassigned Decreasing Score - Require bold red

\section{Error tolerant}
GDIS_MOUSE
Mass: 22705
Score: 37
Matches: $5(0)$
Sequences: $5(0)$ emPAI : 0.65

Rho GDP-dissociation 1nh1b1tor 2 (Rho GDI 2) (Rho-GDI beta) (D4).- Mus musculus (Mouse).

$\square$ check to include this hit in error tolerant search

\begin{tabular}{|c|c|c|c|c|}
\hline \multicolumn{2}{|c|}{ Query } & Observed & $\operatorname{Mr}(\operatorname{expt})$ & $\operatorname{Mr}$ (calc) \\
\hline 目 & $\underline{1}$ & 966.5283 & 965.5210 & 965.4719 \\
\hline & $\underline{6}$ & 1084.6661 & 1083.6588 & 1083.6077 \\
\hline 目 & $\underline{14}$ & 1788.8712 & 1787.8639 & 1787.7697 \\
\hline D & 33 & 2163.3232 & 2162.3159 & 2162.1947 \\
\hline & & $2644 \quad 5061$ & 4088 & 340 \\
\hline
\end{tabular}

$\begin{array}{lcc}\text { ppm } & \text { Miss } & \text { Score } \\ 50.8 & 0 & 31 \\ 47.2 & 0 & 7 \\ 52.7 & 0 & 16 \\ 56.0 & 0 & 30\end{array}$

Expect Rank Unique

$\begin{array}{rrr}0.18 & 1 & \mathrm{U} \\ 30 & 5 & \mathrm{U}\end{array}$

Peptide

K. YVQHTYR. T

K. LNYKPPPQK. S

$9.11 \quad \mathrm{U}$

K. DAQPQLEEADDDLDSK . L

$0.077 \quad 1 \quad \mathrm{U}$

K. TLLGDVPVVADPTVPNVTVTR. L

$563 \quad 1 \quad 0 \quad 0 \quad 24 \quad 1 \quad$

R ISIVCDSAPCD TTMRITCDI.FA I.KK 


\section{Protein View}

Match to: GDIS_MOUSE Score: 37

Rho GDP-dissociation inhibitor 2 (Rho GDI 2) (Rho-GDI beta) (D4) . - Mus musculus (Mouse).

Found in search of Ppw_E6_129990872005.txt

Nominal mass $\left(\mathrm{M}_{\mathrm{r}}\right)$ : 22705; Calculated pI value: 4.97

NCBI BLAST search of GDIS_MOUSE aga1nst nr

unformatted sequence string for pasting into other applications

Taxonony: Mus musculus

Var1able modifications: Carbamidonethy 1 (C), Dean1dated (NQ), oxidation (M)

cleavage by Trypsin: cuts c-term side of KR unless next residue $1 \mathrm{~S}$

Sequence Coverage: 398

Matched peptides shown in Bold Red

1 TEKDAQPQLE EADDDLDSKL NYKPPPQKSL KELQEMDKDD ESLTKYKKTL

51 LGDVPVVADP TVPNVTVTRL SLVCDSAPGP ITMDLTGDLE ALKKDTFVLK

101 EGIEYRVKIN FKVNKDIVSG LKYVQHTYRT GMRVDKATFM VGSYGPRPEE

151 YEFLTPVEEA PKGMLARGTY HNKSFFTDDD KQDHLTWEWN LAIKKDWTE

Show predicted peptides also

\section{Sort Peptides By $\odot$ Residue Number Increasing Mass Decreasing Mass}

\begin{tabular}{|c|c|c|c|c|c|c|}
\hline tart - End & Observed & $\operatorname{Mr}($ expt) & $\operatorname{Mr}(\mathrm{calc})$ & ppm & Miss & Sequence \\
\hline $4-19$ & 1788.8712 & 1787.8639 & 1787.7697 & 53 & 0 & K. DAQPQLEEADDDLDSK. L (Ions score 16) \\
\hline $20-28$ & 1084.6661 & 1083.6588 & 1083.6077 & 47 & 0 & K. LNYKPPPQK.S (Ions score 7) \\
\hline $49-69$ & 2163.3232 & 2162.3159 & 2162.1947 & 56 & 0 & K. TLLGDVPVVADPTVPNVTVTR.L (Ions score 30) \\
\hline $70-94$ & 2644.5061 & 2643.4988 & 2643.3499 & 56 & 1 & R. LSLVCDSAPGPITMDLTGDLEALKK. D Carbamidomethy 1 (C) \\
\hline $123-129$ & 966.5283 & 965.5210 & 965.4719 & 51 & 0 & K. YVQHTYR. T \\
\hline
\end{tabular}


Sample M23

4700 Reflector Spec \#1 MC=>NR(2.00)[BP = 1515.8, 14221]






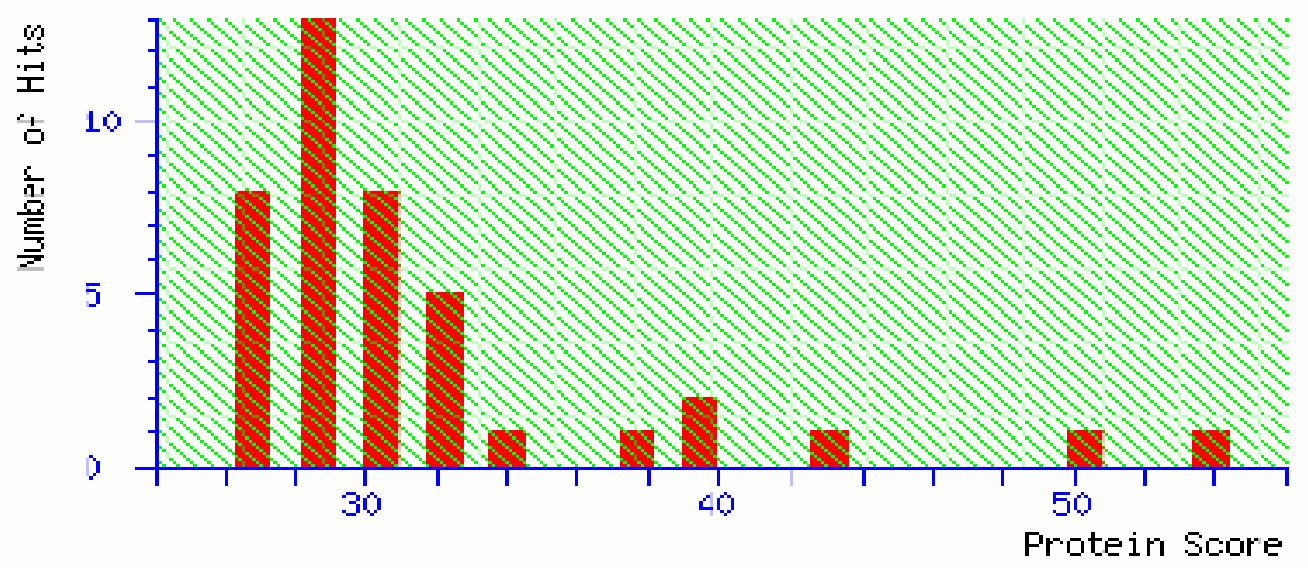

\section{Concise Protein Summary Report}

\begin{tabular}{|l|l|l|l|l|}
\hline Format As & Concise Protein Summary \\
& Significance threshold $\mathrm{p}<$ & Max. number of hits \\
0.05 & AUTO
\end{tabular}

Re-Search All

Search Unmatched

1.

\begin{tabular}{l}
$\frac{\text { AAF99333 }}{\text { AF289664 }}$ NID: 115840 Score: 54 \\
AAH39162 \\
BC039162 NID: - Mus musculus \\
AAH53048 \\
\hline
\end{tabular}
Expect: 0.38 Matches: 29
Expect: 1.2 Matches: 26
Expect: 1.3 Matches: 26




\section{Protein View}

Match to: AAF99333 Score: 54 Expect: 0.38

\section{AF289664 NID : - Mus musculus}

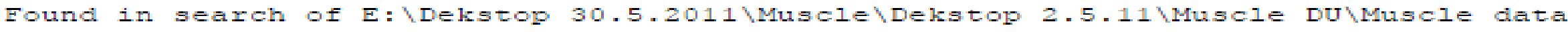
$\backslash$ sample M23 \pmf_G6_129990866106.txt

Nominal mass $\left(M_{Y}\right)$ : 115840; Calculated pI value: 6.07

NCBI BLAST search of AAF99333 against nr

Unformatted sequence string for pasting into other applications

Taxonomy: Mus musculus

Links to retrieve other entries containing this sequence from NCBI Entrez:

(no taxonomy information for this entry)

(no taxonomy information for this entry)

Variable modifications: Carbamidomethyl (C), Deamidated (NQ), Oxidation

Cleavage by Trypsin: cuts C-term side of KR unless next residue is $P$

Number of mass values searched: 83

Number of mass values matched: 29

Sequence Coverage: 248

Matched peptides shown in Bold Red

1 MQKPSGLKPP GRGGKHSSPV

51 GPSSSGAATT VSEKPGPKA.

101 ETQFAPGQWA GVVLDDPVGK

151 TAEGSGSDTH SVESITAQNL

201 NESGSNLSDS GSVKRGDKDL

251 GVELDEPLGK NDGAVAGTRY

301 KTKRMAMGVS ALTHSPSSSS

351 KISGTTALQE ALKEKQQHIE

401 KAQHEQYVAE AEEKLQRARI

451 EEESITKGDI ETQTQLEHAR

501 AEKSRVLQLE EELSLRRGEI

551 LSASKEHQRD STLLQDKYEH

601 QQATTENMGL MDNWKSKLDS

651 KALVEGIKME HQLELGNLQA

701 QQHWREQLEE QASQHRLELQ

751 EFLKEQISLA EKKMILDYEML

801 LCSAQHSHVI ESSDLSEETI

851 RAQVSALENK CKSGEKKIDS

901 QELLRKERSI NELRVLILEA

951 IRGLREKLTG LDKEKSLSEQ

1001 ALNQAQQVER LVEALRGCSD
GRPSVGSASS EVGDDFLGDE NDGAVGGVRY SLHSGTATPE HLGDRVLVGG FQCPRKFGLF ISSVSSVASS QLLAERDLER LVENVRKEKV IGELEQSLLL EELQHCLLQS MLKTYQTEVD LASDHQKSLE KHDLETAMHG EAQDQCRDAQ QRAEAQSRQE RMKETVEGLQ LLKEKRRLEA NRHSPGPERD RRYSLIDPAS RTQTISNSGS
SVVASTSGSK EGSPLHKQAS VVGERVWVING VRPGVVQYLG FECPALQGIF TRPSKLTRQP LTGRVIPLRE SVLNSSVKTG TKTGVVRYVG ETDFAKGEWC APIHKVIRIG FPSTSPAKAK VGGRPSRSGL LTETSSRYAR AEVAKATSHI CEVEKEIALI DLSNQLEEER RKVEDLQFRV EKAQAERLLR ELADNRITTV GPPPADHPEA AETLRLRERL KLRAANEKYA QEVADLKAKV DLKATLNSGP GAQQKEIGEI KEKEGLRQKL QEVQEELAGL LRAQELEGLD VEYRGQAQAI AERLREKLLV AENRLQAAES DKLNKRDKEV TALTSQMDMI. ELEAVSRKTH DASGQLVHIS LSREVHKAEW RIKEQKLKDD PPELLKLQHQ LVSTEDALRD ANGIHQPDKA HKQEDKH 


\section{M23}

4700 MS/MS Precursor 976.488 Spec \#1 MC[BP = 343.2, 2779]

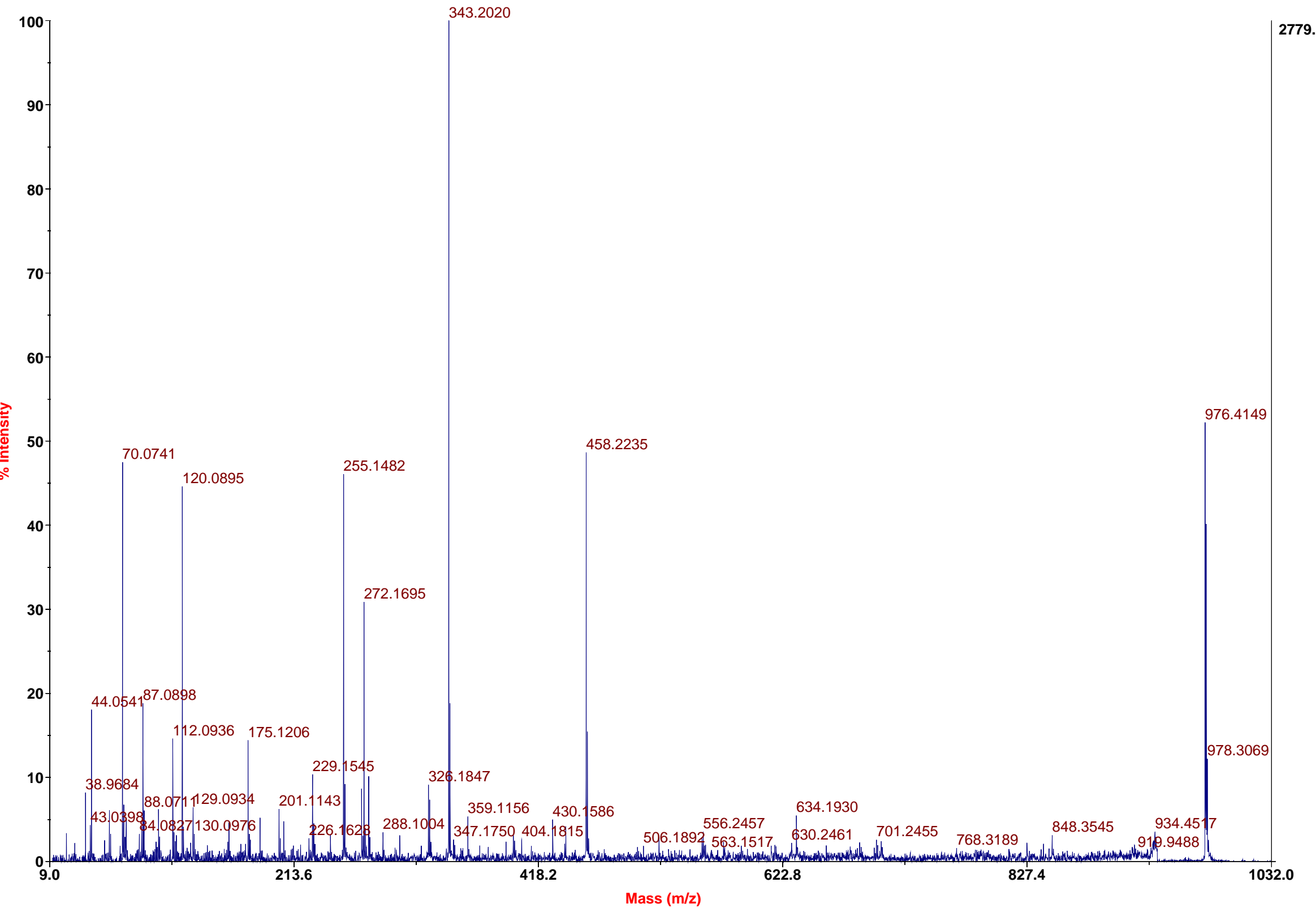




\section{M23}

4700 MS/MS Precursor $1045.61 \mathrm{Spec} \mathrm{\# 1} \mathrm{MC[BP} \mathrm{=} \mathrm{860.9,} \mathrm{2224]}$

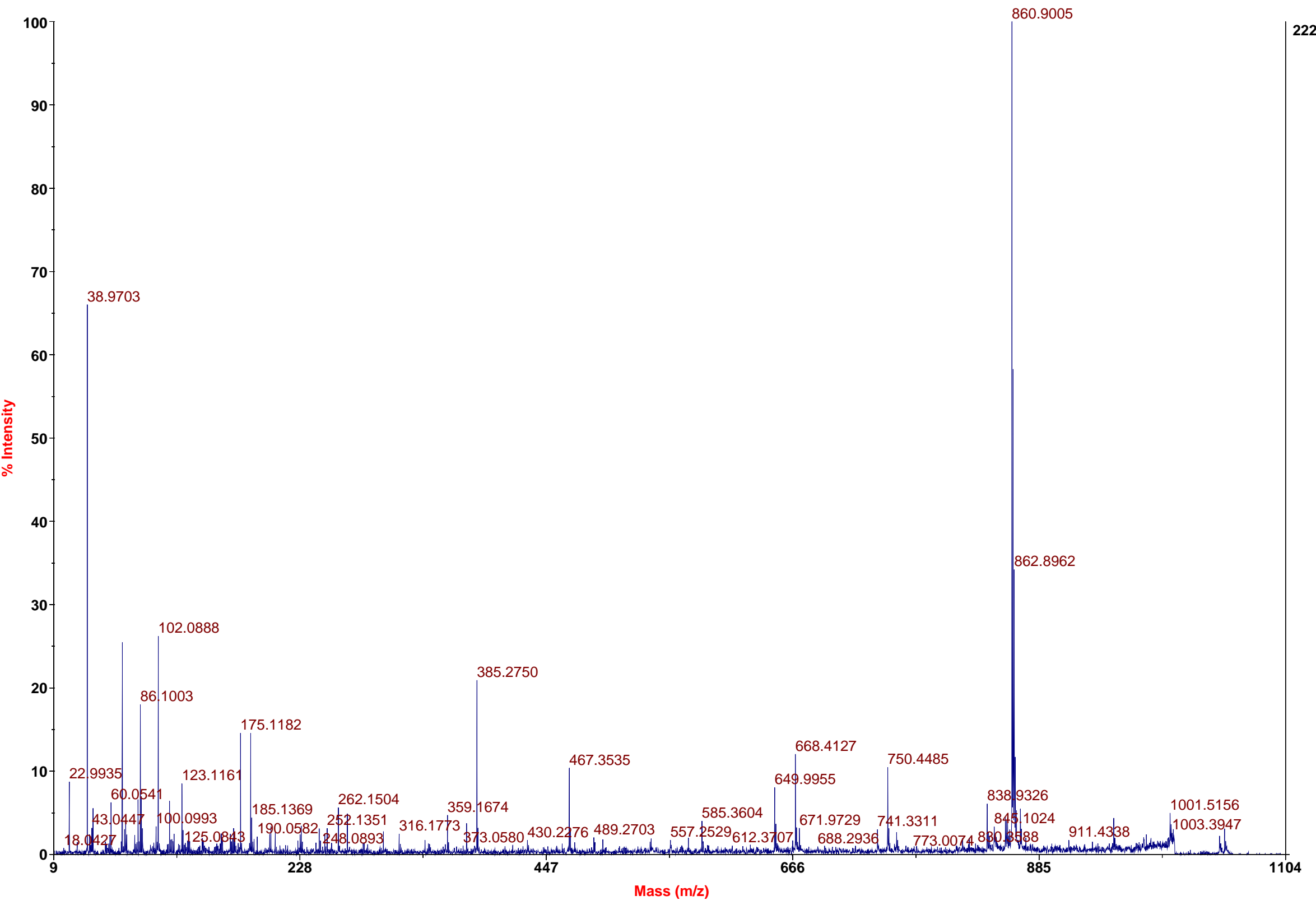






\section{Peptide Summary Report}

Format As Peptide Summary

Significance threshold $p<0.05$

Standard scoring MudPIT scoring $\odot$ Ions score or expect cut-off 0

Show pop-ups Suppress pop-ups Sort unassigned Decreasing Score
Help

Max. number of hits AUTO

Show sub-sets 0

Require bold red

\section{Select All}

\section{Select None}

Search Selected

\section{Error tolerant}

1. CAA27396 Mass: 39161 Score: 55 Matches: 5(3) Sequences: 5(3) emPAI: 0.59 MMACTBR2 NID: - Mus musculus

check to 1nclude this hit in error tolerant search

\begin{tabular}{|c|c|c|c|c|c|c|c|c|c|}
\hline Query & Observed & $\mathrm{Mr}$ (expt) & $\mathrm{Mr}$ (calc) & ppm & Miss & Score & Expect & Rank & Unique \\
\hline D & 945.5899 & 944.5826 & 944.5444 & 40.5 & 0 & 42 & 0.014 & 1 & $\mathrm{U}$ \\
\hline$\underline{8}$ & 1171.6173 & 1170.6100 & 1170.5638 & 39.5 & 0 & 42 & 0.017 & 1 & \\
\hline$\underline{12}$ & 1354.6886 & 1353.6813 & 1353.6161 & 48.2 & 1 & 27 & 0.6 & 1 & \\
\hline$\underline{14}$ & 1515.8197 & 1514.8124 & 1514.7419 & 46.6 & 0 & 18 & 4.4 & 1 & \\
\hline 26 & 1954.1547 & 1953.1474 & 1953.0571 & 46.2 & 0 & 37 & 0.032 & 1 & \\
\hline
\end{tabular}

Peptide

R.AVFPSIVGR.S

R. HQGVMVGMGQK. D

K. DSYVGDEAQSKR. G

K. IWHHTFYNELR.V

R. VAPEEHPVLLTEAPLNPK. A 


\section{Protein View}

Match to: CAA27396 score: 55

MMACTBR2 NID: - Mus musculus

Found in search of ppw_G6_129990872106.txt

Nom1nal mass $\left(\mathrm{M}_{\mathrm{r}}\right)$ : 39161; Calculated pI value: 5.78

NCBI BLAST search of CAA27396 aga1nst $\mathrm{nr}$

unformatted sequence string for pasting 1nto other applications

Taxonony: Mus musculus

Links to retrieve other entries containing this sequence from NCBI Entrez:

(no taxonomy information for this entry)

Var1able modifications: Carbanidomethy 1 (C), Dean1dated (NQ), ox1dation (M)

cleavage by Trypsin: cuts C-term side of KR unless next residue is $P$

sequence coverage: $17 \%$

Matched peptides shown in Bold Red

1 PRAVFPSIVG RSRHQGVMVG MGQKDSYVGD EAQSKRGILT LKYPIEHGIV

51 TNWDDMEKIW HHTFYNELRV APEEHPVLLT EAPLNPKANR EKMTQIMFET

101 FNTPAMYVAI QAVLSLYASG RTTGIVMDSG DGVTHTVPIY EGYALPHAIL

151 RLDLAGRDLT DYLMKILTER GYSFTTTAER EIVRDIKEKL CYVALDFEQE

201 MATAASSSSL EKSYELPDGQ VITIGNERFR CPEALFQPSF LGMESCGIHE

251 TTFNSIMKCD VDIRKDLYAN TVLSGGTTMY PGIADRMOKE ITALAPSTMK

301 IKIIAPPERK YSVWIGGSIL ASLSTFQQMN ISKQEYDESG PSIVHRKCF

Show predicted peptides also

Sort Peptides By Residue Number Increasing Mass Decreasing Mass

$\begin{array}{rrrr}\text { Start - End } & \text { Observed } & \text { Mr (expt) } & \text { Mr (calc) } \\ 3-11 & 945.5899 & 944.5826 & 944.5444 \\ 14-24 & 1171.6173 & 1170.6100 & 1170.5638 \\ 25-36 & 1354.6886 & 1353.6813 & 1353.6161 \\ 59-69 & 1515.8197 & 1514.8124 & 1514.7419 \\ 70-87 & 1954.1547 & 1953.1474 & 1953.0571\end{array}$

ppm Miss Sequence

410 R. AVFPSIVGR.S (Ions score 42)

390 R. HQGVMVGMGQK. D (Ions score 42)

481 K. DSYVGDEAQSKR.G (Ions score 27)

470 K. IWHHTFYNELR.V (Ions score 18)

460 R. VAPEEHPVLLTEAPLNPK.A (IONS score 37) 
Sample M24

4700 Reflector Spec \#1 MC=>NR(2.00)[BP = 1475.8, 16515]

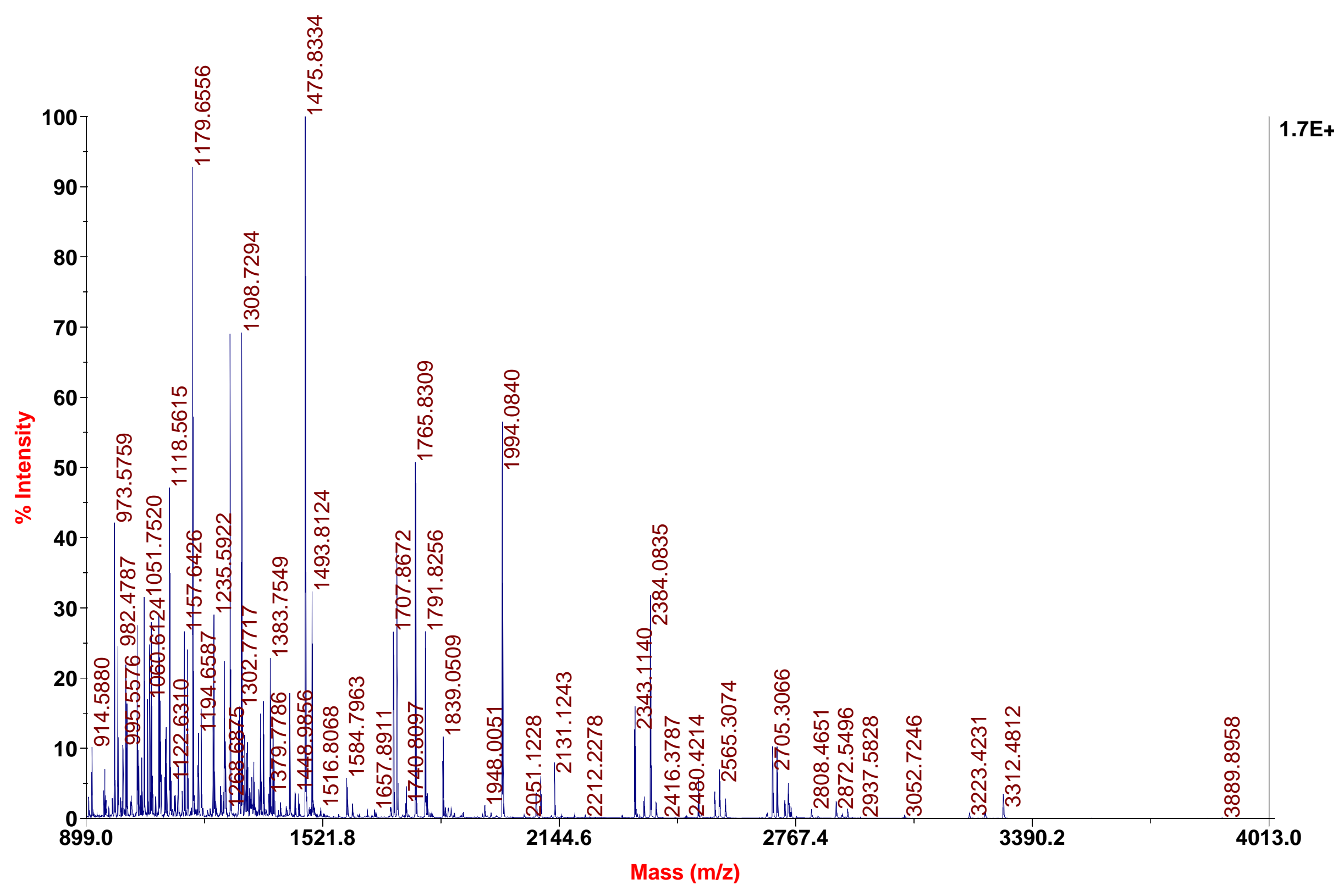




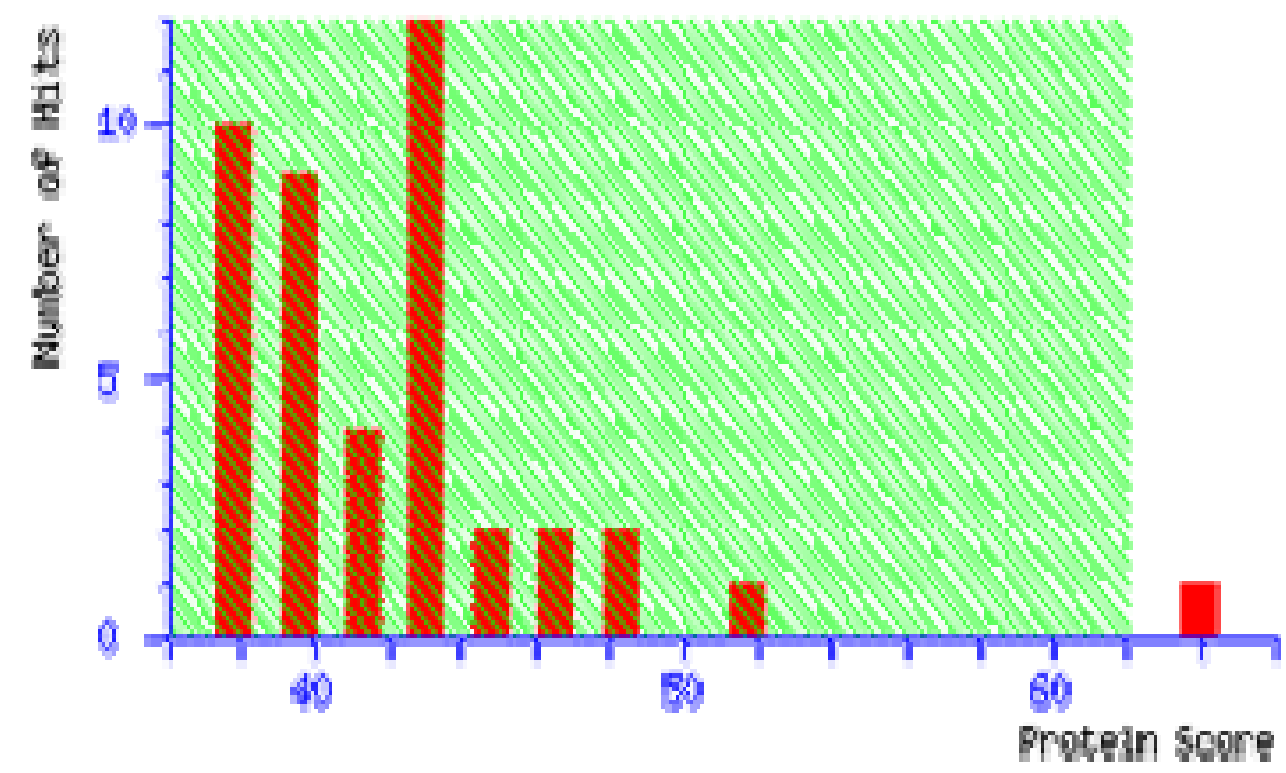

\section{Concise Protein Summary Report}
Format As
Concise Protein Summary
$\underline{\text { Help }}$
Significance threshold $\mathrm{p}<0.05$
Max. number of hits AUTO

\section{Re-Search All}

Search Unmatched

1. Q6zOK2 MoUSE Mass: 191193 Score: 64 Expect: 0.038 Matches: 65 MKIAa0051 prote1n (Fragnent).- Mus musculus (Mouse).

QBCGH5 MOUSE Mass: 106421 Score: 51 Expect: 0.72 Matches: 45 Iggapl prote1n (Fragnent).- Mus musculus (Mouse). 


\section{Protein View}

Match to: $96 z Q k 2$ MOUSE score: 64 Expect: 0.038 Found $1 \mathrm{n}$ search of pmf_H6_129990866107.txt

Nominal mass $\left(M_{Y}\right)=191193$; calculated pI value: 6.15

NCRI BLAST search of o6zok2 MoUse against nr
Unformatted sequence string for pasting 1nto other applications

Iaxonomy: Mus musculus

Links to retrieve other entries containing this sequence from NceI Entrez

(no taxonomy 1 nformation for this entry)

Var1able modifications: Carbamidomethyl (C), Deanidated (Ne), oxidation

cleavage by Irypsin: cuts c-term side of KR unless next residue 15 f

Number of mass values searched: 180

Number of mass values matched: 65

sequence coverage: $37 \%$

Matched peptides shown in Bold Red

1 LILLRSPASS FSLFFLtGDL DSAAMSAAEE 51 TAEEMDERRR QNVAYEYLCH LEEAKRWMEA 101 YLAKLGNFFS PKVVSLKKIY DREQTRYKAT
151 GLPKIFYFET IDIYDRKNMP RCIYCIHALS 201 FTEEEINNMIK IELEKYGIOM PAFSKIGGIL 251 AIDRRVAADT FTALKNPNAM LVNLEEGIAP 301 TENSDRERDV TEELLTQAEI OGNVNKVNTS 351 ALQSLALGLR GLQTQNSDWY MKQLQSDLQO 401 AANSAAQQYQ RRIAAVAAIN AAIQKGIAEK 451 DLYQKELATL QQQSPEHSLT HPELTVAVEM 501 KQLSSSVTGL INIEEENCQR YLDELMKLKA 551 HVNLVVHEEH ERILAIGLIN EALDEGDAOK 601 QHYQDTLIRA KREKAOETOD ESAVLWLDEI 651 ISAINEAVDS GDVGRTLSAL RSPDVGLYGV 701 AAGDNNSKWV KHWVKGGYHY YHNLETOAGG

751 SSISGVTAAY NREOLWIANE GIITKLOACC

801 PAITCIOSON RGYKOKKAYO DRIMYLHSHK

851 DRLOYFFDHI NDIIKIOAFI RANKALHSHK

901 DQSDQDFQEE LDLMKMREEV ITLIRSNQQL

951 LQDVVSHSKK LTKKNKEQLS DMMMINKQKG

1001 YLLQTNPTYL AKLIFQMPQN KSTKFMDSVI

1051 QTALQEEIKS KVDQIQEIVT GNPTVIKMVV

1101 KEIMDDKSIN IKTDPVDIYK SWVNQMESQT

1101 KEIMDDKSIN IKTDPVDIYK SWVNQMESOT

1151 DRTRLDNSIR NMRAVTDKFL SAIVSSVDKI

1251 SIAKMIQHAA SNKMIFIGDNA HLSIINEYIS

1301 DKFNVDEYSD LVTLTRPVIY ISIGEIINTH

1351 LIDDLGEVPT IESLIGESCG NSNDPNKEAL

1401 AEMDARTILI NTKRLIVDVI RFQPGETLTE

1451 RAIRDAKTPD KMKKSKPMKE DNNLSLQEKK

1501 RYQELINDIA KDIRNQRRYR QRRKAELVKL

1551 YKSYIKTCLD NLASKGKVSK KPREMKGKKS

1601 IEDLQANQFK NVIFEIGPTE EVGDFEVKAK

VDGLGVVRPH YGSVLDNERI CLGEDLFPTT ELEEGLRNG GLHFRHTDNV IONLNAMDE LYLFKLGLAF QIQDLYGKVD ANELSVDEAA LHAAVIAINE TYQDVLYQAK QDKMTNAKNF SALANISLAL EQGCAVTLLK KRQSGQTDFL QKEEVOAGVD TVLELMNPEA OLPQVYPFAA LSSVALINFA LESGDMTTVW OAHAENNAFI TWNDIOACVD ILQALQIPAA KLEGVLAEVA QGGIWQSNKD IOEAORFALG IPECGETYRS DLAEAKKKRL WAEPFDFVON SVOLSREEIO RGYLVROEFR SRIMNEIKKOI DEVVKIQSLA RMHQARKRYR ENDINIMDIK IGLIVKNHIT GIKAISKEKR EKIEAYOHIE ETIYNYASNO REEYTIT SENRGARGON AIROIIAPVV GEASKI.PYDV TPEOAISHEE PYGMRFIAKV LKDSLHEKEP DILSAGGQ LTIDORENEG QST TLLLDHODAI AEEHNDIHE TIN I TYTELKL TELGTVDEKN Q KRISLITIAA RIHEKGULLE LHYQDLLQL $\kappa$

Show predicted peptides also

\section{Sort Peptides By}

- Residue Number

Increasing Mass

Decreasing Mass

$50-58$

$50-59$

$61-75$

$119-126$

$127-135$
$168-185$

\section{Obse rved} 1109.5443 1265.7042 1868.0181 1081.5437 2315.2419

\section{Mr (expt)} 1108.5370 1264.6969 1867.0108 1080.5364 1091.5561
2314.2346
Mr (calc)

$1108-4706$

$1264 \cdot 5717$

1866.8458

1080.5200

2314.1424 ppm

60

99

15

40
Miss Sequence

- R. ITAEEMDER. R Oxidation (M)

R. ONVAYEYLCHLERAK. R Carbamido

1 K. IYDREQTR. $Y$ Deamidated (NQ)

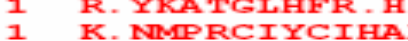




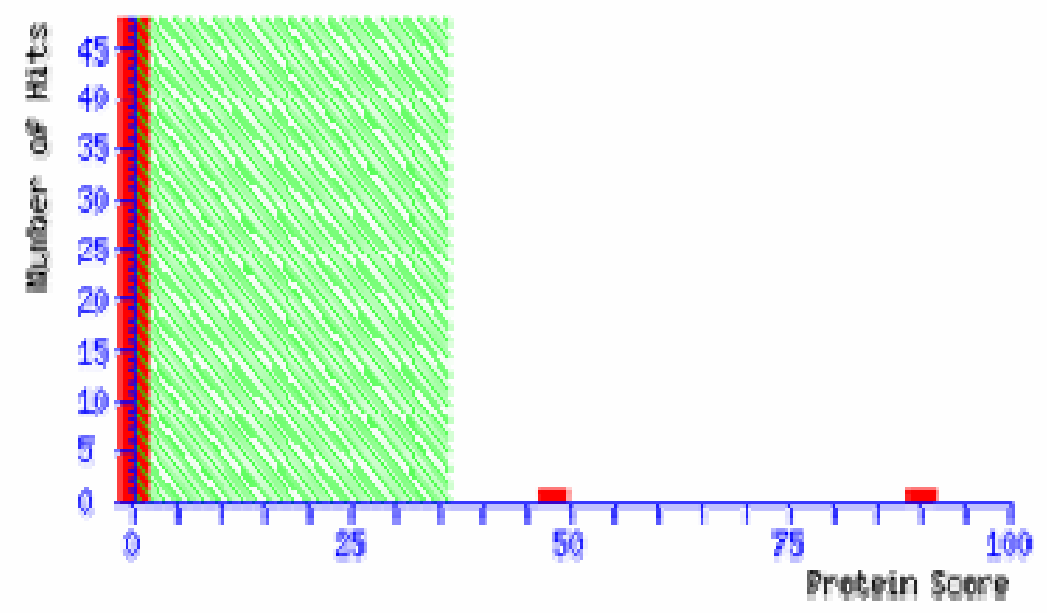

\section{Peptide Summary Report}

Format As Peptide Summary

Significance threshold $p<0.05$

Standard scoring $\bigcirc$ MudPIT scoring $\odot$ Ions score or expect cut-off 0

Show pop-ups 9 Suppress pop-ups Sort unassigned Decreasing Score
Help

Max. number of hits AUTO

Show sub-sets 0

Require bold red

\section{Select All Select None Search Selected $\square$ Error tolerant}

1. O3TK56 MOUSE Mass: 10117 Score: 90 Matches: 2(1) Sequences: 2(1) emPAI: 0.70

Blastocyst blastocyst CDNA, RIKEN full-length enriched l1brary, clone:I1C0034H10 product:act1n related prote1n 2/

$\square$ check to include th1s hit in error tolerant search

\begin{tabular}{|c|c|c|c|c|c|c|c|c|c|c|}
\hline Query & Observed & $\operatorname{Mr}$ (expt) & $\operatorname{Mr}(\mathrm{calc})$ & ppm & Miss & Score & Expect & Rank & Unique & Peptide \\
\hline 2 & 1070.6798 & 1069.6725 & 1069.6244 & 45.0 & 0 & 16 & 2.5 & 1 & $\mathrm{U}$ & K.ALAAGGVGSIVR.V \\
\hline$\underline{44}$ & 2131.1230 & 2130.1157 & 2130.0018 & 53.5 & 0 & 90 & $3 e-07$ & 1 & U & K. GFESPSDNSSAVLLQWHEK . A \\
\hline
\end{tabular}




\section{Protein View}

Match to: Q3TK56_MOUSE Score: 90

Blastocyst blastocyst CDNA, RIKEN full-length enriched library, clone:I1C0034H10 product:actin related protein $2 / 3$ Found 1n search of Ppw_H6_129990872207.txt

Nominal mass $\left(\mathrm{M}_{\mathrm{r}}\right)$ : 10117; Calculated pI value: 9.79

NCBI BLAST search of Q3TK56_MOUSE against nr

Unformatted sequence string for pasting into other applications

Taxonony: Mus musculus

Links to retrieve other entries containing this sequence from NCBI Entrez:

(no taxonomy information for this entry)

Var1able modifications: Carbanidomethyl (C), Deamidated (NQ), oxidation (M)

cleavage by Trypsin: cuts C-term side of KR unless next residue is $P$

sequence Coverage: 328

Matched peptides shown in Bold Red

1 ALKNPPINTK SQAVKDRAGS IVLKVLISFK ANDIEKAVQS LDKNGVDLLM

51 KYIYKGEESP SDNSSAVLLQ WHEKALAAGG VGSIVRVLTA RKTV

Show predicted peptides also

Sort Peptides By 9 Residue Number Increasing Mass Decreasing Mass

$\begin{array}{crrrrrll}\text { Start - End } & \text { Observed } & \operatorname{Mr}(\text { expt) } & \operatorname{Mr}(\mathrm{calc}) & \text { ppm } & \text { Miss } & \text { Sequence } \\ 56-74 & 2131.1230 & 2130.1157 & 2130.0018 & 53 & 0 & \text { K. GFESPSDNSSAVLLQWHEK. A } & \text { (Ions Score 90) } \\ 75-86 & 1070.6798 & 1069.6725 & 1069.6244 & 45 & 0 & \text { K. ALAAGGVSIVR.V } & \text { (Ions score 16) }\end{array}$


Sample M25

4700 Reflector Spec \#1 MC=>NR(2.00)[BP = 2785.5, 12229]

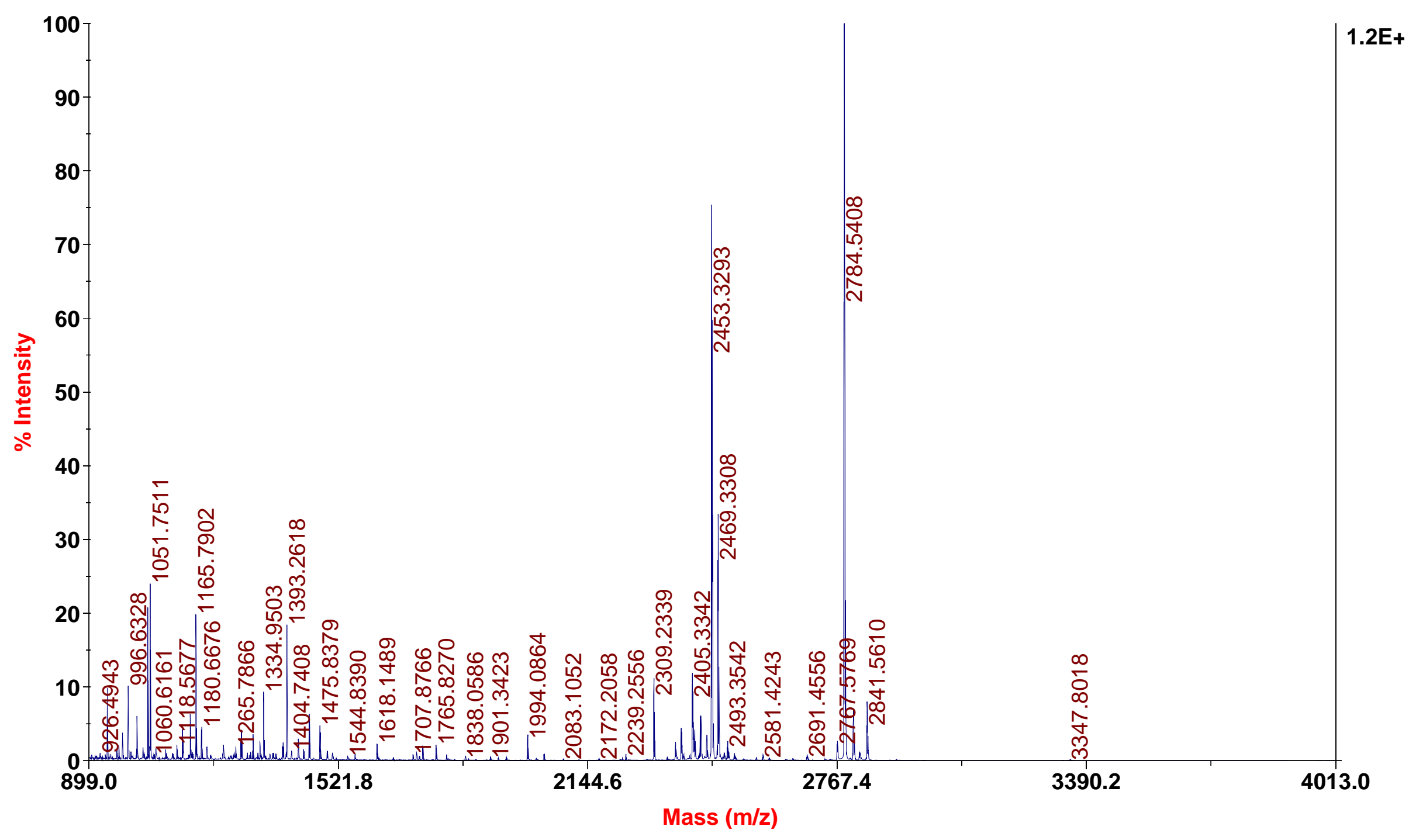


$4700 \mathrm{MS} / \mathrm{MS}$ Precursor $1045.61 \mathrm{Spec} \# 1 \mathrm{MC}[\mathrm{BP}=860.9,1151]$


$\operatorname{Mass}(\mathrm{m} / \mathrm{z})$ 
4700 MS/MS Precursor 996.633 Spec \#1 MC[BP = 216.1, 1537]

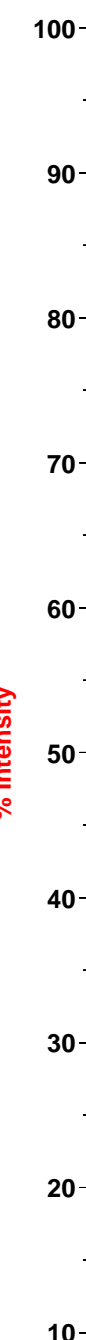

$10-$

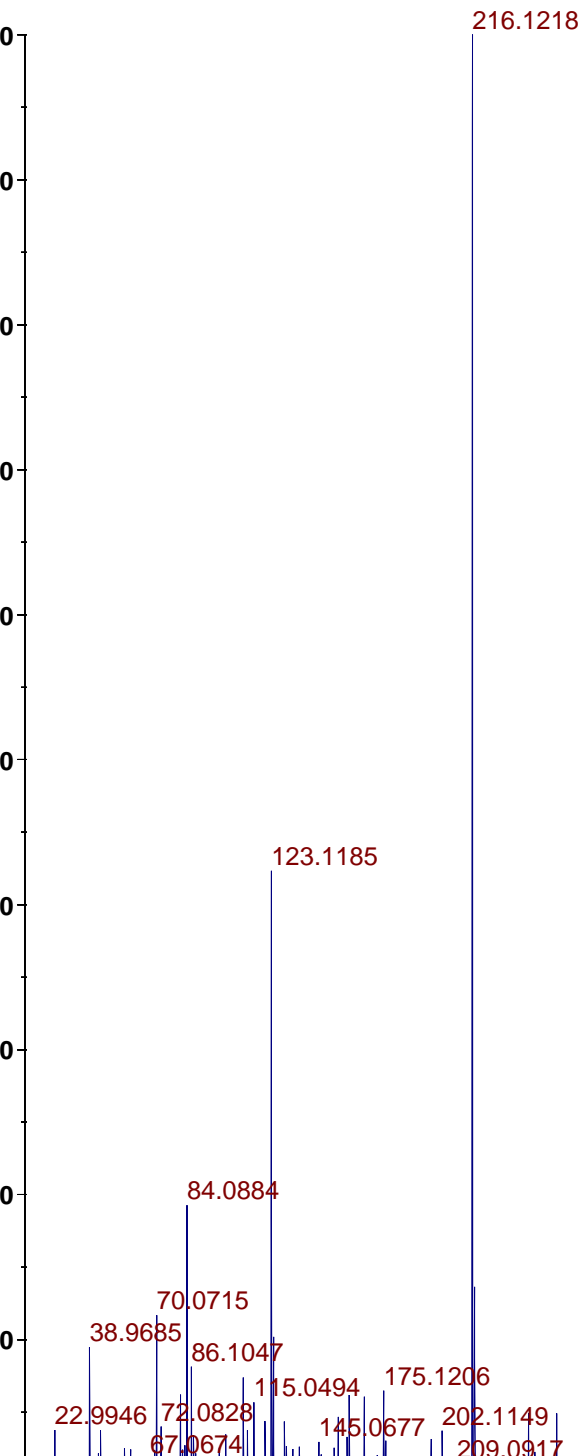

16.1218

499.2855

284.1811

357.2191406 .2918459 .247

$\begin{array}{rr}217.8 & 426.6\end{array}$

581.3555

782.4185 
Sample M26

4700 Reflector Spec \#1 MC=>NR(2.00)[BP = 1298.8, 57153]








\section{Concise Protein Summary Report}

Format As Concise Protein Summary . Help

Significance threshold $p<0.05 \quad$ Max, number of hits AUTO

\section{Re-Search All Search Unmatched}

1. Mixture 1 Total score: 181 Expect: $7.3 \mathrm{e}-14$ Matches: 45 Components (only one family member shown for each component):

Q9D1R6 MOUSE Mass: 32804 Score: 167 Expect: 1.8e-12 Matches: 35

18-day embryo whole body CDNA, RIKEN full-length enriched l1brary, clone:1100001k11 product:tropomyosin 2, beta, full 1nsert sequ Q9CY74 MOUSE Mass: 17645 Score: 53 Expect: 0.43 Matches: 11

8 days embryo whole body CDNA, RIKEN full-length enriched l1brary, clone:5730592G18 product:RIKEN CDNA 5730592G18.- Mus musculus 


\section{Protein View}

Match to: Q9D1R6_Mouse score: 167 Expect: $1.8 e-12$

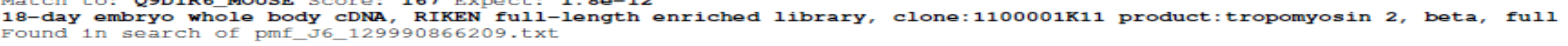

Nominal mass $\left(M_{x}\right)=32804$; Calculated PI value: 4.64

NCBI BLAST search of O9D1R6 MOUSE aga1nst nr

Unformatted sequence string for pasting 1nto other applications

Taxonomy: Mus musculus
Links to retrieve other

作

ion for this entry)

var1able mod1f1cat1ons: Carbamidomethyl (C), Deamidated (No), Ox1dat1on. (M Cleavage by Trypsin: cuts C-term slde of KR unless next residue $1 \mathrm{~s} P$

Number of mass values searched: 129

sequence coverage: 70 s

Matched peptides shown in Bold Red

1 MDAIKKKMQM LKLDKENAID RADEAEADKK QAEDRCKQLE EEQQALQKKL

51 KGTEDEVEKY SESVKDAQEK LEQAEKKATD AEADVASLNR RIQLVEEELD

101 RAQERLATAL QKLEEAEKAA DESERGMKVI ENRAMKDEEK MELQEMQLKE

151 AKHIAEDSDR KYEEVARKLV ILEGELERSE ERAEVAESTC FDIERSVAKIV

251 KTIDDLEDEV YAOKMKYKAI SEELDNALND ITSI.

Show predicted peptides also

\begin{tabular}{|c|c|c|c|c|c|}
\hline \multicolumn{3}{|c|}{ Sort Peptides By } & \multicolumn{2}{|c|}{ - Residue Number } & Increasing \\
\hline $\begin{array}{r}\text { Start } \\
13\end{array}$ & $\underline{-}$ & $\begin{array}{l}\text { End } \\
21\end{array}$ & $\begin{array}{l}\text { Observed } \\
1073.6161\end{array}$ & $\begin{array}{r}\text { Mr (expt) } \\
1072.6088\end{array}$ & $\begin{array}{c}\operatorname{Mr}(\text { calc }) \\
1072.5513\end{array}$ \\
\hline 31 & - & 37 & 906.4929 & 905.4856 & 905.4025 \\
\hline 36 & - & 48 & 1576.7727 & 1575.7654 & 1575.7450 \\
\hline 36 & - & 48 & 1631.8989 & 1630.8916 & 1630.7984 \\
\hline 38 & - & 48 & 1343.7493 & 1342.7420 & 1342.6728 \\
\hline 38 & - & 49 & 1471.8601 & 1470.8528 & 1470.7678 \\
\hline 38 & - & 49 & 1475.8446 & 1474.8373 & 1474.7038 \\
\hline 50 & - & 59 & 1147.6428 & 1146.6355 & 1146.5768 \\
\hline 52 & - & 65 & 1599.8319 & 1598.8246 & 1598.7311 \\
\hline 60 & - & 70 & 1283.6857 & 1282.6784 & 1282.6041 \\
\hline 66 & - & 76 & 1288.7073 & 1287.7000 & 1287.6306 \\
\hline 77 & - & 90 & 1460.8223 & 1459.8150 & 1459.7267 \\
\hline 78 & - & 90 & 1332.7152 & 1331.7079 & 1331.6317 \\
\hline 78 & - & 91 & 1488.8296 & 1487.8223 & 1487.7328 \\
\hline 91 & - & 101 & 1399.8350 & 1398.8277 & 1398.7467 \\
\hline 92 & - & 101 & 1243.7239 & 1242.7166 & 1242.6456 \\
\hline 92 & - & 105 & 1727.9966 & 1726.9893 & 1726.8849 \\
\hline 113 & - & 125 & 1476.7725 & 1475.7652 & 1475.6739 \\
\hline 141 & - & 149 & 1149.6221 & 1148.6148 & 1148.5569 \\
\hline 141 & - & 149 & 1165.6195 & 1164.6122 & 1164.5518 \\
\hline 141 & - & 152 & 1510.8165 & 1509.8092 & 1509.7054 \\
\hline 153 & - & 160 & 942.4771 & 941.4698 & 941.4203 \\
\hline 153 & - & 161 & 1070.5800 & 1069.5727 & 1069.5152 \\
\hline 168 & - & 178 & 1298.8428 & 1297.8355 & 1297.7605 \\
\hline 169 & - & 178 & 1170.7357 & 1169.7284 & 1169.6656 \\
\hline 169 & - & 182 & 1671.9833 & 1670.9760 & 1670.8839 \\
\hline 179 & - & 189 & 1234.6641 & 1233.6568 & 1233.5837 \\
\hline 183 & - & 198 & 1749.9827 & 1748.9754 & 1748.8138 \\
\hline 190 & - & 198 & 1092.5413 & 1091.5340 & 1091.4805 \\
\hline 190 & - & 205 & 1817.9924 & 1816.9851 & 1816.9240 \\
\hline 190 & - & 205 & 1875.0626 & 1874.0553 & 1873.9455 \\
\hline 206 & - & 217 & 1340.7412 & 1339.7339 & 1339.6619 \\
\hline 218 & - & 226 & 1182.6132 & 1181.6059 & 1181.5452 \\
\hline 234 & - & 244 & 1308.7366 & 1307.7293 & 1307.6106 \\
\hline 252 & - & 264 & 1538.8053 & 1537.7980 & 1537.7148 \\
\hline
\end{tabular}

Decreasing Mass

ppm
54
92
13
57
52
58
91
51
58
58
54
61
57
60
58
57
60
62
50
52
69
53
54
58
54
55
59
92
49
34
59
54
51
91
54

$\begin{array}{ll}1 & \text { Sequence } \\ \text { K. LDKENA IDR. A }\end{array}$

1 K. OAEDRCK.Q Carbam1domethyl (C)

1 R. CKQLEEEQQALQK.K 2 Deamidated (NQ)

R. CKQLEEEQQALQK.K Carbam1domethyl (C)

K. QLEEEQOALOK . K

$\mathrm{K}$. QLEEEQQALQKK. I

K. OLEEEQQALOKK. I 4 Deamidated (NO)

K. LKGTEDEVEK . Y

K. GTEDEVEKYSESVK. D

K. YSESVKDAQEK. L

K. DAQEKLEQAEK. K

K. ATDAFADVASILNR. R

R. RIQLVEEELDR. A

R. IOLVEEELDR. A

R. IOLVEEELDRAQER. I

K. LEEAEKAADESER. G

K. MELAEKAADESER . E

Oxidatton (m)

K. MELQFMQLKEAK. H Deamidated (NQ); 2 Oxidation (M)

K. HIAEDSDR. K

K. HIAEDSDRK. Y

R. KLVILEGELER. S

K. LVILEGELER.S

K. LVILEGELERSEER. A

R. SEERAEVAESK. C

R. AEVAESKCGDLEEELK. I

K. CGDLEELIK.I Carbamidomethyl (C)

K. CGDLEEELKIVTNNLK.

K. CGDLEEELKIVTNNLK.S Carbam1domethyl (C)

K. SLEAQADKYSTK . F

K. EDKYEEEIK. L

1
K. EAETRAEFAER. S
K. TIDDLEDEVYAQK. M 
4700 MS/MS Precursor 1243.72 Spec \#1 MC[BP = 175.1, 6847]

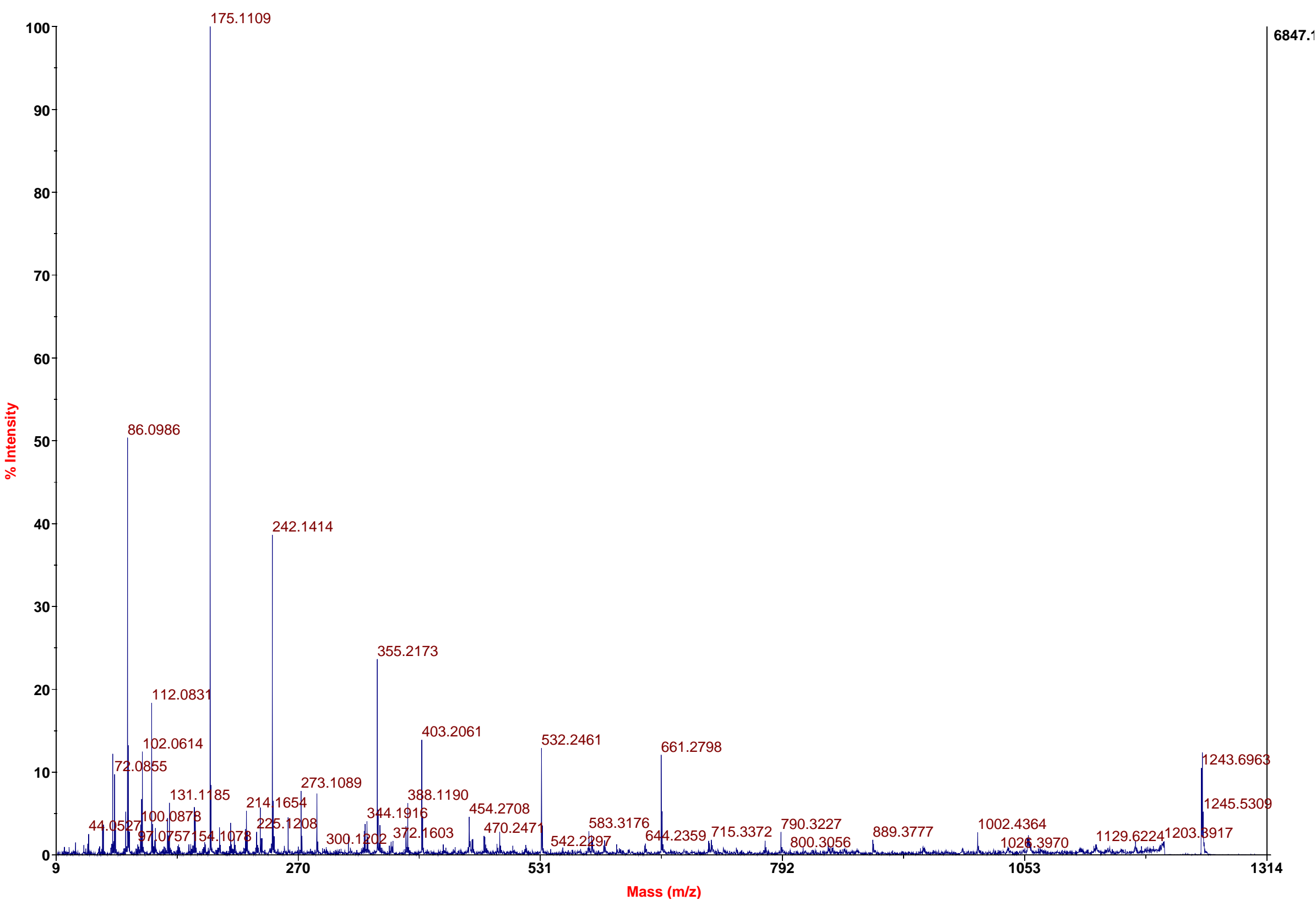


4700 MS/MS Precursor 1170.74 Spec \#1 MC[BP = 86.1, 5248]

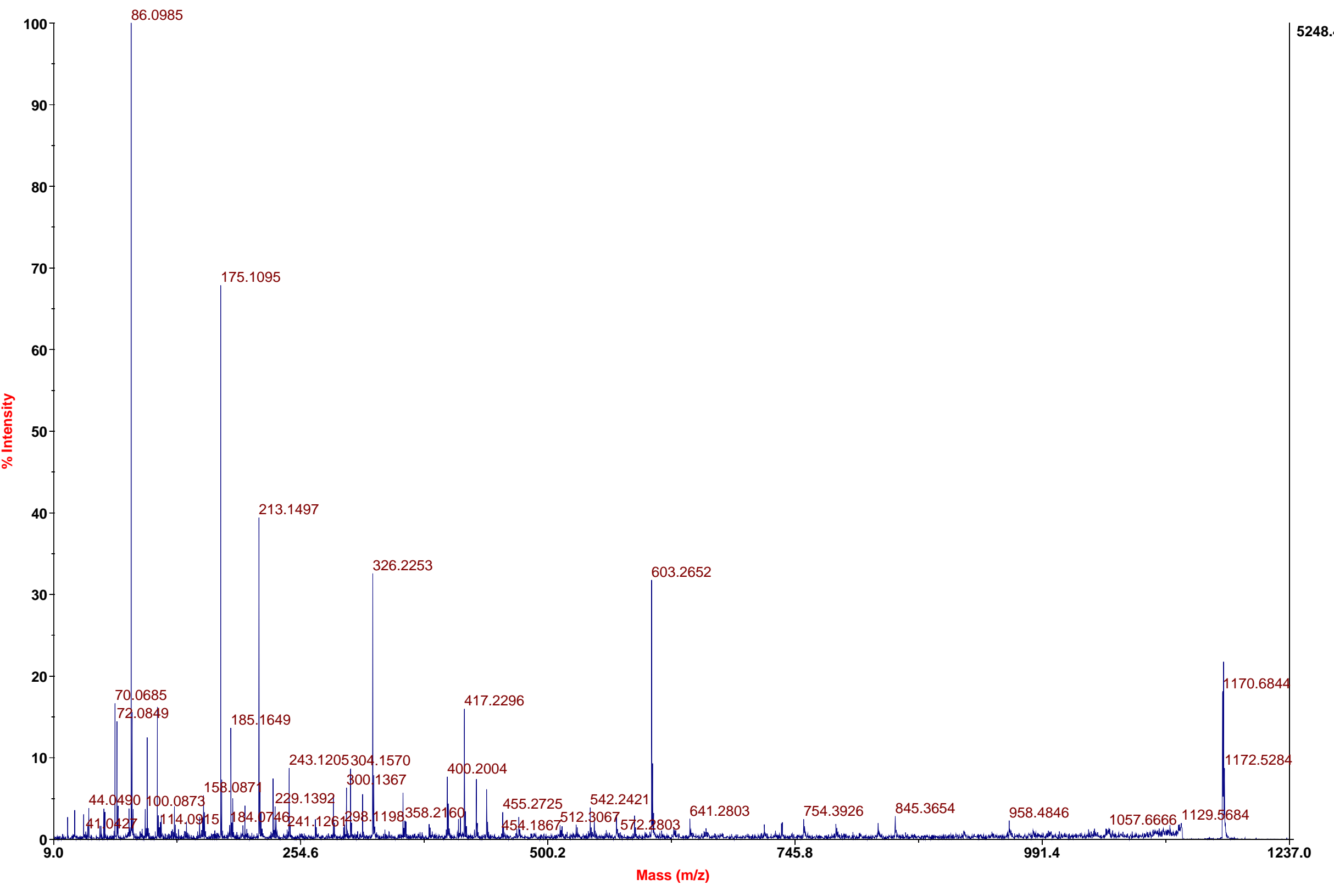




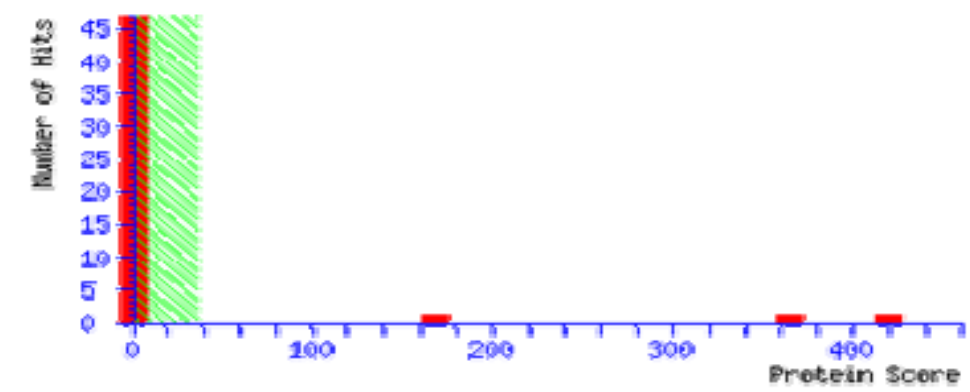

\section{Peptide Summary Report}

Format As Peptide Summary

Significance threshold $\mathrm{p}<0.05$

Standard scoring $\odot$ MudPIT scoring $\odot$ Ions score or expect cut-off 0

Show pop-ups 9 Suppress pop-ups $\odot$ Sort unassigned Decreasing Score $\underline{\text { Help }}$

Show sub-sets 0

Require bold red $\Pi$

\section{Select All Select None Wrror tolerant}

1. TMRBB Mass: 32817 Score: 420 Matches: $15(12)$ Sequences: $15(12)$ emPAI: 8.90 tropomyosin beta cha1n, skeletal muscle [validated] - rabb1t (tentative sequence)

П check to 1 nclude this hit in error tolerant search

\begin{tabular}{|c|c|c|c|c|}
\hline \multicolumn{2}{|c|}{ Query } & Observed & $\operatorname{Mr}($ expt $)$ & $\operatorname{Mr}(\mathrm{calc})$ \\
\hline จ & 1 & 942.4771 & 941.4698 & 941.4203 \\
\hline & $\underline{2}$ & 1073.6161 & 1072.6088 & 1072.5513 \\
\hline & $\underline{3}$ & 1092.5413 & 1091.5340 & 1091.4805 \\
\hline & $\underline{5}$ & 1170.7357 & 1169.7284 & 1169.6656 \\
\hline & $\underline{6}$ & 1182.6132 & 1181.6059 & 1181.5452 \\
\hline & 7 & 1243.7239 & 1242.7166 & 1242.6456 \\
\hline & $\underline{8}$ & 1298.8428 & 1297.8355 & 1297.7605 \\
\hline 目 & $\underline{9}$ & 1343.7493 & 1342.7420 & 1342.6728 \\
\hline & $\underline{10}$ & 1399.8350 & 1398.8277 & 1398.7467 \\
\hline 7 & $\underline{11}$ & 14 & 3150 & 267 \\
\hline$\sqrt{1}$ & 12 & 1476.7725 & 1475.7652 & 1475.6739 \\
\hline & 13 & 1488.8296 & 1487.8223 & 1487.7328 \\
\hline & $\underline{14}$ & 1538.8053 & 1537.7980 & 1537.7148 \\
\hline 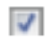 & $\underline{16}$ & 1631.8989 & 1630.8916 & 1630.7984 \\
\hline & 17 & 1727.9966 & 1726.9893 & 1726.8849 \\
\hline
\end{tabular}

$\begin{array}{lrrrrr}\text { ppm } & \text { Miss } & \text { Score } & \text { Expect } & \text { Rank Unique } \\ 52.7 & 0 & 46 & 0.0052 & 1 & \\ 53.7 & 1 & 37 & 0.048 & 1 & \\ 49.1 & 0 & 55 & 0.001 & 1 & \text { U } \\ 53.7 & 0 & 76 & 2.3 e-06 & 1 & \\ 51.4 & 1 & 35 & 0.091 & 1 & \\ 57.2 & 0 & 65 & 7 e-05 & 1 & \\ 57.8 & 1 & 82 & 3.1 e-07 & 1 & \\ 51.5 & 0 & 57 & 0.0005 & 1 & \\ 58.0 & 1 & 16 & 4 & 1 & \\ 60.5 & 1 & 72 & 1.6 e-05 & 1 & \\ 61.9 & 1 & 74 & 1.4 e-05 & 1 & \\ 60.2 & 1 & 33 & 0.14 & 1 & \\ 54.1 & 0 & 85 & 1 e-06 & 1 & \text { U } \\ 57.1 & 1 & 85 & 8.1 e-07 & 1 & \\ 60.5 & 1 & 51 & 0.0017 & 1 & \end{array}$

\section{Peptide}

K. HIAEDSDR. K

K. LDKENA IDR . A

K. CGDLEEELK.I + Carbamidomethyl (C)

K. LVILEGELER. S

K. EDKYEEEIK . L

R. IQLVEEELDR. A

R. KLVILEGELER. S

K. QLEEEQQALQK . K

R. RIQLVEEELDR. A

K. KATDAEADVASLNR. R

K. LEEAEKAADESER , G

K. ATDAEADVASLNRR. I

K. TIDDLEDEVYAQK . M

R. CKKQLEEEQQALQK.K + Carbamidomethyl (C)

R. IQLVEEELDRAQER. L 


\section{Protein View}

Match to: TMRBB score: 420

tropomyosin beta chain, skeletal muscle [validated] - rabbit (tentative sequence)

Found 1n search of ppw_J6_129990872509.txt

Nom1nal mass $\left(M_{x}\right)$ : 32817 ; Calculated PI value: 4.66

NCBI BLAST search of TMRBB aga1nst nr

unformatted sequence string for pasting 1nto other applications

Taxonomy: oryctolagus cun1culus

Links to retrieve other entr1es conta1n1ng this sequence from NCBI Entrez:

(no taxonomy 1nformation for this entry)

(no taxonomy information for this entry)

(no taxonomy information for this entry)

(no taxonomy 1nformation for this entry)

(no taxonomy 1nformation for this entry)

(no taxonomy information for this entry)

(no taxonomy information for this entry)

(no taxonomy information for this entry)

(no taxonomy information for this entry)

(no taxonomy 1nformation for this entry)

Var1able mod1f1cat1ons: Carbam1domethyl (C), Deam1dated (NQ), ox1dat1on (M)

cleavage by Trypsin: cuts C-term side of KR unless next residue $1 \mathrm{~s} P$

sequence Coverage: 408

Matched peptides shown in Bold Red

1 MDAIKKKMQM LKLDKENAID RAEQAEADKK QAEDRCKQLE EEQQALQKKL

51 KGTEDEVEKY SESVKDAQEK LEQAEKKATD AEADVASLNR RIQLVEEELD

101 RAQERLATAL OKLEEAEKAA DESERGMKVI ENRAMKDEEK MELOEMOLKF

151 AKHIAEDSDR KYEEVARKLV ILEGELERSE ERAEVAESKC GDLEEELKIV

201 TNNLKSLEAQ ADKYSTKEDK YEEEIKLLEE KLKEAETRAE FAERSVAKLE

251 KTIDDLEDEV YAQKMKYKAI SEELDNALND ITSL

Show predicted peptides also

\section{Sort Peptides By \\ a Residue Number \\ $\odot$ Increasing Mass $\cap$ Decreasing Mass}

Start - End
$13-21$
$36-48$
$38-48$
$77-90$
$78-91$
$91-101$
$92-101$
$92-105$
$113-125$
$153-160$
$168-178$
$169-178$
$190-198$
$218-226$
$252-264$

Observed
1073.6161
1631.8989
1343.7493
1460.8223
1488.8296
1399.8350
1243.7239
1727.9966
1476.7725
942.4771
1298.8428
1170.7357
1092.5413
1182.6132
1538.8053

\section{Mr (expt)} 1072.6088

1630.8916

1342.7420

1459.8150

1487.8223

1398.8277

1242. 7166

1726.9893

1475.7652

941.4698

1297.8355

1169.7284

1091.5340

1181.6059

1537.7980

\section{Mr (calc)}

1072.5513

1630.7984

1342.6728

1459.7267

1487.7328

1398.7467

1242.6456

1726.8849

1475.6739

941.4203

1297.7605

1169.6656

1091.4805

1181.5452

1537.7148

ppm
54
57
52
61
60
58
57
60
62
53
58
54
49
51
54

\section{Miss Sequence}

1 K. LDKENAIDR.A (IOnS score 37)

1 R. CKQLEEEQQALQK $\mathrm{K}$ Carbam1domethy

K. QLEEEQQALQK. K (IONS score 57)

K. KATDAFADVASLNR. $R$ (IOnS SCOre 72)

K. ATDAEADVASLNRR. I (Ions score 33)

R. RIQLVEEELDR. A (IONS score 16)

R. IQLVEEELDR.A (I ONS score 65)

R. IQLVEEELDRAQER. L (IONS score 51)

K. LEEAEKAADESER.G (I

K. HIAEDSDR. K (IONS Score 46)

R. KLVILEGELER. S (IONS score 82)

K. LVILEGELER.S (IONS score 76)

K. CGDLEEELK. I Carbamidomethyl (C) (Ions score 55)

K. EDKYEEEIK. I (Ions score 35)

0 K. TIDDLEDEVYAQK. M (IONS score 85)
(C) (Ions score 85)

$=$ 
Sample M27

\section{Reflector Spec \#1 MC=>NR(2.00)[BP = 1243.7, 68319]}

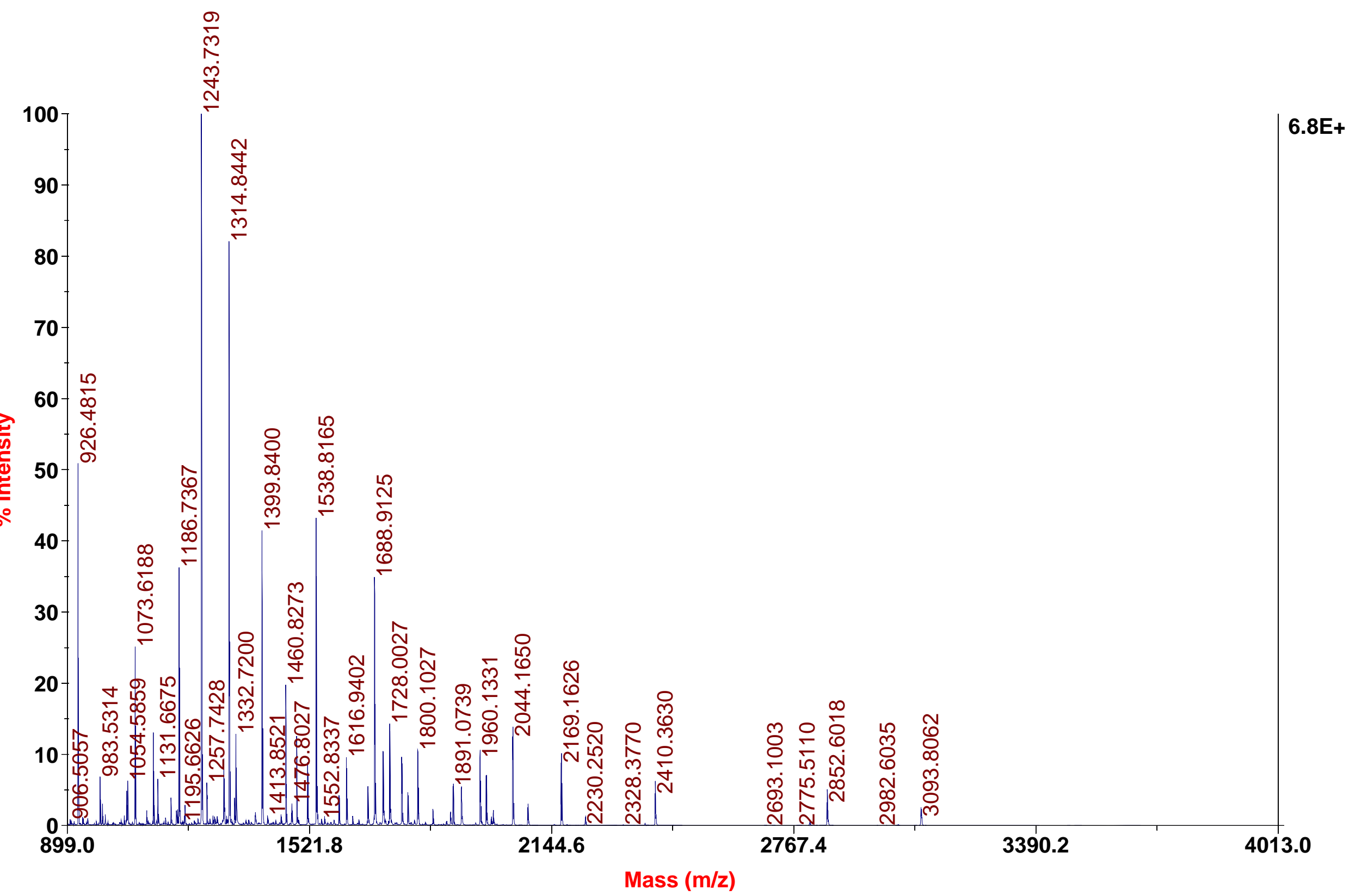




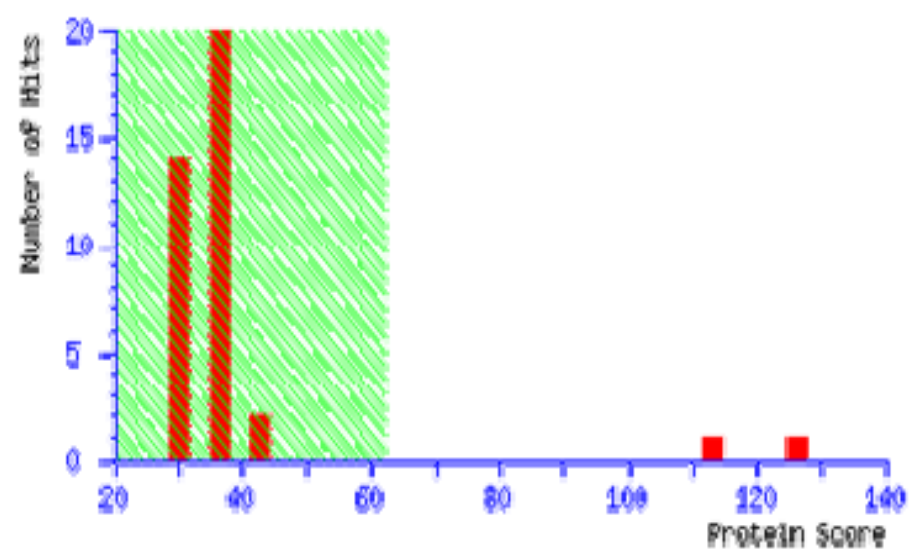

\section{Concise Protein Summary Report}

Format As Concise Protein Summary .

Significance threshold $p<\overline{0.05}$ Max. number of hits AUTO

\section{Re-Search All Search Unmatched}

1. TMRBA Mass: 32661 Score: 126 Expect: $2.3 \mathrm{e}-08$ Matches: 31

tropomyosin alpha cha1n, card1ac and skeletal muscle [val1dated] - rabb1t

Q8BSH3 MOUSE Mass: 32679 Score: 111 Expect: $7.3 e-07$ Matches: 29

12 days embryo male wolffian duct 1ncludes surround1ng reg1on cDNA, RIKEN full-length enr1ched 11brary, clone:6720477I24

A39816 Mass: 32689 Score: 94 Expect: $3.8 \mathrm{e}-05$ Matches: 28

tropomyosin 2, fibroblast - rat

Q99PB8 MOUSE Mass: 1525 Score: 33 Expect: 48 Matches: 3

Adenos1ne k1nase (EC 2.7.1.20) (Fragment).- Mus musculus (Mouse). 
Match to: TMRRA score: 126 Expect: $2.3 e-08$

tropomyosin alpha chain, cardiac and skeletal muscle [validated] - rabbit

Found 1n search of pmf_k6_129990866210.txt

Nominal mass $\left(M_{x}\right): 32661$; Calculated PI value: 4.69

NCBI BLAST search of TMRBA against ni

unformatted sequence string for pasting 1nto other applications

Links to retrieve other entr1es conta1ning this sequence from NCBI Entrez:

(no taxonomy 1 nformation for this entry)

(no taxonomy information for this entry)

(no taxonomy information for this entry)

(no taxonomy information for this entry)

(no taxonomy 1nformation for this entry)

(no taxonomy 1 nformation for this entry)

(no taxonomy information for this entry)

(no taxonomy information for this entry)

(no taxonomy 1nformation for this entry)

Var1able modif1cations: Carbamidomethyl (C), Deamidated (NQ), Ox1dation

Cleavage by Trypsin: cuts C-term S1de of KR unless next residue $1 \mathrm{~s} P$

Number of mass values matched: 31

sequence Coverage: 69 s

Matched peptides shown in Bold Red

1 MDAIKKKMMM LKLDKENALD RAEQAEADKK AAEDRSKQLE DELVSLQKKL

51 KGTEDELDKY SEALKDAQEK LELAEKKATD AEADVASLNR RIQLVEEELD

101 RAQERLATAL OKLEEAEKAA DESERGMKVI ESRAQKDEEK MEIQEIQLKE

151 AKNIKSIRA

201 TNNLKSLEAQ AEKYSQKEDK YEEEIKVLSD KLKEAETRAE FAERSVTKLE

Show predicted peptides also

Sort Peptides By $\square$ Residue Number Increasing Mass

\begin{tabular}{|c|c|c|}
\hline & - & \\
\hline 13 & - & 21 \\
\hline 16 & - & 29 \\
\hline 22 & - & 30 \\
\hline 36 & - & 48 \\
\hline 38 & - & 48 \\
\hline 52 & - & 65 \\
\hline 66 & - & 76 \\
\hline 78 & - & 90 \\
\hline 78 & - & 91 \\
\hline 91 & - & 101 \\
\hline 92 & - & 101 \\
\hline 92 & - & 105 \\
\hline 137 & - & 149 \\
\hline 141 & - & 149 \\
\hline 141 & - & 149 \\
\hline 141 & - & 152 \\
\hline 141 & - & 152 \\
\hline 141 & - & 152 \\
\hline 153 & - & 160 \\
\hline 153 & - & 161 \\
\hline 168 & - & 178 \\
\hline 169 & - & 178 \\
\hline 169 & - & 182 \\
\hline 190 & - & 198 \\
\hline 190 & - & 205 \\
\hline 199 & - & 213 \\
\hline 218 & - & 226 \\
\hline 234 & - & 244 \\
\hline 252 & - & 264 \\
\hline 269 & - & 284 \\
\hline 269 & - & 284 \\
\hline
\end{tabular}

Observed
1073.6185
1560.8030
989.5439
1516.9116
1301.7655
1597.8563
1273.7345
1332.7179
1488.8333
1399.8395
1243.7312
1728.0024
1632.9154
1131.6670
1147.6593
1460.8265
1475.8484
1476.8030
926.4818
1054.5851
1314.8416
1186.7365
1671.9976
1120.5782
1891.0714
1648.9156
1182.6207
1308.7417
1538.8136
1758.9299
1774.9274

\section{Mr (expt)} 1072.6112 1559.7957 158.5366 1515.9043 1596.8490 1596.8490 1272.7272 1331.7106 1398.8322 1242.7239 1726.9951 1631.9081 1130.6597 1146.6520 1459.8192 1474.8411 1475.7957 925.4745 1053.5778 1313.8343 1670.9903 1670.9903 1890.0641 1890.0641 1181.6134 $1307 \cdot 7344$ 1537.8063 1757.9226
1773.9201
Decreasing Mass

ppm
56
57
55
59
54
61
56
59
63
61
63
64
62
52
49
41
48
28
53
55
60
58
64
53
65
53
58
95
60
62
63
988.4825 1515.8144 1596.7519 1272.6561 1331.6317 1487.7328 1398.7467 1242.6456 1726.8849 1631.8076 1130.6005 1146.5954 1459.7592 1474.7701 925.4253 053.5203 1313.7554 1670.8839 1119.5117 1889.9404 1647.8203 1307.6106 1537.7148 1773.8091

\section{Miss Sequence}

DEADK. K Deam1dated (NO)

$\mathrm{K}$. OIFDEIVSLOK. $\mathrm{K}$

K. GTEDELDKYSEALK. D

K. DAQEKLEIAEK. $K$

K. ATDARADVASINR. R

R. RIQLVEEELDR. A

R. IQLVEEELDRAQER. I

K. DEEKMEIQEIQLK . E

K. MEIQEIQIK .

K.MEIQEIQLK.E Ox1dation (M)

K. MEIQEIQLKEAK.H Deam1dated (NO)

K. MEIQEIQLKEAK. F

K.MEIQEIQLKEAK.H Deam1dated (NQ); Ox1dation (M)

K. HIAEDADR. K

R. HIAEDADRK. $Y$

R. KLVIIESDLER. A

K. CAELERELK. T Carbamidomethy 1 (C)

K. CAELEEELKTVTNNLK.S Carbam1domethy 1

$K$ TVTNNLKSIEAOAEK Y $Y$ Deam1dated (NO)

K. EDKYEEEIK . V

K. EAETRAEFAER. S

K. SIDDLEDELYAQK . I

K. AISEELDHA LNDMTSI. -
O K. AISEELDHA LNDMTSI. -

oxidation (M) 
$4700 \mathrm{MS} / \mathrm{MS}$ Precursor 1314.84 Spec \#1 MC[BP = 86.1, 3006]




4700 MS/MS Precursor 926.482 Spec \#1 MC[BP = 175.1, 3809]

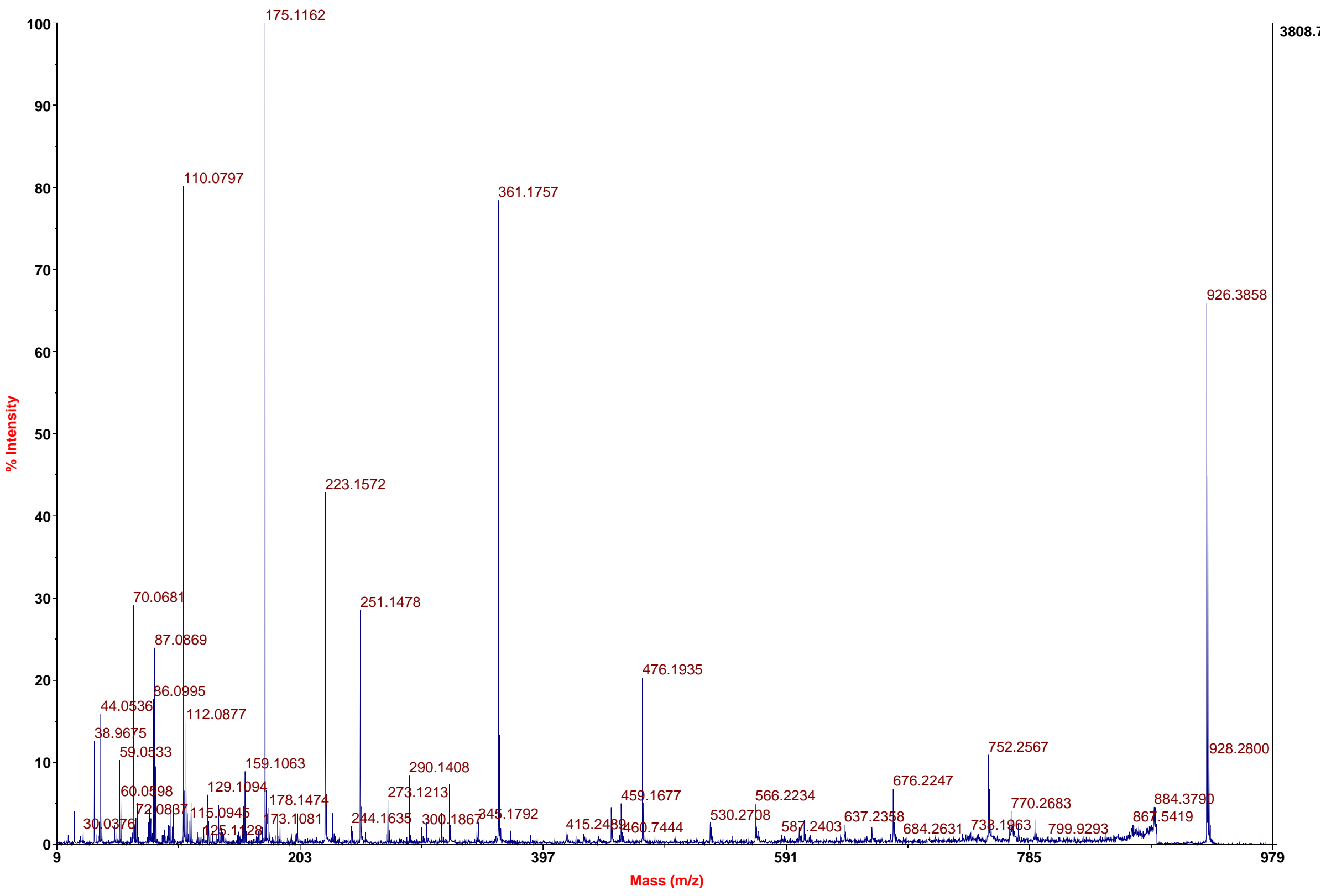




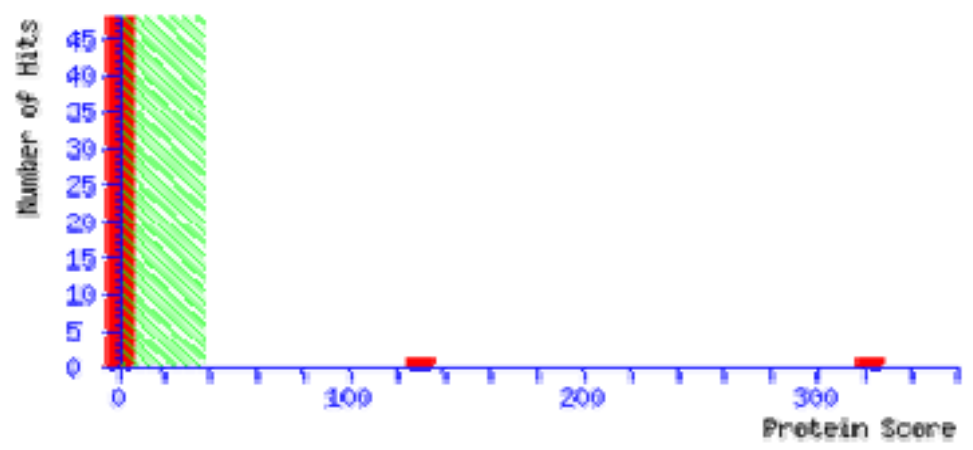

\section{Peptide Summary Report}

\section{Format As Peptide Summary}

Significance threshold $\mathrm{p}<0.05$

Standard scoring $\bigcirc$ MudPIT scoring

Show pop-ups 9 Suppress pop-ups $\cap$ Sort unassigned Decreasing Score $\underline{\text { Help }}$

Max. number of hits AUTO

Show sub-sets 0

Require bold red $\square$

\section{Select All Select None Error tolerant}

1. TMRBA Mass: 32661 Score: 323 Matches: $12(11)$ Sequences: 12 (11) emPAI: 5.50

tropomyosin alpha cha1n, card1ac and skeletal muscle [validated] - rabb1t

П Check to 1nclude this h1t in error tolerant search

\begin{tabular}{|c|c|c|c|c|}
\hline \multicolumn{2}{|c|}{ Query } & Observed & Mr (expt) & $\operatorname{Mr}(\mathrm{calc})$ \\
\hline 同 & 1 & 926.4818 & 925.4745 & 925.4253 \\
\hline 同 & $\underline{2}$ & 1073.6185 & 1072.6112 & 1072.5513 \\
\hline 同 & $\underline{3}$ & 1120.5782 & 1119.5709 & 1119.5117 \\
\hline 同 & $\underline{4}$ & 1186.7365 & 1185.7292 & 1185.6605 \\
\hline 同 & $\underline{5}$ & 1243.7312 & 1242.7239 & 1242.6456 \\
\hline 同 & $\underline{6}$ & 1314.8416 & 1313.8343 & 1313.7554 \\
\hline 同 & 7 & 1399.8395 & 1398.8322 & 1398.7467 \\
\hline 同 & $\underline{8}$ & 1460.8265 & 1459.8192 & 1459.7267 \\
\hline 同 & $\underline{9}$ & 1488.8333 & 1487.8260 & 1487.7328 \\
\hline 同 & $\underline{10}$ & 1538.8136 & 1537.8063 & 1537.7148 \\
\hline 同 & $\underline{14}$ & 1728.0024 & 1726.9951 & 1726.8849 \\
\hline 同 & $\underline{15}$ & 1758.9299 & 1757.9226 & 1757.8141 \\
\hline
\end{tabular}

$\begin{array}{lcrrcc}\text { ppm } & \text { Miss } & \text { Score } & \text { Expect } & \text { Rank Unique } \\ 53.2 & 0 & 61 & 0.00017 & 1 & \mathrm{U} \\ 55.9 & 1 & 49 & 0.0032 & 1 & \mathrm{U} \\ 52.9 & 0 & 46 & 0.0079 & 1 & \mathrm{U} \\ 58.0 & 0 & 44 & 0.0046 & 1 & \mathrm{U} \\ 63.1 & 0 & 50 & 0.0018 & 1 & \\ 60.1 & 1 & 67 & 1.6 e-05 & 1 & \mathrm{U} \\ 61.2 & 1 & 24 & 0.62 & 1 & \\ 63.4 & 1 & 80 & 2.7 e-06 & 1 & \\ 62.7 & 1 & 50 & 0.0025 & 1 & \\ 59.6 & 0 & 85 & 9.4 e-07 & 1 & \mathrm{U} \\ 63.8 & 1 & 37 & 0.039 & 1 & \\ 61.7 & 0 & 89 & 3.7 e-07 & 1 & \mathrm{U}\end{array}$

Peptide

K. HIAEDADR . K

K. LDKENALDR . A

K. CAELEEELK.T + Carbamidomethyl (C)

K. LVIIESDLER. A

R. IQLVEEELDR . A

R. KLVIIESDLER. A

R. RIQLVEEELDR. A

K. KATDAEADVASLNR. R

K. ATDAFADVASLNRR. I

K. SIDDLEDELYAQK. L

R. IQLVEEELDRAQER . L

K. AISEELDHALNDMTSI .- 


\section{Protein View}

Match to: TMRRA score: 323

tropomyosin alpha chain, cardiac and skeletal muscle [validated] - rabbit

Found in search of ppw_K6_129990872610.txt

Nom1nal mass $\left(M_{r}\right)$ : 32661 ; Calculated pI value: 4.69

NCBI BLAST search of TMRBA aga1nst nr

Unformatted sequence string for pasting 1nto other applications

Taxonomy: oryctolagus cun1culus

L1nks to retr1eve other entr1es conta1n1ng this sequence from NCBI Entrez:

(no taxonomy 1nformation for this entry)

(no taxonomy 1 nformation for this entry)

(no taxonomy information for this entry)

(no taxonomy 1nformation for this entry)

(no taxonomy 1nformation for this entry)

(no taxonomy 1nformation for this entry)

(no taxonomy 1nformation for this entry)

(no taxonomy 1nformation for this entry)

(no taxonomy 1nformation for this entry)

(no taxonomy 1nformation for this entry)

Var1able mod1f1cat1ons: Carbam1domethy1 (C), Deam1dated (NQ), ox1dation (M)

Cleavage by Trypsin: cuts C-term side of KR unless next residue $1 \mathrm{~s} F$

sequence coverage: 33 s

Matched peptides shown in Bold Red

1 MDAIKKKMQM LKLDKENALD RAEQAEADKK AAEDRSKQLE DELVSLQKKL

51 KGTEDELDKY SEALKDAOEK LELAEKKATD AEADVASLNR RIQLVEEELD

101 RAOERLATAL OKLEEAEKAA DESERGMKVI ESRAOKDEEK MEIOEIOLKE

151 AKHIAEDADR KYEEVARKLV IIESDLERAE ERAELSEGKC AELEEELKTV

201 TNNLKSLEAQ AEKYSQKEDK YEEEIKVLSD KLKEAETRAE FAERSVTKLE

251 KSIDDLEDEL YAOKLKYKAI SEELDHALND MTSI

Show predicted peptides also

\section{Sort Peptides By}

- Residue Number

\section{Observed}

1073.6185

1460.8265

1488.8333

1399.8395

1243.7312

1728.0024

926.4818

1314.8416

1186.7365

1120.5782

1538.8136

\section{Mr (expt)}

1072.6112

1459.8192

1487.8260

1398,8322

1242.7239

1726.9951

925,4745

1313.8343

1185.7292

1119.5709

1537.8063

$269-284 \quad 1758.9299 \quad 1757.9226 \quad 1757.8141$

$269-284 \quad 1758.9299 \quad 1757.9226 \quad 1757.8141$

Increasing Mass

Decreasing Mass

Mr (calc)
1072.5513
1459.7267
1487.7328
1398.7467
1242.6456
1726.8849
925.4253
1313.7554
1185.6605
1119.5117
1537.7148
1757.8141

ppm
56
63
63
61
63
64
53
60
58
53
60
62

Miss Sequence

1 K. LDKENALDR.A (Ions score 49)

1 K. KATDAEADVASLNR. R (IOnS score 80)

1 K. ATDAFADVASLNRR. I (IOnS score 50)

1 R. RIQLVEEELDR. A (IONS score 24)

- R. IQLVEEELDR.A (IONS score 50)

1 R. IQLVEEELDRAQER. L (IONS SCOIE 37 )

0 K. HIAEDADR. $\mathrm{K}$ (IONS score 61)

1 R. KLVIIESDLER. A (IOnS Score 67)

0 K. LVIIESDIER.A (I OnS score 44)

0 K. CAELEEELK.T Carbam1domethy 1 (C)

0 K. SIDDLEDELYAOK, L

0 K. AISEELDHALNDMTSI.- (Ions score 89) 